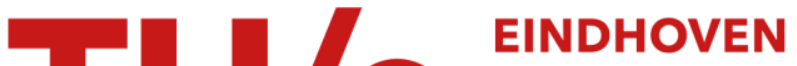 \\ UNIVERSITY OF \\ TECHNOLOGY
}

\section{Micromechanical modeling of the deformation kinetics of semicrystalline polymers}

\section{Citation for published version (APA):}

Sedighiamiri, A. (2012). Micromechanical modeling of the deformation kinetics of semicrystalline polymers. [Phd Thesis 1 (Research TU/e / Graduation TU/e), Mechanical Engineering]. Technische Universiteit Eindhoven. https://doi.org/10.6100//R735441

DOI:

10.6100/IR735441

Document status and date:

Published: 01/01/2012

\section{Document Version:}

Accepted manuscript including changes made at the peer-review stage

\section{Please check the document version of this publication:}

- A submitted manuscript is the version of the article upon submission and before peer-review. There can be important differences between the submitted version and the official published version of record. People interested in the research are advised to contact the author for the final version of the publication, or visit the $\mathrm{DOI}$ to the publisher's website.

- The final author version and the galley proof are versions of the publication after peer review.

- The final published version features the final layout of the paper including the volume, issue and page numbers.

Link to publication

\section{General rights}

Copyright and moral rights for the publications made accessible in the public portal are retained by the authors and/or other copyright owners and it is a condition of accessing publications that users recognise and abide by the legal requirements associated with these rights.

- Users may download and print one copy of any publication from the public portal for the purpose of private study or research.

- You may not further distribute the material or use it for any profit-making activity or commercial gain

- You may freely distribute the URL identifying the publication in the public portal.

If the publication is distributed under the terms of Article 25fa of the Dutch Copyright Act, indicated by the "Taverne" license above, please follow below link for the End User Agreement:

www.tue.nl/taverne

Take down policy

If you believe that this document breaches copyright please contact us at:

openaccess@tue.nl

providing details and we will investigate your claim. 
Micromechanical modeling of the deformation kinetics of semicrystalline polymers 


\section{CIP-DATA LIBRARY TECHNISCHE UNIVERSITEIT EINDHOVEN}

Micromechanical modeling of the deformation kinetics of semicrystalline polymers / by Amin Sedighiamiri. Technische Universiteit Eindhoven, 2012.

A catalogue record is available from the Eindhoven University of Technology Library ISBN: 978-90-386-3218-6

Subject headings: structure-property relation, micromechanical modeling, composite inclusion, plastic deformation, crystal plasticity, semicrystalline polymers, polyethylene, microstructure

Copyright (C) 2012 by Amin Sedighiamiri. All rights reserved.

Cover design: Paul Verspaget (Verspaget \& Bruinink)

Reproduction: University Press Facilities, Eindhoven, The Netherlands.

This research is part of the research programme of the Dutch Technology Foundation STW, "Predicting Catastrophic Failure of Semicrystalline Polymer Products". 


\section{Micromechanical modeling of the deformation kinetics of semicrystalline polymers}

\section{PROEFSCHRIFT}

ter verkrijging van de graad van doctor aan de Technische Universiteit Eindhoven, op gezag van de rector magnificus, prof.dr.ir. C.J. van Duijn, voor een commissie aangewezen door het College voor Promoties in het openbaar te verdedigen op maandag 17 september 2012 om 16.00 uur

door

Amin Sedighiamiri

geboren te Babol, Iran 
Dit proefschrift is goedgekeurd door de promotor:

prof.dr.ir. H.E.H. Meijer

\section{Copromotoren:}

dr.ir. J.A.W. van Dommelen

en

dr.ir. L.E. Govaert 


\section{Contents}

Summary v v v v v

1 Introduction 1

1.1 General introduction ............................................ 1

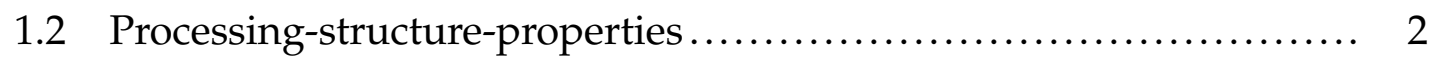

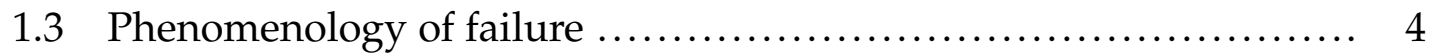

1.4 Deformation of semicrystalline polymers $\ldots \ldots \ldots \ldots \ldots \ldots \ldots \ldots \ldots \ldots$

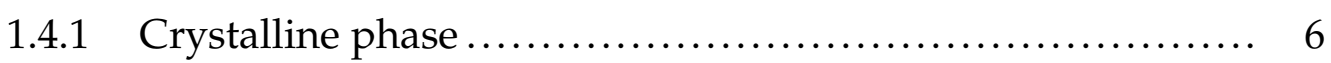

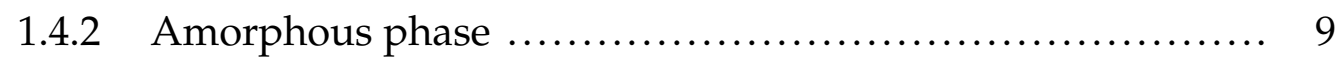

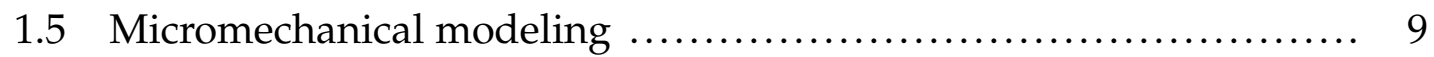

1.6 Outline of the thesis ........................................... 10

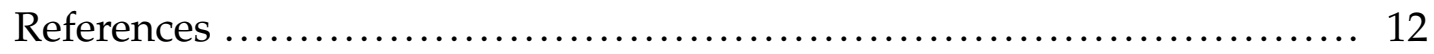

$\begin{array}{lll}2 & \text { Elastic properties } & 15\end{array}$

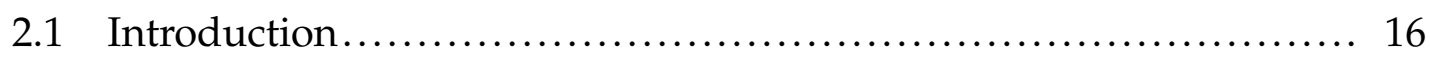

2.2 Experimental ...................................................... 18

2.2 .1 Sample preparation ........................................ 18

2.2.2 Crystallinity and lamellar thickness .......................... 19

$2.2 .3 \quad$ Mechanical testing ............................................. 19

2.3 Micromechanical modeling ....................................... 20

2.3 .1 Two-phase composite inclusion ............................ 21

2.3 .2 Towards a three-phase model ............................. 22

2.3 .3 Inclusion interaction law .................................... 25

2.4 Characterization of constituent phases ............................. 26 


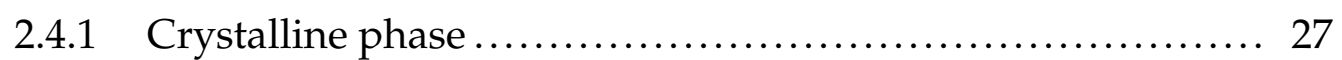

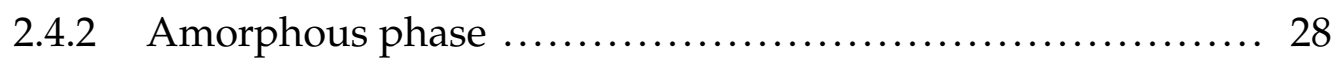

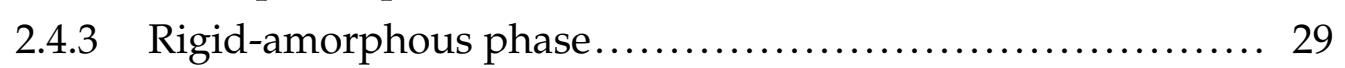

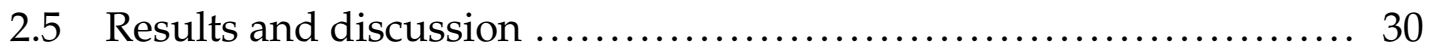

2.6 Conclusion .................................................. 34

2.A Appendix: Stiffness tensor of an elastic two-phase composite inclusion 36

2.B Appendix: Stiffness tensor of an elastic three-phase composite inclusion 39

2.C Appendix: $\hat{e}$-inclusion model ................................... 42

References ........................................................... 44

3 Deformation kinetics and texture evolution $\quad 47$

3.1 Introduction ...................................................... 48

3.2 Experimental ..................................................... 50

3.3 Model description.................................................. 50

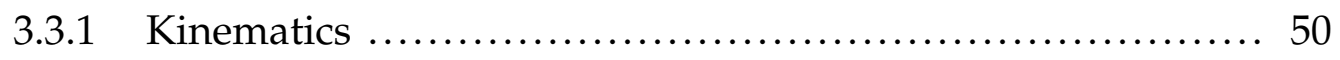

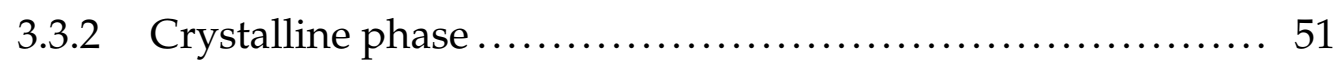

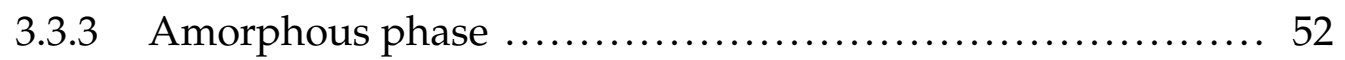

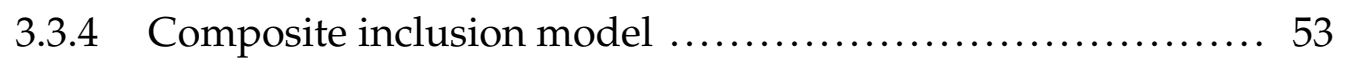

3.3 .5 Texture evolution ........................................ 54

3.3 .6 Deformation modes ........................................... 54

$3.4 \quad$ Initial model parameters and results ................................. 55

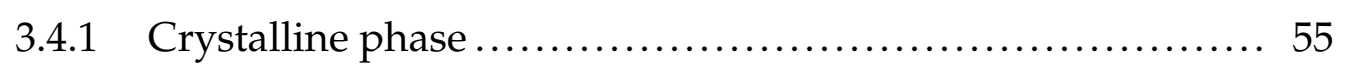

3.4 .2 Amorphous phase ........................................ 56

3.4.3 Initial crystalline and lamellar orientations ................. 57

3.4.4 Application: uniaxial tension ............................ 58

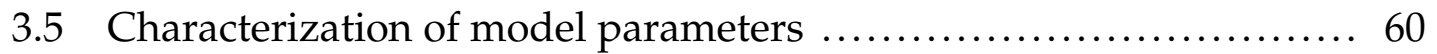

3.6 Discussions and conclusions ......................................... 68

3.A Appendix: Micromechanical model ................................ 71

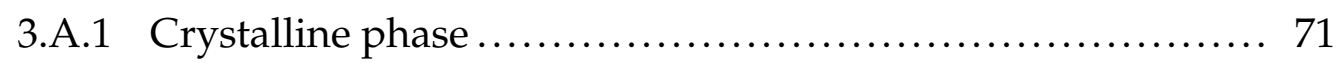

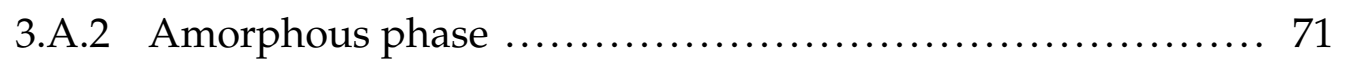

3.A.3 Composite inclusion ........................................ 72

3.A.4 Hybrid interaction law ....................................... 73

References ........................................................ 75 
4 Short- and long-term failure kinetics $\quad 79$

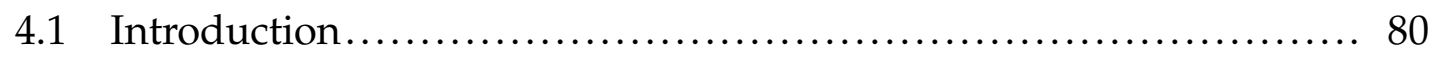

4.2 Experimental ............................................ 83

$4.2 .1 \quad$ Material ........................................... 83

4.2 .2 Sample preparation ................................. 83

4.2 .3 Mechanical testing ..................................... 83

4.2.4 Size exclusion chromatography (SEC) ..................... 83

4.3 Constitutive modeling ......................................... 84

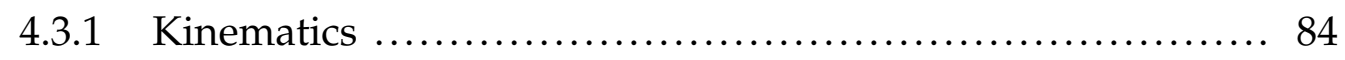

4.3 .2 Crystalline domain.................................. 84

4.3 .3 Amorphous domain ................................ 86

4.3 .4 Composite inclusion model .......................... 87

4.3.5 Deformation modes .................................... 88

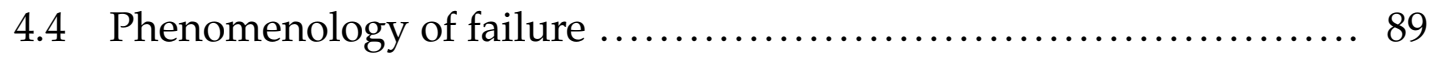

4.5 Results and discussion ....................................... 91

4.6 Conclusions .................................................. 103

4.A Appendix: Multi-scale constitutive model ........................ 105

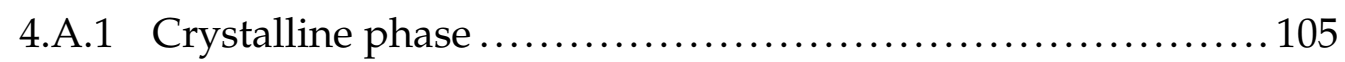

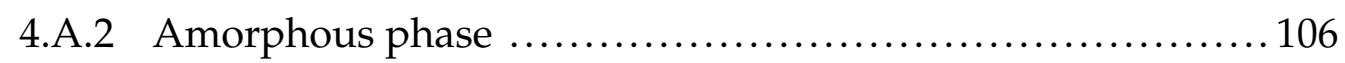

4.A.3 Composite inclusion model ............................. 107

4.A.4 Hybrid interaction law ................................... 107

4.B Appendix: Viscosity expression ................................ 109

References .................................................... 111

5 Deformation kinetics of oriented polymers 117

5.1 Introduction ...................................................... 118

5.2 Experimental .............................................. 120

5.2 .1 Material ..................................................... 120

5.2 .2 Crystallinity ........................................ 120

5.2 .3 Orientation measurement................................. 121

$5.2 .4 \quad$ Mechanical testing .................................. 123

5.3 Model description ............................................ 124 


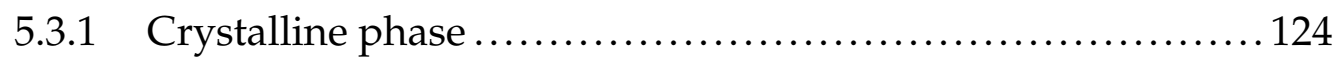

$5.3 .2 \quad$ Amorphous phase ........................................ 125

5.3.3 Composite inclusion model .................................. 126

5.3.4 Initial orientations........................................... 126

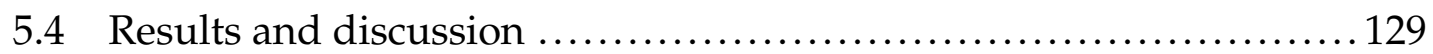

5.4 .1 Isotropic amorphous domains ............................. 130

5.4 .2 Oriented amorphous domains ............................. 131

5.4.3 Probing characterization of crystallographic slip systems ..... 136

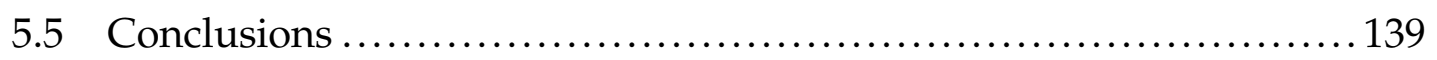

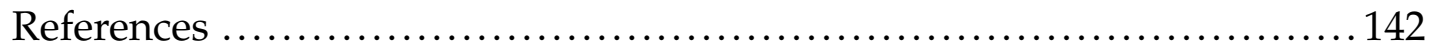

6 Conclusions and recommendations $\quad 147$

6.1 Conclusions ..................................................... 147

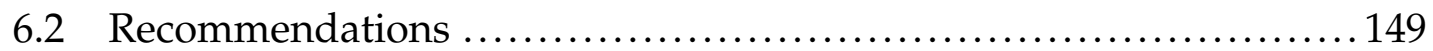

References ............................................................ 153

\begin{tabular}{ll}
\hline Samenvatting & 155
\end{tabular}

\begin{tabular}{ll}
\hline acknowledgements & 157
\end{tabular}

$\begin{array}{ll}\text { Curriculum Vitae } & 159\end{array}$ 


\section{Summary}

The process of plastic deformation in semicrystalline polymers is complicated due to the operation of a variety of mechanisms at different levels and is strongly dependent on their crystallinity level, the initial underlying microstructure, and the evolution of this structure during deformation. Any macroscopically homogeneous deformation is accommodated by various deformation mechanisms in the heterogeneous microstructure. The objective of this work is to establish a quantitative relation between the microstructure and the mechanical performance of semicrystalline polymers, as characterized by elasto-viscoplastic deformation. In order to do that, a micromechanically based constitutive model is used. The model represents the microstructure as an aggregate of layered composite inclusions, each consisting of a crystalline lamella, which is mechanically coupled to its adjacent amorphous layer. The crystalline phase is modeled as anisotropic elastic with plastic flow governed by crystallographic slip. The amorphous phase is assumed to be isotropic elastic with a rate dependent plastic flow and strain hardening resulting from molecular orientation. To relate the volume-averaged mechanical behavior of each layered composite inclusion to the aggregate of composite inclusions, a hybrid local-global interaction law is used.

The concept of a layered composite inclusion as a representative element is extended with a third phase, which is also referred to as the interphase or the rigid-amorphous phase. This phase represents the region between crystalline and amorphous domains, having a somewhat ordered structure and a fixed thickness. The incorporation of the interphase in the composite inclusion model naturally leads to a dependence on the lamellar thickness, i.e. on an internal length scale. This rigid-amorphous phase is particularly relevant for quantitative modeling of the behavior of oriented semicrystalline structures. A comparison with experimental data shows a good prediction with the two-phase model for isotropic material.

A critical factor for adding quantitative predictive abilities to the micromechanical model for prediction of the elasto-viscoplastic behavior in semicrystalline polymers is the stress-dependence of the rate of plastic deformation, the slip kinetics, which is the mechanism underlying time-dependent, macroscopic failure. 
The kinetics of the macroscopic plastic flow strongly depend on the slip kinetics of the individual crystallographic slip systems, accompanied by the yield kinetics of the amorphous domain. To obtain an accurate quantitative prediction, the viscoplastic power law relation, normally used in micromechanical modeling, is replaced with an Eyring flow rule. The slip kinetics are then re-evaluated and characterized using a hybrid numerical/experimental procedure, and the results are validated for uniaxial compression data of HDPE. A double yield phenomenon is observed in the model prediction, and is found to be related to morphological changes during deformation, which induce a change of deformation mechanism.

Experimental data on the yield kinetics of polyethylene at different temperatures and strain rates reveals the contribution of two relaxation processes. Further experimental observations on the stress dependence of the time-to-failure show a piecewise linear relation in semi-logarithmic plots, with the same slope as that of the yield kinetics. This indicates that the kinetics of failure under applied strain-rate and applied stress are strongly related. To predict failure under both conditions and for different temperatures, the crystallographic slip kinetics and the amorphous yield kinetics were further refined, and the Eyring flow rule was modified by adding a temperature shift function. The creep behavior of polyethylene was then simulated directly using the multi-scale, micromechanical model, predicting the time-to-failure without any additional fitting parameter. To enable the prediction of both tension and compression, a non-Schmid effect is added to the constitutive relation of each slip system.

Injection molded or extruded polymers possess a different morphology than isotropic polymers, due to the subjection to shear and elongational flow during processing. Therefore, their plastic deformation and failure behavior are anisotropic. The relation between the initially oriented microstructure and the deformation kinetics of oriented polyethylene tapes is investigated using the multi-scale micromechanical model. The initial orientation distribution for the model is obtained based on wide angle X-ray scattering experiments. Due to the presence of oriented amorphous domains in the drawn samples, the macroscopic plastic flow is predominantly governed by the yield kinetics of the amorphous phase. The necessity of modeling the load angle dependence of the properties of the oriented amorphous domain for an accurate quantitative prediction is discussed. Furthermore, the possibilities for identifying the properties of distinct crystallographic slip systems are investigated. 


\title{
Introduction
}

\section{Chapter 1}

\begin{abstract}
A general introduction to the relation between structure and mechanical properties of semicrystalline polymers is presented, as well as the underlying deformation mechanisms. An overview of the micromechanically based approach used for the modeling of the mechanical behavior of these materials, involving different length scales, is given.
\end{abstract}

\subsection{General introduction}

Due to many advantages such as low cost and weight, polymeric materials are increasingly integrated in everyday life and are being used in a wide range of applications, like packaging, building and construction, medical, household, electrical, automotive, agriculture, etc. With an average annual growth rate of $8.1 \%$, the world consumption of solid polymers increased from 7 million tons in 1960 to 196 million tons in 2005. Using a more conservative annual growth rate of $6.5 \%$, world polymer consumption will be 540 million tons in year 2020 [1].

Depending on the arrangement of molecular chains, polymers can be amorphous with molecules lacking any positional order, or semicrystalline containing both ordered crystalline and randomly coiled amorphous regions. One of the most important advantages of semi-crystalline polymers is that their maximum temperature of use is set by the melting temperature $T_{m}$, whereas the maximum use temperature of amorphous polymers is set by the glass transition temperature $T_{g}$, always lower than $T_{m}$. Of the world's plastic consumption in year 2005, 68 million tons consisted of polyethylene [2], being one of the most important semicrystalline polymers with many daily life and engineering applications. 


\subsection{Processing-structure-properties}

The mechanical performance of a semicrystalline polymeric product is strongly dependent on the microstructural features, such as the degree of crystallinity, crystal size and initial orientation, which themselves are determined by the thermo-mechanical history experienced by the polymer during processing of the product [3-6]. Figure 1.1 lists four basic microstructures, often observed in semicrystalline polymers. When slowly cooled from the melt in the absence of

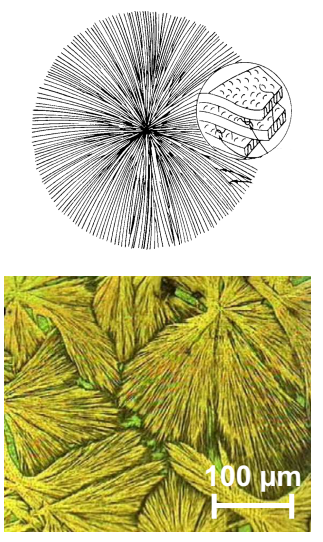

a
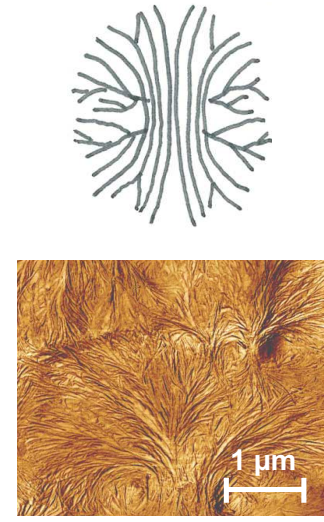

b
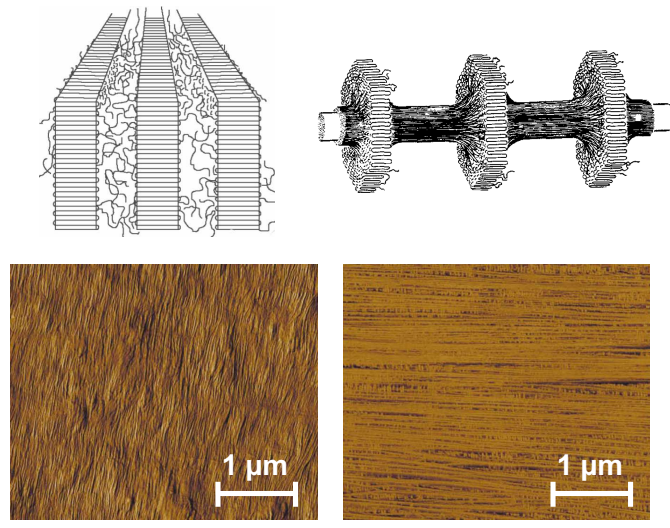

C

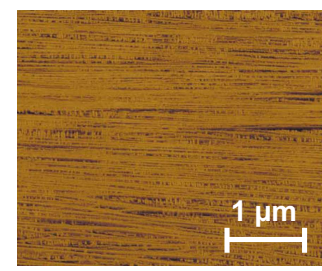

d

flow

Figure 1.1 Morphologies, often observed in semicrystalline polymers: (a) Optical image of isotropic spherulites, reproduced from [7] and the schematic representation from [8]. (b) A schematics representation and an AFM image of partially oriented spherulites, reproduced from [9]. (c) A schematics representation and an AFM image of a rowstructure, reproduced from [9]. (d) An AFM image of a shish-kebab structure, reproduced from [9] and a schematic representation from [10].

flow, so-called quiescent crystallization, semicrystalline polymers often show a spherulitic morphology [8, 11, 12]. Each spherulite consists of a radial assembly of thin crystalline lamellae which are separated by amorphous layers, figure 1.1a. Under conditions of relatively weak flow of the polymer during processing, nucleation of spherulitic structures may be significantly enhanced [13, 14], and slightly oriented spherulitic structures are formed, as can be seen in figure 1.1b. At increasing flow rates or times, the crystalline morphology changes and oriented crystalline structures such as stacked lamellae (row-structure) and a shish-kebab-like morphology are observed [9, 15, 16], as shown in figure 1.1k 
and $1.1 \mathrm{~d}$, respectively. In injection molding of semicrystalline polymers, during which the flow rate often shows strong variations throughout the mold, in general, all these basic structures may be found [5, 6]. For example, the absence of shear in the center of an injection-molded product results in a spherulitic structure, while in the regions at the cavity walls that experience a strong flow, an oriented structure can be present.

An illustrative example of the influence of different structures, formed during processing, on the mechanical performance of semicrystalline polymers is given in figure 1.2, which shows an injection-molded plate of high-density polyethylene (HDPE), revealing a homogeneous core and oriented layers of different thickness at various locations with respect to the die along the flow direction [6]. The differ-
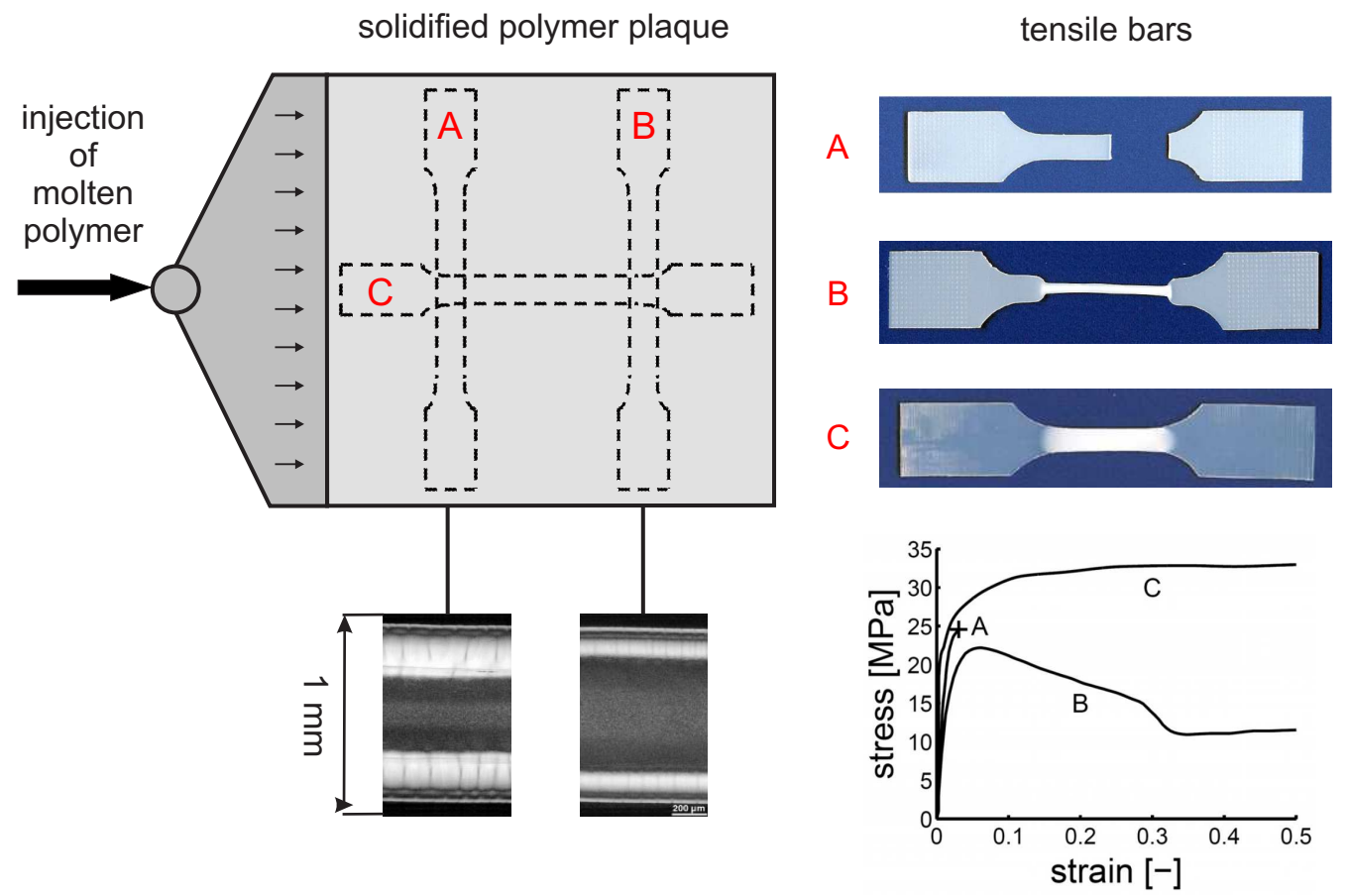

Figure 1.2 Variation of microstructure over the thickness in injection-molding of HDPE (left), and the resulting different mechanical responses of samples A, B and C, cut from different parts of the injection-molded plate (right).

ences observed in the microstructure have a drastic influence on the macroscopic mechanical response of specimens cut at different locations and orientations from the injection-molded plate, which range from brittle fracture, to neck formation and homogeneous deformation, samples A, B and C, respectively. From this simple experiment, it is evident that the mechanical properties of polymer products are strongly linked to their underlying microstructure. Therefore, 
the objective of this work is to establish a quantitative relation between the microstructure and the mechanical performance of semicrystalline polymers, as characterized by elasto-viscoplastic deformation.

\subsection{Phenomenology of failure}

In order to predict the mechanical behavior of semicrystalline polymers, one first needs to further investigate their yield and failure kinetics under both shortterm and long-term mechanical loadings. Figure 1.3 a illustrates the short-term mechanical behavior of HDPE, measured in a tensile test with a constant strain rate. The material displays an initially elastic region, where the stress increases linearly with strain. At higher stresses, the response becomes nonlinear and eventually reaches a maximum, the so-called engineering yield stress. Soon after, the material experiences necking due to strain localization [17] and a localized plastic zone is formed, which can propagate along the entire length of the test bar. From a mechanical point of view, the moment of neck initiation is regarded as the point of failure, i.e. where the material loses its integrity.

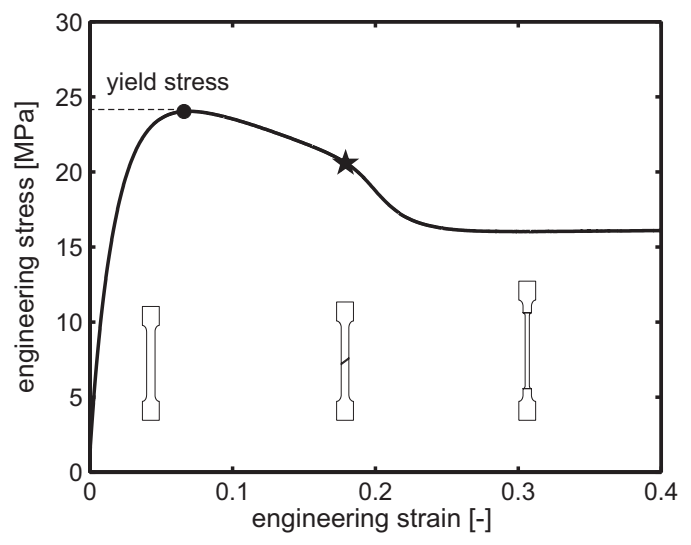

a

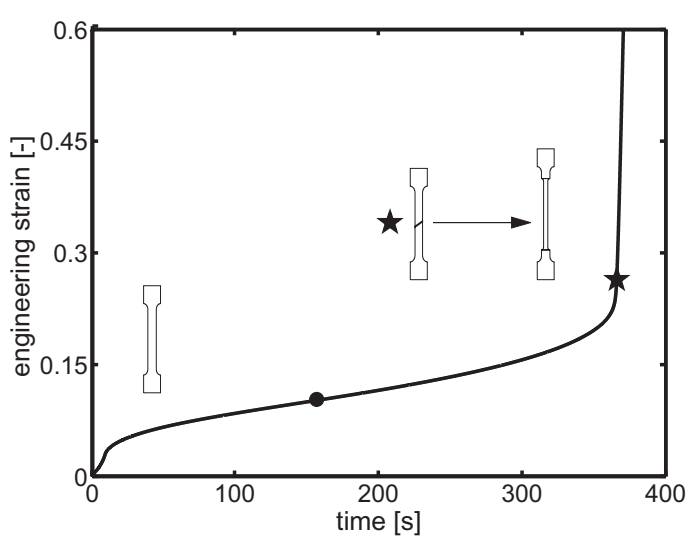

b

Figure 1.3 (a) Deformation behavior of isotropic HDPE in uniaxial tension under a constant strain rate of $0.001 \mathrm{~s}^{-1}$. (b) Creep behavior of the same material under a constant applied stress of $20 \mathrm{MPa}$.

Figure 1.3b shows the long-term performance of the material, in which the evolution of the engineering strain under a constant applied stress that is approximately $16 \%$ below the yield stress, is illustrated. As can bee seen, the deformation increases slowly in time, with a rate of deformation becoming constant (flow regime) after an initial viscoelastic region (primary creep). However, the rate of deformation abruptly increases at longer loading times, resulting 
in the failure of the material. The observed mode of failure is similar to that observed in short-term performance, i.e. necking. The key issue, here, is that the applied stress dependency of the steady-state creep rate at the flow regime is interchangeable with the strain rate dependency of the yield stress.

The time scale at which a polymer material fails, depends on the loading condition applied. This is illustrated in figure 1.4, which shows the deformation of an isotropic HDPE in uniaxial tension under different constant strain rates, and the creep response of the same material under different constant applied stresses. It can be concluded from this figure that for polymer materials, due to their timedependent mechanical response, it is not the question whether the material fails under static loading, but rather when the material fails under a designed load specification. The higher the strain rate, the higher the yield stress, and the higher the level of the applied load, the lower the time-to-failure. The short-term and

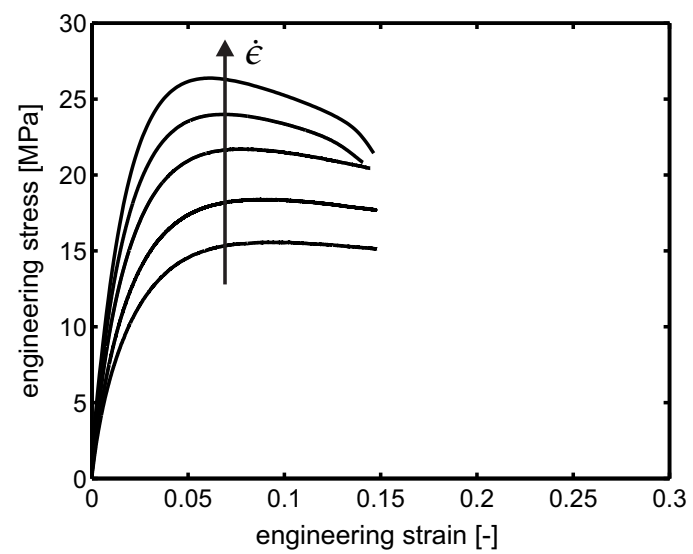

a

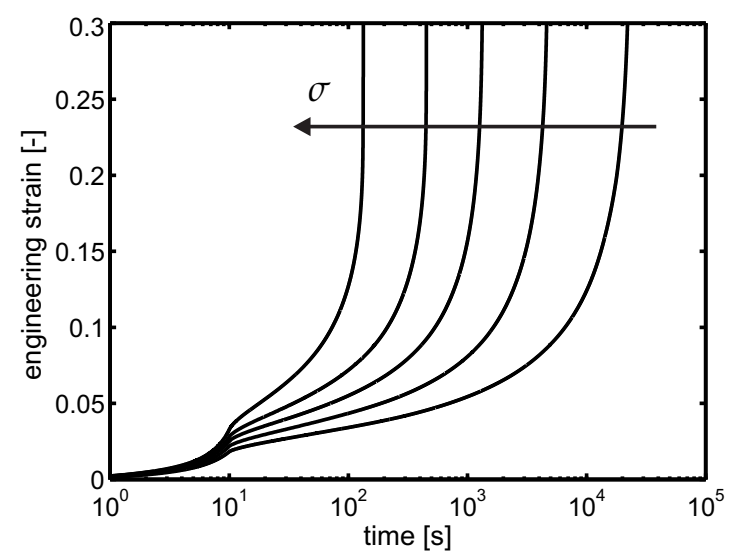

b

Figure 1.4 (a) Tensile behavior of isotropic HDPE under different constant strain rates. (b) Creep behavior of the same material under different constant applied stresses.

long-term kinetics of isotropic HDPE at the ambient temperature are given in figure 1.5. As can be seen, both strain rate dependence of the yield stress and the applied stress dependence of the time-to-failure give a linear relation in a semilogarithmic scale, with a same absolute slope $\alpha$. The observation supports the idea that the kinetics of failure under applied strain rate and applied stress are strongly related.

\subsection{Deformation of semicrystalline polymers}

Semicrystalline polymers possess a crystalline phase which consists of regularly ordered chain segments, and amorphous domains in which molecules are ar- 


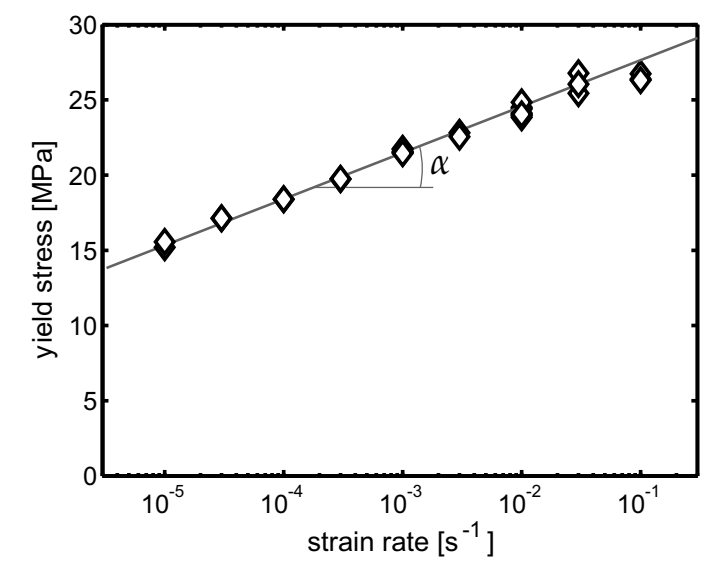

a

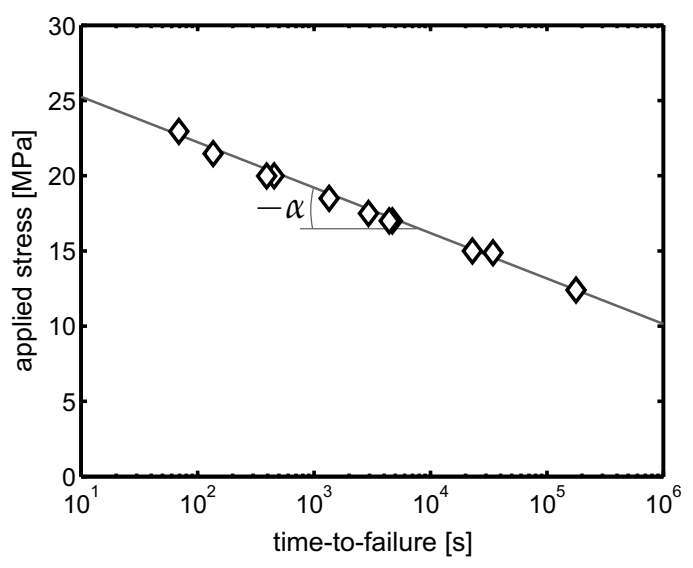

b

Figure 1.5 (a) Strain rate dependence of the yield stress and (b) applied stress dependence of time-to-failure for isotropic $\mathrm{HDPE}$ at $25^{\circ} \mathrm{C}$.

ranged with a randomly coiled character, and which are constrained between neighboring crystalline lamellae. These two constituent phases form a lamellar morphology, characterized by crystallographic texture (crystalline lattice orientation) and morphological texture (crystalline-amorphous interface orientation). The plastic deformation of semicrystalline polymers is complicated due to the operation of a variety of mechanisms at different levels and is strongly dependent on the percentage crystallinity, the initial crystallographic and morphological textures, as well as the evolution of these textures with ongoing deformation. Any macroscopically homogeneous deformation is accommodated by various deformation mechanisms in the heterogeneous microstructure [18-22]. High density polyethylene (HDPE) will be used as an example throughout this thesis.

\subsubsection{Crystalline phase}

The most common crystalline structure for polyethylene is the orthorhombic unit cell [23]. The microstructural mechanisms that control the plastic deformation of the crystalline phase in semicrystalline polymers are assigned primarily to the crystallographic slip process up to moderate strains [18, 20, 21]. However, polymer crystals may also deform plastically by mechanical twinning and stressinduced martensitic phase transformation [19, 21]. Crystallographic slip is the main deformation mode up to moderate strains, and occurs by gliding of macromolecular chains along each other on crystallographic slip planes within the crystalline lamella. When the direction of slip is parallel to the chain axis, the deformation mechanism is referred to as chain slip, whereas when the slip 
direction is perpendicular to the chain direction, the slip mechanism is called transverse slip, as schematically shown in figure 1.6.

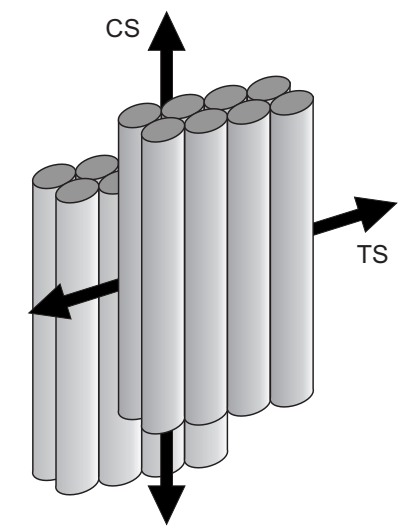

Figure 1.6 Schematic representation of chain slip (CS) and transverse slip (TS) in polymer crystals.

During the early stages of deformation, plastic deformation predominantly occurs by fine slip [8, 18], where a small amount of deformation is equally distributed on a large number of slip planes. At larger strains, fine slip is accompanied by a process of coarse slip, with large deformations on a few slip planes, resulting in the break-up of crystalline lamellae [24, 25]. These two slip mechanisms are schematically illustrated in figure 1.7. As can be seen, the fine

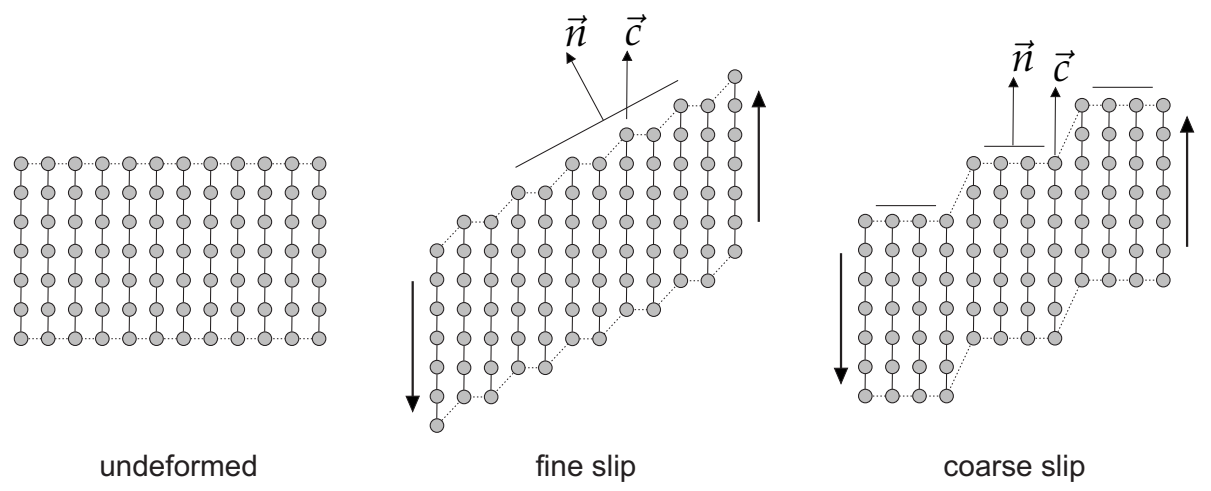

Figure 1.7 Schematic illustration of fine and coarse chain slip mechanisms, after [26].

slip process changes the angle between the chain direction and the interface normal, whereas coarse slip does not alter the chain tilt angle.

The crystalline phase of polyethylene employs eight physically distinct slip systems [20], which comprise six crystallographically different types of slip 
systems, each possibly having different properties. Figure 1.8 shows all slip systems present in HDPE. This set of slip systems effectively comprises four linearly independent deformation modes.
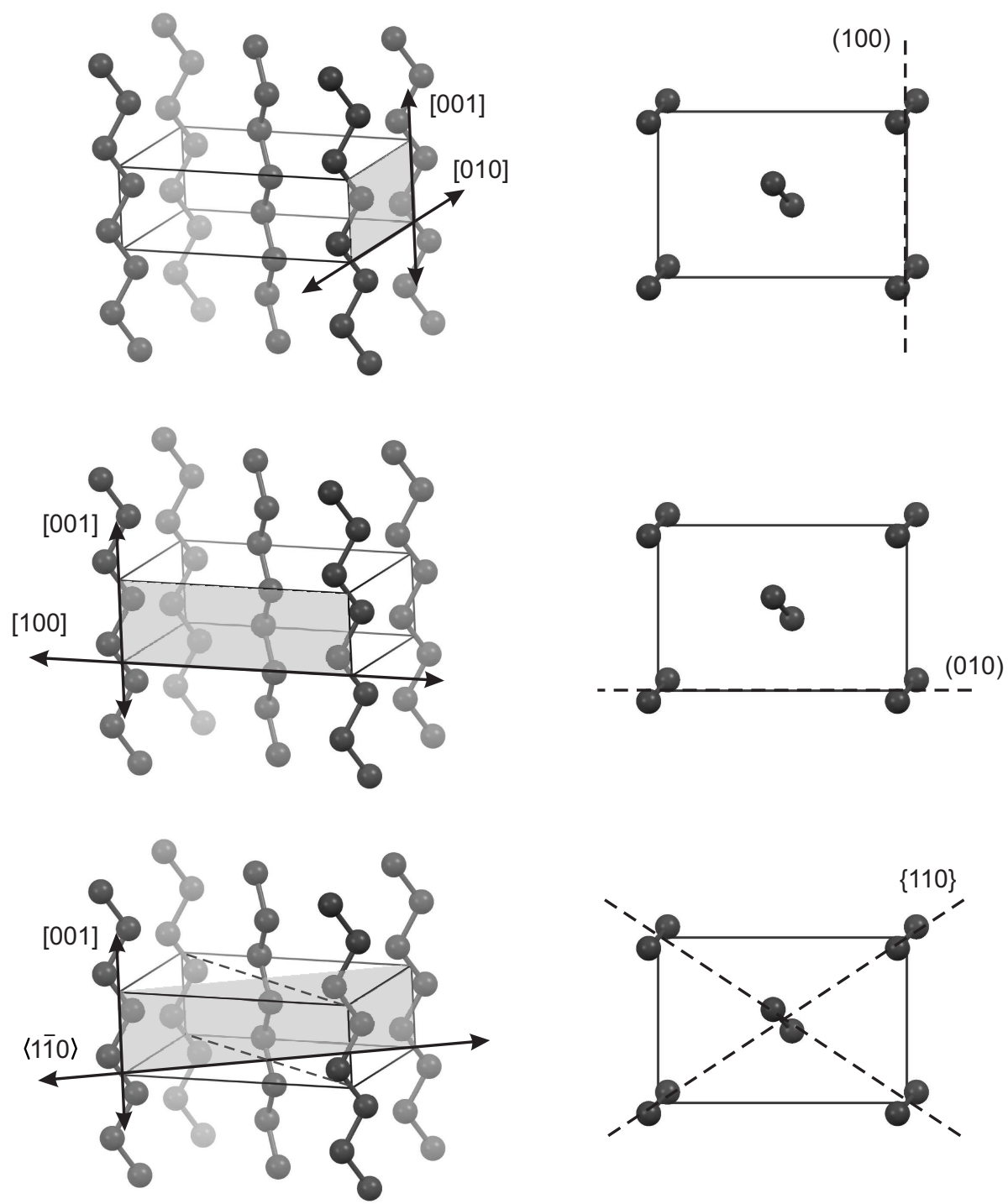

Figure 1.8 Schematic illustration of all physically distinct slip systems in the crystalline domain of HDPE. 


\subsubsection{Amorphous phase}

The amorphous phase of semicrystalline polyethylene consists of an assembly of randomly coiled macromolecules, strongly connected to their neighboring crystalline lamellae through tie molecules, loose chain folds, cilia and intercrystalline chains crossing the interface of the crystalline and amorphous phase. Due to the strong connectivity of the constituent phases in semicrystalline polymers through many chains crossing the crystalline/amorphous interface, the crystallographic mechanisms are inevitably accompanied by interlamellar deformation and they can only deform simultaneously [22]. The deformation of the amorphous phase can lead to either interlamellar shear or interlamellar separation.

\subsection{Micromechanical modeling}

In order to establish a quantitative relation between the microstructure and the mechanical performance of semicrystalline polymers, as characterized by elastoviscoplastic deformation, a micromechanically based constitutive model [27-29] is used. Two different length scales are distinguished in the employed multiscale, micromechanical model, namely the microscopic and macroscopic scale, as is schematically depicted in figure 1.9. At the microscopic scale, a twophase layered composite entity [27, 28] is used as a representative microstructural element, based on the lamellar structure that is commonly observed in semicrystalline polymers. Each separate composite entity consists of a crystalline lamella, mechanically coupled to its corresponding amorphous layer to form a lamellar morphology. The yield kinetics are defined at the level of the crystallographic slip systems and the amorphous phase. A key issue is the stress-dependence of the microscopic rate of plastic deformation, the slip kinetics, which is the mechanism underlying time-dependent, macroscopic failure. The kinetics of the macroscopic plastic flow strongly depends on the rate-dependence of slip along crystallographic planes, together with the yield kinetics of the amorphous domain. Therefore, an accurate quantitative prediction requires a proper description of the slip kinetics and amorphous yield kinetics.

At the macroscopic level, the material is represented by an aggregate of a discrete number of composite inclusions. The mechanical response of the aggregate to the boundary conditions imposed, is then computed by relating the volumeaveraged deformation and stress fields of each layered composite inclusion to the corresponding fields of the aggregate using a hybrid local-global interaction law [27-29]. 


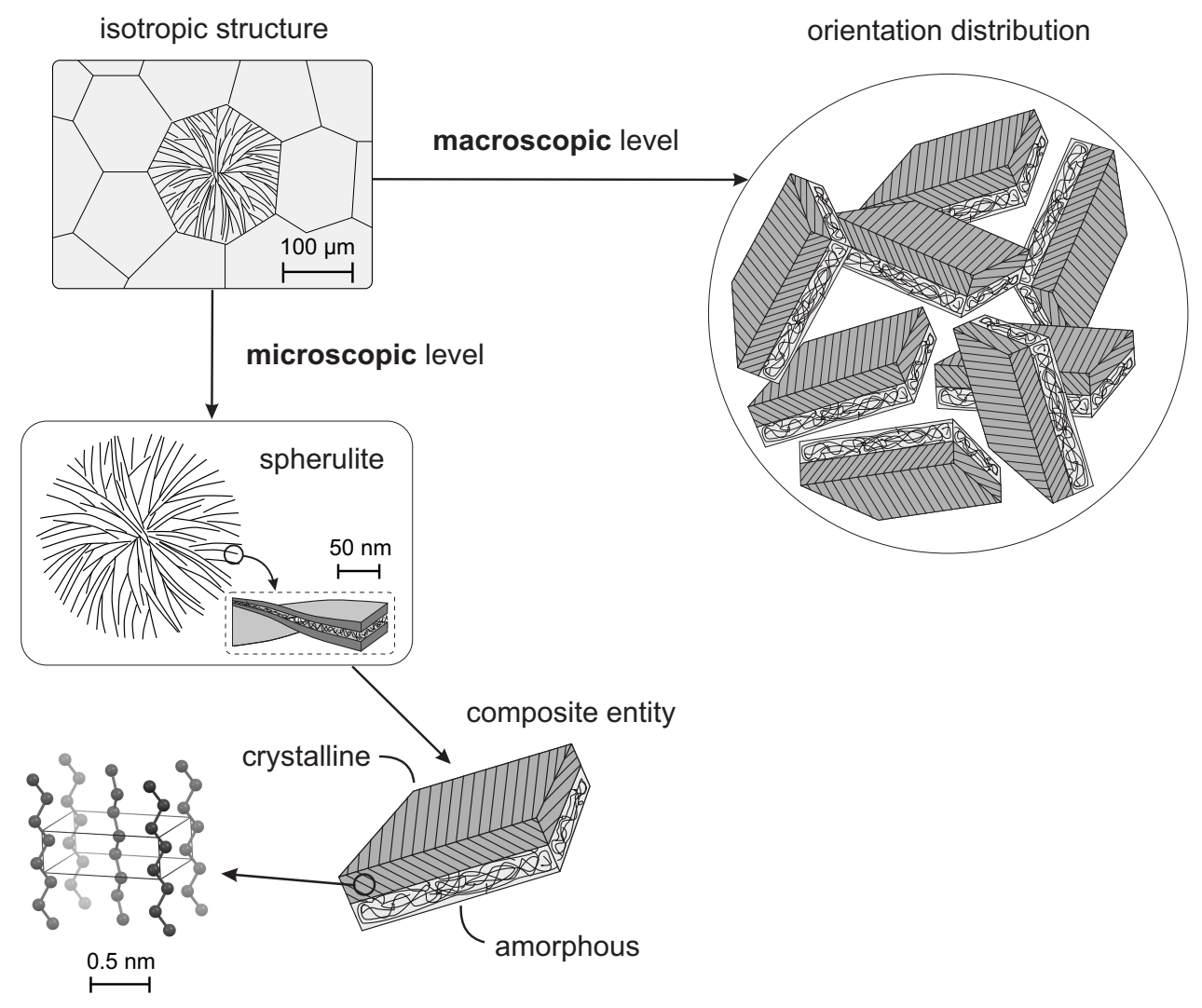

Figure 1.9 Schematic representation of the microscopic and macroscopic scales, included in the multi-scale, micromechanical model.

\subsection{Outline of the thesis}

In chapter 2, the concept of a layered composite inclusion as a representative element is extended in an elastic framework by incorporating a third phase, which is referred to as the interphase or the rigid-amorphous phase, having a rather ordered structure and a constant thickness. The three-phase model incorporates an internal length scale through crystalline lamellar and interphase thicknesses, whereas no length scales are included in the two-phase model. A linear elastic composite inclusion model is used to obtain a closed form solution for the stiffness tensor of the semicrystalline polymer. The model results are compared with experimental data to assess the capabilities of the two- or threephase composite inclusion model.

Chapter 3 is mainly directed towards adding quantitative predictive abilities to the multi-scale, micromechanical model to capture the elasto-viscoplastic deformation and texture evolution in semicrystalline polymers at room tem- 
perature. As a first step in obtaining an accurate quantitative prediction, the viscoplastic power law relation is replaced with an Eyring flow rule. The slip kinetics and the amorphous yield kinetics are then characterized using a hybrid numerical/experimental procedure, and the results are validated for uniaxial compression data of HDPE.

Experimental observations on both the yield kinetics and the time-to-failure of polyethylene at different temperatures reveal the contribution of two relaxation processes. Chapter 4 focuses on the prediction of this thermo-rheologically complex short-term and long-term failure of polyethylene, which requires further re-evaluation of the crystallographic slip kinetics and the amorphous yield kinetics. To predict failure at different temperatures, the general Eyring flow rule was modified by adding a temperature shift function. A non-Schmid effect is added to the constitutive relation of each slip system to enable the prediction of both tension and compression.

The deformation kinetics of oriented semicrystalline polymers is discussed in chapter 5. Due to the effect of shear and elongational flow during processing, injection molded or extruded polymers possess a different structure than isotropic polymers, and their plastic deformation and failure are anisotropic. The predictive ability of the micromechanical model, together with the characterization of slip kinetics, is evaluated for oriented systems. The initial orientation distribution of the material is obtained from WAXS experiments.

Finally, conclusions and some recommendations are given in chapter 6 . 


\section{References}

[1] F. Pardos. Plastics in the Twenties. In: ANTEC 2004 Plastics: Annual Technical Conference, Volume 3: Special Areas. Chicago, 2004.

[2] F. Pardos. Market overview: Multilayer films. In: International conference of multilayer films. Brussels, 2005.

[3] M.R. Kantz, H.D. Newman, and Stigale F.H. The skin core morphology and structureproperty relationships in injection-molded polypropylene. Journal of Applied Polymer Science 16:1249-1260, 1972.

[4] J.P. Trotignon and J. Verdu. Skin-core structure-fatigue behavior relationships for injectionmolded parts of polypropylene. I. Influence of molecular weight and injection conditions on the morphology. Journal of Applied Polymer Science 34:1-18, 1987.

[5] C.M. Hsiung and M. Cakmak. Effect of processing conditions on the structural gradients developed in injection-molded poly(aryl ether ketone) (PAEK) parts. I. Characterization by microbeam X-ray diffraction technique. Journal of Applied Polymer Science 47:125-147, 1993.

[6] B.A.G. Schrauwen, L.C.A. Van Breemen, Spoelstra A.B., Govaert L.E., Peters G.W.M., and Meijer H.E.H. Structure, deformation, and failure of flow-oriented semicrystalline polymers. Macromolecules 37:8618-8633, 2004.

[7] N. Sidelman, Y. Rosenberg, and S. Richter. Peptide-based spherulitic films formation and properties. Journal of Colloid and Interface Science 343:387-391, 2010.

[8] L. Lin and A.S. Argon. Structure and plastic deformation of polyethylene. Journal of Materials Science 29:294-323, 1994.

[9] J. Xu, M. Johnson, and G.L. Wilkes. A tubular film extrusion of poly(vinylidene fluoride): structure/process/property behavior as a function of molecular weight. Polymer 45:53275340, 2004.

[10] P.J. Barham and A. Keller. High-strength polyethylene fibres from solution and gel spinning. Journal of Materials Science 20:2281-2302, 1985.

[11] D.C. Bassett and A.M. Hodge. On the morphology of melt-crystallized polyethylene I. Lamellar profiles. Proceedings of the Royal Society of London A 377:25-37, 1981.

[12] D.C. Bassett, A.M. Hodge, and R.H. Olley. On the Morphology of Melt-Crystallized Polyethylene II. Lamellae and their Crystallization Conditions. Proceedings of the Royal Society of London A 377:39-60, 1981.

[13] C. Tribout, B. Monasse, and J.M. Haudin. Experimental study of shear-induced crystallization of an impact polypropylene copolymer. Colloid and Polymer Science 274:197$208,1996$.

[14] H. Janeschitz-Kriegl, E. Ratajski, and M. Stadlbauer. Flow as an effective promotor of nucleation in polymer melts: a quantitative evaluation. Rheologica acta 42:355-364, 2003.

[15] G. Eder and H. Janeschitz-Kriegl. Structure development during processing: Crystallization. In: Materials Science and Technology. Wiley-VCH Verlag GmbH, 1997.

[16] R.H. Somani, L. Yang, B.S. Hsiao, P.K. Agarwal, H.A. Fruitwala, and A.H. Tsou. Shearinduced precursor structures in isotactic polypropylene melt by in-situ rheo-SAXS and rheo-WAXD studies. Macromolecules 35:9096-9104, 2002. 
[17] P.I. Vincent. The necking and cold-drawing of rigid plastics. Polymer 1:7-19, 1960.

[18] P.B. Bowden and R.J. Young. Deformation mechanisms in crystalline polymers. Journal of Materials Science 9:2034-2051, 1974.

[19] R.J. Young and P.B. Bowden. Twinning and martensitic transformations in oriented highdensity polyethylene. Philosophical Magazine 29:1061-1073, 1974.

[20] Z. Bartczak, A.S. Argon, and R.E. Cohen. Deformation mechanisms and plastic resistance in single-crystal-textured high-density polyethylene. Macromolecules 25:5036-5053, 1992.

[21] A. Galeski. Strength and toughness of crystalline polymer systems. Progress in Polymer Science 28:1643-1699, 2003.

[22] Z. Bartczak and A. Galeski. Plasticity of Semicrystalline Polymers. Macromolecular Symposia 294:67-90, 2010.

[23] C. G'sell and A. Dahoun. Evolution of microstructure in semi-crystalline polymers under large plastic deformation. Materials Science and Engineering: A 175:183-199, 1994.

[24] M.F. Butler, A.M. Donald, and A.J. Ryan. Time resolved simultaneous small- and wideangle X-ray scattering during polyethylene deformation: 1. Cold drawing of ethylene- $\alpha-$ olefin copolymers. Polymer 38:5521-5538, 1997.

[25] V. Gaucher-Miri and R. Séguéla. Tensile yield of polyethylene and related copolymers: mechanical and structural evidences of two thermally activated processes. Macromolecules 30:1158-1167, 1997.

[26] R.J. Young. Screw dislocation model for yield in polyethylene. Materials forum 11:210-216, 1988.

[27] B.J. Lee, D.M. Parks, and S. Ahzi. Micromechanical modeling of large plastic deformation and texture evolution in semicrystalline polymers. Journal of the Mechanics and Physics of Solids 41:1651-1687, 1993.

[28] B.J. Lee, A.S. Argon, D.M. Parks, S. Ahzi, and Z. Bartczak. Simulation of large strain plastic deformation and texture evolution in high density polyethylene. Polymer 34:3555-3575, 1993.

[29] J.A.W. van Dommelen, D.M. Parks, M.C. Boyce, W.A.M Brekelmans, and F.P.T. Baaijens. Micromechanical modeling of the elasto-viscoplastic behavior of semi-crystalline polymers. Journal of the Mechanics and Physics of Solids 51:519-541, 2003. 



\title{
Elastic properties
}

Chapter 2

\begin{abstract}
The mechanical performance of semicrystalline polymers is strongly dependent on their underlying microstructure, consisting of crystallographic lamellae and amorphous layers. In line with that, semicrystalline polymers have previously been modeled as two and three-phase composites, consisting of a crystalline and amorphous phase and, in case of the threephase composite, a rigid-amorphous phase between the other two, having a somewhat ordered structure and a constant thickness. In this work, the ability of two-phase and three-phase composite models to predict the elastic modulus of semicrystalline polymers is investigated. The three-phase model incorporates an internal length scale through crystalline lamellar and interphase thicknesses, whereas no length scales are included in the twophase model. Using linear elastic behavior for the constituent phases, a closed form solution for the average stiffness of the inclusion is obtained. A hybrid inclusion interaction model has been used to compute the effective elastic properties of polyethylene. The model results are compared to experimental data to assess the capabilities of the two- or three-phase composite inclusion model.
\end{abstract}

\footnotetext{
${ }^{1}$ Reproduced from: A. Sedighiamiri, T.B. van Erp, G.W.M. Peters, L.E. Govaert, J.A.W. van Dommelen, Micromechanical Modeling of the Elastic Properties of Semicrystalline Polymers: a Three-Phase Approach, Journal of Polymer Science, Part B: Polymer Physics, 48: 2173-2184, 2010.
} 


\subsection{Introduction}

Semicrystalline polymeric materials are extensively used in structural, industrial and electronic applications. The mechanical properties of these materials strongly depend on their microstructure. Therefore, it is important to establish a relation between the microstructure of semicrystalline polymers and their effective mechanical properties. The elastic modulus of semicrystalline polymers is an important property and difficult to predict due to their dependence on many factors, such as: molecular weight, thermal history and crystallization conditions [1]. The reported elastic properties of a certain polymer show large scattering, because the samples used may differ from one another in terms of the percentage of crystallinity or the microstructural morphology. Such scattering can be seen in figure 2.1, which shows the Young's modulus of melt crystallized polyethylene versus the degree of crystallinity, reported by different sources.

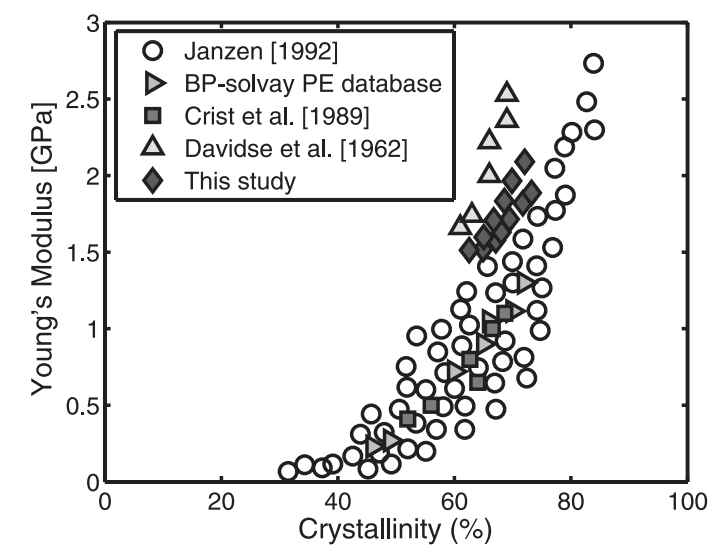

Figure 2.1 Measurements of Young's modulus as a function of crystallinity for polyethylene, reported by: Davidse et al. [2], Crist et al. [3], Janzen [4] and BP-Solvay polyethylene database [5].

Semicrystalline polymers can be considered as heterogeneous materials, and by using micromechanical models their overall mechanical properties can be estimated. In recent years, many studies have focused on the elastoplastic or elastoviscoplastic behavior of semicrystalline polymers. Lee et al. [6-8] developed a specific micromechanical model in which a rigid-viscoplastic composite inclusion model was used. Nikolov et al. [9, 10] used the same model for the small deformation behavior by assuming a viscoelastic behavior for the amorphous phase. They used a Sachs-inclusion model, which leads to a lower bound, underestimating the experimental moduli [11]. Van Dommelen et al. [12] have also used the Lee et al. approach for an elasto-viscoplastic model for large 
deformation of semicrystalline polymers. In this model, the behavior of the amorphous phase was assumed to be isotropic elastic with a rate-dependent plastic flow with strain hardening resulting from molecular orientation. The crystalline phase was modeled as anisotropic elastic with plastic flow occurring via crystallographic slip.

With most studies focusing on the (visco)plastic behavior of semicrystalline materials, only a few micromechanical modeling studies on the elastic behavior of these materials exist. Halpin and Kardos [13] proposed to use a HalpinTsai model in order to obtain the elastic moduli of semicrystalline polymers, thereby assuming that crystalline lamellae can be regarded as fibers. Phillips and Patel [14] applied this model to polyethylene. However, this model is generally used to calculate the moduli of short-fiber composites and is applicable for a low filler volume fraction, which is not the case for semi-crystalline materials, for which the crystallinity can often reach $60-70 \%$ [11].

Recently, Bedoui et al. [5, 11] proposed a differential scheme for the prediction of elastic moduli of semicrystalline polymers. In this model, the crystalline fraction is added step-by-step using a dilute scheme until the required volume fraction is reached. Pham and Tucker [15] used a three-phase model to predict the stiffness of polyethylene. The third phase, which is referred to as interphase or rigid-amorphous phase, is a region between the crystalline and amorphous phases, having a relatively ordered structure, constant thickness and due to the constraints from adjacent crystal lamellae [15], much higher stiffness than bulk amorphous phase. They compared their results with different experimental data and concluded that a three-phase model gives a better prediction compared to a two-phase model. In order to obtain the overall behavior, they used a Mori-Tanaka model [16]. This theory applies for volume fractions up to $30 \%$. Again, this is not the case for semicrystalline polymers, especially polyethylene with a crystallinity up to $70 \%$. Furthermore, they modeled the microstructure of the material as crystal lamellae randomly distributed in the amorphous matrix. However, in semicrystalline polymers crystalline lamellae are separated by amorphous layers [17]. Most recently, Gueguen et al. [18] also used a threephase model to estimate the effective elastic properties of PET (polyethylene terephthalate).

In this work, a two-phase and a three-phase layered composite inclusion model is developed in an elastic framework and compared in terms of predicting quantitatively the elastic properties of semicrystalline polymers, in particular HDPE. The incorporation of the interphase in the composite inclusion leads to a dependence of the overal properties on the lamellar thickness. A linear elastic composite inclusion model is used to obtain a closed form solution for the stiffness tensor of each inclusion. The semicrystalline polymer is considered as 
an aggregate of layered composite inclusions and a hybrid interaction scheme [6, 7. 12] is used. The predictions of the models are compared with experimental data to show the capability of the two and three-phase models. Semicrystalline polyethylene is considered as a case study. Data available in the literature is not very useful due to the lack of important data (crystallinity and lamellar thickness), the use of copolymers, etc. A well defined and well characterized material is required for a meaningful comparison between experiments and model results. Therefore, small and wide angle $\mathrm{X}$-ray scattering techniques are used to characterize the polyethylene samples in terms of both crystallinity and lamellar thickness. These are input for the composite inclusion model and make the comparison more accurate, whereas in other studies, the lamellar thickness was only chosen as a fitting parameter [15, 18]. It is also noteworthy that in this work it is attempted to establish a model which is based on a realistic morphological description of the semicrystalline polymers.

\subsection{Experimental}

The semicrystalline polymers used in this study are two different batches of HDPE of the same grade supplied by Sabic (Stamylan HD 9089S), with $T_{g} \ll$ $T_{\text {room }}, M_{W}=70,000[\mathrm{~g} / \mathrm{mol}]$ and $M_{n}=11,000[\mathrm{~g} / \mathrm{mol}]$.

\subsubsection{Sample preparation}

HDPE samples were compression molded between brass plates and aluminum foil at $200^{\circ} \mathrm{C}$. In order to obtain different levels of crystallinity and lamellar thickness, the samples were subjected to different crystallization procedures, namely quenching, for which molten samples were placed in a cold press, and isothermal crystallization (annealing), which was applied to quenched samples. For the isothermal crystallization procedure, HDPE samples were wrapped in aluminum foil and placed in an oven at different temperatures. The annealing of quenched samples results in an increase of the average long period $D(t)$, which is related to the distance between lamellae, according to [19]:

$$
D(t)=D_{0}+B(T) \ln \left[\frac{t}{t_{0}}\right],
$$

where $D_{0}$ is the initial long period of the unannealed sample at a corresponding positive but very small time $t_{0}$, and $B(T)$ is a constant which is a function of the annealing temperature $T$. 


\subsubsection{Crystallinity and lamellar thickness}

The degree of crystallinity and lamellar thickness of samples were measured by wide angle and small angle X-ray scattering experiments, respectively. The weight fraction of crystals of the semicrystalline samples is determined using:

$$
\mathcal{X}=\frac{C-A}{C}
$$

where $C$ is the area of the crystalline profile and $A$ is the area of the amorphous profile, which is impossible to measure separately due to the fast crystallization rate of PE. Therefore, an amorphous halo was estimated only [20].

The crystalline long period, $D$, was determined from the SAXS profile, which gives the intensity against the magnitude of the scattering vector $\vec{q}$, which is defined as [21]:

$$
q=\frac{2 \pi}{D}=\frac{4 \pi}{\lambda} \sin \theta
$$

where $D$ is the lamellar long period, $\lambda$ the $X$-ray wavelength and $\theta$ the scattering angle. The peak positions of the SAXS profiles are used to calculate the average lamellar thickness using:

$$
\delta^{c}=\mathcal{X}_{\mathrm{vol}} \cdot D,
$$

where $\mathcal{X}_{\mathrm{vol}}$ is the volumetric degree of crystallinity obtained from the weight fraction of crystals $\mathcal{X}$ :

$$
\mathcal{X}_{\mathrm{vol}}=\frac{\frac{\mathcal{X}}{\rho_{c}}}{\frac{\mathcal{X}}{\rho_{c}}+\frac{100-\mathcal{X}}{\rho_{a}}} \times 100 \%,
$$

with $\rho_{c}=1000 \mathrm{~kg} / \mathrm{m}^{3}$, the crystal density and $\rho_{a}=855 \mathrm{~kg} / \mathrm{m}^{3}$, the amorphous density [22]. Figure 2.2 shows the lamellar thickness and the interlamellar thickness of the samples versus degree of crystallinity. It can be seen that, in this range of crystallinity, lamellar thickness varies considerably with the degree of crystallinity, whereas interlamellar thickness remains almost constant.

\subsubsection{Mechanical testing}

Tensile tests on the dumbbell-shaped tensile specimens were performed at room temperature using a Zwick/Roell Z010 universal tensile tester, equipped with an 


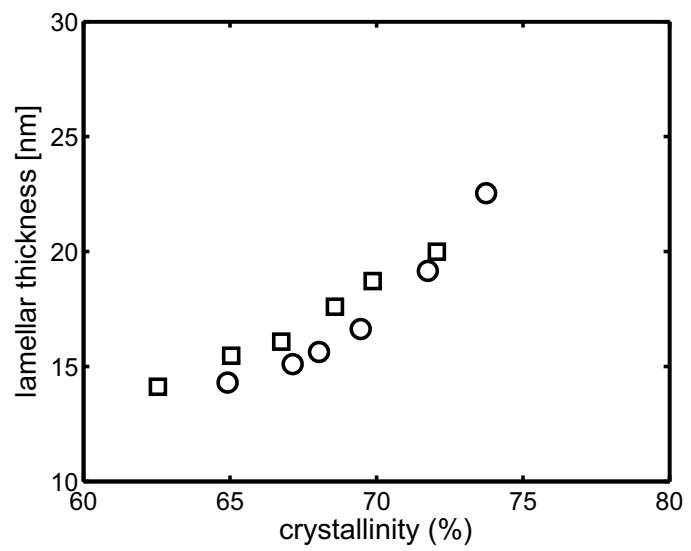

a

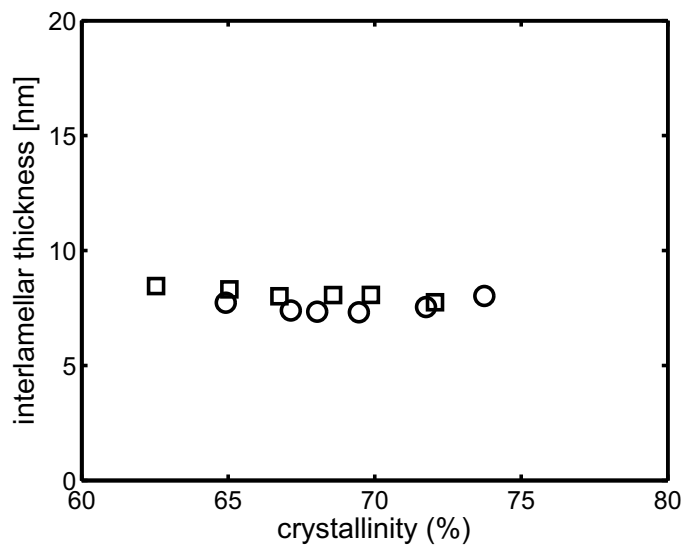

b

Figure 2.2 Variation of (a): lamellar thickness and (b) interlamellar thickness versus crystallinity of PE samples. Symbols show two different batches of the same grade.

uniaxial extensometer.

\subsection{Micromechanical modeling}

When cooled from the melt, many polymers, such as polyethylene, develop a semicrystalline structure. These materials often show a spherulitic morphology [23, 24]. Each spherulite consists of a radial assembly of twisted thin crystalline lamellae which are separated by amorphous layers [17], as shown in figure 2.3. In this section, a micromechanical model based on a layered structure is formulated in a linear elastic framework.

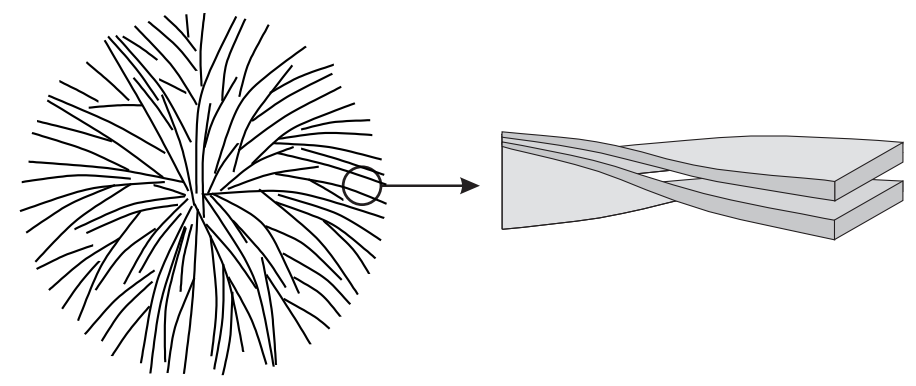

Figure 2.3 Morphology of a spherulitic semicrystalline polymer. 


\subsubsection{Two-phase composite inclusion}

The elastic behavior of microscopically heterogeneous semicrystalline material can be modeled by an aggregate of layered two-phase composite inclusions, as proposed by Lee et al. [6-8] for rigid-viscoplastic material behavior. Each composite inclusion is represented by a crystalline lamella which is mechanically coupled to its adjacent amorphous layer, as shown in figure 2.4. The stress and

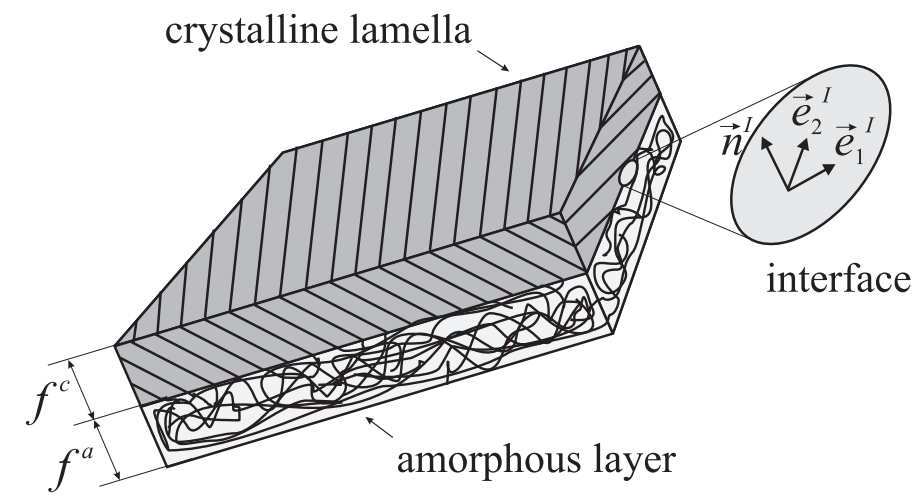

Figure 2.4 Schematic representation of a two-phase composite inclusion.

strain fields in each separate phase of the composite inclusion are assumed to be piecewise homogeneous; but, they can be different between the two coupled phases [12]. The volume fractions of the crystalline and amorphous phases are given by $f^{c}$ and $f^{a}=1-f^{c}$, respectively. Otherwise, the shapes and dimensions of these layers are not specified. The inclusion-averaged stress tensor $\sigma$ and linear strain tensor $\epsilon$ are given by the volume average over the two phases:

$$
\begin{aligned}
& \sigma^{I}=f^{c} \sigma^{c}+\left(1-f^{c}\right) \sigma^{a}, \\
& \boldsymbol{\epsilon}^{I}=f^{c} \boldsymbol{\epsilon}^{c}+\left(1-f^{c}\right) \boldsymbol{\epsilon}^{a},
\end{aligned}
$$

where the superscript " $I$ " denotes the composite inclusion. The two phases are assumed to be kinematically compatible and in equilibrium across the interface. The traction continuity and compatibility condition on the interface can be written as:

$$
\begin{aligned}
& \sigma^{a} \cdot \vec{n}^{I}=\sigma^{c} \cdot \vec{n}^{I}=\sigma^{I} \cdot \vec{n}^{I}, \\
& \vec{e}_{1}^{I} \cdot \epsilon^{a} \cdot \vec{e}_{2}^{I}=\vec{e}_{1}^{I} \cdot \epsilon^{c} \cdot \vec{e}_{2}^{I}=\vec{e}_{1}^{I} \cdot \epsilon^{I} \cdot \vec{e}_{2}^{I},
\end{aligned}
$$


where $\vec{n}^{I}=\vec{e}_{3}^{I}$ denotes the unit normal vector of the interface, and $\vec{e}_{1}^{I}$ and $\vec{e}_{2}^{I}$ are two unit vectors in the interface such that $\vec{e}_{1}^{I} \times \vec{e}_{2}^{I}=\vec{e}_{3}^{I}$. For convenience, let the following fourth-order subspace projection tensors, based on the orientation of the amorphous/crystalline interface plane, be defined as:

$$
\begin{aligned}
& { }^{4} \mathcal{P}_{x}^{I}=\sum_{i=1}^{2} \sum_{j=1}^{2} \vec{e}_{i}^{I} \otimes \vec{e}_{j}^{I} \otimes \vec{e}_{j}^{I} \otimes \vec{e}_{i}^{I}, \\
& { }^{4} \mathcal{P}_{n}^{I}={ }^{4} \mathcal{I}-{ }^{4} \mathcal{P}_{x}^{I},
\end{aligned}
$$

where ${ }^{4} \mathcal{I}$ is the fourth-order identity tensor. Then, the interface conditions can be rewritten as:

$$
\begin{aligned}
& { }^{4} \mathcal{P}_{n}^{I}: \sigma^{a}={ }^{4} \mathcal{P}_{n}^{I}: \sigma^{c}={ }^{4} \mathcal{P}_{n}^{I}: \sigma^{I}, \\
& { }^{4} \mathcal{P}_{x}^{I}: \boldsymbol{\epsilon}^{a}={ }^{4} \mathcal{P}_{x}^{I}: \epsilon^{\mathcal{c}}={ }^{4} \mathcal{P}_{x}^{I}: \boldsymbol{\epsilon}^{I} .
\end{aligned}
$$

By using consistency equations (2.6) and (2.7), together with interface conditions (2.12) and (2.13), the volume-averaged fourth-order stiffness tensor of an inclusion can be obtained (for more details, see appendix 2.A).

\subsubsection{Towards a three-phase model}

Semicrystalline polymers have been modeled as two-phase composites [9-12], consisting of crystalline lamellae and an interlamellar matrix. In these models, the properties of the interlamellar phase are assumed to be equal to those of the bulk amorphous phase. The interlamellar stiffness, therefore, is taken to be constant for all values of crystallinity. However, some literature suggest that the interlamellar properties vary with the degree of crystallinity [3, 15, 25]. Strobl and Hagedorn [26] first used Raman spectroscopy to characterize the three-phase morphological structure of semicrystalline polyethylene. They described semicrystalline polyethylene as a superposition of three components: an orthorhombic crystalline phase, an isotropic amorphous phase and a third phase consisting of anisotropic ordered chain segments, which are oriented but not in orthorhombic packing. The crystalline fraction obtained by using the three-phase analysis was smaller than that derived from density. Lin et al. [27] showed that the crystallinities measured by DSC and density, reflect the summation of orthorhombic crystalline fraction and some non-orthorhombic interphase fraction.

Lamellar thickness varies with crystallinity [20, 28, 29], see also the experimental 
results in figure 2.2 a. In order to take into account the variation of lamellar thickness with the degree of crystallinity, a three-phase model is proposed. A composite inclusion is assumed to consist of a layered arrangement of a crystalline lamella, a so-called rigid-amorphous phase and a bulk amorphous phase, see figure 2.5. Each crystalline lamella is surrounded by two rigidamorphous layers, each of which has a thickness of $\delta^{r}$. Consequently, four layers are present in the schematic representation of this three-phase model.

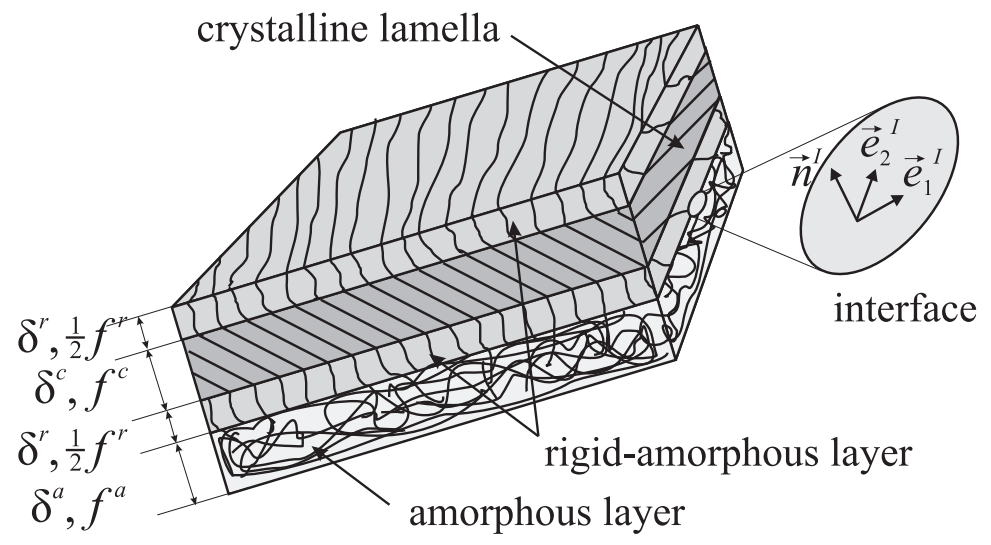

Figure 2.5 Schematic representation of a three-phase composite inclusion.

A relationship between the crystallinity and the volume fractions of the other two phases can be obtained:

$$
\begin{aligned}
& f^{r}=2 \frac{\delta^{r}}{\delta^{a}} f^{a}, \\
& f^{a}=\frac{1-f^{c}}{1+2 \frac{\delta^{r}}{\delta^{a}}} .
\end{aligned}
$$

A constant rigid-amorphous thickness is assumed and this thickness is obtained from experiments. The geometrical configuration is then determined by the ratio of rigid-amorphous layer thickness and the amorphous layer thickness. The lamellar thickness varies with the crystallinity as:

$$
\delta^{c}=\delta^{i l} \frac{f^{c}}{1-f^{c}}
$$

with $\delta^{i l}=\delta^{a}+2 \delta^{r}$, the interlamellar thickness. In figure 2.6, situations with different ratios of rigid-amorphous layer thickness and amorphous layer thickness are shown. 


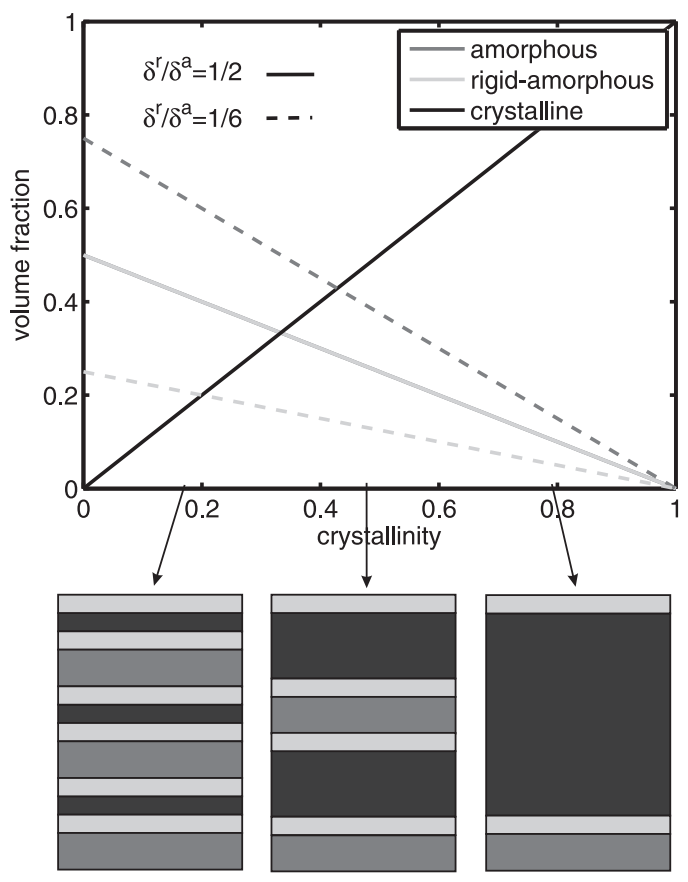

a

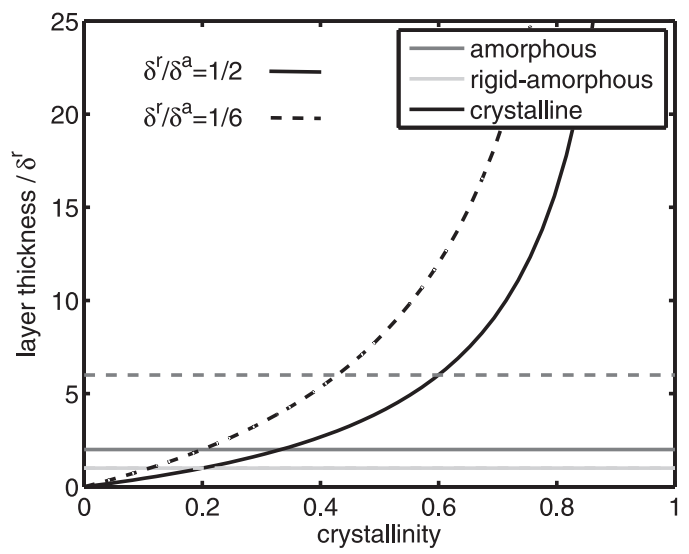

b

Figure 2.6 (a): Relationship between the crystallinity and the volume fractions of the two other phases. (b): variation of phases thicknesses with crystallinity.

Consistency conditions, for the case of a three-phase composite inclusion, can be written as:

$$
\begin{aligned}
& \sigma^{I}=f^{c} \sigma^{c}+f^{r} \sigma^{r}+f^{a} \sigma^{a}, \\
& \boldsymbol{\epsilon}^{I}=f^{c} \boldsymbol{\epsilon}^{c}+f^{r} \boldsymbol{\epsilon}^{r}+f^{a} \boldsymbol{\epsilon}^{a},
\end{aligned}
$$

and interface conditions are governed by:

$$
\begin{gathered}
{ }^{4} \mathcal{P}_{n}^{I}: \sigma^{a}={ }^{4} \mathcal{P}_{n}^{I}: \sigma^{r}={ }^{4} \mathcal{P}_{n}^{I}: \sigma^{\mathcal{C}}={ }^{4} \mathcal{P}_{n}^{I}: \sigma^{I}, \\
{ }^{4} \mathcal{P}_{x}^{I}: \boldsymbol{\epsilon}^{a}={ }^{4} \mathcal{P}_{x}^{I}: \boldsymbol{\epsilon}^{r}={ }^{4} \mathcal{P}_{x}^{I}: \boldsymbol{\epsilon}^{\mathcal{c}}={ }^{4} \mathcal{P}_{x}^{I}: \boldsymbol{\epsilon}^{I},
\end{gathered}
$$

where the fourth order projection tensors, ${ }^{4} \mathcal{P}_{n}^{I}$ and ${ }^{4} \mathcal{P}_{x}^{I}$, are defined as in equations (2.10) and (2.11). The volume-averaged fourth-order stiffness tensor of a three-phase composite inclusion can be obtained in the same way as for the two-phase composite inclusion and is a function of the elastic moduli of the three phases as well as their volume fractions (see appendix 2.B). Then, 
the semicrystalline polymer is modeled as an aggregate of these three-phase layered composite inclusions. The three-phase model incorporates the ratio of the rigid-amorphous and amorphous layer thicknesses, whereas no length scale is included in the two-phase model. The average stiffness of the interlamellar material is, therefore, no longer constant.

\subsubsection{Inclusion interaction law}

To relate the volume-averaged mechanical behavior of each layered composite inclusion to the aggregate of composite inclusions, a local-global interaction law must be formulated. Consider an aggregate of $N$ composite inclusions. The equivalent homogeneous elastic behavior of the aggregate is defined by:

$$
\bar{\sigma}={ }^{4} \overline{\mathcal{C}}: \overline{\boldsymbol{\epsilon}}
$$

where $\bar{\sigma}$ and $\overline{\boldsymbol{\epsilon}}$ denote the macroscopic stress and strain tensors. The consistency conditions for the aggregate are written as:

$$
\begin{aligned}
& \bar{\sigma}=\left\langle\sigma^{I^{i}}\right\rangle, \\
& \overline{\boldsymbol{\epsilon}}=\left\langle\epsilon^{I^{i}}\right\rangle,
\end{aligned}
$$

with the notation $\langle\cdot\rangle=\sum_{i=1}^{N} f^{I^{i}}(\cdot)^{i}$ denoting the volume average of all inclusions and $f^{I^{i}}$ the volume fraction of composite inclusion $i$. In case the size of all composite inclusions is the same, $f^{I^{i}}$ equals $\frac{1}{N}$. Based on a specific assumption for the interactions between the inclusions, several interaction laws can be used to estimate the properties of an aggregate of inclusions.

The hybrid-interaction model for lamellar composites, which was introduced by Lee et al. [6-8] constitutes an intermediate approach between the upper bound Voigt- and the lower bound Reuss-inclusion models.

Since the interface condition (2.19) acts upon the ${ }^{4} \mathcal{P}_{n}^{I^{i}}$ subspace projection of $\sigma^{I^{i}}$, and provides certain constraints on these components of the inclusionaveraged stress, a Voigt-like interaction law is assumed for the ${ }^{4} \mathcal{P}_{n}^{I^{i}}$ subspace projections of the inclusion-averaged strain. Furthermore, since a certain measure of compatibility is provided for the inclusion-averaged strain by the interface condition (2.20), acting on the ${ }^{4} \mathcal{P}_{x}^{I^{i}}$ subspace projections of $\boldsymbol{\epsilon}^{I^{i}}$, a Reuss-like interaction law is assumed for the corresponding ${ }^{4} \mathcal{P}_{x}^{I^{i}}$ subspace projection of the inclusion-averaged stress [12]. 
For the hybrid interaction model, in contrast to Voigt- and Reuss-inclusion models, neither conditions (2.22) or (2.23) are satisfied trivially. In order to satisfy the 12 conditions of consistency, six more auxiliary unknowns are introduced. Different choices for these auxiliary unknowns can be made, leading to different versions of the hybrid interaction model. A deformation-like auxiliary unknown was found to be the most suitable and the resulting hybrid interaction model was showed to provide the most realistic predictions [6-8, 12]. In the $\hat{\epsilon}$-inclusion model, an unknown auxiliary strain field $\hat{\boldsymbol{\epsilon}}$ is introduced. Then, a Reusslike interaction law is applied to the ${ }^{4} \mathcal{P}_{x}^{I^{i}}$ subspace projection of $\sigma^{I^{i}}$. The subspace projection ${ }^{4} \mathcal{P}_{n}^{I^{i}}$ of the inclusion-averaged strain is assumed to equal the corresponding subspace projection of $\hat{\boldsymbol{\epsilon}}$ :

$$
\begin{array}{ll}
{ }^{4} \mathcal{P}_{x}^{I^{i}}: \sigma^{I^{i}}={ }^{4} \mathcal{P}_{x}^{I^{i}}: \bar{\sigma} \quad ; & i=1, \ldots, N, \\
{ }^{4} \mathcal{P}_{n}^{I^{i}}: \boldsymbol{\epsilon}^{I^{i}}={ }^{4} \mathcal{P}_{n}^{I^{i}}: \hat{\boldsymbol{\epsilon}} & ;
\end{array}
$$

Considering a system of $N$ inclusions, subjected to a macroscopic stress field, $3 N$ local unknowns $\left({ }^{4} \mathcal{P}_{n}^{I^{i}}: \sigma^{I^{i}}\right)$ and 6 global unknowns $\hat{\boldsymbol{\epsilon}}$, must be obtained to determine the state of each composite inclusion. Equation (2.25) provides $3 N$ equations. Furthermore, the macroscopic equilibrium condition $\bar{\sigma}=\left\langle\sigma^{I^{i}}\right\rangle$, provides six more equations, leading to a set of a $(3 N+6)$ linear equations to be solved. Elimination of these unknowns leads to a direct formulation for the fourth order elasticity tensor of the aggregate, see Appendix 2.C.

\subsection{Characterization of constituent phases}

In this work, isotropic polyethylene (PE) is studied as an important example of semicrystalline polymers. Experimental studies of melt-crystalized PE and molecular models show that lamellar surfaces are of the $\{h 0 l\}$ type, where the angle between the chain direction $\vec{c}$ and the lamellar normal direction $\vec{n}$ varies between $20^{\circ}$ and $40^{\circ}$ [23, 30]. Here, the lamellar surface is set at $\{201\}$, which corresponds with an angle of $35^{\circ}$. The spherulitic structure of melt-crystalized PE is represented by an aggregate of randomly oriented inclusions. In the following the characteristics of the constituent phases, namely the crystalline phase, the amorphous region and the rigid-amorphous phase are described. 


\subsubsection{Crystalline phase}

The crystalline phase possesses highly anisotropic properties with a high elastic modulus in the chain direction. PE crystals have an orthorhombic structure, with lattice parameters $a=7.39 \AA, b=4.95 \AA$ and $c=2.54 \AA$ [31, 32].

For orthorhombic PE crystals, the elasticity matrix $\underline{\mathcal{C}}$ involves nine independent elastic constants:

$$
\underline{\mathcal{C}}=\left[\begin{array}{cccccc}
c_{11} & c_{12} & c_{13} & 0 & 0 & 0 \\
c_{12} & c_{22} & c_{23} & 0 & 0 & 0 \\
c_{13} & c_{23} & c_{33} & 0 & 0 & 0 \\
0 & 0 & 0 & c_{44} & 0 & 0 \\
0 & 0 & 0 & 0 & c_{55} & 0 \\
0 & 0 & 0 & 0 & 0 & c_{66}
\end{array}\right]
$$

and relates stress and strain, which are here stored in the following order:

$$
\begin{aligned}
& \underset{\sim}{\sigma}=\left[\begin{array}{llllll}
\sigma_{11} & \sigma_{22} & \sigma_{33} & \sigma_{12} & \sigma_{13} & \sigma_{23}
\end{array}\right]^{T}, \\
& \underset{\sim}{\epsilon}=\left[\begin{array}{llllll}
\epsilon_{11} & \epsilon_{22} & \epsilon_{33} & 2 \epsilon_{12} & 2 \epsilon_{13} & 2 \epsilon_{23}
\end{array}\right]^{T} .
\end{aligned}
$$

The elastic constants of the PE unit cell have theoretically been studied by several authors [33-36]. In addition, experimental estimates for these parameters have been obtained based on drawn material [37]. Table 2.1 shows the results of the theoretically calculated elastic constants, obtained by different authors and the only available experimentally measured values to our knowledge. In addition to the choice of intermolecular force field parameters, theoretical results are also sensitive to the setting angle, which is the angle between the planar zigzag polyethylene chain with the $b$-axis of the orthorhombic unit cell. Another important factor is the intermolecular interaction, which is very sensitive to intermolecular distance. Thus, the elastic constants can be significantly affected by temperature [38]. According to table [2.1, except for the differences due to the mentioned factors, the principal features of the anisotropy can be observed in all theoretical calculations, with a high value for $c_{33}$, which is the elastic constant along the chain direction [39]. Choy and Leung [37] used an ultrasonic method for measurement of the elastic constants of ultradrawn high-density PE with a draw ratio up to $\lambda=27$. This draw ratio orients the chains toward the drawing axis, but does not lead to fully drawn samples. The result is a value of $c_{33}=81 \mathrm{GPa}$, which is less than the theoretical results. However, Tashiro et al. [40] showed that in polymers with a planar zigzag conformation, the chain experiences a thermal motion at room temperature and contracts to some extent 
Table 2.1 Elastic constants for polyethylene obtained in different studies.

\begin{tabular}{|c|ccc|c|}
\hline \multirow{3}{*}{$c_{i j}$ (GPa) } & \multicolumn{3}{|c|}{ Theoretical } & Experimental \\
\cline { 2 - 5 } & $\begin{array}{c}\text { Odajima } \\
\text { and Maeda [33] }\end{array}$ & $\begin{array}{c}\text { Karasawa } \\
\text { et al. [34] }\end{array}$ & $\begin{array}{c}\text { Tashiro } \\
\text { et al. [36] }\end{array}$ & $\begin{array}{c}\text { Choy } \\
\text { and Leung [37] }\end{array}$ \\
\hline$c_{11}$ & 4.83 & 8.3 & 7.99 & 7.0 \\
$c_{22}$ & 8.71 & 8.2 & 9.92 & 7.0 \\
$c_{33}$ & 257.1 & 318.4 & 315.92 & 81 \\
$c_{12}$ & 1.16 & 4.3 & 3.28 & 3.8 \\
$c_{13}$ & 2.55 & 0.7 & 1.13 & 4.7 \\
$c_{23}$ & 5.84 & 2.5 & 2.14 & 3.8 \\
$c_{44}$ & 2.06 & 3.6 & 3.62 & 1.6 \\
$c_{55}$ & 0.78 & 1.7 & 1.62 & 1.6 \\
$c_{66}$ & 2.83 & 3.0 & 3.19 & 1.6 \\
\hline
\end{tabular}

from the planar-zigzag conformation, which induce a drop in the modulus along the chain direction. Furthermore, the values of theoretical calculations, especially for the elastic constant along the chain direction, cannot be reached in practice, since semicrystalline polymers are by no means perfect from a structural point of view. The crystals in semicrystalline polymers contain imperfections such as dislocations or point defects, leading to some degree of disorder in the polymer crystals and distortion of the crystal lattice [39].

Considering the lack of sufficient experimental measurements in the literature to fully determine nine elastic constants of a PE unit cell and the drawbacks of theoretical calculations, results with a theoretically calculated and experimentally obtained stiffness are presented. Here, the experimental constants obtained by Choy and Leung [37] as well as the theoretical stiffness constants calculated by Tashiro et al. [36] are used.

\subsubsection{Amorphous phase}

For PE, the glass transition temperature of the amorphous phase is below the room temperature. The amorphous phase, therefore, is in the rubbery state at room temperature. The following equation relates the shear modulus of the rubber plateau $G_{N}^{0}$ to the molecular mass between entanglements and can be applied to the amorphous phase of thermoplastic polymers above the glass 
transition temperature [41]:

$$
G_{N}^{0}=\frac{\rho R T}{M_{e}}
$$

with $\rho$ the amorphous phase density, $R$ the ideal gas constant, $T$ the temperature and $M_{e}$ the molecular mass between entanglements. The shear modulus of the rubber plateau can be obtained by rheological measurements at an elevated temperature and is reported to be almost $2 \mathrm{MPa}$ for $\mathrm{PE}$ at approximately $463 \mathrm{~K}$ [41-43]. Since PE cannot be obtained in a fully amorphous state at room temperature, $G_{N}^{0}$ values are scaled to the ambient temperature through [44]:

$$
G_{N}^{0}(T)=G_{N}^{0}\left(T_{0}\right) \frac{\rho T}{\rho_{0} T_{0}}
$$

with $T_{0}$ the reference temperature and $T$ the ambient temperature, which is set to be $298 \mathrm{~K}$. The amorphous phase density at $463 \mathrm{~K}$ and ambient temperature for PE are $760 \mathrm{~kg} / \mathrm{m}^{3}$ and $855 \mathrm{~kg} / \mathrm{m}^{3}$, respectively [11, 22]. Then, the Young's modulus $E$ and the Poisson's ratio $v$ of the amorphous phase are obtained, assuming a bulk modulus $K$ of the amorphous phase equal to $3000 \mathrm{MPa}$ [5]. The mechanical properties used for the amorphous phase are listed in Table 2.2.

Table 2.2 Mechanical properties of the amorphous phase of PE.

\begin{tabular}{cc}
\hline$E(\mathrm{MPa})$ & $v(-)$ \\
\hline 4.5 & 0.49975 \\
\hline
\end{tabular}

\subsubsection{Rigid-amorphous phase}

The mechanical properties of the interlamellar phase (amorphous phase + rigidamorphous phase) play a significant role for the macroscopic properties of semicrystalline polymers. Unfortunately, no experimental measurements of the rigid-amorphous properties, such as thickness and stiffness, exist because of its small dimension. Furthermore, the stiffness may vary throughout the thickness of the layer. Recently, Veld et al. [25] estimated the thermoelastic properties of the noncrystalline domain (interlamellar phase) of semicrystalline PE by using molecular simulations. The results show that the noncrystalline interlamellar phase is anisotropic and exhibits properties intermediate between that of the semicrystalline solid and of the amorphous melt. They also determined the 
interface thickness $\delta^{r}$ as a function of temperature, ranging from $350 \mathrm{~K}$ to 450 $\mathrm{K}$, to be $1 \mathrm{~nm}$ to $2 \mathrm{~nm}$.

Here, the interlamellar phase is assumed to be isotropic. The rigid-amorphous phase is, therefore, considered to be isotropic as well so that it can be described with only two elastic parameters. The bulk modulus $K^{r}$ is set equal to 5000 $\mathrm{MPa}$, which is between the bulk modulus of the crystalline lamellae and that of the amorphous phase. In this work, the shear modulus of the rigid-amorphous phase, $G^{r}$, is considered as a fitting parameter. The influence of the ratio $\frac{\delta^{r}}{\delta^{a}}$ and $G^{r}$ on the macroscopic results will be investigated.

\subsection{Results and discussion}

The sensitivity of the macroscopic elastic modulus of PE, calculated by using twophase and three-phase micromechanical models to various physical modeling parameters is investigated and results are compared to experimental data. For micromechanical modeling of the spherulitic semicrystalline polymers, the number of randomly oriented inclusions within the aggregate, $N$, should be sufficiently high in order to ensure isotropy. In order to investigate the influence of the aggregate size on the $\hat{\boldsymbol{\epsilon}}$-inclusion model, aggregates of different sizes $N$, have been randomly generated. For each aggregate size $N, 20$ different configurations have been used to calculate the effective elastic properties at $70 \%$ crystallinity from:

$$
\bar{E}=\frac{1}{3}\left(\frac{1}{\bar{s}_{11}}+\frac{1}{\bar{s}_{22}}+\frac{1}{\bar{s}_{33}}\right),
$$

with $\bar{s}_{i i}$ the components of the macroscopic compliance matrix. Figure 2.7 shows the average of the 20 homogenizations and the corresponding standard deviation bandwidth. It can be observed that for large aggregate sizes, the average stiffness converges and the corresponding standard deviation approaches zero. In the following, 2000 randomly generated inclusions are used to achieve an isotropic result and to minimize statistical variations.

First, in order to quantitatively assess the capabilities of the two-phase composite inclusion model, the results are compared to the experimental data. Although the elastic moduli measured for the PE system used in this study may appear high, they are in the range of experimental data in literature (see figure 1). Figure 2.8 shows the predicted Young's modulus of PE samples by using the two-phase $\hat{\boldsymbol{\epsilon}}$ inclusion model with different PE unit cell stiffnesses. As already mentioned, it is difficult, if not impossible, to accurately measure 9 elastic constants of the PE 


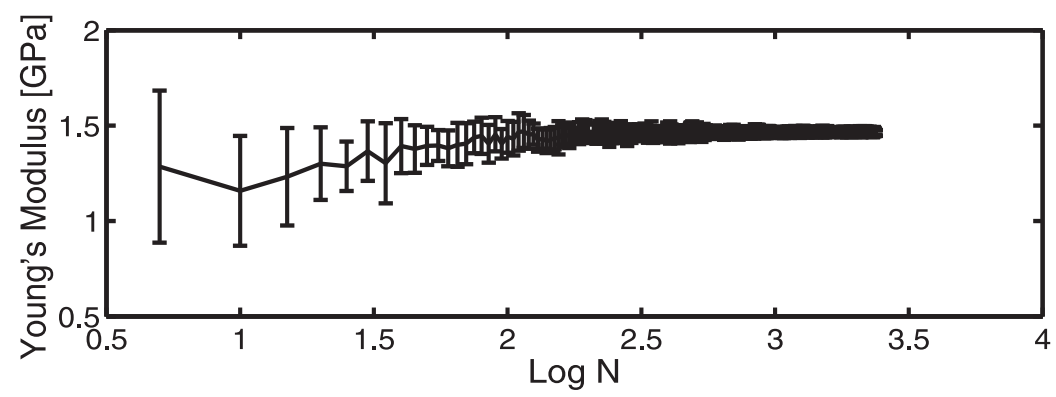

Figure 2.7 Effective elastic properties of spherulitic PE at 70\% of crystallinity using the two-phase model, with different aggregate sizes.

unit cell. On the other hand, theoretical calculations are based on the assumption of a perfect crystalline structure, which may not be correct. In order to reveal also the effect of specific interactions among inclusions on the macroscopic results, the two extreme inclusion interaction models, namely Voigt and Reuss models, are also shown. Although the theoretical stiffness in the chain direction is almost four times bigger than the experimental stiffness in the chain direction, it is observed that the interaction between inclusions plays a far more important role in the predictions. The two-phase $\hat{\boldsymbol{\epsilon}}$-inclusion model predicts the Young's modulus of experimental data rather well in contrast to the classical interaction models, especially when using the theoretically obtained $E^{c}$. However, there are some limitations to the two-phase model. It was mentioned before that a two-phase

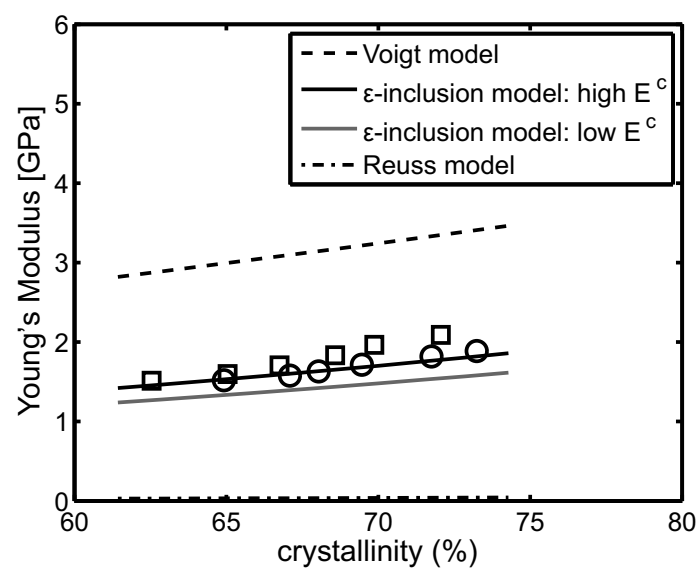

Figure 2.8 Predicted Young's modulus of PE samples using the two-phase model with different interaction laws. Symbols give the experimental results for the two batches.

composite inclusion model does not incorporate any length scale and, therefore, the predicted stiffness is independent of lamellar or interlamellar thickness. 
The three-phase model incorporates an internal length scale through amorphous layer and interphase thicknesses. Now, the influence of various physical parameters of the rigid amorphous phase in the three-phase model is systematically investigated using the theoretical stiffness for the crystalline phase. For this purpose, it is assumed that interlamellar thickness $\delta^{i l}$ remains constant with the degree of crystallinity in the range of $60 \%-80 \%$. The value for interlamellar thickness is taken from the experimentally measured interlamellar thickness vs. crystallinity in figure 2.2, i.e. $\delta^{i l}=8 \mathrm{~nm}$.

In this work the elastic modulus of the rigid-amorphous phase is considered as a fiting parameter. The influence of varying this parameter from that of fully amorphous to that of fully crystalline material on the macroscopic results is illustrated in figure 2.9. The prediction of the three-phase model becomes equal to that of the two-phase model in case the properties of the rigid-amorphous phase are considered the same as the bulk amorphous phase. Considering the stiffness of the rigid-amorphous phase as high as that of the crystalline phase, also leads to a high value for the macroscopic Young's modulus, corresponding to a higher effective crystallinity. Therefore it can be seen that the mechanical properties of the rigid-amorphous phase play an important role in the macroscopic properties. The macroscopic Young's modulus is also effected by the rigid-amorphous layer thickness, as the ratio $\delta^{r} / \delta^{a}$ influences the volume fraction of the rigidamorphous phase, see figure 2.10. The thicker the rigid-amorphous layer, the stiffer the interlamellar phase and, in consequence, the material.

It is observed that the three-phase model enables having different configurations with the same degree of crystallinity, through different rigid-amorphous layer thicknesses and stiffness, while this effect is not captured with the two-phase model. However, for HDPE it is clear that for isotropic material a two-phase model is sufficient, i.e. although a rigid-amorphous phase may be present, its relative influence is minimal for constant interlamellar thickness. Therefore, by using a two-phase model and changing the properties of amorphous phase to the combined properties of the rigid-amorphous region and the amorphous region, one can almost obtain the results of the three-phase model with a given rigid-amorphous layer thickness and stiffness. However, although the prediction of the macroscopic properties would be the same for the isotropic case, for oriented systems, the results can be considerably different. Figure 2.11 shows equal area projection pole figures of an oriented system with a row structure and uniaxial orientation of surface normals. Here, the lamellar surface is set at $\{102\}$, which corresponds with an angle of $10^{\circ}$ between the chain direction $\vec{c}$ and the lamellar normal direction $\vec{n}$. In such a system, the Young's modulus in the orientation direction will be dominated by the properties of the amorphous phase. Figure 2.12 shows the prediction of two-phase and three-phase models 


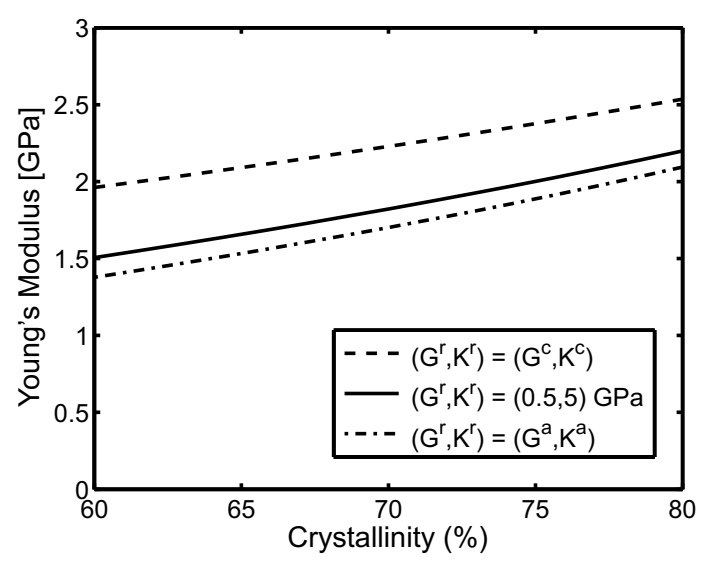

Figure 2.9 Effect of different shear and bulk moduli of the rigid-amorphous layer $G^{r}$ and $K^{r}$, on the prediction of the three-phase composite inclusion model with $\delta^{r}=1 \mathrm{~nm}$.

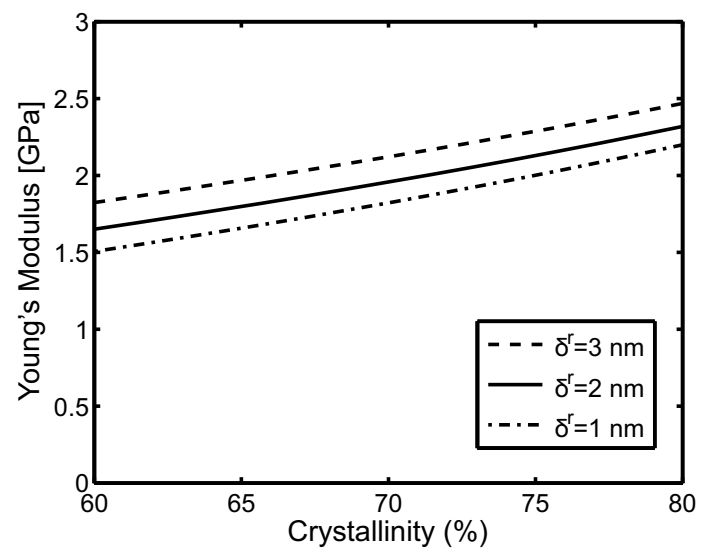

Figure 2.10 Effect of different rigid-amorphous layer thickness on the prediction of the three-phase composite inclusion model with $G^{r}=0.5 \mathrm{GPa}$.

for isotropic and oriented systems. As can be seen, it is possible to increase the stiffness of the amorphous phase in the two-phase model $\left(E^{a}=10 \mathrm{MPa}\right)$, so that it can reproduce the results of the three-phase model with $\delta^{r}=2 \mathrm{~nm}$, in isotropic systems. However, for oriented systems, in which the amorphous phase is dominant in the out of plane modulus $\bar{E}_{33}$, higher stiffness of the amorphous phase influences the macroscopic results considerably, making it impossible to reproduce the results of the three-phase model.

These results show that in order to investigate the mechanical properties of a rigid-amorphous phase and its influence on the mechanical properties of a semicrystalline polymer, experimental results of the same system with different 


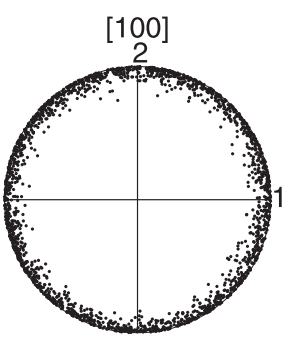

a

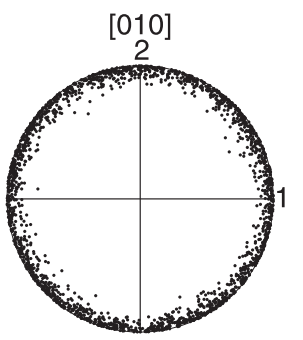

$\mathrm{b}$

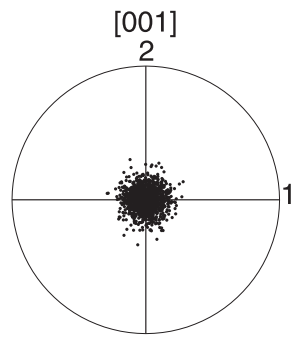

C

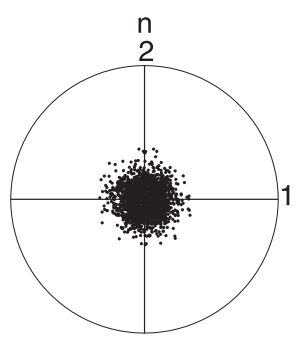

d

Figure 2.11 Equal area projection pole figures of uniaxially oriented (a)-(c) crystallographic lattice directions and $(\mathrm{d})$ interface normals

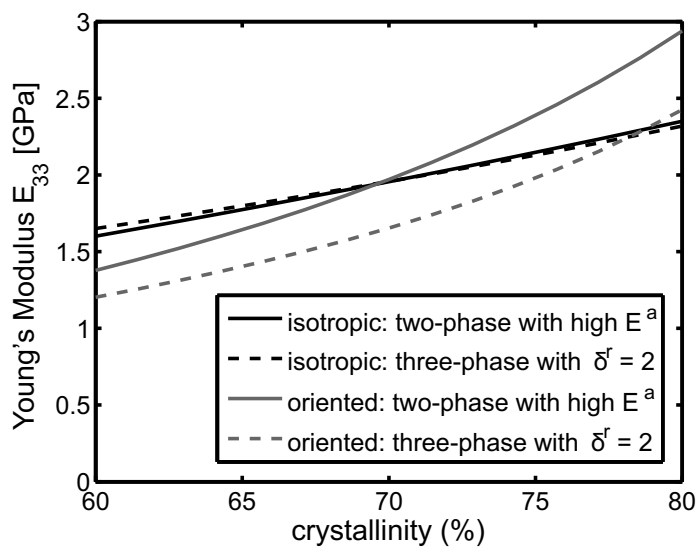

Figure 2.12 Effect of changing the properties of amorphous phase to the combined properties of the rigid-amorphous region and the amorphous region in isotropic and oriented systems.

morphological configurations are needed. For isotropic systems, a varying interlamellar spacing with constant crystallinity will show the influence of the rigid-amorphous phase. Furthermore, a comparison of the stiffness of both isotropic and oriented systems elucidate the influence of incorporation of the rigid-amorphous phase as a separate constituent of the semicrystalline microstructure. In addition, the effects of the rigid-amorphous phase may be more pronounced in other systems such as LDPE.

\subsection{Conclusion}

A micromechanical model has been developed for the prediction of the effective mechanical properties of semicrystalline polymers. Both two-phase and three- 
phase layered composite inclusions have been used as representative microstructural elements, based on the lamellar structure that is commonly observed in semi-crystalline polymers. All constitutive phases are mechanically coupled by enforcing compatibility and equilibrium conditions on the interface between the crystalline lamella, the interphase and the amorphous layer. A hybrid interaction model also has been used to obtain the effective elastic properties, namely the elastic $\hat{\boldsymbol{\epsilon}}$-inclusion model, which provides an intermediate approach. Comparing the predicted Young's modulus to experimental data, shows that the two-phase layered composites model together with the elastic $\hat{\boldsymbol{\epsilon}}$-inclusion model provides a good prediction. However, there are some limitations to the two-phase model.

It has been revealed that a three-phase composite inclusion model enables to incorporate an internal length scale, leading to have different configurations with the same degree of crystallinity, through different rigid-amorphous layer thicknesses and stiffness, while this effect was not captured with the two-phase model. It is noteworthy that more experimental data of a system with different morphological configurations are needed to correctly verify the influence of the mechanical properties of the rigid-amorphous phase on the macroscopic properties. 


\section{A Appendix: Stiffness tensor of an elastic two-phase composite inclusion}

In this appendix, a closed form solution for the average elastic properties of a two-phase composite inclusion with orientation $\vec{n}^{I}$ is presented. The constitutive behavior of each phase is given by:

$$
\sigma^{\pi}={ }^{4} \mathcal{C}^{\pi}: \epsilon^{\pi}, \quad \text { with } \quad \pi=a, c,
$$

with ${ }^{4} \mathcal{C}^{\pi}$ the fourth order elasticity tensor of phase $\pi$. The consistency conditions are written as:

$$
\begin{aligned}
& \sigma^{I}=f^{c} \sigma^{c}+\left(1-f^{c}\right) \sigma^{a}, \\
& \epsilon^{I}=f^{c} \epsilon^{c}+\left(1-f^{c}\right) \epsilon^{a},
\end{aligned}
$$

and the interface conditions are given by:

$$
\begin{gathered}
{ }^{4} \mathcal{P}_{n}^{I}: \sigma^{a}={ }^{4} \mathcal{P}_{n}^{I}: \sigma^{\mathcal{C}}={ }^{4} \mathcal{P}_{n}^{I}: \sigma^{I}, \\
{ }^{4} \mathcal{P}_{x}^{I}: \boldsymbol{\epsilon}^{a}={ }^{4} \mathcal{P}_{x}^{I}: \boldsymbol{\epsilon}^{\mathcal{c}}={ }^{4} \mathcal{P}_{x}^{I}: \boldsymbol{\epsilon}^{I} .
\end{gathered}
$$

Assuming the interface coordinate system, $\left\{\vec{e}_{1}^{I}, \vec{e}_{2}^{I}, \vec{n}^{I}\right\}$, where $\vec{n}^{I}$ denotes the unit normal vector of the interface, every second order tensor $A$, can be decomposed as $A=A_{x}+A_{n}$, with:

$$
A_{x}={ }^{4} \mathcal{P}_{x}^{I}: A=A_{11} \vec{e}_{1}^{I} \otimes \vec{e}_{1}^{I}+A_{22} \vec{e}_{2}^{I} \otimes \vec{e}_{2}^{I}+A_{12} \vec{e}_{1}^{I} \otimes \vec{e}_{2}^{I}+A_{21} \vec{e}_{2}^{I} \otimes \vec{e}_{1}^{I} \text {, (2.A.6) }
$$

$A_{n}={ }^{4} \mathcal{P}_{n}^{I}: A=A_{33} \vec{n}^{I} \otimes \vec{n}^{I}+A_{13} \vec{e}_{1}^{I} \otimes \vec{n}^{I}+A_{23} \vec{e}_{2}^{I} \otimes \vec{n}^{I}+A_{31} \vec{n}^{I} \otimes \vec{e}_{1}^{I}+A_{32} \vec{n}^{I} \otimes \vec{e}_{2}^{I}$.

Then, the constitutive behavior of each phase $(\pi=a, c)$ can be rewritten as:

$$
\begin{aligned}
& \sigma_{x}^{\pi}={ }^{4} \mathcal{C}_{x x}^{\pi}: \epsilon_{x}^{I}+{ }^{4} \mathcal{C}_{x n}^{\pi}: \epsilon_{n}^{\pi}, \\
& \sigma_{n}^{\pi}=\sigma_{n}^{I}={ }^{4} \mathcal{C}_{n x}^{\pi}: \epsilon_{x}^{I}+{ }^{4} \mathcal{C}_{n n}^{\pi}: \boldsymbol{\epsilon}_{n}^{\pi},
\end{aligned}
$$


where the approperiate components of phase stiffness tensor are indicated by the subscripts $x$ and $n$. The interface condition $\sigma_{n}^{a}=\sigma_{n}^{c}$, can then be rewritten as follows:

$$
{ }^{4} \mathcal{C}_{n x}^{a}: \boldsymbol{\epsilon}_{x}^{I}+{ }^{4} \mathcal{C}_{n n}^{a}: \boldsymbol{\epsilon}_{n}^{a}={ }^{4} \mathcal{C}_{n x}^{c}: \boldsymbol{\epsilon}_{x}^{I}+{ }^{4} \mathcal{C}_{n n}^{c}: \boldsymbol{\epsilon}_{n}^{c} .
$$

Substituting consistency condition (2.A.3) into (2.A.10) and some manipulation, leads to:

$$
\left(1-f^{c}\right)\left({ }^{4} \mathcal{C}_{n x}^{a}-{ }^{4} \mathcal{C}_{n x}^{c}\right): \boldsymbol{\epsilon}_{x}^{I}+{ }^{4} \mathcal{C}_{n n}^{a}: \boldsymbol{\epsilon}_{n}^{I}=\left[\left(1-f^{c}\right)^{4} \mathcal{C}_{n n}^{c}+f^{c 4} \mathcal{C}_{n n}^{a}\right]: \boldsymbol{\epsilon}_{n}^{c}
$$

which can be written as:

$$
\boldsymbol{\epsilon}_{n}^{c}={ }^{4} \mathcal{G}_{n}^{c}: \epsilon^{I},
$$

where the phase concentration tensor ${ }^{4} \mathcal{G}_{n}^{c}$ is given by:

$$
{ }^{4} \mathcal{G}_{n}^{c}=\left[\left(1-f^{c}\right)^{4} \mathcal{C}_{n n}^{c}+f^{c 4} \mathcal{C}_{n n}^{a}\right]^{-1}:\left[\left(1-f^{c}\right)\left({ }^{4} \mathcal{C}_{n x}^{a}-{ }^{4} \mathcal{C}_{n x}^{c}\right)+{ }^{4} \mathcal{C}_{n n}^{a}\right]
$$

Using again the consistency condition (2.A.3), leads to:

$$
\boldsymbol{\epsilon}_{n}^{a}={ }^{4} \mathcal{G}_{n}^{a}: \epsilon^{I},
$$

with

$$
{ }^{4} \mathcal{G}_{n}^{a}=\frac{1}{1-f^{c}}\left({ }^{4} \mathcal{P}_{n}-f_{c}^{4} \mathcal{G}_{n}^{c}\right)
$$

Then:

$$
\boldsymbol{\epsilon}^{\pi}=\boldsymbol{\epsilon}_{x}^{\pi}+\boldsymbol{\epsilon}_{n}^{\pi}=\left({ }^{4} \mathcal{P}_{x}+{ }^{4} \mathcal{G}_{n}^{\pi}\right): \boldsymbol{\epsilon}^{I}={ }^{4} \mathcal{H}^{\pi}: \boldsymbol{\epsilon}^{I}, \text { with } \quad \pi=a, c,
$$

with:

$$
{ }^{4} \mathcal{H}^{\pi}={ }^{4} \mathcal{P}_{x}+{ }^{4} \mathcal{G}_{n}^{\pi}, \quad \text { with } \quad \pi=a, c,
$$

and the constitutive behavior of each phase can consequently be rewritten as:

$$
\sigma^{\pi}={ }^{4} \mathcal{C}^{\pi}: \epsilon^{\pi}={ }^{4} \mathcal{C}^{\pi}:{ }^{4} \mathcal{H}^{\pi}: \boldsymbol{\epsilon}^{I}, \quad \text { with } \quad \pi=a, c .
$$

Finally, by using consistency equation (2.A.2) and equation (2.A.18), the elastic behavior of the composite can be written as:

$$
\sigma^{I}=\left[f^{c 4} \mathcal{C}^{c}:{ }^{4} \mathcal{H}^{c}+\left(1-f^{c}\right){ }^{4} \mathcal{C}^{a}:{ }^{4} \mathcal{H}^{a}\right]: \epsilon^{I}={ }^{4} \mathcal{C}^{I}: \epsilon^{I},
$$


which leads to

$$
{ }^{4} \mathcal{C}^{I}=\left[f^{c 4} \mathcal{C}^{c}:{ }^{4} \mathcal{H}^{c}+\left(1-f^{c}\right){ }^{4} \mathcal{C}^{a}:{ }^{4} \mathcal{H}^{a}\right]
$$




\section{B Appendix: Stiffness tensor of an elastic three-phase composite inclusion}

In this appendix, a closed form solution for the average elastic properties of a three-phase composite inclusion with orientation $\vec{n}^{I}$ is presented. The constitutive behavior of each phase is written as:

$$
\begin{aligned}
& \sigma_{x}^{\pi}={ }^{4} \mathcal{C}_{x x}^{\pi}: \epsilon_{x}^{I}+{ }^{4} \mathcal{C}_{x n}^{\pi}: \epsilon_{n}^{\pi} \quad ; \quad \pi=a, r, c, \\
& \sigma_{n}^{\pi}=\sigma_{n}^{I}={ }^{4} \mathcal{C}_{n x}^{\pi}: \epsilon_{x}^{I}+{ }^{4} \mathcal{C}_{n n}^{\pi}: \epsilon_{n}^{\pi} \quad ; \quad \pi=a, r, c,
\end{aligned}
$$

where ${ }^{4} \mathcal{C}^{\pi}$ is the fourth order elasticity tensor of phase $\pi$. The consistency conditions are given by:

$$
\begin{aligned}
& \sigma^{I}=f^{c} \sigma^{c}+f^{r} \sigma^{r}+f^{a} \sigma^{a}, \\
& \epsilon^{I}=f^{c} \epsilon^{c}+f^{r} \epsilon^{r}+f^{a} \epsilon^{a},
\end{aligned}
$$

and the interface conditions can be written as:

$$
\begin{gathered}
\boldsymbol{\sigma}_{n}^{a}=\boldsymbol{\sigma}_{n}^{r}=\boldsymbol{\sigma}_{n}^{c}=\sigma_{n}^{I}, \\
\boldsymbol{\epsilon}_{n}^{a}=\boldsymbol{\epsilon}_{n}^{r}=\boldsymbol{\epsilon}_{n}^{c}=\boldsymbol{\epsilon}_{n}^{I} .
\end{gathered}
$$

Using the interface condition $\sigma_{n}^{a}=\sigma_{n}^{r}=\sigma_{n}^{c}$, leads to:

$$
\begin{gathered}
\left({ }^{4} \mathcal{C}_{n x}^{a}-{ }^{4} \mathcal{C}_{n x}^{r}\right): \boldsymbol{\epsilon}_{x}^{I}={ }^{4} \mathcal{C}_{n n}^{r}: \boldsymbol{\epsilon}_{n}^{r}-{ }^{4} \mathcal{C}_{n n}^{a}: \boldsymbol{\epsilon}_{n}^{a}, \\
\left({ }^{4} \mathcal{C}_{n x}^{a}-{ }^{4} \mathcal{C}_{n x}^{c}\right): \boldsymbol{\epsilon}_{x}^{I}={ }^{4} \mathcal{C}_{n n}^{c}: \boldsymbol{\epsilon}_{n}^{c}-{ }^{4} \mathcal{C}_{n n}^{a}: \boldsymbol{\epsilon}_{n}^{a} .
\end{gathered}
$$

Combination of interface conditions (2.B.7) and (2.B.8), and substitution of equation (2.B.4), results, after some manipulation, in:

$$
\begin{aligned}
& {\left[f^{r}\left({ }^{4} \mathcal{C}_{n x}^{r}-{ }^{4} \mathcal{C}_{n x}^{a}\right)+\left(f^{a 4} \mathcal{C}_{n n}^{r}+f^{r 4} \mathcal{C}_{n n}^{a}\right):{ }^{4} \mathcal{C}_{n n}^{a^{-1}}:\left({ }^{4} \mathcal{C}_{n x}^{a}-{ }^{4} \mathcal{C}_{n x}^{c}\right)\right]: \boldsymbol{\epsilon}_{x}^{I}} \\
& +{ }^{4} \mathcal{C}_{n n}^{r}: \boldsymbol{\epsilon}_{n}^{I}=\left[f^{c 4} \mathcal{C}_{n n}^{r}+\left(f^{a 4} \mathcal{C}_{n n}^{r}+f^{r 4} \mathcal{C}_{n n}^{a}\right):{ }^{4} \mathcal{C}_{n n}^{a^{-1}}:{ }^{4} \mathcal{C}_{n n}^{c}\right]: \boldsymbol{\epsilon}_{n}^{c}
\end{aligned}
$$

which can be written as:

$$
\boldsymbol{\epsilon}_{n}^{c}={ }^{4} \mathcal{G}_{n}^{c}: \epsilon^{I},
$$


where the phase concentration tensor ${ }^{4} \mathcal{G}_{n}^{c}$ is given by:

$$
\begin{aligned}
{ }^{4} \mathcal{G}_{n}^{c} & =\left[f^{c 4} \mathcal{C}_{n n}^{r}+\left(f^{a 4} \mathcal{C}_{n n}^{r}+f^{r 4} \mathcal{C}_{n n}^{a}\right):{ }^{4} \mathcal{C}_{n n}^{a^{-1}}:{ }^{4} \mathcal{C}_{n n}^{c}\right]^{-1}:\left[f^{r}\left({ }^{4} \mathcal{C}_{n x}^{r}-{ }^{4} \mathcal{C}_{n x}^{a}\right)\right. \\
& \left.+\left(f^{a}{ }^{a} \mathcal{C}_{n n}^{r}+f^{r 4} \mathcal{C}_{n n}^{a}\right):{ }^{4} \mathcal{C}_{n n}^{a^{-1}}:\left({ }^{4} \mathcal{C}_{n x}^{a}-{ }^{4} \mathcal{C}_{n x}^{c}\right)+{ }^{4} \mathcal{C}_{n n}^{r}\right]
\end{aligned}
$$

Using the interface condition (2.B.8) leads to

$$
\epsilon_{n}^{a}={ }^{4} \mathcal{G}_{n}^{a}: \epsilon^{I}
$$

with

$$
{ }^{4} \mathcal{G}_{n}^{a}={ }^{4} \mathcal{C}_{n n}^{a^{-1}}:\left[{ }^{4} \mathcal{C}_{n n}^{c}:{ }^{4} \mathcal{G}_{n}^{c}-\left({ }^{4} \mathcal{C}_{n x}^{a}-{ }^{4} \mathcal{C}_{n x}^{c}\right):{ }^{4} \mathcal{P}_{x}\right]
$$

Using again the consistency condition (2.B.4) gives:

$$
\epsilon_{n}^{r}={ }^{4} \mathcal{G}_{n}^{r}: \epsilon^{I}
$$

with

$$
{ }^{4} \mathcal{G}_{n}^{r}=\frac{1}{f^{r}}\left({ }^{4} \mathcal{P}_{n}-f^{c 4} \mathcal{G}_{n}^{c}-f^{a 4} \mathcal{G}_{n}^{a}\right) .
$$

Then:

$$
\boldsymbol{\epsilon}^{\pi}=\boldsymbol{\epsilon}_{x}^{\pi}+\boldsymbol{\epsilon}_{n}^{\pi}=\left({ }^{4} \mathcal{P}_{x}+{ }^{4} \mathcal{G}_{n}^{\pi}\right): \boldsymbol{\epsilon}^{I}={ }^{4} \mathcal{H}^{\pi}: \boldsymbol{\epsilon}^{I}, \text { with } \pi=a, r, c,
$$

with:

$$
{ }^{4} \mathcal{H}^{\pi}={ }^{4} \mathcal{P}_{x}+{ }^{4} \mathcal{G}_{n}^{\pi}, \quad \text { with } \quad \pi=a, r, c .
$$

The constitutive behavior of each phase can consequently be rewritten as:

$$
\sigma^{\pi}={ }^{4} \mathcal{C}^{\pi}: \epsilon^{\pi}={ }^{4} \mathcal{C}^{\pi}:{ }^{4} \mathcal{H}^{\pi}: \boldsymbol{\epsilon}^{I}, \quad \text { with } \quad \pi=a, r, c .
$$

Finally, by using consistency equation (2.B.3) and equation (2.B.18), the elastic behavior of the three-phase composite inclusion can be written as:

$$
\sigma^{I}=\left[f^{c 4} \mathcal{C}^{c}:{ }^{4} \mathcal{H}^{c}+f^{r 4} \mathcal{C}^{r}:{ }^{4} \mathcal{H}^{r}+f^{a 4} \mathcal{C}^{a}:{ }^{4} \mathcal{H}^{a}\right]: \epsilon^{I},
$$


which leads to the stiffness tensor of an elastic three-phase composite inclusion in the form of:

$$
{ }^{4} \mathcal{C}^{I}=\left[f^{c 4} \mathcal{C}^{c}:{ }^{4} \mathcal{H}^{c}+f^{r 4} \mathcal{C}^{r}:{ }^{4} \mathcal{H}^{r}+f^{a 4} \mathcal{C}^{a}:{ }^{4} \mathcal{H}^{a}\right]
$$




\section{C Appendix: $\hat{e}$-inclusion model}

For the $\hat{\boldsymbol{\epsilon}}$-inclusion aggregate model, the local-global equilibrium and compatibility conditions are given by:

$$
\begin{aligned}
{ }^{4} \mathcal{P}_{x}^{I^{i}}: \sigma^{I^{i}}={ }^{4} \mathcal{P}_{x}^{I^{i}}: \bar{\sigma} ; & i=1, \ldots, N, \\
{ }^{4} \mathcal{P}_{n}^{I^{i}}: \boldsymbol{\epsilon}^{I^{i}}={ }^{4} \mathcal{P}_{n}^{I^{i}}: \hat{\boldsymbol{\epsilon}} ; & i=1, \ldots, N .
\end{aligned}
$$

For a system consisting of $N$ inclusions, for a macroscopic stress $\bar{\sigma}, 3 N$ local unknowns $\left(\sigma_{n}^{I^{i}}={ }^{4} \mathcal{P}_{n}^{I^{i}}: \sigma^{I^{i}}\right)$ and 6 global unknowns $\hat{\boldsymbol{\epsilon}}$, must be obtained to determine the state of each composite inclusion from the following equations:

$$
\begin{aligned}
& { }^{4} \mathcal{P}_{n}^{I^{i}}: \boldsymbol{\epsilon}^{I^{i}}\left(\sigma^{I^{i}}\right)={ }^{4} \mathcal{P}_{n}^{I^{i}}: \hat{\boldsymbol{\epsilon}} ; \quad i=1, \ldots, N, \\
& \bar{\sigma}=\left\langle\sigma^{I^{i}}\right\rangle=\left\langle{ }^{4} \mathcal{P}_{x}^{I^{i}}: \bar{\sigma}+\sigma_{n}^{I^{i}}\right\rangle .
\end{aligned}
$$

To obtain the macroscopic stiffness tensor, equations (2.C.3) and (2.C.4) are rewritten as:

$$
\begin{aligned}
& \boldsymbol{\Psi}_{n}^{i}\left(\sigma_{n}^{I^{i}}, \hat{\boldsymbol{\epsilon}}, \overline{\boldsymbol{\sigma}}\right)=\boldsymbol{O}_{n}^{i} ; \quad i=1, \ldots, N, \\
& \boldsymbol{\Psi}_{\sigma}\left(\sigma_{n}^{I^{i}}, \overline{\boldsymbol{\sigma}}\right)=\boldsymbol{O} .
\end{aligned}
$$

For any variation of the macroscopic stress $\bar{\sigma}$, the equations remain $O$, so that:

$$
\begin{aligned}
& \frac{d \Psi_{n}^{i}}{d \overline{\boldsymbol{\sigma}}}=\frac{\partial \Psi_{n}^{i}}{\partial \overline{\boldsymbol{\sigma}}}+\frac{\partial \Psi_{n}^{i}}{\partial \sigma_{n}^{I^{i}}}: \frac{d \boldsymbol{\sigma}_{n}^{I^{i}}}{d \overline{\boldsymbol{\sigma}}}+\frac{\partial \boldsymbol{\Psi}_{n}^{i}}{\partial \hat{\boldsymbol{\epsilon}}}: \frac{d \hat{\boldsymbol{\epsilon}}}{d \overline{\boldsymbol{\sigma}}}={ }^{4} \boldsymbol{O}_{n}^{i} ; \quad i=1, \ldots, N, \\
& \frac{d \Psi_{\sigma}}{d \overline{\boldsymbol{\sigma}}}=\frac{\partial \boldsymbol{\Psi}_{\sigma}}{\partial \overline{\boldsymbol{\sigma}}}+\sum_{j=1}^{n} \frac{\partial \Psi_{\sigma}}{\partial \sigma_{n}^{I^{j}}}: \frac{d \boldsymbol{\sigma}_{n}^{I^{j}}}{d \overline{\boldsymbol{\sigma}}}={ }^{4} \boldsymbol{O},
\end{aligned}
$$

from which, $\frac{d \hat{\epsilon}}{d \bar{\sigma}}$ and $\frac{d \sigma_{n}^{i}}{d \bar{\sigma}}$ can be determined:

$$
\frac{d \hat{\boldsymbol{\epsilon}}}{d \overline{\boldsymbol{\sigma}}}=\left[\sum_{j=1}^{n} \frac{\partial \boldsymbol{\Psi}_{\sigma}}{\partial \sigma_{n}^{I^{j}}}:\left(\frac{\partial \boldsymbol{\Psi}_{n}^{j}}{\partial \sigma_{n}^{I^{j}}}\right)^{-1}: \frac{\partial \boldsymbol{\Psi}_{n}^{j}}{\partial \hat{\boldsymbol{\epsilon}}}\right]^{-1}:\left[\frac{\partial \boldsymbol{\Psi}_{\sigma}}{\partial \bar{\sigma}}-\sum_{j=1}^{n} \frac{\partial \boldsymbol{\Psi}_{\sigma}}{\partial \sigma_{n}^{I^{j}}}:\left(\frac{\partial \Psi_{n}^{j}}{\partial \sigma_{n}^{I^{j}}}\right)^{-1}: \frac{\partial \boldsymbol{\Psi}_{n}^{j}}{\partial \bar{\sigma}}\right],
$$


and

$$
\begin{aligned}
\frac{d \sigma_{n}^{I^{i}}}{d \overline{\boldsymbol{\sigma}}}=\left(\frac{\partial \Psi_{n}^{i}}{\partial \sigma_{n}^{I^{i}}}\right)^{-1}:\{ & -\frac{\partial \Psi_{n}^{i}}{\partial \bar{\sigma}}-\frac{\partial \Psi_{n}^{i}}{\partial \hat{\boldsymbol{\epsilon}}}:\left[\sum_{j=1}^{n} \frac{\partial \Psi_{\sigma}}{\partial \sigma_{n}^{I^{j}}}:\left(\frac{\partial \Psi_{n}^{j}}{\partial \sigma_{n}^{I^{j}}}\right)^{-1}: \frac{\partial \Psi_{n}^{j}}{\partial \hat{\boldsymbol{\epsilon}}}\right]^{-1}: \\
& {\left.\left[\frac{\partial \Psi_{\sigma}}{\partial \bar{\sigma}}-\sum_{j=1}^{n} \frac{\partial \Psi_{\sigma}}{\partial \sigma_{n}^{I^{j}}}:\left(\frac{\partial \Psi_{n}^{j}}{\partial \sigma_{n}^{I^{j}}}\right)^{-1}: \frac{\partial \Psi_{n}^{j}}{\partial \bar{\sigma}}\right]\right\} }
\end{aligned}
$$

Note that the derivatives of $\Psi_{n}^{i}$ and $\Psi_{\sigma}$ can be straightforwardly determined and depend on the stiffnesses of the composite inclusions and the orientations of their internal interfaces. The fourth-order macroscopic compliance tensor, and consequently the stiffness tensor, can then be obtained as:

$$
\begin{aligned}
& \overline{\boldsymbol{\epsilon}}=\left\langle\boldsymbol{\epsilon}^{I^{i}}\left(\sigma_{n}^{I^{i}}, \bar{\sigma}\right)\right\rangle, \\
& { }^{4} \mathcal{S}=\frac{d \bar{\epsilon}}{d \bar{\sigma}}=\left\langle\frac{\partial \epsilon^{I^{i}}}{\partial \bar{\sigma}}+\frac{\partial \epsilon^{I^{i}}}{\partial \sigma_{n}^{I^{i}}}: \frac{d \sigma_{n}^{I^{i}}}{d \bar{\sigma}}\right\rangle, \\
& { }^{4} \mathcal{C}={ }^{4} \mathcal{S}^{-1}=\left\langle\frac{\partial \epsilon^{I^{i}}}{\partial \bar{\sigma}}+\frac{\partial \epsilon^{I^{i}}}{\partial \sigma_{n}^{I^{i}}}: \frac{d \sigma_{n}^{I^{i}}}{d \bar{\sigma}}\right\rangle^{-1},
\end{aligned}
$$

where also the derivatives of $\epsilon^{I^{i}}$ are known. 


\section{References}

[1] X. Guan and R. Pitchumani. A micromechanical model for the elastic properties of semicrystalline thermoplastic polymers. Polymer Engineering and Science 44:433-451, 2004.

[2] P.D. Davidse, H.I. Waterman, and J.B. Westerdijk. Sound velocity and Young's modulus in polyethylene. Journal of Polymer Science 59:389-400, 1962.

[3] B. Crist, C.J. Fisher, and P.R. Howard. Mechanical properties of model polyethylenes: tensile elastic modulus and yield stress. Macromolecules 22:1709-1718, 1989.

[4] J. Janzen. Elastic moduli of semicrystalline polyethylenes compared with theoretical micromechanical models for composites. Polymer Engineering and Science 32:1242-1254, 1992.

[5] F. Bedoui, J. Diani, G. Regnier, and W. Seiler. Micromechanical modeling of isotropic elastic behavior of semicrystalline polymers. Acta Materialia 54:1513-1523, 2006.

[6] B.J. Lee, D.M. Parks, and S. Ahzi. Micromechanical modeling of large plastic deformation and texture evolution in semicrystalline polymers. Journal of the Mechanics and Physics of Solids 41:1651-1687, 1993.

[7] B.J. Lee, A.S. Argon, D.M. Parks, S. Ahzi, and Z. Bartczak. Simulation of large strain plastic deformation and texture evolution in high density polyethylene. Polymer 34:3555-3575, 1993.

[8] B.J. Lee, S. Ahzi, and R.J. Asaro. On the plasticity of low symmetry crystals lacking 5 independent slip systems. Mechanics of Materials 20:1-8, 1995.

[9] S. Nikolov and I. Doghri. A micro/macro constitutive model for the small-deformation behavior of polyethylene. Polymer 41:1883-1891, 2000.

[10] S. Nikolov, I. Doghri, O. Pierard, L. Zealouk, and A. Goldberg. Multi-scale constitutive modeling of the small deformations of semi-crystalline polymers. Journal of the Mechanics and Physics of Solids 50:2275-2302, 2002.

[11] F. Bedoui, J. Diani, and G. Regnier. Micromechanical modeling of elastic properties in polyolefins. Polymer 45:2433-2442, 2004.

[12] J.A.W. van Dommelen, D.M. Parks, M.C. Boyce, W.A.M Brekelmans, and F.P.T Baaijens. Micromechanical modeling of the elasto-viscoplastic behavior of semi-crystalline polymers. Journal of the Mechanics and Physics of Solids 51:519-541, 2003.

[13] J.C. Halpin and J.L. Kardos. Moduli of crystalline polymers employing composite theory. Journal of Applied Physics 43:2235-2241, 1972.

[14] P.J. Phillips and J. Patel. The influence of morphology on the tensile properties of polyethylenes. Polymer Engineering and Science 18:943-950, 1978.

[15] T.N. Pham and C.L. Tucker. The interlamellar phase controls stiffness in semicrystalline polymers. In: 24th Annual Meeting of the Polymer Processing Society, PPS-24. Salerno, Italy, 2008.

[16] T. Mori and K. Tanaka. Average stress in matrix and average elastic energy of materials with misfitting inclusions. Acta Metallurgica 21, 1973.

[17] L. Lin and A.S. Argon. Structure and plastic deformation of polyethylene. Journal of Materials Science 29:294-323, 1994. 
[18] O. Gueguen, S. Ahzi, A. Makradi, and S. Belouettar. A new three-phase model to estimate the effective elastic properties of semi-crystalline polymers: Application to PET. Mechanics of Materials 42:1-10, 2010.

[19] E.W. Fischer and G.F. Schmidt. Über der Langperioden von verstrectem Polyathylen. Angewandte Chemie 74:551-562, 1962.

[20] B.A.G. Schrauwen, R.P.M. Janssen, L.E. Govaert, and H.E.H. Meijer. Intrinsic deformation behavior of semicrystalline polymers. Macromolecules 37:6069-6078, 2004.

[21] H.P. Klug and L.E. Alexander. X-ray Diffraction Procedures: For Polycrystalline And Amorphous Materials, 2nd Edition. Wiley-Interscience, 1974.

[22] D.W. van Krevelen. Properties of polymers. Elsevier, 1990.

[23] D.C. Basset and A.M. Hodge. On the morphology of melt-crystallized polyethylene I. Lamellar profiles. Proceedings of the Royal Society of London A 377:25-37, 1981.

[24] C. G'Sell and A. Dahoun. Evolution of microstructure in semi-crystalline polymers under large plastic deformation. Materials Science and Engineering A 175:183-199, 1994.

[25] P.J. Veld, M. Hutter, and G.C. Rutledge. Temperature-dependent thermal and elastic properties of the interlamellar phase of semicrystalline polyethylene by molecular simulation. Macromolecules 39:439-447, 2006.

[26] R.G. Strobl and W. Hagedorn. Raman spectroscopic method for determining the crystallinity of polyethylene. Journal of Polymer Science: Polymer Physics Edition 16:11811193, 1978.

[27] W. Lin, M. Cossar, V. Dang, and J. Tech. The application of Raman spectroscopy to threephase characterization of polyethylene crystallinity. Polymer Testing 26:814-821, 2007.

[28] F. Daver and B.W. Cherry. Effect on the elastic modulus of high-density polyethylene of differing thermal treatments. Journal of Applied Polymer Science 58:2429-2432, 1995.

[29] A. Galeski. Strength and toughness of crystalline polymer systems. Progress in Polymer Science 28:1643-1699, 2003.

[30] S. Gautam, S. Balijepalli, and G.C. Rutledge. Molecular simulations of the interlamellar phase in polymers: effect of chain tilt. Macromolecules 33:9136-9145, 2000.

[31] L.E. Govaert, B. Brown, and P. Smith. Temperature dependence of the Young's modulus of oriented polyethylene. Macromolecules 25:3480-3483, 1992.

[32] T.L. Phillips and S. Hanna. A comparison of computer models for the simulation of crystalline polyethylene and the long n-alkanes. Polymer 46:11003-11018, 2005.

[33] A. Odajima and T. Maeda. Calculation of the elastic constants and the lattice energy of the polyethylene crystal. Journal of Polymer Science Part C: Polymer Symposia 15:55-74, 1967.

[34] N. Karasawa, S. Dasgupta, and W.A. Goddard III. Mechanical properties and force field parameters for polyethylene crystal. Journal of Physical Chemistry 95:2260-2272, 1991.

[35] D.J. Lacks and G.C. Rutledge. Simulation of the temperature dependence of mechanical properties of polyethylene. Journal of Physical Chemistry 98:1222-1231, 1994.

[36] K. Tashiro, M. Kobayashi, and H. Tadakoro. Calculation of the three-dimensional elastic constants of polymer crystals. 2. Application to orthorhombic polyethylene and poly(vinyl alcohol). Macromolecules 11:914-918, 1978. 
[37] C.L. Choy and W.P. Leung. Elastic moduli of ultradrawn polyethylene. Journal of Polymer Science: Polymer Physics Edition 23:1759-1780, 1985.

[38] D.W. Hadley and I.M. Ward. Structure and properties of oriented polymers, second edition. Chapman \& Hall, 1997.

[39] R.J. Young and P.A. Lovell. Introduction to polymers, Second edition. Chapman \& Hall, 1991.

[40] K. Tashiro and M. Kobayashi. Molecular theoretical study of the intimate relationships between structure and mechanical properties of polymer crystals. Polymer 37:1775-1786, 1996.

[41] W.W. Graessley and S.F. Edwards. Entanglement interactions in polymers and the chain contour concentration. Polymer 22:1329-1334, 1981.

[42] S. Wu. Chain structure and entanglement. Journal of Polymer Science Part B: Polymer Physics 27:723-741, 1989.

[43] C. Liu, J. He, E. van Ruymbeke, R. Keunings, and C. Bailly. Evaluation of different methods for the determination of the plateau modulus and the entanglement molecular weight. Polymer 47:4461-4479, 2006.

[44] J.M. Dealy and R.G. Larson. Structure and rheology of molten polymers. Hanser, 2006. 


\title{
Deformation kinetics and texture evolution 1
}

Chapter 3

\begin{abstract}
The mechanical behavior of semicrystalline polymers is strongly dependent on their crystallinity level, the initial underlying microstructure, and the evolution of this structure during deformation. A previously developed micromechanical constitutive model is used to capture the elasto-viscoplastic deformation and texture evolution in semicrystalline polymers. The model represents the material as an aggregate of twophase layered composite inclusions, consisting of crystalline lamellae and amorphous layers. This work focuses on adding quantitative abilities to the multi-scale constitutive model, in particular for the stress-dependence of the rate of plastic deformation, referred to as the slip kinetics. In order to do that, the previously used viscoplastic power law relation is replaced with an Eyring flow rule. The slip kinetics are then re-evaluated and characterized using a hybrid numerical/experimental procedure, and the results are validated for uniaxial compression data of HDPE, at various strain rates. A double yield phenomenon is observed in the model prediction. Texture analysis shows that the double yield point in the model is due to morphological changes during deformation, that induce a change of deformation mechanism.
\end{abstract}

\footnotetext{
${ }^{1}$ Reproduced from: A. Sedighiamiri, L.E. Govaert, J.A.W. van Dommelen, Micromechanical Modeling of the Deformation Kinetics of Semicrystalline Polymers, Journal of Polymer Science Part B: Polymer Physics, 49: 1297-1310, 2011.
} 


\subsection{Introduction}

The process of plastic deformation in semicrystalline polymers is complicated due to the operation of a variety of mechanisms at different levels and is strongly dependent on the underlying microstructure of these materials. Any macroscopically homogeneous deformation is accommodated by various deformation mechanisms in the heterogeneous microstructure. Mechanical properties and morphological changes of semicrystalline polymers, particularly polyethylene, during plastic deformation have been studied extensively by experiments [1-13]. It is well established that the principal mechanisms involved in the plastic deformation of polymer crystals, are crystallographic in nature. During the early and moderate stages of deformation, the crystals become distorted but the crystalline lamellae remain undestroyed, whereas at large strains, the original crystalline microstructure breaks down and a new fibrillar structure forms [14].

The elastic and viscoplastic behavior of semicrystalline polymers strongly depend on the degree of crystallinity and the initial underlying microstructure, as well as the evolution in this microstructure during deformation. There does not seem to be a model yet, which can explain the level of yield stress in terms of the degree of crystallinity, morphological characteristics and strain rate in these materials. Popli and Mandelkern [15] studied the influence of structural and morphological factors on the mechanical properties of polyethylene. They reported that the yield stress was a linear function of the degree of crystallinity and the crystallite thickness, whereas the molecular weight had no direct influence on the yield stress.

A first attempt to directly relate the crystalline structure to the yield stress of semicrystalline PE was made by Young [16, 17], who regarded yield to be controlled by the nucleation of dislocations. His approach assumes that yield involves the thermal activation of chain screw dislocations within the crystalline lamellae, resulting in a direct relation between macroscopic yield stress and lamellar thickness. The method was further expanded by Séguéla et al. [18, 19] and Nikolov and Raabe [20], who introduced the driving force for the nucleation and propagation of chain screw dislocations to rely on $180^{\circ}$ chain twist defects that migrate along the chain stems, enabling them to further analyse the deformation kinetics (temperature and strain rate-dependence). Drawbacks of this type of modeling, however, are that it is only applicable to isotropic systems, assumes that crystallinity has no influence, and only accounts for a single crystallographic slip system, whereas it is well known that single PE lamellae have more modes of deformation. Lack of information about the evolution of texture is another disadvantage of Young's approach.

To enable the analysis of texture evolution during deformation Parks and 
Ahzi [21] included all physically available crystallographic slip systems in a polycrystalline approach. They proposed a viscoplastic Taylor-type model for a purely crystalline material lacking five independent slip systems, with which they were able to qualitatively predict texture development in HDPE during uniaxial tension and simple shear. However, using Taylor's assumption leads to a very high post-yield stress-strain behavior and overestimates the rate of the texture evolution. The approach was improved significantly by formulating more sophisticated interaction laws and by the introduction of the amorphous phase. Lee et al. [22-24] developed a specific micromechanical model in which a rigid-viscoplastic composite inclusion model, consisting of mechanically coupled amorphous and crystalline phases, was used in combination with a Sachs-like and two hybrid interaction laws to predict the stress-strain behavior and crystallographic texture evolution of HDPE. Van Dommelen et al. [25-27] have extended the rigid-viscoplastic approach of Lee et al. to an elasto-viscoplastic model for large deformation of semicrystalline polymers. In this model, the behavior of the amorphous phase was assumed to be isotropic elastic with a rate-dependent plastic flow with strain hardening resulting from molecular orientation. The crystalline phase was modeled as anisotropic elastic with plastic flow occurring via crystallographic slip. The concept of two-phase composite inclusions was used in combination with a self-consistent approach by Nikolov et al. [28]. Recently, Sedighiamiri et al. [29] developed a layered composite inclusion model in an elastic framework to quantitatively predict the elastic properties of polyethylene, where also the influence of the rigid-amorphous phase was considered.

The present work is directed towards adding the same quantitative predictive abilities to the previously developed multiscale, micromechanical model [25-27] for the prediction of the yield and post-yield behavior, and texture evolution in semicrystalline polymers during large deformations at different strain rates. Semicrystalline polyethylene is considered as a case study. A critical factor is the stress-dependence of the rate of plastic deformation, the slip kinetics, which is the mechanism underlying time-dependent, macroscopic failure. The kinetics of the macroscopic plastic flow strongly depends on the slip kinetics of the individual crystallographic slip systems. Therefore, an accurate quantitative prediction requires a proper description of the rate-dependence of slip along crystallographic planes. As a first step in achieving this goal, the previously used viscoplastic power law relation [25] is replaced with an Eyring flow rule [30, 31]. The re-evaluation of the slip kinetics is performed using a combined numerical/experimental approach and the refined slip kinetics are then validated for uniaxial compression data of HDPE, for different strain rates. It is also attempted to validate the prediction of texture evolution by comparing it to the 
limited experimental data, available in literature. A double yield phenomenon is found in the model prediction and is related to morphological changes that induce a change of deformation mechanism.

\subsection{Experimental}

The semicrystalline polymer used in this study is a HDPE grade supplied by Sabic (Stamylan HD 9089S), with a weight-average molecular weight of $\mathrm{M}_{\mathrm{w}}=$ $70,000[\mathrm{~g} / \mathrm{mol}]$ and a number-average molecular weight of $\mathrm{M}_{\mathrm{n}}=11,000[\mathrm{~g} / \mathrm{mol}]$. HDPE samples were compression molded between brass plates and aluminum foil at $200{ }^{\circ} \mathrm{C}$. The degree of crystallinity of samples was measured by wide angle $\mathrm{X}$-ray scattering experiments. Compression tests were performed at room temperature with varying strain rates.

\subsection{Model description}

The constitutive behavior of microscopically heterogeneous semicrystalline material is modeled by an aggregate of layered two-phase composite inclusions [22, 23]. Each composite inclusion is represented by a crystalline lamella and an amorphous layer. A microstructural elasto-viscoplastic constitutive model is used for both constituent phases. In this section, the kinematical framework and the constitutive models for the elastic and viscoplastic behavior of each phase together with the polycrystalline aggregate model are briefly discussed. The formulation of the material models and the sets of equations that comprise the composite inclusion model are summarized in Appendix 3.A. For a more detailed discussion of the models employed here, see Van Dommelen et al. [25]

\subsubsection{Kinematics}

For a microstructural elasto-viscoplastic constitutive description of the constituent phases, the deformation gradient tensor $\boldsymbol{F}=\left(\vec{\nabla}_{0} \vec{x}\right)^{T}$ of each phase is decomposed into a plastic and an elastic part, denoted by the subscripts "p" and "e", respectively [32]:

$$
\boldsymbol{F}^{\pi}=\boldsymbol{F}_{\mathrm{e}}^{\pi} \cdot \boldsymbol{F}_{\mathrm{p}}^{\pi}, \quad \text { with } \quad \pi=\mathrm{a}, \mathrm{c},
$$

where the superscript $\pi$ shows either the amorphous or the crystalline phase. Subsequently, the constitutive behavior of both the crystalline and the amorphous 
phase is specified.

\subsubsection{Crystalline phase}

The crystalline phase of semicrystalline polyethylene consists of regularly ordered chain segments in an orthorhombic packing. This crystal structure causes the crystalline phase to possess highly anisotropic elastic properties with a high elastic modulus in the chain direction, and to have plastic deformation limited to crystallographic slip at the initial stages of deformation [5, 8, 9, 33]. Moreover, polymer crystals may also deform plastically by mechanical twinning and stressinduced martensitic phase transformation [5, 33, 34]. Since crystallographic slip is the predominant deformation mode up to moderate strains, for the modeling purpose the latter two mechanisms are left out of consideration.

The elastic part of the deformation in the crystalline domain is characterized by an anisotropic fourth order elasticity tensor, which linearly relates the GreenLagrange strain tensor and the elastic second Piola-Kirchhoff stress measure.

For the viscoplastic behavior of the crystalline phase, a rate-dependent crystal plasticity model is used. In this model, the plastic velocity gradient of the crystalline lamella, consisting of a single crystal, is described by the contributions of all $N_{s}$ physically distinct slip systems, being 8 for high density polyethylene, and is given by:

$$
\boldsymbol{L}_{\mathrm{p}}^{\mathrm{c}}=\dot{\boldsymbol{F}}_{\mathrm{p}}^{\mathrm{c}} \cdot \boldsymbol{F}_{\mathrm{p}}^{\mathrm{c}-1}=\sum_{\alpha=1}^{N_{s}} \dot{\gamma}^{\alpha} \boldsymbol{P}_{0}^{\alpha} \quad ; \quad \boldsymbol{P}_{0}^{\alpha}=\vec{s}_{0}^{\alpha} \otimes \vec{n}_{0}^{\alpha},
$$

with $\boldsymbol{P}_{0}^{\alpha}$ the nonsymmetric Schmid tensor, defined by the dyadic product of the unit slip direction $\vec{s}_{0}^{\alpha}$ and the unit slip plane normal $\vec{n}_{0}^{\alpha}$ in the reference configuration. The shear rate of each slip system $\dot{\gamma}^{\alpha}$ was previously assumed to be related to the corresponding resolved shear stress $\tau^{\alpha}$ via the viscoplastic power law relation:

$$
\dot{\gamma}^{\alpha}=\dot{\gamma}_{0}^{c} \frac{\tau^{\alpha}}{g^{\alpha}}\left|\frac{\tau^{\alpha}}{g^{\alpha}}\right|^{n^{c}-1},
$$

with $g^{\alpha}$ the critical resolved shear stress of the $\alpha$ th slip system, $\dot{\gamma}_{0}^{c}$ a reference shear rate and $n^{\mathrm{c}}$ the rate exponent. Here, this relation is replaced with an Eyring flow rule:

$$
\dot{\gamma}^{\alpha}=\dot{\zeta}_{0}^{\alpha} \sinh \left[\frac{\tau^{\alpha}}{\tau_{0}^{\mathrm{c}}}\right],
$$


with $\dot{\zeta}_{0}^{\alpha}$ and $\tau_{0}^{\mathrm{c}}$ the reference shear rate and the shear strength, respectively. Eyring [30, 31] developed a theory to describe the relaxation process of viscous flow in liquids, and it can also be used to describe the yield behavior of amorphous polymers, as well as the microscopic plastic flow at the level of crystallographic slip systems in semicrystalline polymers. If the resolved shear stress is small, equation (3.4) predicts a linear relation between the resolved shear rate and the resolved shear stress of each slip system. For large stress, at which $\sinh \left[\frac{\tau^{\alpha}}{\tau_{0}^{\alpha}}\right] \approx \frac{1}{2} \exp \left[\frac{\tau^{\alpha}}{\tau_{0}^{\alpha}}\right]$, the resolved shear rate of each slip system increases exponentially with increasing resolved shear stress.

\subsubsection{Amorphous phase}

The amorphous domain of semicrystalline polyethylene consists of an assembly of randomly coiled macromolecules, constrained by the adjacent crystalline lamellae. In the case of polyethylene, the glass transition temperature of the amorphous phase is far below room temperature. The amorphous phase should, therefore, be in the rubbery state at room temperature. However, there are some indications in literature that suggest that the amorphous layers may be stiffer than purely bulk amorphous material due to the confinement of amorphous layers between thick crystalline lamellae [35]. Boyd [36] showed that for the amorphous phase, the relaxed modulus associated with the glass-rubber relaxation ( $\beta$ process) is very high due to the constraints by the neighboring lamellae, leading to the immobilization of amorphous segmental re-orientation.

Furthermore, random thermal fluctuations allow chain ends or loose chain folds in the amorphous phase to migrate into the crystalline lamellae. Chain segments can similarly escape from lamellae into the amorphous regions. This process of chain diffusion [37] is often referred to as $\alpha$-relaxation as well (see figure 3.1] for a schematic representation). Chain diffusion results in redistribution of the tight and loose chain folds, cilia or intercrystalline links that constitute the amorphous layer [36]. Therefore, relaxation of the interlamellar material occurs in a ratedependent process and its kinetics is affected by that of the crystalline phase. In line with that, also the amorphous phase is modeled as elasto-viscoplastic.

In earlier work [25], a Boyce-Parks-Argon-like model [38] combined with a viscoplastic power law relation was employed to model the elasto-viscoplastic deformation of the amorphous domain. Here, in the same framework, the effective shear strain rate and the effective shear stress are related by an Eyring flow rule. The plastic rate of stretching is defined by an associated flow rule [25]. The Arruda-Boyce eight-chain network rubber elasticity model [39] is used to account for orientation induced hardening [38, 40]. 


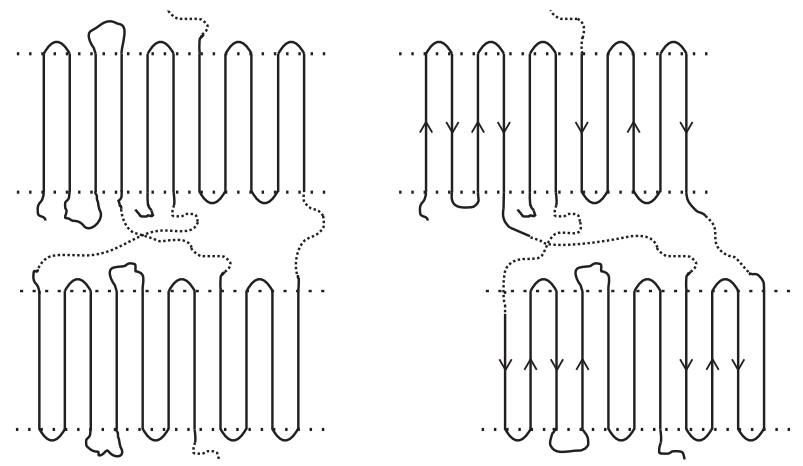

Figure 3.1 Schematic representation of chain diffusion in the lamellae of polyethylene.

\subsubsection{Composite inclusion model}

The mechanical behavior of the microscopically heterogeneous material is modeled by an aggregate of a number of layered two-phase composite inclusions, as proposed by Lee et al. [22, 23] for rigid-viscoplastic material behavior. Each composite inclusion consists of a crystalline lamella, which is mechanically coupled to its adjacent amorphous layer to form a lamellar morphology, characterized by crystallographic texture (lattice orientation) and morphological texture (interface orientation), as shown in figure 3.2. The stress

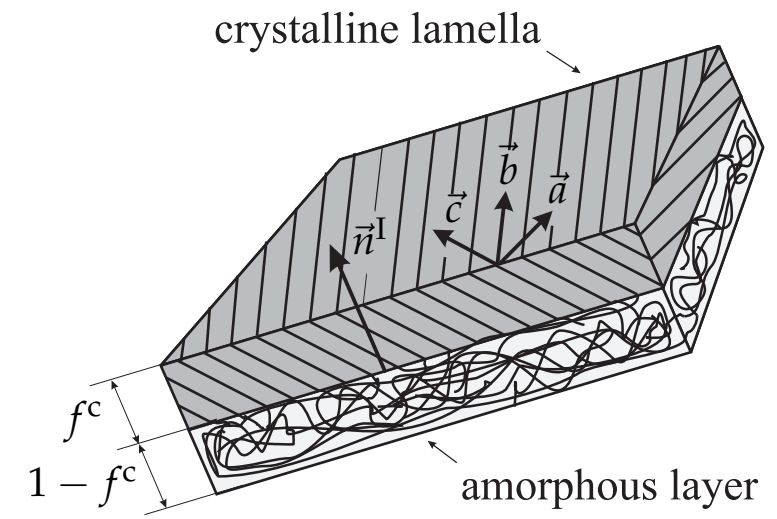

Figure 3.2 Schematic representation of a two-phase composite inclusion.

and deformation fields in each separate phase of the composite inclusion are assumed to be piecewise homogeneous; but, they can be different between the two coupled phases. The inclusion-averaged Cauchy stress and the inclusionaveraged deformation gradient are given by the volume-average of the fields of the constituent phases. Furthermore, the crystalline lamella and the amorphous 
layer are assumed to be kinematically compatible and in equilibrium across the interface.

To relate the volume-averaged mechanical behavior of each layered composite inclusion to the aggregate of composite inclusions, a hybrid local-global interaction law [22, 23, 25] is formulated. The consistency conditions for equilibrium and compatibility of the aggregate are maintained by enforcing the macroscopic stress and deformation to equal the volume-average of the stress and deformation fields of the composite inclusions.

In the hybrid interaction model, local-global compatibility conditions are assumed for the projections of the inclusion-averaged fields for which intrainclusion equilibrium conditions were formulated. Inversely, local-global equilibrium conditions are formulated for the components of the inclusion-averaged fields that are subjected to intra-inclusion compatibility. A more elaborate description of the composite inclusion model is presented in [25].

\subsubsection{Texture evolution}

Crystallographic slip within the crystalline lamellae can take place by either fine slip or coarse slip [33]. During the early stages of deformation, fine slip, which changes the angle between the chain direction and the interface normal, is the predominant mode of plastic deformation, whereas coarse slip, which does not alter the angle between chain and interface normal, becomes more important at larger strains. In this study, all slips are assumed to be of the fine slip type.

There are two types of texture development present in the model, namely, crystallographic texture which determines the orientation of the crystallographic axes within the crystalline lamellae, and morphological texture which is represented by the orientation of normal vectors to the crystalline-amorphous interface. These two kinds of texture can evolve independently during the plastic deformation process. Upon large plastic deformation, texture evolutions have a great influence on the stress-strain behavior of a semicrystalline polymer. For instance, a weak textural strain-hardening is observed in simple shear of HDPE, whereas in uniaxial tension, a strong strain-hardening due to texture evolution is found [41].

\subsubsection{Deformation modes}

Initially isotropic material is represented by an aggregate of randomly oriented composite inclusions. The mechanical behavior of these aggregates is investigated for two deformation modes, namely uniaxial tension and uniaxial 
compression. For this purpose, an aggregate of composite inclusions is subjected to a constant strain rate in the principal 1-direction:

$$
\overline{\boldsymbol{R}}=\boldsymbol{I} \quad ; \quad \bar{U}_{11}=\lambda(t),
$$

with

$$
\begin{aligned}
& \lambda(t)=\exp (\dot{\epsilon} t) \quad \text { for tension, } \\
& \lambda(t)=\exp (-\dot{\epsilon} t) \quad \text { for compression, }
\end{aligned}
$$

where $\bar{R}$ is the macroscopic rotation tensor, and $\bar{U}$ is the corresponding right stretch tensor. Moreover, the components of the macroscopic Cauchy stress tensor $\bar{\sigma}$ should satisfy:

$$
\bar{\sigma}_{22}=\bar{\sigma}_{33}=\bar{\sigma}_{12}=\bar{\sigma}_{13}=\bar{\sigma}_{23}=0 \text {. }
$$

\subsection{Initial model parameters and results}

In this section, the elasto-viscoplastic composite inclusion model is used to study the mechanical behavior of initially isotropic HDPE, using a power law relation for the kinetics of crystalline slip and plastic deformation of the amorphous phase with the same viscoplastic parameters as used in previous works [25-27].

\subsubsection{Crystalline phase}

The elastic behavior of the crystalline phase is anisotropic with a high elastic modulus in the chain direction. For orthorhombic PE crystals, the anisotropic fourth order elasticity tensor involves nine independent elastic constants. The constants used in this model are given in table 3.1 .

Table 3.1 Elastic constants [GPa] for the crystalline phase of PE [42].

\begin{tabular}{ccccccccc}
\hline$C_{11}$ & $C_{22}$ & $C_{33}$ & $C_{12}$ & $C_{13}$ & $C_{23}$ & $C_{44}$ & $C_{55}$ & $C_{66}$ \\
\hline 7.99 & 9.92 & 315.92 & 3.28 & 1.13 & 2.14 & 3.62 & 1.62 & 3.19 \\
\hline
\end{tabular}

There are eight physically distinct slip systems present in the crystalline domain of HDPE, which effectively comprise four independent deformation modes. 
When the direction of slip is parallel to the molecular chain direction, the deformation mechanism is called chain slip, whereas in case the slip direction is perpendicular to the chain direction, the slip mechanism is referred to as a transverse slip system. Table 3.2 summarizes all distinct slip systems together with their critical resolved shear stresses $g^{\alpha}$ in the viscoplastic power law relation at room temperature, normalized to the critical resolved shear stress of the easiest slip system $g_{0}=g^{(100)[001]}=8 \mathrm{MPa}$. Strain hardening on the slip systems of the crystalline lamellae is neglected, i.e. all critical resolved shear stresses are assumed to be constant. The viscoplastic rate exponent and the reference shear rate of the crystalline phase are set to $n^{\mathrm{c}}=9$ and $\dot{\gamma}_{0}^{\mathrm{c}}=0.001 \mathrm{~s}^{-1}$, after Lee et al. [22].

Table 3.2 Slip systems and the critical resolved shear stresses, corresponding to the viscoplastic power law relation [9, 22, 23].

\begin{tabular}{lcc}
\hline & Slip system & $g^{\alpha} / g_{0}$ \\
\hline Chain slip & $(100)[001]$ & 1.0 \\
& $(010)[001]$ & 2.5 \\
& $(110)[001]$ & 2.5 \\
& $(1 \overline{1} 0)[001]$ & 2.5 \\
\hline Transverse slip & $(100)[010]$ & 1.66 \\
& $(010)[100]$ & 2.5 \\
& $(110)[1 \overline{1} 0]$ & 2.2 \\
$(\overline{1} 10)[110]$ & 2.2 \\
\hline
\end{tabular}

\subsubsection{Amorphous phase}

An isotropic generalized neo-Hookean relationship is used to model the elastic behavior of the amorphous phase. The bulk modulus $K^{\mathrm{a}}$ is chosen to be 3000 $\mathrm{MPa}$ [43]. The effect of the shear and bulk moduli of the interlamellar layer on the predicted Young's modulus of the elastic composite inclusion model was discussed in [29]. Figure 3.3 presents the influence of different amorphous shear moduli on the initial behavior, predicted by the elasto-viscoplastic model, compared to experimental data [29]. Based on this observation, the shear modulus of the amorphous phase is selected to be $35 \mathrm{MPa}$. 


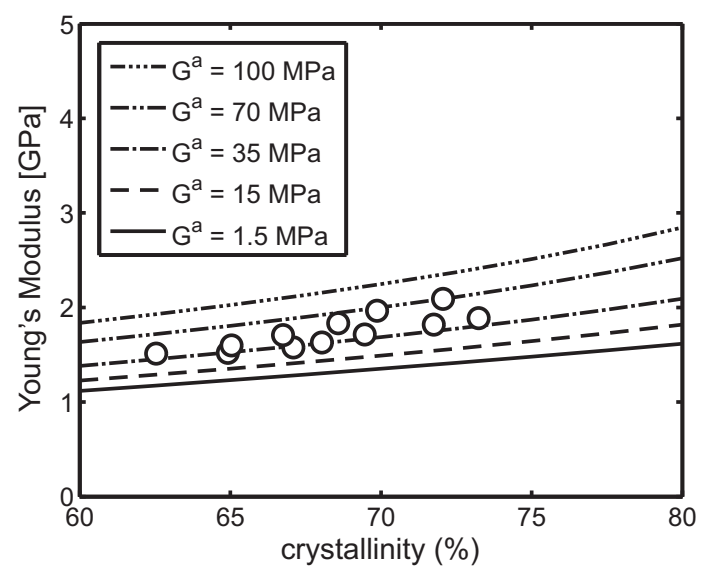

Figure 3.3 The predicted initial modulus in uniaxial compression as a function of crystallinity for different shear moduli of the amorphous phase. Symbols give experimental results of HDPE [29].

The viscoplastic rate exponent and the reference shear rate of the amorphous phase are set equal to those of the crystalline phase. The reference initial shear strength of the amorphous phase is considered to be related to the critical resolved shear stress of the easiest slip system as $g_{0}^{\mathrm{a}}=a g_{0}$. The viscoplastic parameters of the amorphous phase, used in the power law relation, and the hardening parameters, are listed in table 3.3, in which $\mu_{\mathrm{R}}$ is proportional to the initial stiffness and $N$ represents the number of rigid links between entanglements in the Arruda-Boyce eight-chain network rubber elasticity model [39].

Table 3.3 Viscoplastic and hardening parameters of the amorphous phase.

\begin{tabular}{ccccc}
\hline$\dot{\gamma}_{0}\left[\mathrm{~s}^{-1}\right]$ & $n$ & $a$ & $\mu_{\mathrm{R}}[\mathrm{MPa}]$ & $N$ \\
\hline 0.001 & 9 & 1.2 & 1.6 & 49 \\
\hline
\end{tabular}

\subsubsection{Initial crystalline and lamellar orientations}

Experimental studies of melt-crystalized PE and molecular models show that lamellar surfaces are of the $\{h 0 l\}$ type, where the angle between the chain direction $\vec{c}$ and the lamellar normal direction $\vec{n}^{I}$ varies between $20^{\circ}$ and $40^{\circ}$, 44 , 45]. Here, the lamellar surface is set to $\{201\}$, which corresponds with an angle of $35^{\circ}$. The spherulitic structure of melt-crystallized PE is represented by an aggregate of 500 randomly oriented inclusions. 


\subsubsection{Application: uniaxial tension}

In this section the mechanical response of initially isotropic HDPE subjected to uniaxial tension with a constant strain rate of $\dot{\epsilon}=0.001 \mathrm{~s}^{-1}$, is investigated. A volume crystallinity of $f^{\mathcal{C}}=64.9 \%$ is used in the model prediction. Figure 3.4 shows the predicted macroscopic true stress $\bar{\sigma}_{11}$, as a function of imposed macroscopic logarithmic strain $\dot{\epsilon} t=\ln (\lambda)$.

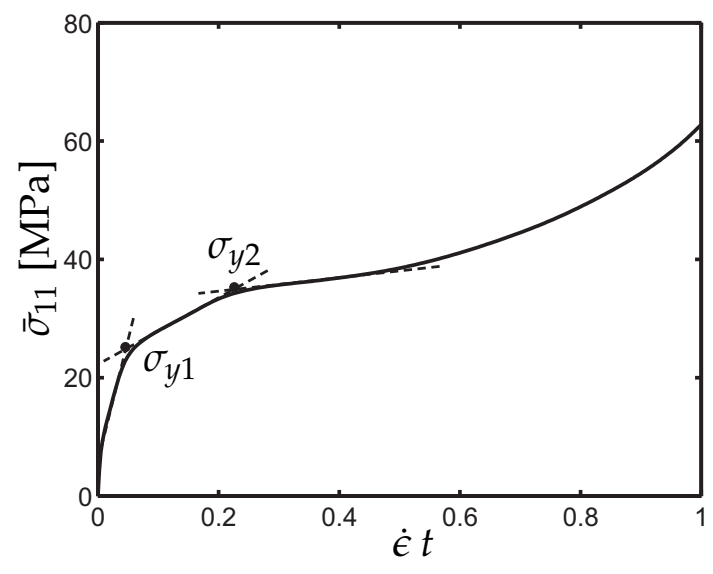

Figure 3.4 Predicted equivalent macroscopic stress vs. macroscopic logarithmic strain, for HDPE subjected to uniaxial tension. $\sigma_{y 1}$ and $\sigma_{y 2}$ indicate the first and the second yield points, respectively.

One important feature in the stress-strain response of HDPE is the existence of two yield points. The double yield phenomenon has been widely seen in engineering stress-strain curves during both tensile and compression deformation modes and reported in literature [15, 46-49]. Popli and Mandelkern [15] reported a well-resolved double tensile yield point for branched polyethylenes and ethylene copolymers at the ambient temperature. They assigned this phenomenon to the broad distribution of lamellar thickness. However, this hypothesis was refuted by Séguéla and Darras [49], who phenomenologically studied the double yield of polyethylene and related copolymers. They found that the crystallite thickness distribution is not the main factor for the double yield phenomenon, since deeply interconnected crystals of various thickness are not allowed to yield independently, giving rise to distinct yield points. A partial melting-recrystallization process was also proposed as an alternative to explain the multiple yield in polyethylene [15, 50]. It was proposed that the concentration of stress on the less perfect crystallites provides enough energy for them to partially melt and recrystallize during deformation to form a new population of crystallites. The occurrence of two yield points was then assigned 
to the yielding of the original and the newly formed crystallites [50]. However, partial melting-recrystallization is not a commonly accepted explanation for the existence of double yields. Evidence against this hypothesis was given by Butler et al. [51]. Time resolved simultaneous small- and wide-angle Xray scattering experiments [51-53] during deformation of polyethylene in both tensile and compression modes, revealed that the double yield point exists in both deformation modes. The most accepted explanation for the deformation mechanisms of both yield points is the association of the first yield point to fine slip within the crystalline lamellae and the presence of a process of coarse slip resulting in lamellar fragmentation at the second yield point [51-54], as schematically shown in figure 3.5 .

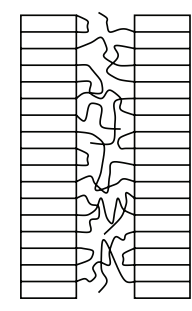

fine slip

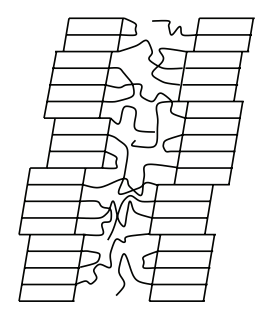

coarse slip

$\sigma_{y 2}$

Figure 3.5 Schematic representation of fine slip at the first yield point $\left(\sigma_{y 1}\right)$ accompanied by lamellar disintegration due to coarse slip at the second yield point $\left(\sigma_{y 2}\right)$ [55].

As can be seen in figure 3.4, a double yield phenomenon is also found in the model prediction, although there is no coarse slip or lamellar fragmentation present in the model since all slips are assumed to be of the fine slip type. The phenomenon observed in the model prediction must, therefore, originate from the morphological features and deformation mechanisms present in the model. Figure 3.6 shows the activity of the (100)[001] chain slip system and the transverse (100)[010] and $\{110\}\langle 110\rangle$ slip systems, represented by their normalized averaged resolved shear rate $\overline{\dot{\gamma}} / \dot{\gamma}_{0}$, during deformation. The first and the second yield point are also marked in the figure. As can be seen, since the crystallographic (100)[001] slip system is the most easily activated slip system, it is predominantly active. Up to the first yield point, crystallographic slip is mainly limited to the (100)[001] chain slip system, while the transverse slip systems reveal very weak activity up to this point. After the first yield point, the activity of the transverse slip systems increases up to the second yield point and significant crystallographic slip takes place in the transverse slip systems, 
particularly the $\{110\}\langle 1 \overline{1} 0\rangle$ family of transverse slip systems and these modes of deformation become highly active. It is worth pointing out that the $\{110\}\langle 1 \overline{1} 0\rangle$ family of slip systems become active not in all inclusions, but in particular ones, oriented at an optimum angle for these slip systems to become active. This can be concluded from the relatively large value of the standard deviation of the normalized resolved shear rate of the $\{110\}\langle 1 \overline{1} 0\rangle$ family of transverse slip systems at the second yield point. This change of mechanism between the chain and transverse slip systems is found to be responsible for the double yield phenomenon present in the model and, therefore, the difference between the level of the critical resolved shear stress of the (100)[001] slip system and the $\{110\}\langle 1 \overline{1} 0\rangle$ family of slip systems determines the difference between the two yield points and the strain hardening modulus between them.

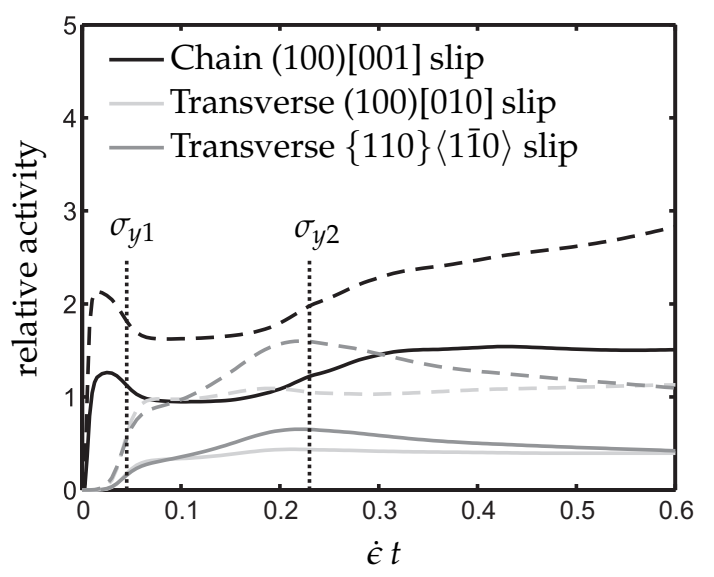

Figure 3.6 Activity of the three easiest crystallographic deformation modes vs. macroscopic logarithmic strain in uniaxial tension. Solid lines show the normalized averaged resolved shear rate $\bar{\gamma} / \dot{\gamma}_{0}$ and dashed lines show the normalized values plus their corresponding standard deviations. $\sigma_{y 1}$ and $\sigma_{y 2}$ represent the first and the second yield points, respectively.

\subsection{Characterization of model parameters}

Since localization phenomena like necking and crazing, which occur in uniaxial tensile experiments, are not present in uniaxial compression tests, these tests are usually employed to determine the intrinsic deformation behavior of polymers [55, 56]. In accordance with that, uniaxial compression experiments, performed at various strain rates on HDPE (Stamylan HD 9089S) at room temperature, are used here to characterize the elasto-viscoplastic model parameters. 
The kinetics of plastic flow of semicrystalline polymers at a macroscopic level are mainly governed by the kinetics of the crystallographic slip systems together with the yield kinetics of the amorphous domains at the microscopic scale. Therefore, a suitable description of the rate-dependency of slip along crystallographic planes as well as the rate-dependency of the effective shear stress of the amorphous phase, enables a quantitative prediction of the mechanical performance of semicrystalline polymers. The strain rate dependency of the compressive yield stress, predicted by a viscoplastic power law relation, is shown in figure 3.7b. It is observed that the model does not provide a good prediction of experimental data.

As a first step in achieving a quantitative prediction, the previously used power law relation is replaced with a viscoplastic Eyring flow rule. The initial values of the reference shear rate $\dot{\xi}_{0}^{\alpha}$ and the reference shear strength $\tau_{0}^{\mathrm{c}}$ in the Eyring flow rule are determined by a nonlinear least-squares curve fitting of the power law relation in the range of $10^{-3}-10^{-1}$ (see figure $3.7 \mathrm{a}$ ). The strain rate dependency of the yield stress, predicted by the elasto-viscoplastic model with the Eyring flow rule, is presented in figure $3.7 \mathrm{~b}$.

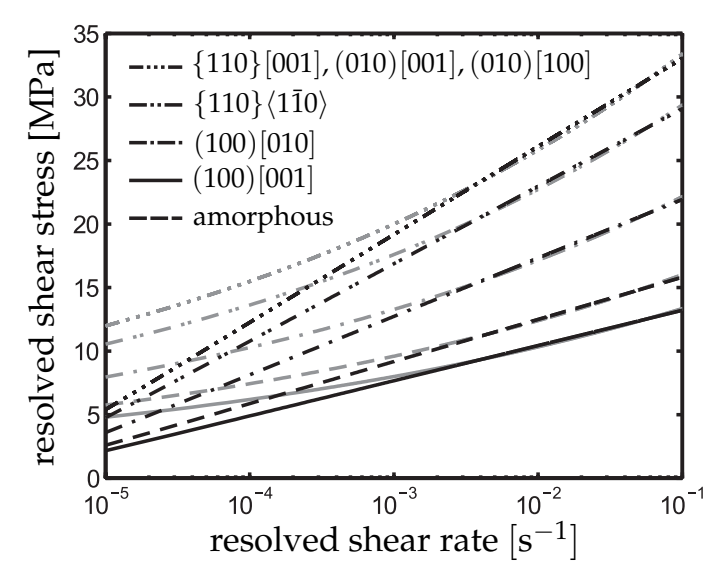

a

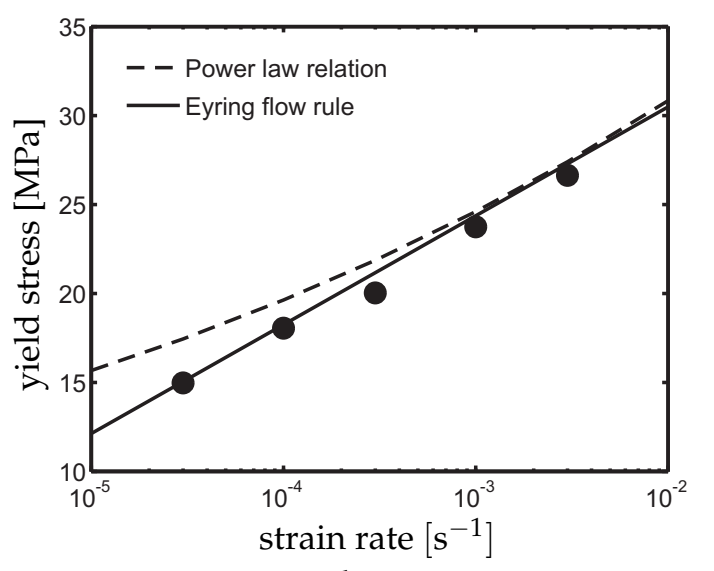

b

Figure 3.7 (a) Description of slip kinetics of individual slip systems using the power law relation (gray lines) and the Eyring flow rule (black lines). (b) Strain rate-dependency of the compressive yield stress, predicted by the elasto-viscoplastic model with both a power law relation and an Eyring flow rule. Symbols show the compressive yield stress of quenched HDPE with a volume crystallinity of $f^{c}=64.9 \%$ at room temperature, which was also used in the model prediction.

However, true stress-strain curves obtained from compression tests on HDPE samples with a varying degree of crystallinity and strain rate show that both yield stresses increase in a similar way, resulting in a constant strain hardening modulus between the two yield points, as shown in figure 3.8. Since the harden- 
ing between the yield points predicted by the model is mainly governed by the difference between the kinetics of the (100)[001] slip system and the $\{110\}\langle 1 \overline{1} 0\rangle$ family of slip systems, the slip kinetics of the individual slip systems should be parallel on a semi-logarithmic scale. The slip kinetics are, therefore, refined and

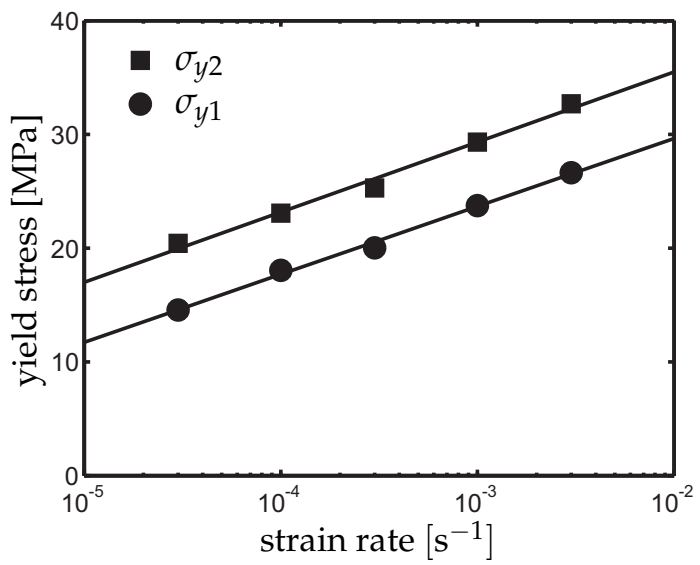

Figure 3.8 First $\left(\sigma_{y 1}\right)$ and second $\left(\sigma_{y 2}\right)$ yield stresses as a function of strain rate for HDPE samples at room temperature. Lines are a guide to the eye to indicate the strain ratedependence.

re-evaluated in terms of the reference shear rate $\dot{\xi}_{0}^{\alpha}$ and the characteristic shear stress $\tau_{0}^{\mathrm{c}}$ in order to fit the compressive true stress-strain curves of HDPE. To do that, the value of the characteristic shear stress of the amorphous and the crystalline phase in the Eyring model, are set to an equal value of $\tau_{0}^{\mathrm{c}}=\tau_{0}^{\mathrm{a}}=1.2$ MPa. Moreover, the reference shear rate of the amorphous phase is taken to be $\dot{\xi}_{0}^{a}=6.6 \times 10^{-7}\left[\mathrm{~s}^{-1}\right]$. Table 3.4 summarizes the reference shear rate $\dot{\xi}_{0}^{\alpha}$ for individual slip systems.

Compressive true stress-strain curves at varying strain rates, predicted by the model using the refined slip kinetics are shown in figure 3.9. Comparison with experimental data reveals a good agreement up to moderate strains. It should be noted that the post second-yield behavior is not predicted as accurately as the post first-yield response. A very likely cause is the fact that lamellar fragmentation, due to the process of coarse slip, is not taken into account in the model. The model prediction of the relation between crystallinity and the compressive first and second yield stress is also presented in figure 3.10. Both yield points are increasing in a similar way as also observed in experimental data.

The behavior of the amorphous phase also plays an important role in the yield kinetics. At room temperature, the amorphous phase of HDPE should be in the 
Table 3.4 Slip systems and the reference shear rates, corresponding to the viscoplastic Eyring flow rule.

\begin{tabular}{lcc}
\hline & Slip system & $\dot{\xi}_{0}^{\alpha}\left[\mathrm{s}^{-1}\right]$ \\
\hline Chain slip & $(100)[001]$ & $2.5 \times 10^{-6}$ \\
& $(010)[001]$ & $1.3 \times 10^{-10}$ \\
& $(110)[001]$ & $1.3 \times 10^{-10}$ \\
& $(1 \overline{1} 0)[001]$ & $1.3 \times 10^{-10}$ \\
\hline Transverse slip & $(100)[010]$ & $1.0 \times 10^{-7}$ \\
& $(010)[100]$ & $1.3 \times 10^{-10}$ \\
& $(110)[1 \overline{1} 0]$ & $2.0 \times 10^{-9}$ \\
$(\overline{1} 10)[110]$ & $2.0 \times 10^{-9}$ \\
\hline
\end{tabular}

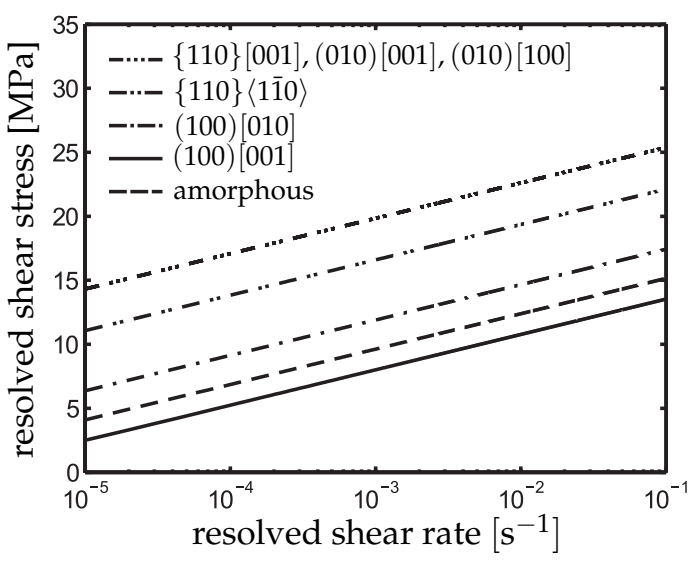

a

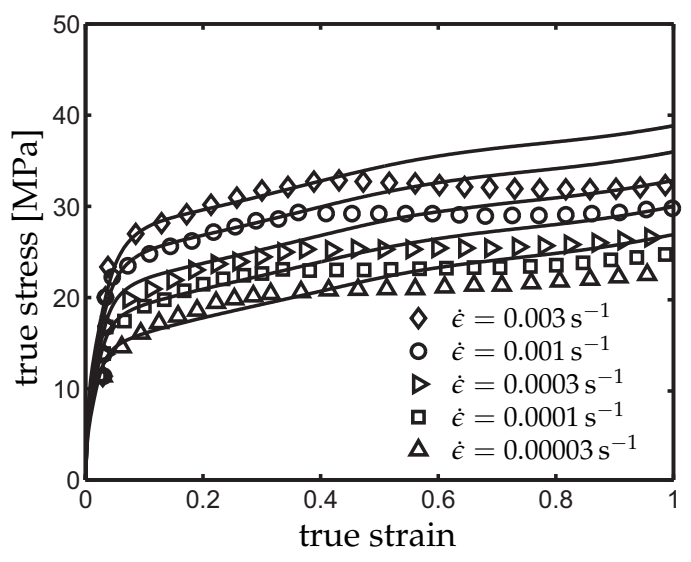

$\mathrm{b}$

Figure 3.9 (a) Description of slip kinetics of individual slip systems by using the Eyring flow rule. (b) The predicted compressive true stress-strain curves (solid lines) compared to experimental true stress-strain curves (symbols) for quenched HDPE with $X_{v o l}=64.9 \%$ at room temperature.

rubbery regime, with the glass transition temperature near $-70{ }^{\circ} \mathrm{C}$. However, as already mentioned, an elasto-viscoplastic behavior was assumed for the amorphous phase. In order to test the validity of this assumption, the mechanical response of HDPE subjected to uniaxial compression was simulated with the amorphous phase considered to behave elastically with the shear modulus equal to that of the bulk amorphous material [29]. The levels of the resolved shear stress 


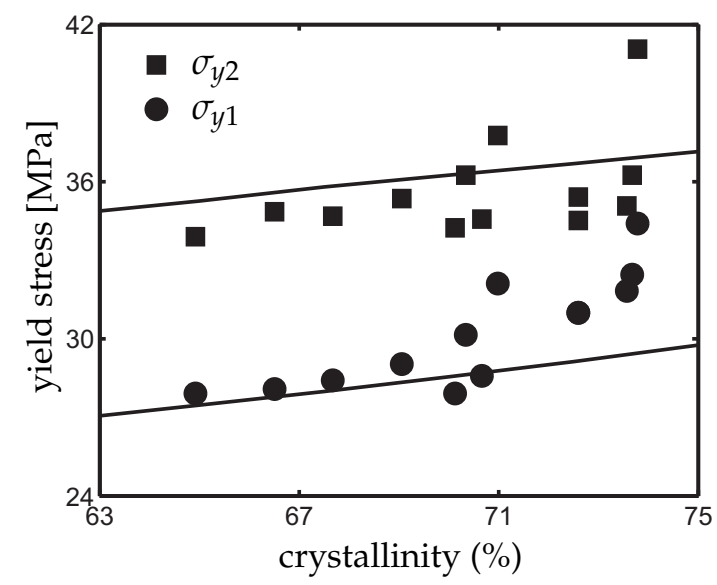

Figure 3.10 Relationship between the degree of crystallinity and the two yield points predicted by the model (solid lines), compared to experimental data [55] (symbols), for HDPE at a constant strain rate $\dot{\epsilon}=0.003 \mathrm{~s}^{-1} . \sigma_{y 1}$ and $\sigma_{y 2}$ indicate the first and the second yield points, respectively.

of all individual slip systems were then increased in order to fit the compressive experimental data with this new assumption. The results showed that the first yield point, which is mainly due to the activity of the (100)[001] chain slip system, was still predicted well, however it resulted in an unrealistically high second yield point.

As has already been noted, the difference between the level of the first and second yield points is governed by the level of the resolved shear stresses of the (100)[001] chain slip and the $\{110\}\langle 1 \overline{1} 0\rangle$ family of transverse slip systems. Therefore, an attempt was made to enhance the prediction of the second yield point in case of a fully elastic amorphous phase by decreasing the slip kinetics of the $\{110\}\langle 1 \overline{1} 0\rangle$ family of transverse slip systems. However, this situation could only occur when it is unrealistically assumed that the $\{110\}\langle 1 \overline{1} 0\rangle$ transverse slip systems are the easiest slip systems rather than the (100)[001] chain slip system. In this case, the first yield point is still governed mainly by (100)[001] chain slip and the $\{110\}\langle 1 \overline{1} 0\rangle$ family of transverse slip systems, which have become the easiest slip systems, are not activated. This is due to the initially fixed relation between the crystalline orientation and the lamellar surface, which is taken to be of the $\{201\}$ type. The above consideration, as well as the high value of the relaxed modulus associated with the glass-rubber relaxation [36], and the presence of a chain diffusion process [37], supports the choice of an elasto-viscoplastic behavior for the interlamellar material.

The texture evolution during deformation is depicted in figure 3.11. As can be seen, the normals to the crystallographic (100) plane, or $\vec{a}$ axes, migrate towards 
the compression direction, whereas the normals to the crystallographic (010) planes, or $\vec{b}$ axes, orient to an angle of almost $70^{\circ}-80^{\circ}$ with the compression direction, and the normals to the (001) planes, or $\vec{c}$ axes, migrate away from the compression axis. The lamellar normals are also observed to migrate towards the compression axis. Evolution of crystallographic and morphological textures

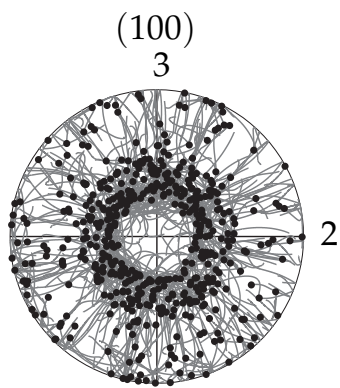

a

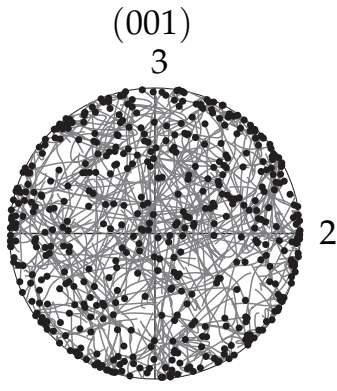

C

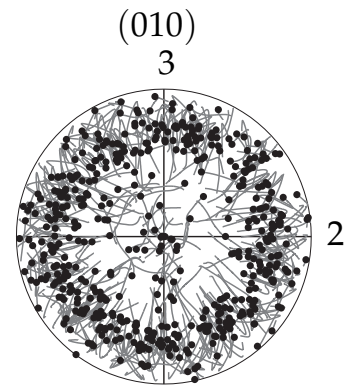

b

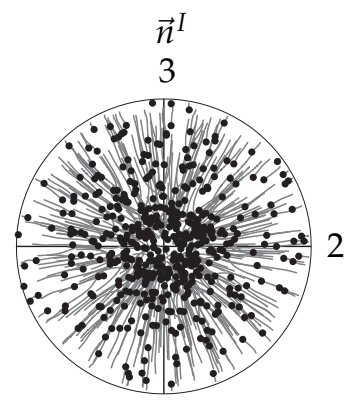

d

Figure 3.11 Predicted crystallographic and morphological textures trajectories for HDPE subjected to uniaxial compression up to a macroscopic logarithmic strain of $\bar{\epsilon}=1$. The dots denote the final position of the poles.

has a great influence on the mechanical behavior of a semicrystalline polymer. Therefore, a prediction of the evolution of macroscopic mechanical properties depends on the prediction of the evolution of textural anisotropy. Here, the texture development predicted by the model, is confronted with some limited experimental data. Figure 3.12 shows the predicted textures, compared to experimental WAXS intensities reported by Bartczak et al. [8]. It is observed that the normals of the (100) planes migrate toward the compression direction, whereas the normals of the (010) planes rotate away from this axis. Experimental WAXS intensities show a maximum for (100) planes located at an angle of $25^{\circ}-30^{\circ}$ with the compression direction, which is in good agreement with the predicted 


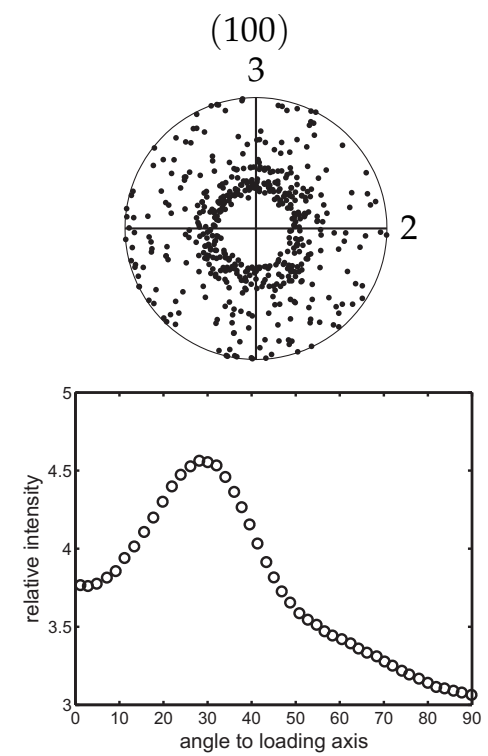

a

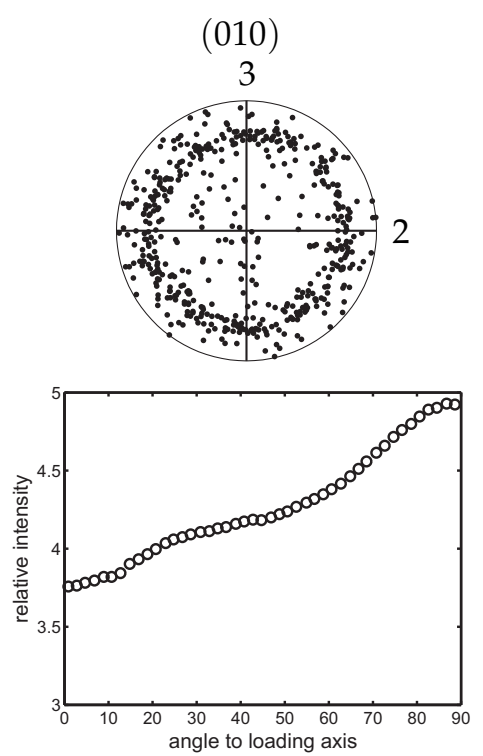

b

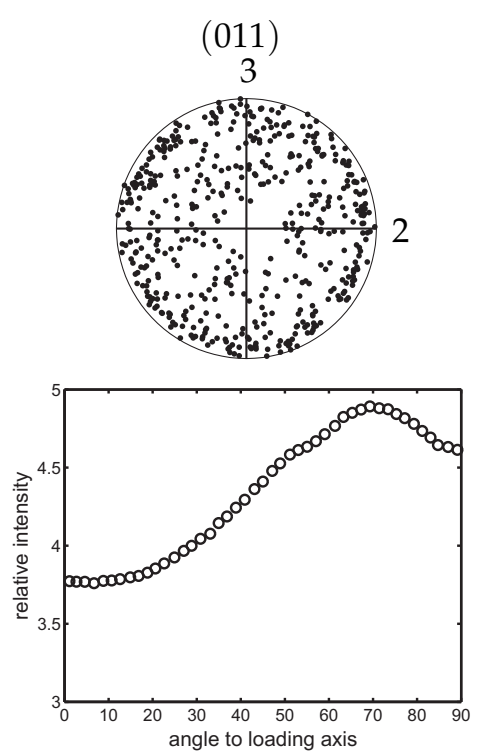

C

Figure 3.12 Top: predicted crystallographic textures for HDPE subjected to uniaxial compression at a macroscopic true strain of $\bar{\epsilon}=0.82$. The compression direction is normal to the plane of the pole figures. Bottom: corresponding experimental WAXS intensities after [8].

orientation of the (100) planes. For the (010) planes, the experimental maximum intensity is located at $80^{\circ}-90^{\circ}$. The model prediction also shows these poles to migrate away from the compression direction, however the maximum intensity of poles is located at an angle of almost $70^{\circ}-80^{\circ}$. The (011) planes show a weak texture, however the general tendency for these planes is to rotate away from the compression direction. The morphological texture predictions and their corresponding SAXS patterns are shown in figure 3.13. As can be seen, the tendency of lamellar normals to migrate towards the compression direction during deformation is compared favorably with the experimental data, obtained by Bartczak et al. [8].

As already mentioned, the double yield phenomenon, found in the model, is related to the morphological factors that induce a change of deformation mechanisms. Here, the texture prediction is used to analyze the underlying morphological changes. Figure 3.14 a represents the true stress-strain behavior of HDPE during uniaxial compression with a constant strain rate of $\dot{\epsilon}=0.001 \mathrm{~s}^{-1}$. Crystallographic texture ( $\vec{b}$ axis, corresponding to the lamellar growth direction) and morphological texture at four different points are studied in figure $3.14 \mathrm{~b}$. 


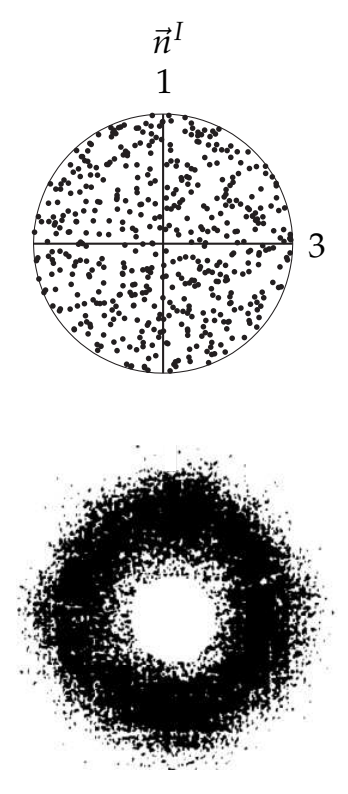

a

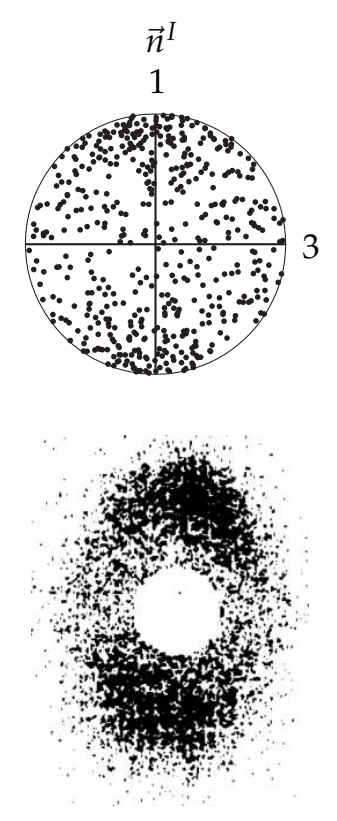

b

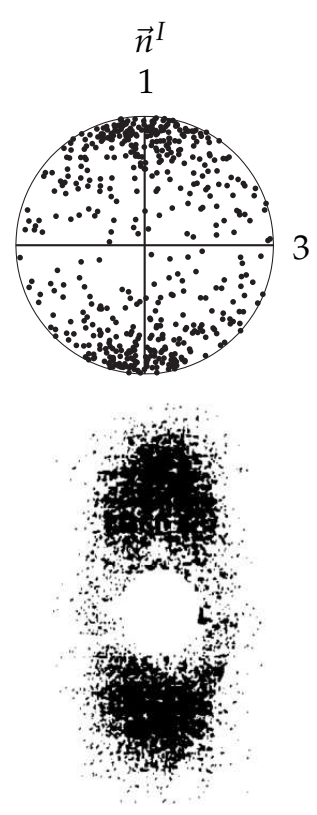

C

Figure 3.13 Top: predicted morphological textures for HDPE subjected to uniaxial compression at a macroscopic true strain of (a) $\bar{\epsilon}=0$, (b) $\bar{\epsilon}=0.35$ and (c) $\bar{\epsilon}=0.82$. The compression direction is vertical. Bottom: Corresponding experimental SAXS intensities [8].

In these pole figures, the location of each dot denotes the orientation of an inclusion and its gray value shows the magnitude of the resolved shear rate of the indicated slip system for the inclusion. As can be seen, up to the first yield point (point 2), crystallographic slip occurs predominantly on the (100)[001] slip system, which is the most easily activated slip system, and specifically for those inclusions whose lamellar normals are almost aligned with the compression direction, and with lamellar growth directions perpendicular to that. The microscopic deformation, therefore, is dependent on the local orientation of the lamellar normals with respect to the loading direction. Lamellae with their normals aligned in the compression direction are at an optimum orientation for the (100)[001] slip system to become active. The $\{110\}\langle 1 \overline{1} 0\rangle$ family of slip systems shows little activity up to this point. At the second yield point (point 4), significant crystallographic slip takes place in the transverse slip systems, particularly the $\{110\}\langle 1 \overline{1} 0\rangle$ family of transverse slip systems, for inclusions with the growth direction almost perpendicular to the compression direction and the lamellar normal direction aligned to the loading axis. The change of mechanism between the chain and transverse slip systems is found to be responsible for 
the double yield phenomenon present in the model. This morphological change might also be the underlying mechanism for the onset of lamellar break up.

\subsection{Discussions and conclusions}

The deformation of semicrystalline polymers is the result of the operation of various mechanisms at different levels. An accurate quantitative prediction of the mechanical behavior of these materials requires a good description and modeling of the various deformation mechanisms in the heterogeneous microstructure. The layered two-phase, micromechanical model is able to simulate the elastoviscoplastic properties of semicrystalline polymers based on the micromechanics of the material. At the microscopic level, a two-phase composite entity is employed, which is comprised of a crystalline lamella that plastically deforms by rate-dependent slip, and an amorphous phase, with a rate-dependent flow process. At the macroscopic level, the material is modeled by an aggregate of a number of composite inclusions.

An attempt was made to add quantitative predictive abilities to the model. To do that, slip kinetics of the individual crystallographic slip systems, being responsible for time-dependent macroscopic failure, have been re-evaluated and refined by using a numerical/experimental approach. It has also been illustrated that an Eyring flow rule can better mimic the kinetics of the macroscopic plastic flow, and, therefore, the previously used viscoplastic power law relation has been replaced with an Eyring flow rule. The necessity of using a viscoplastic behavior for the amorphous phase has been discussed as well. Comparing the predicted stress-strain behavior of HDPE with experimental data, shows a promising agreement.

A double yield phenomenon has also been observed in the model prediction and has been found to originate from the morphological changes during deformation that cause a change in the deformation mechanisms. Predicted texture evolution during deformation, has been used to analyze and understand the morphological factors, resulting in a double yield phenomenon in the model. Confrontation of the predicted two yield points with experimental data shows that the model provides a good prediction of the relation between crystallinity and the first yield point. However, the second yield point and the second post-yield behavior have not been predicted as accurately as the first, since the lamellar disintegration, which is a consequence of the process of coarse slip, has not been taken into account in the model.

Figure 3.15 illustrates the predicted true tensile stress-strain curves showing double yield points together with their corresponding engineering stress-strain 


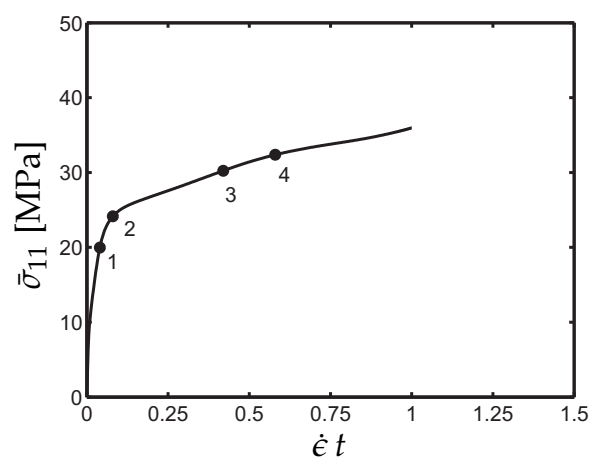

a

(1)
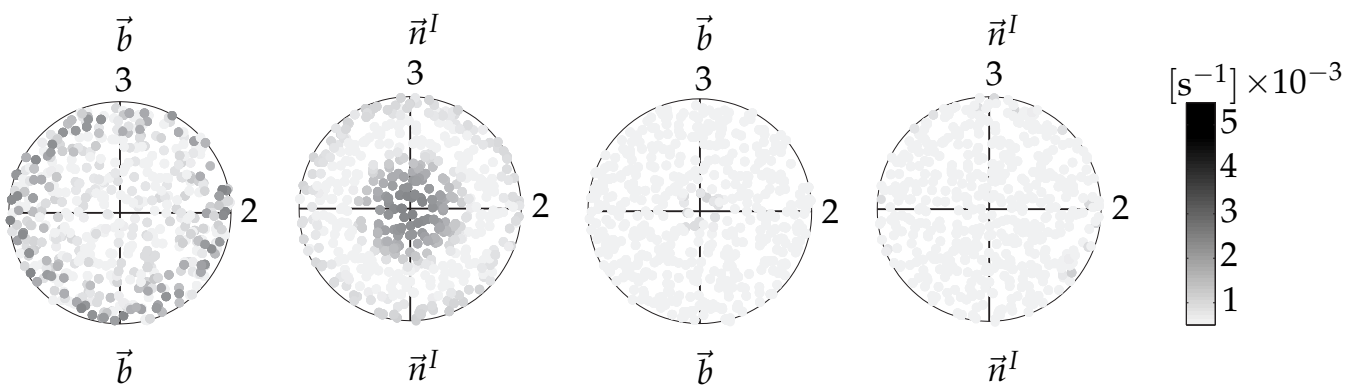

(2)
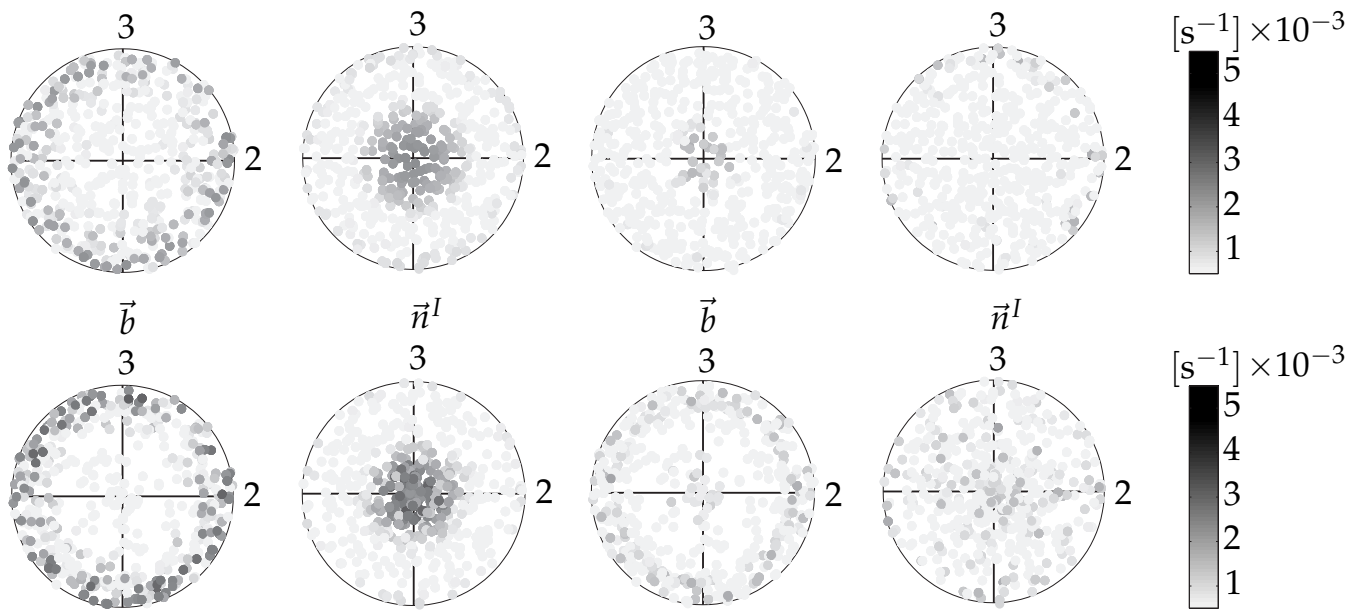

$\vec{b}$

$\vec{n}^{I}$
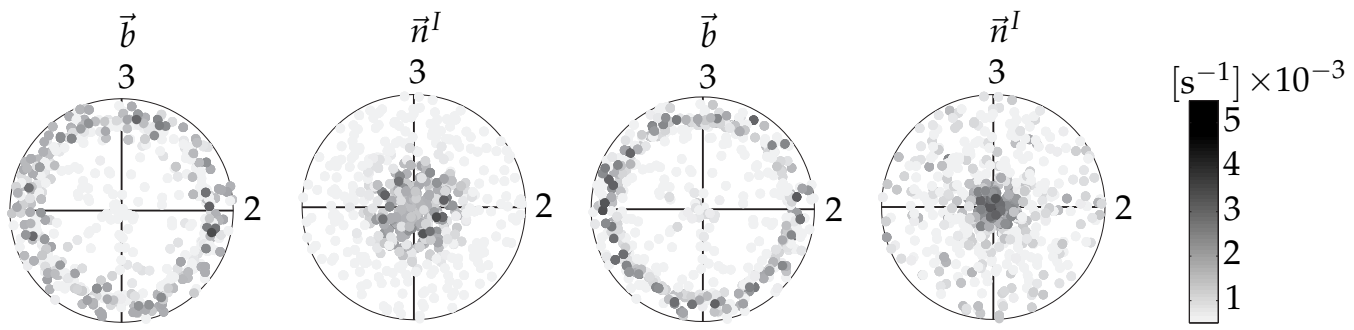

I

II

III

IV

$\mathrm{b}$

Figure 3.14 (a) True stress-true strain curve for HDPE, subjected to uniaxial compression. (b) Activity of (I)-(II) the (100)[001] slip system, and (III)-(IV) the $\{110\}\langle 1 \overline{1} 0\rangle$ slip systems, at different strain. Gray intensity represents the magnitude of the resolved shear rate of the specified slip system for each inclusion. 
curves. One of the consequences of double yield points is that the second yield

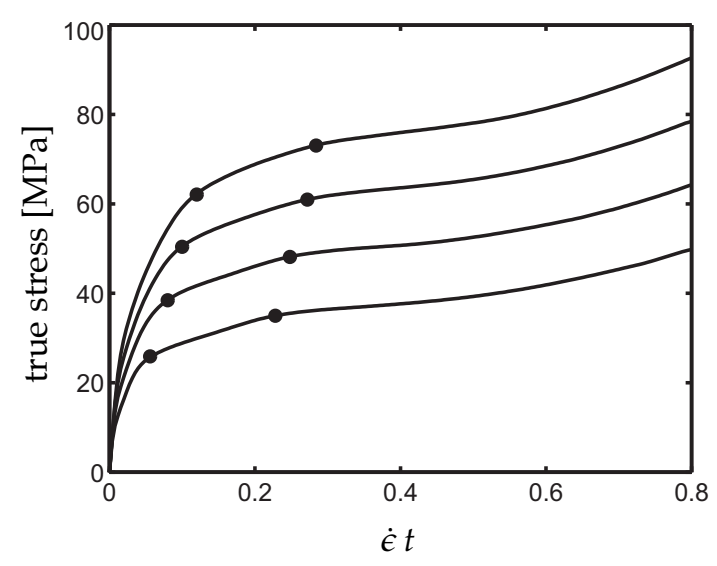

a

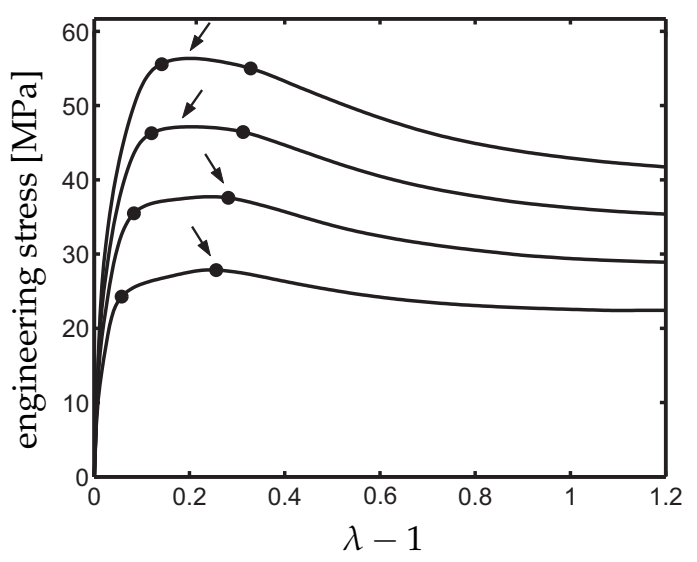

$\mathrm{b}$

Figure 3.15 Model prediction of (a) true stress-strain curves and (b) engineering stressstrain curves, exhibiting double yield points in uniaxial tension.

point always leads to a sharp neck for tensile loading, whereas the first yield point is associated either with no neck or with only a very shallow neck due to the relatively large strain hardening following the first yield point [46]. As a result, the experimental tensile yield stress, defined as a local maximum in stress can either be related to the first yield or the second yield stress as depicted with the arrows in figure $3.15 \mathrm{~b}$, and studies using the yield point from tensile tests can become less unambiguous. Experimental tensile tests on polyethylene and related copolymers [46, 49], performed at different temperatures, revealed the same shape of yielding as the true stress-strain curves of figure $3.15 \mathrm{~b}$. Besides temperature, strain rate and crystallinity also have an effect on the yield stress. It is noteworthy that a more accurate prediction requires a re-evaluation of the slip kinetics, including their dependence on pressure and lamellar thickness as well. 


\section{A Appendix: Micromechanical model}

In this appendix, different aspects of the composite inclusion model for semicrystalline polymers are presented.

\section{A.1 Crystalline phase}

The elastic behavior of the crystalline phase is characterized by a fourth order elasticity tensor ${ }^{4} \mathcal{C}^{\mathrm{C}}$ which linearly relates the second Piola-Kirchhoff stress tensor $\tau^{\mathrm{C}}$ and the Green-Lagrange strain tensor $\boldsymbol{E}_{\mathrm{e}}^{\mathrm{c}}$ :

$$
\tau^{\mathrm{c}}={ }^{4} \mathcal{C}^{\mathrm{c}}: E_{\mathrm{e}^{\prime}}^{\mathrm{c}}
$$

with

$$
\boldsymbol{\tau}^{\mathrm{c}}=J_{\mathrm{e}}^{\mathrm{c}} \boldsymbol{F}_{\mathrm{e}}^{\mathrm{c}^{-1}} \cdot \sigma^{\mathrm{c}} \cdot \boldsymbol{F}_{\mathrm{e}}^{\mathrm{c}^{-\mathrm{T}}} \quad \text { and } \quad \boldsymbol{E}_{\mathrm{e}}^{\mathrm{c}}=\frac{1}{2}\left(\boldsymbol{F}_{\mathrm{e}}^{\mathrm{c}^{\mathrm{T}}} \cdot \boldsymbol{F}_{\mathrm{e}}^{\mathrm{c}}-\boldsymbol{I}\right),
$$

with $J_{\mathrm{e}}^{\mathrm{c}}=\operatorname{det}\left(\boldsymbol{F}_{\mathrm{e}}^{\mathrm{c}}\right)$ the volume ratio, $\sigma^{\mathrm{c}}$ the Cauchy stress tensor and $\boldsymbol{I}$ the second order identity tensor. The viscoplastic component of the deformation in the crystalline phase is given by:

$$
\boldsymbol{L}_{\mathrm{p}}^{\mathrm{c}}=\dot{\boldsymbol{F}}_{\mathrm{p}}^{\mathrm{c}} \cdot \boldsymbol{F}_{\mathrm{p}}^{\mathrm{c}-1}=\sum_{\alpha=1}^{N_{s}} \dot{\gamma}^{\alpha} \boldsymbol{P}_{0}^{\alpha} \quad ; \quad \boldsymbol{P}_{0}^{\alpha}=\vec{s}_{0}^{\alpha} \otimes \vec{n}_{0}^{\alpha},
$$

with

$$
\dot{\gamma}^{\alpha}=\dot{\xi}_{0}^{\alpha} \sinh \left[\frac{\tau^{\alpha}}{\tau_{0}^{\mathrm{c}}}\right] \text { and } \tau^{\alpha}=\tau^{\mathrm{c}} \cdot \mathrm{C}_{\mathrm{e}}^{\mathrm{c}}: \boldsymbol{P}_{0}^{\alpha} .
$$

where $\mathrm{C}_{\mathrm{e}}^{\mathrm{c}}$ denotes the elastic right Cauchy-Green deformation tensor.

\section{A.2 Amorphous phase}

The elastic behavior of the amorphous phase is modeled by a generalized neoHookean relationship:

$$
\sigma_{\mathrm{e}}^{\mathrm{a}}=\frac{G^{\mathrm{a}}}{J_{\mathrm{e}}^{\mathrm{a}}} \tilde{\boldsymbol{B}}_{\mathrm{e}}^{\mathrm{a}^{\mathrm{d}}}+K^{\mathrm{a}}\left(J_{\mathrm{e}}^{\mathrm{a}}-1\right) \boldsymbol{I},
$$


where the superscript "d" denotes the deviatoric part, $\tilde{\boldsymbol{F}}_{\mathrm{e}}^{\mathrm{a}}=\int_{\mathrm{e}}^{\mathrm{a}-\frac{1}{3}} \boldsymbol{F}_{\mathrm{e}}^{\mathrm{a}}$ is the isochoric elastic deformation gradient tensor and $\tilde{\boldsymbol{B}}_{\mathrm{e}}^{\mathrm{a}}=\tilde{\boldsymbol{F}}_{\mathrm{e}}^{\mathrm{a}} \cdot \tilde{\boldsymbol{F}}_{\mathrm{e}}^{\mathrm{a}^{\mathrm{T}}}$ is the isochoric elastic left Cauchy-Green deformation tensor. $G^{\mathrm{a}}$ and $J_{\mathrm{e}}^{\mathrm{a}}$ are the shear modulus and bulk modulus, respectively.

A viscoplastic Eyring flow rule is employed to relate the effective shear strain rate $\dot{\gamma}_{\mathrm{p}}^{\mathrm{a}}$ to the effective shear stress of the amorphous phase $\tau^{\mathrm{a}}$, defined as

$$
\tau^{\mathrm{a}}=\sqrt{\frac{1}{2} \sigma_{*}^{\mathrm{a}^{\mathrm{d}}}: \sigma_{*}^{\mathrm{a}^{\mathrm{d}}}} \quad \text { with } \quad \sigma_{*}^{\mathrm{a}}=\boldsymbol{R}_{\mathrm{e}}^{\mathrm{a}^{\mathrm{T}}} \cdot \sigma^{\mathrm{a}} \cdot \boldsymbol{R}_{\mathrm{e}}^{\mathrm{a}}-H^{\mathrm{a}},
$$

with $\boldsymbol{R}_{\mathrm{e}}^{\mathrm{a}}$ the rotation tensor, obtained from the polar decomposition of $\boldsymbol{F}_{\mathrm{e}}^{\mathrm{a}}$ and $\boldsymbol{H}^{\mathrm{a}}$ a back stress tensor, which accounts for orientation-induced hardening and is given by:

$$
\boldsymbol{H}^{\mathrm{a}}=\mu_{\mathrm{R}} \frac{\sqrt{N}}{\lambda_{\mathrm{ch}}} \mathcal{L}^{-1}\left(\frac{\lambda_{\mathrm{ch}}}{\sqrt{N}}\right)\left(\boldsymbol{B}_{\mathrm{p}}^{\mathrm{a}}-\lambda_{\mathrm{ch}}^{2} \boldsymbol{I}\right),
$$

where $\lambda_{\mathrm{ch}}=\sqrt{\frac{1}{3} \operatorname{tr}\left(B_{\mathrm{p}}^{\mathrm{a}}\right)}$ represents the stretch of each chain in the eight-chain network model and $\mathcal{L}^{-1}$ is the inverse of the Langevin function. The plastic rate of deformation $D_{\mathrm{p}}^{\mathrm{a}}$ is then given by:

$$
D_{\mathrm{p}}^{\mathrm{a}}=\frac{\dot{\gamma}_{\mathrm{p}}^{\mathrm{a}}}{\tau^{\mathrm{a}}} \sigma_{*}^{\mathrm{a}^{\mathrm{d}}}
$$

\section{A.3 Composite inclusion}

The inclusion-averaged deformation gradient $\boldsymbol{F}^{\mathrm{I}}$ and Cauchy stress $\sigma^{\mathrm{I}}$ of each individual composite are given by:

$$
\begin{aligned}
& \boldsymbol{F}^{\mathrm{I}}=f_{0}^{\mathrm{c}} \boldsymbol{F}^{\mathrm{c}}+\left(1-f_{0}^{\mathrm{c}}\right) \boldsymbol{F}^{\mathrm{a}}, \\
& \boldsymbol{\sigma}^{\mathrm{I}}=f^{\mathrm{c}} \boldsymbol{\sigma}^{\mathrm{c}}+\left(1-f^{\mathrm{c}}\right) \boldsymbol{\sigma}^{\mathrm{a}} .
\end{aligned}
$$

Let the following fourth-order subspace projection tensors, based on the orientation of the normal $\vec{n}^{\mathrm{I}}$ of the amorphous/crystalline interface plane, be defined as:

$$
{ }^{4} \mathcal{P}_{\mathrm{n}}^{\mathrm{I}}=\sum_{i=1}^{3} \vec{e}_{i}^{\mathrm{I}} \otimes \vec{n}^{\mathrm{I}} \otimes \vec{n}^{\mathrm{I}} \otimes \vec{e}_{i}^{\mathrm{I}},
$$




$$
{ }^{4} \mathcal{P}_{\mathrm{x}}^{\mathrm{I}}={ }^{4} \mathcal{I}-{ }^{4} \mathcal{P}_{\mathrm{n}}^{\mathrm{I}}
$$

where ${ }^{4} \mathcal{I}$ is the fourth-order identity tensor. Then, the interface conditions can be written as:

$$
\begin{aligned}
& { }^{4} \mathcal{P}_{\mathrm{x}_{0}}^{\mathrm{I}}: \boldsymbol{F}^{\mathrm{a}}={ }^{4} \mathcal{P}_{\mathrm{x}_{0}}^{\mathrm{I}}: \boldsymbol{F}^{\mathrm{C}}={ }^{4} \mathcal{P}_{\mathrm{x}_{0}}^{\mathrm{I}}: \boldsymbol{F}^{\mathrm{I}} . \\
& { }^{4} \mathcal{P}_{\mathrm{n}}^{\mathrm{I}}: \sigma^{\mathrm{a}}={ }^{4} \mathcal{P}_{\mathrm{n}}^{\mathrm{I}}: \sigma^{\mathcal{C}}={ }^{4} \mathcal{P}_{\mathrm{n}}^{\mathrm{I}}: \sigma^{\mathrm{I}} .
\end{aligned}
$$

\section{A.4 Hybrid interaction law}

In the hybrid interaction model, six auxiliary deformation-like unknowns $\hat{U}$ are introduced. The prescribed components of macroscopic Cauchy stress tensor $\bar{\sigma}$ and macroscopic right stretch tensor $\bar{U}$ are denoted by ${ }^{4} \mathcal{P}_{\sigma}: \bar{\sigma}$ and ${ }^{4} \mathcal{P}_{\mathrm{U}}: \overline{\boldsymbol{U}}$, respectively. Then, for an aggregate of $N^{\mathrm{I}}$ composite inclusions, the following nonlinear equations together with the interface conditions (3.A.13) and (3.A.14) are simultaneously solved for each time increment:

- Inter-inclusion equilibrium:

$$
{ }^{4} \mathcal{P}_{\mathrm{X}}^{\mathrm{I}^{i}}: \sigma^{\mathrm{I}^{i}}={ }^{4} \mathcal{P}_{\mathrm{X}}^{\mathrm{I}^{i}}: \bar{\sigma} \quad ; \quad i=1, \ldots, N^{\mathrm{I}}
$$

- Volume-averaging of stress:

$$
\overline{\boldsymbol{\sigma}}=\sum_{i=1}^{N^{\mathrm{I}}} f^{\mathrm{I}^{i}} \sigma^{\mathrm{I}^{i}} .
$$

- Inter-inclusion compatibility:

$$
{ }^{4} \mathcal{P}_{\mathrm{n}_{0}}^{\mathrm{I}^{i}}: \boldsymbol{U}^{\mathrm{I}^{i}}={ }^{4} \mathcal{P}_{\mathrm{n}_{0}}^{\mathrm{I}^{i}}: \hat{\boldsymbol{U}} \quad ; \quad i=1, \ldots, N^{\mathrm{I}} .
$$

- Volume-averaging of deformation:

$$
\begin{aligned}
& { }^{4} \mathcal{P}_{\mathrm{U}}: \overline{\boldsymbol{U}}={ }^{4} \mathcal{P}_{\mathrm{U}}:\left(\frac{\bar{J}}{J_{\Sigma}}\right){ }^{\frac{1}{3}} \sum_{i=1}^{N^{\mathrm{I}}} f_{0}^{\mathrm{I}^{i}} \boldsymbol{U}^{\mathrm{I}^{i}}, \\
& \text { with } \bar{J}=\sum_{i=1}^{N^{\mathrm{I}}} f_{0}^{\mathrm{I}^{i}} J^{\mathrm{I}^{i}} \text { and } J_{\Sigma}=\operatorname{det}\left(\sum_{i=1}^{N^{\mathrm{I}}} f_{0}^{\mathrm{I}^{i}} \boldsymbol{F}^{\mathrm{I}^{i}}\right) .
\end{aligned}
$$


- Prescribed rotations:

$$
\boldsymbol{R}^{\mathrm{I}^{i}}=\overline{\boldsymbol{R}} \quad ; \quad i=1, \ldots, N^{\mathrm{I}} .
$$




\section{References}

[1] F.C. Frank, A. Keller, A. O'Connor, and H.H. Wills. Deformation processes in polyethylene interpreted in terms of crystal plasticity. Philosophical Magazine 25:64-74, 1958.

[2] G. Meinel, N. Riorosoff, and A. Peterlin. Plastic deformation of polyethylene. I. Change of morphology during drawing of polyethylene of high density. Journal of Polymer Science, Part A-2 8:1723-1740, 1970.

[3] G. Meinel and A. Peterlin. Plastic deformation of polyethylene. II. Change of mechanical properties during drawing. Journal of Polymer Science, Part A-2 9:67-83, 1971.

[4] G. Meinel and A. Peterlin. Plastic deformation of polyethylene. III. Mechanical properties and morphology of drawn low density polyethylene. European Polymer Journal 7:657-670, 1971.

[5] P.B. Bowden and R.J. Young. Deformation mechanisms in crystalline polymers. Journal of Materials Science 9:2034-2051, 1974.

[6] S.G. Burnay and G.W. Groves. Deformation mechanisms in oriented high density polyethylene. Journal of Materials Science 13:639-646, 1978.

[7] N.S.J.A. Gerrits and Y. Tervoort. Deformation mechanisms during uniaxial drawing of meltcrystallized ultra-high molecular weight polyethylene. Journal of Material Science 25:13851390, 1992.

[8] Z. Bartczak, R.E. Cohen, and A.S. Argon. Evolution of the crystalline texture of high density polyethylene during uniaxial compression. Macromolecules 25:4692-4704, 1992.

[9] Z. Bartczak, A.S. Argon, and R.E. Cohen. Deformation mechanisms and plastic resistence in single crystal textured high density polyethylene. Macromolecules 25:5036-5053, 1992.

[10] Z. Bartczak, A.S. Argon, and R.E. Cohen. Texture evolution in large strain simple shear deformation of high density polyethylene. Polymer 35:3427-3441, 1994.

[11] R. Hiss, S. Hobeika, C. Lynn, and G. Strobl. Network stretching, slip processes and fragmentation of crystallites during uniaxial drawing of polyethylene and related copolymers. A comparative study. Macromolecules 32:4390-4403, 1999.

[12] D. Raabe, N. Chen, and L. Chen. Crystallographic texture, amorphization, and recrystallization in rolled and heat treated polyethylene terephthalate (PET). Polymer 45:8265-8277, 2004.

[13] J. Jia and D. Raabe. Evolution of crystallinity and of crystallographic orientation in isotactic polypropylene during rolling and heat treatment. European Polymer Journal 42:1755-1766, 2006.

[14] A. Peterlin. Molecular model of drawing polyethylene and polypropylene. Journal of Material Science 6:490-508, 1971.

[15] R. Popli and L. Mandelkern. Influence of structural and morphological factors on the mechanical properties of the polyethylenes. Journal of Polymer Science, Part B: Polymer Physics 25:441-483, 1987.

[16] R.J. Young. A dislocation model for yield in polyethylene. Philosophical Magazine 30, 1974.

[17] R.J. Young. Screw dislocation model for yield in polyethylene. Materials forum 11:210-216, 1988. 
[18] R. Séguéla, V. Gaucher-Miri, and S. Elkoun. Plastic deformation of polyethylene and ethylene copolymers: Part I. Homogeneous crystal slip and molecular mobility. Journal of Material Science 33:1273-1279, 1998.

[19] R. Séguéla. Dislocation approach to the plastic deformation of semicrystalline polymers: kinetic aspects for polyethylene and polypropylene. Journal of Polymer Science, Part B: Polymer Physics 40:593-601, 2002.

[20] S. Nikolov and D. Raabe. Yielding of polyethylene through propagation of chain twist defects: temperature, stem length and strain-rate dependence. Polymer 47:1696-1703, 2006.

[21] D.M. Parks and S. Ahzi. Polycrystalline plastic deformation and texture evolution for crystals lacking five independent slip systems. Journal of the Mechanics and Physics of Solids 38:701-724, 1990.

[22] B.J. Lee, D.M. Parks, and S. Ahzi. Micromechanical modeling of large plastic deformation and texture evolution in semicrystalline polymers. Journal of the Mechanics and Physics of Solids 41:1651-1687, 1993.

[23] B.J. Lee, A.S. Argon, D.M. Parks, S. Ahzi, and Z. Bartczak. Simulation of large strain plastic deformation and texture evolution in high density polyethylene. Polymer 34:3555-3575, 1993.

[24] B.J. Lee, S. Ahzi, and R.J. Asaro. On the plasticity of low symmetry crystals lacking 5 independent slip systems. Mechanics of Materials 20:1-8, 1995.

[25] J.A.W. van Dommelen, D.M. Parks, M.C. Boyce, W.A.M Brekelmans, and F.P.T Baaijens. Micromechanical modeling of the elasto-viscoplastic behavior of semi-crystalline polymers. Journal of the Mechanics and Physics of Solids 51:519-541, 2003.

[26] J.A.W. van Dommelen, D.M. Parks, M.C. Boyce, W.A.M Brekelmans, and F.P.T Baaijens. Micromechanical modeling of intraspherulitic deformation of semicrystalline polymers. Polymer 44:6089-6101, 2003.

[27] J.A.W. van Dommelen, B.A.G. Schrauwen, L.C.A. van Breemen, and L.E. Govaert. Micromechanical modeling of the tensile behavior of oriented polyethylene. Journal of Polymer Science, Part B: Polymer Physics 42:2983-2994, 2004.

[28] S. Nikolov, R.A. Lebensohn, and D. Raabe. Self-consistent modeling of large plastic deformation, texture and morphology evolution in semi-crystalline polymers. Journal of the Mechanics and Physics of Solids 54:1350-1375, 2006.

[29] A. Sedighiamiri, T.B. van Erp, G.W.M. Peters, L.E. Govaert, and J.A.W. van Dommelen. Micromechanical modeling of the elastic properties of semicrystalline polymers: a threephase approach. Journal of Polymer Science, Part B: Polymer Physics 48:2173-2184, 2010.

[30] T Ree and H. Eyring. Theory of non-Newtonian flow. I. Solid plastic system. Journal of Applied Physics 26:793-800, 1955.

[31] T Ree and H. Eyring. Theory of non-Newtonian flow. II. Solution system of high polymers. Journal of Applied Physics 26:800-809, 1955.

[32] E.H. Lee. Elastic-plastic deformation at finite strains. Journal of Applied Mechanics: Transactions of the ASME 36:1-6, 1969.

[33] L. Lin and A.S. Argon. Structure and plastic deformation of polyethylene. Journal of Materials Science 29:294-323, 1994. 
[34] A. Galeski. Strength and toughness of crystalline polymer systems. Progress in Polymer Science 28:1643-1699, 2003.

[35] B. Crist, C.J. Fisher, and P.R. Howard. Mechanical properties of model polyethylenes: tensile elastic modulus and yield stress. Macromolecules 22:1709-1718, 1989.

[36] R.H. Boyd. Relaxation processes in crystalline polymers: molecular interpretation - a review. Polymer 26:1123-1133, 1985.

[37] K. Schmidt-Rohr and H.W. Spiess. Chain diffusion between crystalline and amorphous regions in polyethylene detected by 2D exchange carbon-13 NMR. Macromolecules 24:5288$5293,1991$.

[38] M.C. Boyce, D.M. Parks, and A.S. Argon. Large inelastic deformation of glassy polymers. part I: rate dependent constitutive model. Mechanics of Materials 7:15-33, 1988.

[39] E.M. Arruda and M.C. Boyce. A three-dimensional constitutive model for the large stretch behavior of rubber elastic materials. Journal of the Mechanics and Physics of Solids 41:389-412, 1993.

[40] M.C. Boyce, E.L. Montagut, and A.S. Argon. The effects of thermomechanical coupling on the cold drawing process of glassy polymers. Polymer Engineering and Science 32:1073-1085, 1992.

[41] C. G'Sell, S. Boni, and S. Shrivastava. Application of the plane simple shear test for determination of the plastic behaviour of solid polymers at large strains. Journal of Materials Science 18:903-918, 1983.

[42] K. Tashiro, M. Kobayashi, and H. Tadakoro. Calculation of the three-dimensional elastic constants of polymer crystals. 2. Application to orthorhombic polyethylene and poly(vinyl alcohol). Macromolecules 11:914-918, 1978.

[43] F. Bedoui, J. Diani, G. Regnier, and W. Seiler. Micromechanical modeling of isotropic elastic behavior of semicrystalline polymers. Acta Materialia 54:1513-1523, 2006.

[44] D.C. Basset and A.M. Hodge. On the morphology of melt-crystallized polyethylene I. Lamellar profiles. Proceedings of the Royal Society of London A 377:25-37, 1981.

[45] S. Gautam, S. Balijepalli, and G.C. Rutledge. Molecular simulations of the interlamellar phase in polymers: effect of chain tilt. Macromolecules 33:9136-9145, 2000.

[46] N.W. Brooks, R.A. Duckett, and I.M. Ward. Investigation into double yield points in polyethylene. Polymer 33:1872-1880, 1992.

[47] N.W.J. Brooks, A.P. Unwin, R.A. Duckett, and I.M. Ward. Double yield points in polyethylene: structural changes under tensile deformation. Journal of Macromolecular Science, Part B: Physics 34:29-54, 1995.

[48] N.W.J. Brooks, R.A. Duckett, and I.M. Ward. Modeling of double yield points in polyethylene: temperature and strain-rate dependence. Journal of Rheology 39:425-436, 1995.

[49] R. Séguéla and O. Darras. Phenomenological aspects of the double yield of polyethylene and related copolymers under tensile loading. Journal of Material Science 29:5342-5352, 1994.

[50] J.C. Lucas, M.D. Failla, F.L. Smith, and L. Mandelkern. The double yield in the tensile deformation of the polyethylenes. Polymer Engineering and Science 35:1117-1123, 1995.

[51] M.F. Butler, A.M. Donald, and A.J. Ryan. Time resolved simultaneous small- and wideangle X-ray scattering during polyethylene deformation: 1. Cold drawing of ethylene- $\alpha-$ olefin copolymers. Polymer 38:5521-5538, 1997. 
[52] M.F. Butler, A.M. Donald, and A.J. Ryan. Time resolved simultaneous small- and wide-angle X-ray scattering during polyethylene deformation - II. Cold drawing of linear polyethylene. Polymer 39:39-52, 1998.

[53] M.F. Butler, A.M. Donald, and A.J. Ryan. Time resolved simultaneous small- and wideangle X-ray scattering during polyethylene deformation 3. Compression of polyethylene. Polymer 39:781-792, 1998.

[54] V. Gaucher-Miri and R. Séguéla. Tensile yield of polyethylene and related copolymers: mechanical and structural evidences of two thermally activated processes. Macromolecules 30:1158-1167, 1997.

[55] R.P.M. Schrauwen, B.A.G.and Janssen, L.E. Govaert, and H.E.H. Meijer. Intrinsic deformation behavior of semicrystalline polymers. Macromolecules 37:6069-6078, 2004.

[56] H.G.H. van Melick, L.E. Govaert, and H.E.H. Meijer. Localisation phenomena in glassy polymers: influence of thermal and mechanical history. Polymer 44:3579-3591, 2003. 


\title{
Short- and long-term failure kinetics 1
}

Chapter 4

\begin{abstract}
The process of plastic deformation in semicrystalline polymers is complicated due to the operation of a variety of mechanisms at different levels and is strongly dependent on their underlying microstructure. The objective of this work is to establish a quantitative relation between the microstructure and the mechanical performance of semicrystalline polymers, as characterized by elasto-viscoplastic deformation. In order to do that, a micromechanically based constitutive model is used. The model describes the material as an aggregate of two-phase layered composite inclusions, consisting of crystalline lamellae and amorphous layers. The starting point for adding quantitative abilities to the model, in particular for the yield kinetics, is formed by experimental observations on both the yield kinetics and the time-to-failure of polyethylene at different temperatures, which reveal the contribution of two relaxation processes. To predict the thermo-rheologically complex short-term and long-term failure behavior, the crystallographic slip kinetics and the amorphous yield kinetics are reevaluated, and the Eyring flow rule is modified by adding a temperature shift function. To enable the prediction of both tension and compression, a non-Schmid effect is added to the constitutive relation of each slip system. The creep behavior of polyethylene is then simulated directly using the multi-scale, micromechanical model, predicting the time-to-failure, controlled by plastic deformation.
\end{abstract}

\footnotetext{
${ }^{1}$ Reproduced from: A. Sedighiamiri, L.E. Govaert, M.J.W. Kanters, J.A.W. van Dommelen, Micromechanics of semicrystalline polymers: Yield kinetics and long-term failure, Journal of Polymer Science Part B: Polymer Physics, accepted, 2012.
} 


\subsection{Introduction}

Plastic flow of semicrystalline polymers is a complex multi-level process, due to the complex hierarchical structure of these materials and various morphological and structural factors that play a role at different levels during deformation [112]. Any macroscopically homogenous deformation is accompanied by the operation of a variety of mechanisms in the heterogeneous microstructure. Therefore, the mechanical behavior of semicrystalline polymers strongly depends on the initial underlying microstructure, and the evolution of the microstructure during deformation. Much experimental and modeling studies [13-26] have been focused on understanding the elasto-viscoplastic behavior of semicrystalline polymers, specifically polyethylene, establishing a relation between the morphological features (crystallinity, lamellar thickness and molecular weight) and the macroscopic mechanical properties (yield stress, strain hardening and elastic modulus).

A first attempt to relate the crystalline structure of semicrystalline polyethylene to the yield stress, was made by Young [27, 28], who regarded the yield process to be controlled by the nucleation of dislocations. This approach assumes that yield involves the activation of [001] screw dislocations, accounting for a single crystallographic slip system, and enables a direct relation between macroscopic yield stress and lamellar thickness. Shadrake and Guiu [29] proposed that the energy required for nucleation of screw dislocations from the edge of the crystals, with a Burgers vector parallel to the chain direction, is supplied by thermal fluctuations of chain segments within crystals. This was later supported by Séguéla et al. [30, 31] who pointed out that slip occurs due to the nucleation of screw dislocations from the lateral surface of the crystalline lamellae and proposed that the underlying mechanism for the nucleation of screw dislocations relies on chain twist defects that migrate along the chain stems. Such conformational defects are also responsible for translational mobility of chain segments within the crystal, referred to as chain diffusion, and are required for the $\alpha$-relaxation process, resulting in reorientation and relaxation of the interlamellar material [32]. This approach was further expanded by Nikolov and Raabe [33], who modeled the stress-induced motion of the chain twist defects within the dislocation core, enabling them to analyze the temperature and strain rate dependence of yield, by directly relating the macroscopic strain rate $\dot{\epsilon}$ to the microscopic slip rate $\dot{\gamma}$.

Unfortunately, these approaches only account for a single slip system, and it is well known that single polymer crystals have more modes of deformation. For instance, the crystalline phase in HDPE employs eight physically distinct slip systems [7], which effectively comprise four independent modes of deformation. 
Moreover, such an approach does not account for the crystallographic and morphological texture evolutions during deformation, which have a great influence on the stress-strain response of semicrystalline polymers [34]. Parks and Ahzi [15] employed a viscoplastic Taylor-type model for a purely crystalline material in a polycrystalline approach to include all physically available slip systems, enabling them to qualitatively describe the texture development of HDPE during deformation. However, Taylor's assumption leads to the prediction of a high post-yield stress-strain response and overestimates the rate of texture evolution. The approach was significantly improved by formulating more sophisticated interaction laws and by the introduction of the amorphous phase. Lee et al. [18, 19] developed a specific micromechanical model in which a rigidviscoplastic two-phase composite inclusion model, consisting of mechanically coupled amorphous and crystalline phases, was used in combination with a Sachs-like and two hybrid interaction laws to predict the stress-strain behavior and crystallographic texture evolution of HDPE. The rigid-viscoplastic approach was further extended by Van Dommelen et al. [23, 24] to an elasto-viscoplastic framework for large deformation of semicrystalline polymers. In this model, the behavior of the amorphous phase was assumed to be isotropic elastic with a rate-dependent plastic flow with strain hardening resulting from molecular orientation. The crystalline phase was modeled as anisotropic elastic with plastic flow occurring via crystallographic slip. Recently, Sedighiamiri et al. [26] used a hybrid numerical/experimental procedure to add quantitative predictive abilities the micromechanical model of Van Dommelen et al. [23, 24] by characterization of the stress-dependence of the rate of plastic deformation, the slip kinetics. To quantitatively predict the deformation kinetics, the previously used power law relation [23] was replaced by an Eyring flow rule [35].

With most modeling studies focusing on the short-term deformation kinetics of semicrystalline materials, i.e. the response under applied constant strain rate, only a few phenomenological [36-38] and no morphological modeling studies on the long-term behavior and time-to-failure of these materials exist to the authors' knowledge. The time-scale at which polymeric materials fail depends strongly on the loading conditions. Originating from their molecular mobility, polymers always fail, even under constant static loads well below their yield stress, also referred to as creep deformation. Therefore, the possibility to predict yield and failure at different time-scales under designed load specifications, is a challenging task and an essential requirement for safe application of load-bearing polymeric materials. Due to their extensive range of load-bearing applications, the creep behavior of semicrystalline polymers, especially polyethylene, has been widely studied by experiments [39-42]. Experimental observations on long-term properties of isotropic semicrystalline polymers [41, 42] reveal that the time-to- 
failure under static loading conditions is controlled by three principal failure modes. At high stress levels and low temperatures, a ductile failure mode due to strain localization, is encountered. At lower stress levels, a brittle failure mode occurs, since the stress dependence of the time-to-failure is controlled by the kinetics of slow crack growth. Finally, at very low applied stress and very long lifetime, a nearly stress independent failure mode, associated with molecular degradation, is observed. Regardless of failure modes, the time-tofailure under long-term static loading increases with decreasing applied stress and temperature. However, in the absence of a quantitative model for long-term lifetime prediction, real-time time-to-failure experiments need to be performed, which are lengthy and impractical.

In the present work, it is attempted to provide a quantitative relation between the microstructure and the deformation kinetics (both short-term and long-term) of semicrystalline polymers at different deformation modes and temperatures. To do that, a multi-scale micromechanical model is employed [23, 26]. A key issue is the stress-dependence of the microscopic rate of plastic deformation, the slip kinetics, which is the mechanism underlying time-dependent, macroscopic failure. The kinetics of the macroscopic plastic flow is strongly dependent on the rate-dependence of slip along crystallographic planes, together with the yield kinetics of the amorphous domain. Therefore, an accurate quantitative prediction requires a proper description of the slip kinetics and amorphous yield kinetics. The starting point is formed by the experimental observations on the yield kinetics of polyethylene at different temperatures and strain rates, which reveal the contribution of two deformation processes. Further experimental data on the stress dependence of the time-to-failure show a piecewise linear relation in semi-logarithmic plots, with the same absolute slope as that of the yield kinetics. This indicates that the kinetics of failure under applied strain rate and applied stress are strongly related. To predict failure under both conditions and for different temperatures, the crystallographic slip kinetics and the amorphous yield kinetics are re-evaluated, and the Eyring flow rule is modified by adding a temperature shift function. To capture the influence of hydrostatic stress, e.g. the difference between tension and compression, a non-Schmid effect is added to the constitutive relation of each slip system. The creep behavior of polyethylene is then simulated directly using the multi-scale, micromechanical model, predicting the time-to-failure, controlled by plastic deformation (ductile failure), without any additional fitting parameter. 


\subsection{Experimental}

\subsubsection{Material}

The semicrystalline polymer used in this study is a polyethylene grade supplied by Sabic (Vestolen A 6060R), with a bimodal molecular weight distribution, which improves the long-term performance enabling application of the material in pressurized pipe systems for water, gas and sewage. This Vestolen grade is certified as a PE100 pipe grade.

\subsubsection{Sample preparation}

Sheet material of $1.5 \mathrm{~mm}$ and $8 \mathrm{~mm}$ thickness was compression molded in a hotpress at $230{ }^{\circ} \mathrm{C}$. The force was step-wise increased to $100 \mathrm{kN}$ and kept constant for 3 minutes. Subsequently, the mold was taken from the hot-press and allowed to cool in ambient temperature, which resulted in a cooling rate of about $5^{\circ} \mathrm{C} / \mathrm{min}$. The resulting degree of crystallinity of the material was measured by wide angle X-ray scattering experiments. For tensile experiments, dog-bone shaped samples, with dimensions of the smallest section of $55 \times 5 \mathrm{~mm}$, were punched from the 1.5 $\mathrm{mm}$ thick compression molded sheets. For uniaxial compression tests, cylindrical samples $(\varnothing 6 \times 6 \mathrm{~mm})$ were machined from the $8 \mathrm{~mm}$ thick plates.

\subsubsection{Mechanical testing}

Both tensile and compression tests were performed at constant, linear strain rates varying from $10^{-5} \mathrm{~s}^{-1}$ up to $10^{-1} \mathrm{~s}^{-1}$ at different temperatures. Long-term failure experiments were performed under load control, applying a constant load in uniaxial tension during which the displacement was recorded. The point of failure is found by taking the maximum strain rate when observing the evolution of strain rate versus strain in a so called Sherby-Dorn plot [43]. The time needed to apply the constant load was negligible in comparison to the time-to-failure obtained. For all measurements, performed at different temperatures, the sample was allowed to warm to equilibrium temperature for 15 minutes before testing.

\subsubsection{Size exclusion chromatography (SEC)}

Hight temperature SEC analysis was performed on as-received and loaded samples using a Waters Alliance GPCV 2000 chromatograph equipped with three 
columns (three PL Olexis, $250 \times 8.5 \mathrm{~mm}$, Polymer Laboratories Ltd). Samples were eluted with trichlorobenzene at a flow rate of $1 \mathrm{~mL} \cdot \mathrm{min}^{-1}$ at $160{ }^{\circ} \mathrm{C}$.

\subsection{Constitutive modeling}

An aggregate of a discrete number of layered two-phase composite inclusions [18, 19] is used to represent the constitutive behavior of microscopically heterogeneous semicrystalline material at the macroscopic scale. Each composite inclusion is comprised of a crystalline lamella and an amorphous layer. These two phases are mechanically coupled by enforcing kinematical compatibility and traction equilibrium on the interface between the crystalline lamella and the amorphous layer. A microstructural elasto-viscoplastic constitutive model is used for both constituent phases. In this section, the kinematical framework and the constitutive models for the elastic and viscoplastic behavior of each phase together with the polycrystalline aggregate model are concisely summarized. The constitutive material models of each domain and the set of equations that compose the composite inclusion model are summarized in Appendix 4.A. A more detailed discussion of the models employed here, is given by Van Dommelen et al. [23].

\subsubsection{Kinematics}

For a microstructural elasto-viscoplastic constitutive description of the constituent phases, the deformation gradient tensor $\boldsymbol{F}=\left(\vec{\nabla}_{0} \vec{x}\right)^{T}$ of each phase is decomposed into a plastic and an elastic part, denoted by the subscripts " $p$ " and "e", respectively [44]:

$$
\boldsymbol{F}^{\pi}=\boldsymbol{F}_{\mathrm{e}}^{\pi} \cdot \boldsymbol{F}_{\mathrm{p}}^{\pi}, \quad \text { with } \quad \pi=\mathrm{a}, \mathrm{c},
$$

where the superscript $\pi$ shows either the amorphous or the crystalline phase. Subsequently, the constitutive behavior of both the crystalline and the amorphous phase is specified.

\subsubsection{Crystalline domain}

The crystalline domain of semicrystalline polyethylene consists of regularly ordered chain segments in an orthorhombic packing. This crystal structure causes the crystalline phase to possess highly anisotropic elastic properties with a high elastic modulus in the chain direction, and to have plastic deformation primarily 
governed by crystallographic slip up to moderate strains [5-7, 9]. Moreover, polymer crystals may also deform plastically by mechanical twinning and stressinduced martensitic phase transformation [5, 9, 45]. Since crystallographic slip is the predominant deformation mode at the initial stages of deformation and can accommodate larger plastic deformations than the latter two mechanisms, for the modeling purpose the other two mechanisms are left out of consideration. Crystallographic slip within the crystalline lamellae can take place by either fine slip or coarse slip [9]. During the early stages of deformation, fine slip, which changes the angle between the chain direction and the interface normal, is the predominant mode of plastic deformation, whereas coarse slip, which does not alter the angle between the chains and the interface normal and will ultimately lead to lamellar break-up, becomes more important at larger strains. In this study, all slips are assumed to be of the fine slip type.

The elastic part of the deformation in the crystalline domain is characterized by an anisotropic fourth order elasticity tensor, which linearly relates the GreenLagrange strain tensor and the elastic second Piola-Kirchhoff stress measure.

A rate-dependent crystal plasticity model is employed for the viscoplastic behavior of the crystalline phase. In this model, the plastic flow rate of the crystalline lamella, consisting of a single crystal, is described by the contributions of all $N_{S}$ physically distinct slip systems, being 8 for high density polyethylene, and is given by:

$$
\boldsymbol{L}_{\mathrm{p}}^{\mathrm{c}}=\dot{\boldsymbol{F}}_{\mathrm{p}}^{\mathrm{c}} \cdot \boldsymbol{F}_{\mathrm{p}}^{\mathrm{c}-1}=\sum_{\alpha=1}^{N_{\mathrm{s}}} \dot{\gamma}^{\alpha} \boldsymbol{P}_{0}^{\alpha} \quad ; \quad \boldsymbol{P}_{0}^{\alpha}=\vec{s}_{0}^{\alpha} \otimes \vec{n}_{0}^{\alpha},
$$

with $\boldsymbol{P}_{0}^{\alpha}$ the nonsymmetric Schmid tensor, defined by the dyadic product of the unit slip direction $\vec{s}_{0}^{\alpha}$ and the unit slip plane normal $\vec{n}_{0}^{\alpha}$ in the reference configuration. The shear rate $\dot{\gamma}^{\alpha}$ of each slip system $\alpha$ was previously assumed to be related to the corresponding resolved shear stress $\tau^{\alpha}$ via a viscoplastic power law relation. In order to achieve a better quantitative prediction, Sedighiamiri et al. [26] replaced the power law relation with an Eyring flow rule:

$$
\dot{\gamma}^{\alpha}=\dot{\gamma}_{0}^{\alpha} \sinh \left(\frac{\tau^{\alpha}}{\tau_{0}^{\alpha}}\right),
$$

with $\dot{\gamma}_{0}^{\alpha}$ and $\tau_{0}^{\alpha}$ the reference shear rate and the shear strength, respectively. Eyring [35] developed a theory to describe the relaxation process of viscous flow in liquids, and it can also be used to describe the yield behavior of amorphous polymers, as well as the microscopic plastic flow at the level of crystallographic slip systems in semicrystalline polymers. However, varying 
loading conditions and non-isothermal conditions can change the deformation behavior and complicate the description of the rate-dependency of slip along crystallographic planes. Therefore, a more complex viscoplastic rate-dependent relation than the one in equation (4.3) needs to be employed to capture these conditions and provide a better quantitative predictive ability for the model. To do that, here a modified Eyring flow rule is used:

$$
\dot{\gamma}^{\alpha}=\dot{\gamma}_{0}^{\alpha} \underbrace{\exp \left(\frac{-\Delta U^{\alpha}}{R T}\right)}_{\text {(I) }} \underbrace{\sinh \left(\frac{\tau^{\alpha}}{\tau_{0}^{\alpha}}\right)}_{\text {(II) }} \underbrace{\exp \left(\frac{\mu^{\alpha} \sigma_{\mathrm{n}}^{\alpha}}{\tau_{0}^{c}}\right)}_{\text {(III) }} .
$$

The temperature dependence of slip kinetics is captured in part (I), where $\Delta U^{\alpha}$ is the activation energy of the slip system, $R$ the universal gas constant and $T$ the absolute temperature. Part (II) represents the stress dependence of the plastic flow, and part (III) accounts for the effect of pressure on plastic flow in terms of the normal stress on each slip plane, with $\mu^{\alpha}$ the normal stress dependency coefficient and $\sigma_{\mathrm{n}}^{\alpha}$ the normal stress on the slip plane.

\subsubsection{Amorphous domain}

The amorphous domain of semicrystalline polymers consists of an assembly of randomly coiled macromolecules, strongly connected to their adjacent crystalline lamellae through tie molecules, loose chain folds, cilia and intercrystalline chains crossing the interface of the crystalline and amorphous phase. In the case of polyethylene, the glass transition temperature of the amorphous phase is far below room temperature. The amorphous phase should, therefore, be in the rubbery state at room temperature. However, there are some indications in literature [46, 47] that suggest that the interlamellar layers may be stiffer than purely bulk amorphous material due to the confinement of amorphous layers between thick crystalline lamellae, leading to the immobilization of amorphous segmental re-orientation.

Due to the strong connectivity of the constituent phases in semicrystalline polymers through many chains crossing the crystalline/amorphous interface, such as tight or loose chain ends, cilia, and intercrystalline links, the crystallographic mechanisms are inevitably accompanied by interlamellar deformation and they can only deform simultaneously [48]. Furthermore, random thermal fluctuations allow chain segments in the crystalline phase to migrate into the amorphous domain. Chain ends or loose chain folds can similarly escape from the amorphous phase into the crystalline lamellae. This process of chain diffusion [49], generally referred to as $\alpha$-relaxation, results in redistribution of the 
chain segments that constitute the interlamellar layer. Therefore, the relaxation of the interlamellar material in a rate-dependent process occurs as well at a rate controlled by translational mobility of chain segments within the crystals [32]. In line with that, also the amorphous phase is modeled as elasto-viscoplastic, with kinetics influenced by that of the crystalline phase.

In earlier work [23], a Boyce-Parks-Argon-like model [50] combined with a viscoplastic power law relation was employed to model the elasto-viscoplastic deformation of the amorphous domain. Here, in the same framework, the effective shear strain rate and the effective shear stress are related by a modified Eyring flow rule:

$$
\dot{\gamma}_{\mathrm{p}}^{\mathrm{a}}=\dot{\gamma}_{0}^{\mathrm{a}} \exp \left(\frac{-\Delta U^{\mathrm{a}}}{R T}\right) \sinh \left(\frac{\tau^{\mathrm{a}}}{a \tau_{0}^{\mathrm{a}}}\right) \exp \left(\frac{-\mu^{\mathrm{a}} p^{\mathrm{c}}}{b \tau_{0}^{\mathrm{a}}}\right),
$$

with $\dot{\gamma}_{0}^{\mathrm{a}}$ the reference shear rate, $\Delta U^{\mathrm{a}}$ the activation energy of the amorphous yield kinetics, $\tau_{0}^{\mathrm{a}}$ the shear strength, $\mu^{\mathrm{a}}$ the pressure dependency coefficient and $p^{c}$ the pressure within the crystalline domain. The latter choice is made due to the fact that the kinetics of the relaxation of the amorphous domain originates from that of the crystalline phase. $a$ and $b$ are two constants accounting for the difference in the definition of the effective shear stress of the amorphous phase and the resolved shear stress of the crystalline phase, and of the crystalline normal stress and pressure, respectively, making them comparable for a uniaxial stress state. In that respect, $a$ and $b$ are taken to be 1.15 and 0.76 , respectively. The plastic rate of stretching is defined by an associated flow rule [23]. The Arruda-Boyce eight-chain network rubber elasticity model [51] is used to account for orientation induced hardening [50]. For more details about the constitutive model of the amorphous phase, see Appendix 4.A.

\subsubsection{Composite inclusion model}

In the multi-scale micromechanical model, two different levels are distinguished, namely the microscopic and macroscopic scale. At the microscopic scale, a layered two-phase composite entity is used as a representative microstructural element, as proposed by Lee et al. [18, 19] for rigid-viscoplastic material behavior. Each separate composite inclusion consists of a crystalline lamella, mechanically coupled to its corresponding amorphous layer to form a lamellar morphology, which is characterized by the crystalline lattice orientation, also referred to as crystallographic texture, and the interface orientation, also known as morphological texture; see figure 4.1. The stress and deformation fields within each phase are assumed to be homogeneous; however, they may differ 


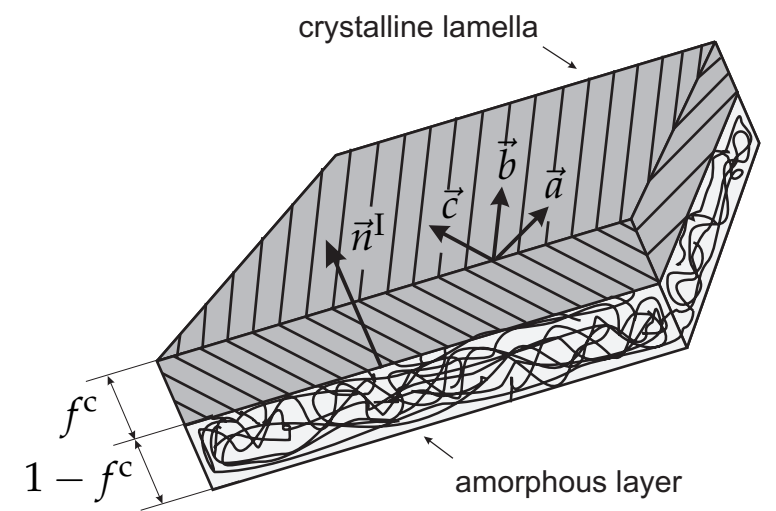

Figure 4.1 Schematic representation of a two-phase composite inclusion.

within each of the coupled phases. The inclusion-averaged deformation gradient and the inclusion-averaged Cauchy stress are given by the volume-average of the fields of the respective phases. Furthermore, kinematical compatibility and equilibrium conditions are defined on the interface of the crystalline lamella and the amorphous layer.

At the macroscopic level, the material is represented by an aggregate of composite inclusions. The mechanical response of the aggregate to the imposed deformation, is then computed by relating the volume-averaged deformation and stress fields of each layered composite inclusion to the corresponding fields of the aggregate using a hybrid local-global interaction law [18, 19, 23]. The consistency conditions for equilibrium and compatibility of the aggregate are maintained by enforcing the macroscopic stress and deformation to equal the volume-average of the stress and deformation fields of the composite inclusions.

In the hybrid interaction model, local-global compatibility conditions are assumed for the projections of the inclusion-averaged fields for which intrainclusion equilibrium conditions were formulated. Inversely, local-global equilibrium conditions are formulated for the components of the inclusion-averaged fields that are subjected to intra-inclusion compatibility. A more elaborate description of the composite inclusion model is presented in Appendix 4.A.

\subsubsection{Deformation modes}

Initially isotropic material is modeled by an aggregate of composite inclusions, represented by a set of random crystallographic orientations and corresponding lamellar orientations. The mechanical behavior of this aggregate is investigated for different deformation modes. For uniaxial tension and uniaxial compression, 
an aggregate of composite inclusions is subjected to a constant strain rate in the principal 1-direction:

$$
\overline{\boldsymbol{R}}=\boldsymbol{I} ; \quad \bar{U}_{11}=\lambda(t)
$$

with

$$
\begin{aligned}
& \lambda(t)=\exp (\dot{\epsilon} t) \quad \text { for tension, } \\
& \lambda(t)=\exp (-\dot{\epsilon} t) \quad \text { for compression, }
\end{aligned}
$$

where $\bar{R}$ is the macroscopic rotation tensor, and $\bar{U}$ is the corresponding right stretch tensor. Moreover, the components of the macroscopic Cauchy stress tensor $\bar{\sigma}$ should satisfy:

$$
\bar{\sigma}_{22}=\bar{\sigma}_{33}=\bar{\sigma}_{12}=\bar{\sigma}_{13}=\bar{\sigma}_{23}=0 \text {. }
$$

In the case of long-term static loading for creep failure, a constant nominal stress $\bar{P}$ is applied in the principal 1-direction of the material coordinate system. In line with that, the following conditions are imposed:

$$
\bar{P}=\bar{\sigma}_{11} \bar{J}\left\|\left[\left(\frac{\bar{J}}{J_{\Sigma}}\right)^{\frac{1}{3}} \sum_{i=1}^{N^{\mathrm{I}}} f_{0}^{\mathrm{I}^{i}} \boldsymbol{u}^{\mathrm{I}^{i}}\right]^{-\mathrm{T}} \cdot \vec{e}_{1}\right\|,
$$

with $\bar{\sigma}=\sum_{i=1}^{N^{\mathrm{I}}} f^{\mathrm{I}^{i}} \sigma^{\mathrm{I}^{i}}, \bar{J}=\sum_{i=1}^{N^{\mathrm{I}}} f_{0}^{\mathrm{I}^{i}} J^{\mathrm{I}^{i}}$ and $J_{\Sigma}=\operatorname{det}\left(\sum_{i=1}^{N^{\mathrm{I}}} f_{0}^{\mathrm{I}^{i}} \boldsymbol{F}^{\mathrm{I}^{i}}\right), f_{0}^{\mathrm{I}^{i}}$ the initial volume fraction of the $i$ th inclusion, and

$$
\bar{\sigma}_{22}=\bar{\sigma}_{33}=\bar{\sigma}_{12}=\bar{\sigma}_{13}=\bar{\sigma}_{23}=0 \text {. }
$$

\subsection{Phenomenology of failure}

In order to predict the mechanical response of semicrystalline polymers, first it is needed to further investigate their so-called short-term and long-term mechanical properties. Figure 4.2 a illustrates the short-term performance of HDPE, measured in a tensile test with a constant strain rate. The material displays an initially linear elastic region, where the stress increases linearly with strain. At higher stresses, the response becomes nonlinear and eventually reaches a maximum, the so-called engineering yield stress, which marks the onset of plastic deformation. Soon after, the material experiences necking due to strain localization [52] and a 
localized plastic zone is formed, which can propagate along the entire length of the test bar. From a mechanical point of view, the moment of neck initiation is regarded as the point of failure, i.e. where the material loses its integrity.

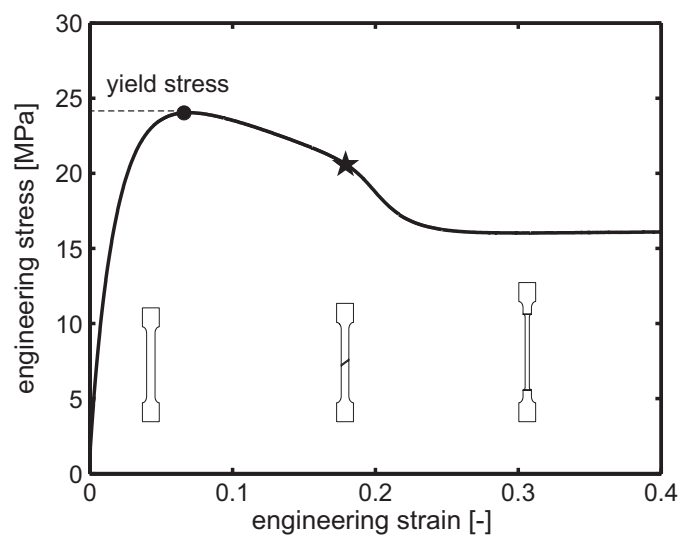

a

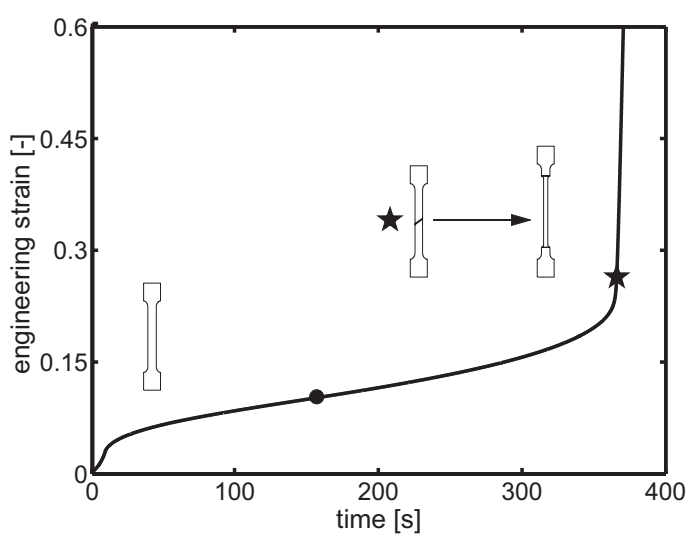

b

Figure 4.2 (a) Deformation behavior of isotropic HDPE in uniaxial tension under a constant strain rate of $0.001 \mathrm{~s}^{-1}$. (b) Creep behavior of the same material under a constant applied stress of $20 \mathrm{MPa}$.

Figure 4.2 b shows the long-term performance of the material, in which the evolution of the engineering strain under a constant applied stress that is approximately $16 \%$ below the yield stress, is illustrated. As can be seen, the deformation increases slowly in time, with a rate of deformation becoming constant (flow regime) after an initial viscoelastic region (primary creep). However, the rate of deformation abruptly increases at longer loading times, resulting in the failure of the material. The observed mode of failure is similar to that observed in shortterm performance, i.e. necking. The key issue, here, is that the applied stress dependency of the steady-state creep rate at the flow regime is interchangeable with the strain rate dependency of the yield stress.

Furthermore, the strain rate dependence of the yield stress and the applied stress dependence of the time-to-failure, as shown in figure 4.3 , both display a piecewise linear relation in semi-logarithmic plots with a similar absolute slope. This indicates that the kinetics of failure under applied strain rate and applied stress are strongly related.

The bilinear dependence of yield stress on strain rates implies the contribution two flow processes, and has been also observed in literature for semicrystalline polymers [53, 54]. The first flow process, which has a very low slope, acts at high temperatures or low strain rates, whereas the contribution of second process, with a higher slope, is revealed at low temperatures or high strain rates. 


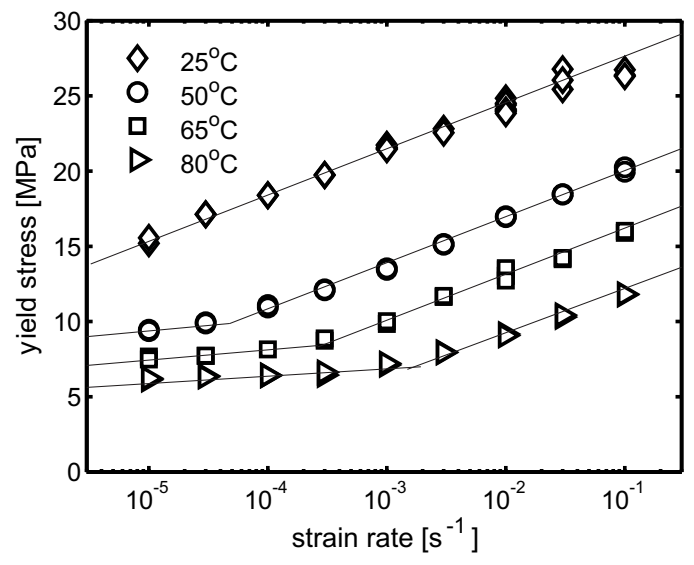

a

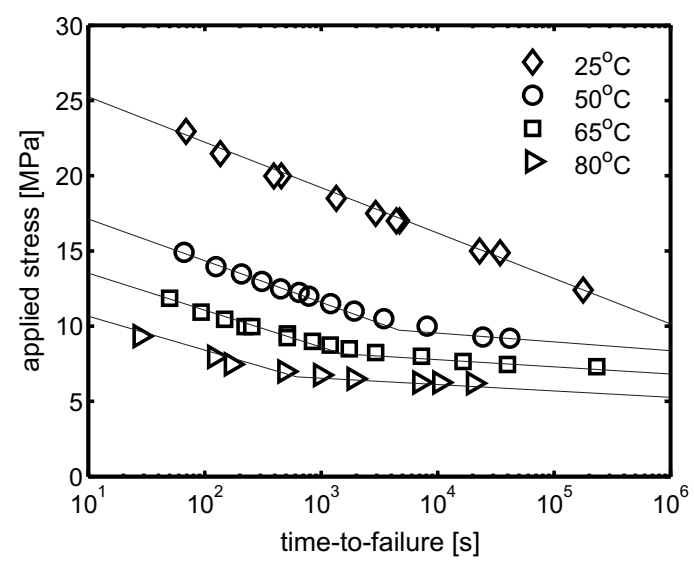

b

Figure 4.3 (a) Strain rate dependence of the yield stress, and (b) applied stress dependence of the time-to-failure of PE samples at different temperatures. Lines are a guide to the eye.

\subsection{Results and discussion}

In this section, the mechanical response of initially isotropic HDPE subjected to uniaxial tension and compression for a range of strain rates and temperatures is investigated. A volume crystallinity of $f^{c}=0.55$ is used in the model prediction. Experimental studies of melt-crystalized PE and molecular models show that lamellar surfaces are of the $\{h 0 l\}$ type, where the angle between the chain direction $\vec{c}$ and the lamellar normal direction $\vec{n}^{I}$ varies between $20^{\circ}$ and $40^{\circ}$ [55, 56]. Here, the lamellar surface is set to $\{201\}$, which corresponds with an angle of $35^{\circ}$. The spherulitic structure of melt-crystallized PE is represented by an aggregate of 500 randomly oriented inclusions.

It is now well established that the kinetics of the macroscopic plastic deformation in semicrystalline polymers are mainly governed by the kinetics of the individual slip systems, accompanied by the deformation kinetics of the amorphous domain at the microscopic level. Therefore, an accurate quantitative prediction of the yield kinetics and time-dependent macroscopic failure strongly depends on the proper description of the rate-dependency of slip along crystallographic planes together with the rate-dependency of the amorphous material. As a first step in adding quantitative predictive abilities to the multi-scale micromechanical model, the viscoplastic power law relation, often used in micromechanical modeling of polymers, was replaced with an Eyring flow rule [26]. The necessity of using a viscoplastic behavior for the amorphous phase was also discussed. The predicted compressive stress-strain behavior and the texture development during deformation were confronted with experimental data at room temperature, and 
revealed a promising agreement.

However, experimental data of the tensile yield kinetics of polyethylene at different temperatures and strain rates reveals the contribution of two relaxation processes, depicted as a clear change in slope of the strain rate dependence of the yield stress and the stress dependence of the time-to-failure, see figure 4.3 . In principle, one can distinguish two flow processes acting in a semicrystalline polymer, namely an intralamellar process related to crystallographic slip within the crystalline lamella, and interlamellar shear associated with the $\alpha$-relaxation process [57]. Depending on the temperature and time scale of the experiment, each or both of these processes may contribute to the mechanical response. At high temperatures or low strain rates, the stress contribution of the interlamellar process (process II) becomes negligible, and the yield kinetics of isotropic HDPE is governed by the intralamellar slip process (process I). For HDPE at a frequency of $1 \mathrm{~Hz}$, the $\alpha$-relaxation peak occurs at a temperature of approximately $80^{\circ} \mathrm{C}$ [47]. This implies that at higher temperatures and longer time scales, the interlamellar deformation associated with the $\alpha$-relaxation process can be easily activated, and no significant stress is needed to activate this process. At higher strain rates or lower temperatures, the additional contribution of the interlamellar process becomes apparent, resulting in a clear change of slope in the strain rate dependence of the yield stress [58, 59]. Figure 4.4 illustrates a schematic illustration of the molecular processes contributing to the mechanical response, with their stress contributions being additive. In order to describe this complex

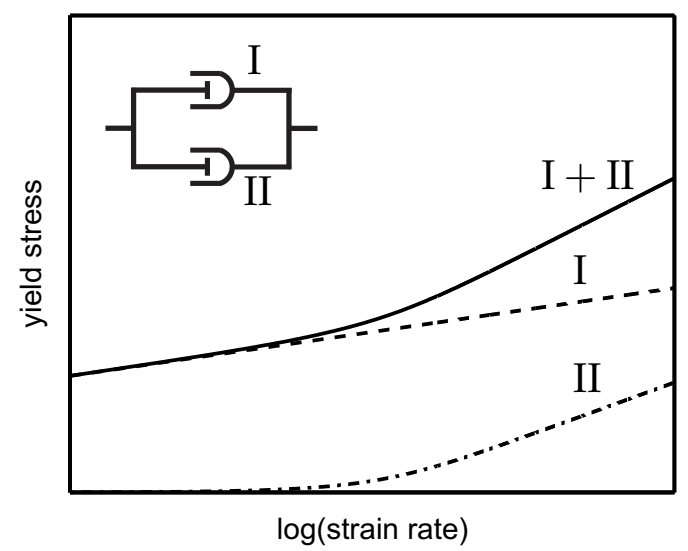

Figure 4.4 Schematic illustration of the stress contribution of two relaxation mechanisms on the yield kinetics.

behavior, the original Eyring rate expression for the individual slip kinetics and the kinetics of the amorphous domain is further modified. As a first step, an Arrhenius-type temperature shift function is added to capture the temperature 
dependency of the crystallographic slip and amorphous yield kinetics. In order to predict the response in both tension and compression, a pressure dependence of the yield kinetics of the amorphous material and a normal stress dependence of the crystallographic slip kinetics, also referred to as a non-Schmid effect, are included, as described in section 4.3. There are eight physically distinct slip systems present in the crystalline domain of polyethylene, which comprise six crystallographically different types of slip systems, each possibly having different properties. However, it should be noted that it is not possible to make a complete distinction between all different slip kinetics from experimental data on isotropic samples only, and various sets of parameters at the microscopic level could lead to a similar result at the macroscopic level. An illustrative example is given in figure 4.5. Crystallographic slip and amorphous yield kinetics at the microscopic level are classified into three distinct groups, namely the (100)[001] chain slip system as the easiest one, the amorphous yield kinetics, and the other slip systems. For the sake of simplicity, the pressure and non-Schmid effects are left out of consideration in this example. It is first attempted to mimic the thermo-rheologically complex yield kinetics (figure 4.3a), by assuming less rate sensitive slip kinetics and steeper amorphous yield kinetics, as can been in figure $4.5 \mathrm{a}$, since the first and the second relaxation mechanisms are assigned to the crystalline and amorphous domains, respectively. However, as can be seen in figure 4.5k, this set of kinetics does not result in a change in slope of the strain rate dependence of the yield stress, and provides a linear relation between the yield stress and the logarithm of the strain rate. Furthermore, figures $4.5 \mathrm{~b}$ and $4.5 \mathrm{c}$ reveal that using a different set of parameters, and parallel kinetics, still enables predicting a similar macroscopic deformation kinetics. In line with this, parallel slip kinetics are assumed for further characterization.

It has been shown by several researchers [53, 59.61] in their phenomenological models for the deformation kinetics of polymers, that using the Ree-Eyring [62] modification of the original Eyring expression enables describing the thermorheologically complicated behavior. The modified theory assumes that two independent flow processes exist, with the stress contributions being additive. Here, the same assumption is employed for the stress dependence of the crystallographic slip systems, and the yield kinetics of the amorphous phase. The resolved shear stress along the slip planes and the total contribution of the amorphous material, can, then, be expressed as:

$$
\tau^{\mathrm{tot}}=\tau^{\mathrm{I}}+\tau^{\mathrm{II}}
$$

Here, the transition in kinetics is incorporated in a single viscosity expression through extending the viscosity function for two parallel process. A derivation of the viscosity function and the corresponding parameters is given in 


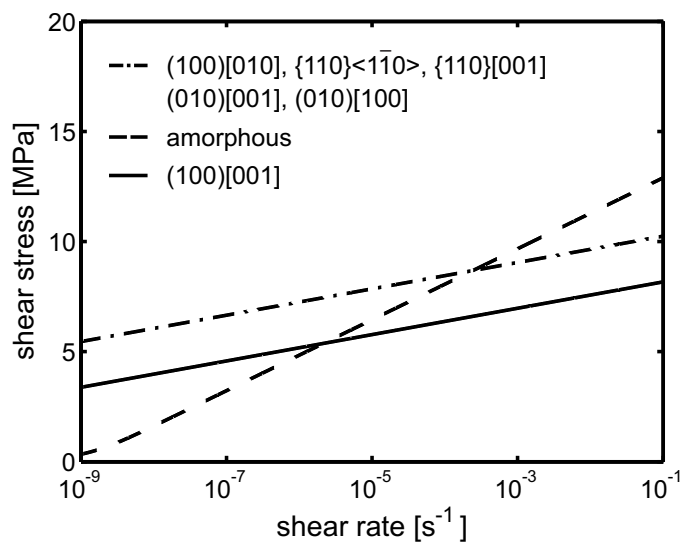

a

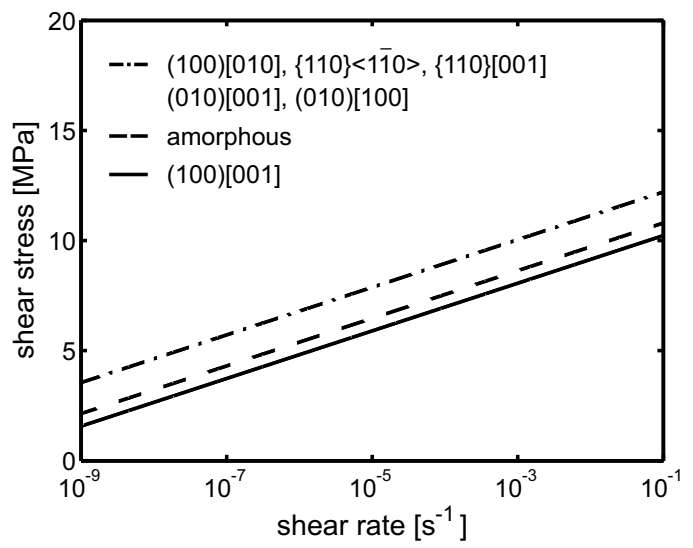

b

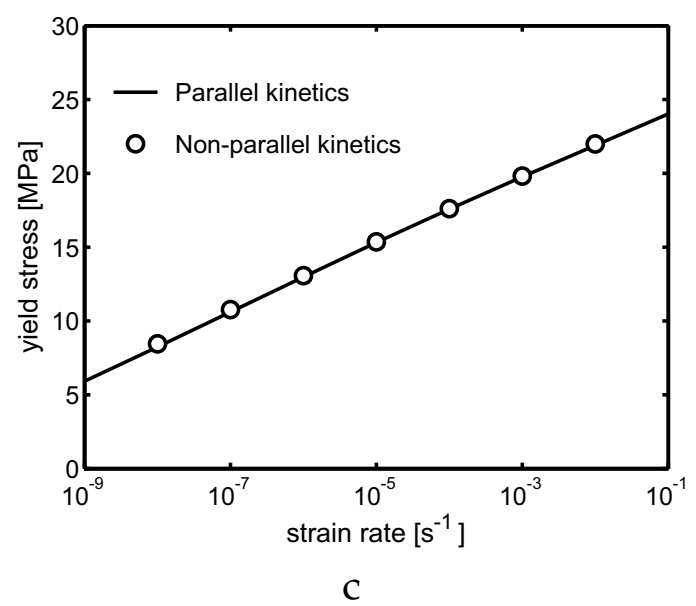

Figure 4.5 (a) Non-parallel slip and amorphous yield kinetics, (b) parallel slip and amorphous yield kinetics, and (c) their corresponding predicted macroscopic plastic flow in uniaxial tension.

\section{Appendix 4.B.}

As discussed in section 4.4, Experimental observations reveal that there is a similar failure mode in both applied strain rate and applied stress conditions. Ductile failure under an applied strain rate occurs due to strain localization associated with a local maximum in engineering stress. The sudden increase in creep rate under applied stress, which results in failure, is also due to necking of the material and the associated increase in local stress. Therefore, in order to predict failure under both conditions and for different temperatures, the crystallographic slip kinetics and the amorphous yield kinetics need to be further refined to predict the local maximum in engineering stress. 
The tensile stress-strain curves of isotropic HDPE, at various temperatures and strain rates, is shown in figure 4.6a. An important factor for accurate prediction

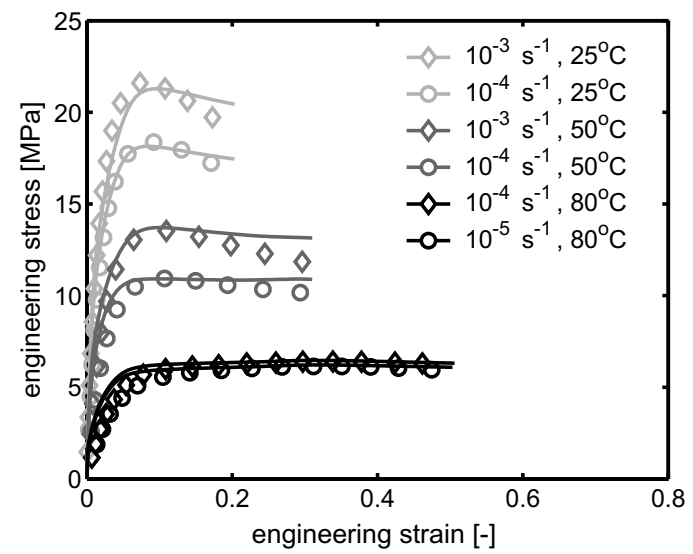

a

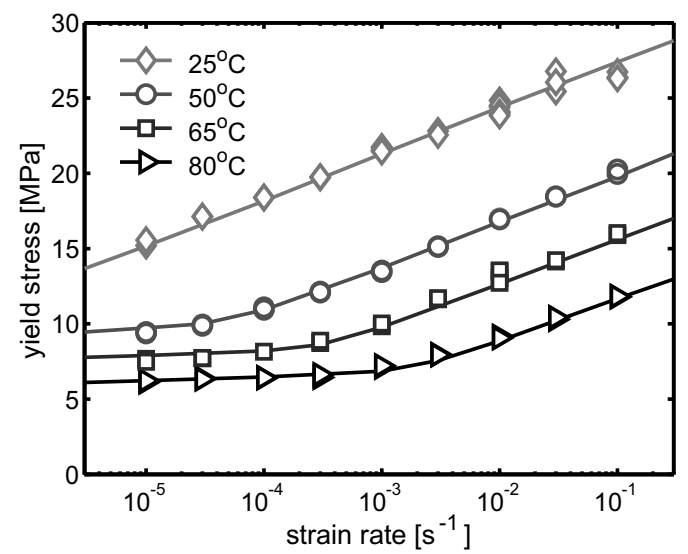

$\mathrm{b}$

Figure 4.6 (a) Tensile response of isotropic HDPE, at various temperatures and strain rates. (b) Temperature dependence of the tensile yield kinetics of isotropic HDPE. Lines show the micromechanical model prediction and symbols indicate experimental data.

of the tensile engineering stress-strain curves is the strain rate and temperature dependent elastic modulus of the material. A strain rate and temperature dependence of the elastic moduli of the constituent phases is not explicitly described in the model. However, the optimum amorphous modulus leading to the best description of the experimental tensile stress-strain curves at a specific strain rate, is determined for two temperatures and is taken to change linearly with temperature:

$$
G^{\mathrm{a}}=85-0.8 T
$$

with $G^{\mathrm{a}}[\mathrm{MPa}]$ the shear modulus of the interlamellar layer, and $T\left[{ }^{\circ} \mathrm{C}\right]$ the temperature. The bulk modulus of the interlamellar layer is chosen to be 3000 $\mathrm{MPa}$ [63]. Furthermore, in the Arruda-Boyce eight-chain model [51], accounting for the orientational hardening of the amorphous phase, the number of rigid links between entanglements and the constant proportional to the initial stiffness, are taken as $N=49$ and $\mu_{\mathrm{R}}=1 \mathrm{MPa}$, respectively. It was observed that changing the elastic constant of the crystalline phase with temperature does not have much effect on the tensile yield strain. Therefore, only the elastic modulus of the interlamellar layer is assumed to change with temperature. The elastic constants, used in this model for the crystalline phase, are listed in table 4.1.

The kinetics were first characterized for two extreme temperatures, namely $25^{\circ} \mathrm{C}$ 
Table 4.1 Elastic constants [GPa] for the crystalline phase of PE [64].

\begin{tabular}{ccccccccc}
\hline$C_{11}$ & $C_{22}$ & $C_{33}$ & $C_{12}$ & $C_{13}$ & $C_{23}$ & $C_{44}$ & $C_{55}$ & $C_{66}$ \\
\hline 7.99 & 9.92 & 315.92 & 3.28 & 1.13 & 2.14 & 3.62 & 1.62 & 3.19 \\
\hline
\end{tabular}

and $80^{\circ} \mathrm{C}$, to predict the local maximum in engineering stress, which could either be related to the first yield point, at low temperatures and high strain rates, or the second yield point, at high temperatures and low strain rates [16]. Figure 4.7 shows the obtained slip kinetics and the amorphous yield kinetics for these two temperatures. The re-evaluation of the slip kinetics was performed, taking into account both uniaxial tensile and compression data of isotropic HDPE. As can be seen, the kinetics of each slip system together with the kinetics of the amorphous phase include both processes, observed in the experimental data. The resulting

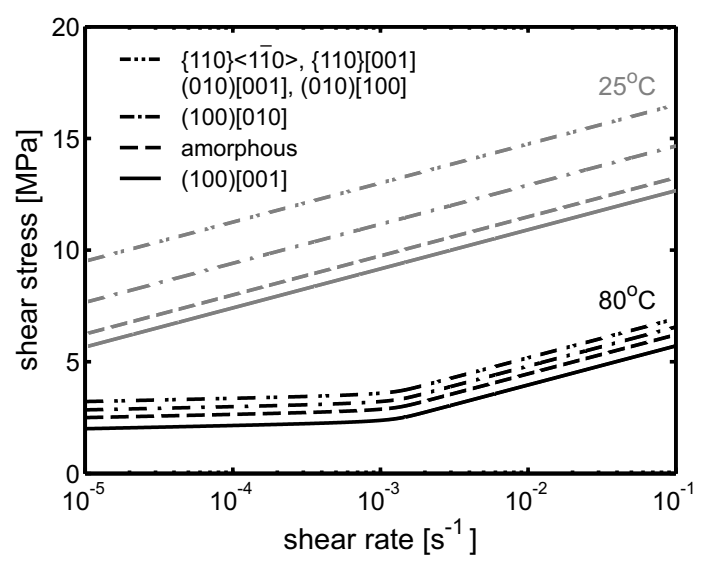

Figure 4.7 Refined slip kinetics and amorphous yield kinetics $\left(\frac{\tau^{\mathrm{a}}}{a}\right)$ at different temperatures.

micromechanical model description of the tensile engineering stress-strain curves at these two temperatures is illustrated in figure 4.6a. Then, the activation energies of the individual slip systems and the amorphous yield kinetics were calculated, enabling the prediction of the local engineering maximum at any temperatures in between. Table 4.2 summarizes the yield kinetics of all slip systems and the amorphous phase, together with their activation energies and the reference shear rates $\dot{\gamma}_{0, T_{\text {ref }}}^{*}$, characterized at a reference temperature $T_{\text {ref, }}$ for both relaxation processes. Since all slip kinetics and the amorphous yield kinetics account for the onset of the stress contribution of the interlamellar process at the same shear rate, the activation energies of the kinetics in process II are the same. 
The temperature dependence of the reference shear rates can then be calculated using:

$$
\dot{\gamma}_{0, T}^{*}=\dot{\gamma}_{0, T_{\text {ref }}}^{*} \exp \left[\frac{-\Delta U}{R}\left(\frac{1}{T}-\frac{1}{T_{\text {ref }}}\right)\right]
$$

with $T_{\text {ref }}$ being $80^{\circ} \mathrm{C}$ in this study. The values of the characteristic shear stresses for the relaxation processes within the crystalline and amorphous phases in the Eyring model are set to $\tau_{0}^{\mathrm{c}, \mathrm{I}}=\tau_{0}^{\mathrm{a}, \mathrm{I}}=0.06 \mathrm{MPa}$ and $\tau_{0}^{\mathrm{c}, \mathrm{II}}=\tau_{0}^{\mathrm{a}, \mathrm{II}}=0.7 \mathrm{MPa}$.

Table 4.2 Yield mechanisms in the constituent phases, with their reference shear rates and activation energies of both processes, corresponding to the viscoplastic Eyring flow rule, at $T_{\text {ref }}=80^{\circ} \mathrm{C}$.

\begin{tabular}{lcccc}
\hline & \multicolumn{2}{c}{ Process I } & \multicolumn{2}{c}{ Process II } \\
\hline Mechanism & $\dot{\gamma}_{0, T_{\text {ref }}}^{*}\left[\mathrm{~s}^{-1}\right]$ & $\Delta U^{\mathrm{I}}\left[\mathrm{kJ} \mathrm{mol}^{-1}\right]$ & $\dot{\gamma}_{0, T_{\text {ref }}}^{*}\left[\mathrm{~s}^{-1}\right]$ & $\Delta U^{\mathrm{II}}\left[\mathrm{kJ} \mathrm{mol}^{-1}\right]$ \\
\hline$(100)[001]$ & $6.5 \times 10^{-20}$ & 560 & $2.2 \times 10^{-3}$ & 110 \\
$(010)[001]$ & $1 \times 10^{-27}$ & 1293 & $2.2 \times 10^{-3}$ & 110 \\
$\{110\}[001]$ & $1 \times 10^{-27}$ & 1293 & $2.2 \times 10^{-3}$ & 110 \\
\hline$(100)[010]$ & $5 \times 10^{-26}$ & 867 & $2.2 \times 10^{-3}$ & 110 \\
$(010)[100]$ & $1 \times 10^{-27}$ & 1293 & $2.2 \times 10^{-3}$ & 110 \\
$\{110\}\langle 1 \overline{10}\rangle$ & $1 \times 10^{-27}$ & 1293 & $2.2 \times 10^{-3}$ & 110 \\
\hline amorphous & $1.6 \times 10^{-23}$ & 582 & $2.2 \times 10^{-3}$ & 110 \\
\hline
\end{tabular}

As can be seen in table 4.2, a large temperature dependence, corresponding to high activation energies of $867 \mathrm{~kJ} \mathrm{~mol}^{-1}$ and $1293 \mathrm{~kJ} \mathrm{~mol}^{-1}$, is observed for some slip systems in process I. These activation energies appear unrealistic for a plastic deformation process, since they are larger than the energy required for chain scission (breaking of a covalent C-C bond), which is in the range of $284-368 \mathrm{~kJ} \mathrm{~mol}^{-1}$ [65]. This indicates that chain scission could contribute to the deformation at elevated temperatures. To investigate this issue, molecular weight distribution was measured using SEC on as-received samples and samples loaded for various times (failure after 12.5 hours and interrupted after 7 hours loading) at $80^{\circ} \mathrm{C}$. The results are presented in table 4.3, where it becomes clear that there is no significant difference between molecular weights of three 
Table 4.3 Molecular weights of samples, as-received and loaded at $80^{\circ} \mathrm{C}$.

\begin{tabular}{cccc}
\hline samples & $\mathrm{M}_{\mathrm{n}}\left[\mathrm{kg} \mathrm{mol}^{-1}\right]$ & $\mathrm{M}_{\mathrm{w}}\left[\mathrm{kg} \mathrm{mol}^{-1}\right]$ & $\mathrm{M}_{\mathrm{z}}\left[\mathrm{kg} \mathrm{mol}^{-1}\right]$ \\
\hline as-received & 9.5 & 260 & 1300 \\
loaded for 7 hrs & 9.6 & 290 & 1500 \\
failed after $12.5 \mathrm{hrs}$ & 9.6 & 260 & 1200 \\
\hline
\end{tabular}

samples. Therefore, the chain scission can be excluded as a major contributor to the plastic deformation. This is completely in line with experimental data on pressurized pipe testing, where molecular degradation was never observed in the ductile failure regime [41]. Séguéla et al. [30, 31] proposed that the underlying mechanism for the nucleation and propagation of screw dislocations relies on defects that migrate along the chain stems within the crystalline domain. The change in the mobility of such a defect with temperature is captured by an Arrhenius temperature dependence. However, it should be noted that the defect density is also subject to change with temperature [66], and thus leads to a further increase in crystallographic slip rate. Therefore, it is hypothesized that the observed high activation energies are related to the collective effect of thermal activation of defect mobility and increase in defect density.

Figure 4.6a shows the prediction of the tensile stress-strain curves of isotropic HDPE with the micromechanical model, using the refined kinetics, at various strain rates and also an intermediate temperature, compared to the experimental data. The resulting temperature dependence of the tensile yield kinetics under constant strain rate is given in figure $4.6 \mathrm{~b}$.

The normal stress dependency coefficients of the slip systems and the pressure dependency coefficient of the amorphous phase were chosen to both account for the relative difference between the yield stress in tension and compression, and also the initial strain hardening, influencing the engineering tensile maximum. Table 4.4 lists the normal stress dependency coefficients along slip planes and the pressure dependency coefficient of the amorphous phase. Since specific data regarding the pressure dependence of the processes I and II are lacking, the normal stress and pressure dependence of the two processes are assumed to be equal.

The compressive true stress-strain response is shown in figure 4.8 $\mathrm{a}$. The initial post-yield hardening is partly due to texture evolution during deformation. However the initial hardening of the amorphous phase also affects the postyield response, especially up to moderate strains. By using a constant hardening 
Table 4.4 Amorphous phase pressure dependency and normal stress dependency coefficients of individual slip planes.

\begin{tabular}{cc}
\hline slip planes & $\mu$ \\
\hline$(100)$ & 0.005 \\
$(010)$ & 0.16 \\
$\{110\}$ & 0.16 \\
\hline amorphous & 0.004 \\
\hline
\end{tabular}

modulus $\mu_{\mathrm{R}}$ for the amorphous phase, the post-yield hardening in compression is insufficient. Orientational hardening of the amorphous phase is modeled by an Arruda-Boyce eight chain network model [51], which assumes a purely entropic origin for the strain hardening. However there are many arguments in contrast to such an hypothesis [67-70]. Van Melick et al. [67] showed that the strain hardening in amorphous glassy polymers decreases with increasing temperatures, whereas, in case of an entropic response, an increasing modulus would be expected. In addition, experimental evidence of the strain rate dependency of strain hardening for a selection of polymers is given in [69, 70]. Studies on the effect of hydrostatic pressure on the compressive deformation behavior of both amorphous and semicrystalline polymers have also revealed an increase of the strain hardening with increasing pressure [71, 72]. These observations suggest that in addition to the polymer chain network contribution, there is a relation between strain hardening and flow stress, i.e. a viscous contribution to strain hardening [73]. A physical picture would be that the thermal mobility of the chain segments in the amorphous region is influenced by inter-chain packing. When the interlamellar material is under compression, the free volume decreases, and therefore, the inter-chain Van der Waals forces increase, resulting in intensification of activation barriers for initiation of molecular mobility [74]. In correspondence to that, and to obtain a reasonable compressive stress-strain behavior, the hardening constant, proportional to the initial stiffness, for compressive deformation, is taken to be $\mu_{\mathrm{R}}=1.6 \mathrm{MPa}$.

Confrontation of true stress-strain curves predicted with the model for varying temperatures and strain rates with experimental data (figure 4.8 a) reveals a good agreement up to moderate strains. However, at later stages of deformation, specifically after the second yield point, the predictions deviate from the experimental curves. It is most likely due to the fact that lamellar fragmentation, as a result of a process of coarse slip [75-78], is not taken into account in the 


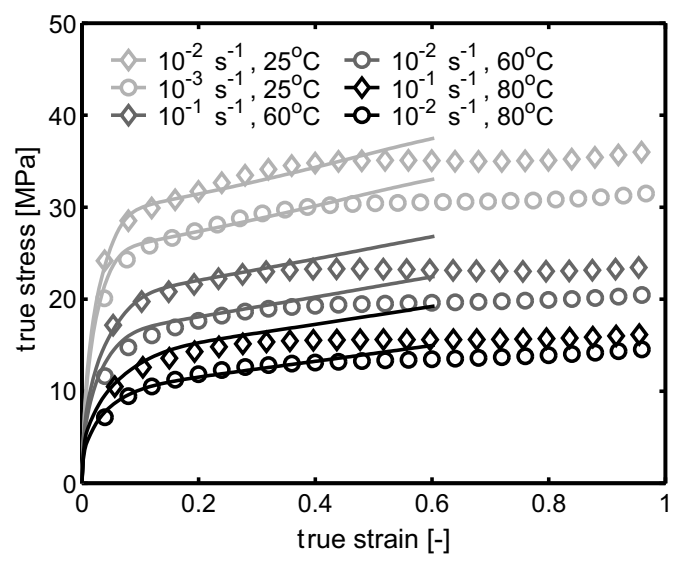

a

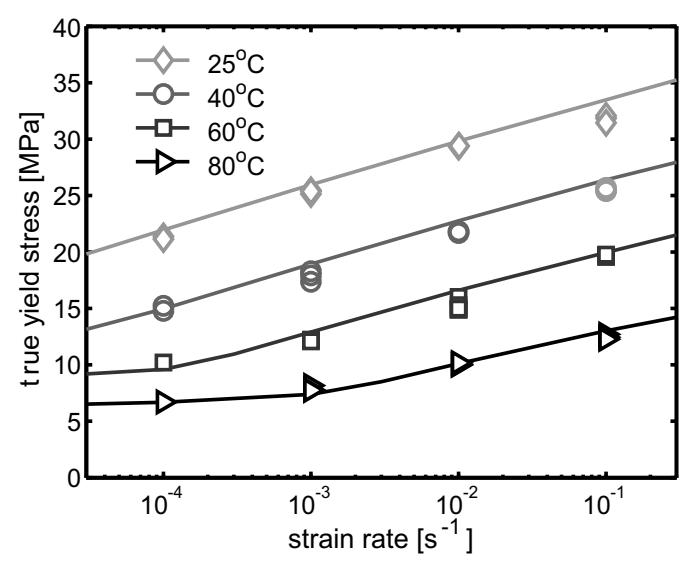

b

Figure 4.8 (a) Compressive true stress-strain curves at varying temperatures and strain rates, predicted by the micromechanical model, and (b) Compressive true yield kinetics of isotropic HDPE. Symbols indicate experimental data.

model. Figure $4.8 \mathrm{~b}$ shows the predicted compressive true yield kinetics at various temperatures, compared to experimental results, which shows a good agreement.

Having predicted the yield kinetics, it is now attempted to predict the long-term failure of isotropic HDPE, dominated by plastic flow (ductile failure). Therefore, the creep behavior of polyethylene under constant force is simulated directly using the multi-scale, micromechanical model, linking the time-to-failure to the applied stress without any additional fitting parameter, often employed in modeling approaches [37, 38]. Figure 4.9 a shows the predicted creep curves of isotropic HDPE under various constant applied stresses at ambient temperature. A comparison of the model simulation with actual experimental creep curves, also shown in this figure, reveals a good agreement. To obtain the time-to-failure from the prediction, a so-called Sherby-Dorn plot [43], i.e. a semi-logarithmic plot of creep rate versus strain, is used, see figure $4.9 \mathrm{~b}$. Failure is assumed to occur at the maximum creep rate in the Sherby-Dorn plot. Finally, in figure 4.10 , the predicted dependence of the time-to-failure on temperature and applied stress is given. Comparison with experimental data shows that both the short-term (figures 4.6 and 4.8) and the long-term (figure 4.10) kinetics of isotropic HDPE are described well by the micromechanical model within the range of temperatures, strain rates and applied loads.

Figure 4.11a illustrates the activity of a selected number of slip systems at the engineering yield stress of $18.15 \mathrm{MPa}$ (maximum in local stress) under a constant applied strain rate of $10^{-4} \mathrm{~s}^{-1}$ in tension at $25^{\circ} \mathrm{C}$. The activity of the same slip systems under a constant applied stress of $18 \mathrm{MPa}$, at the secondary creep stage, where the creep rate remains more or less constant (the minimum in the 


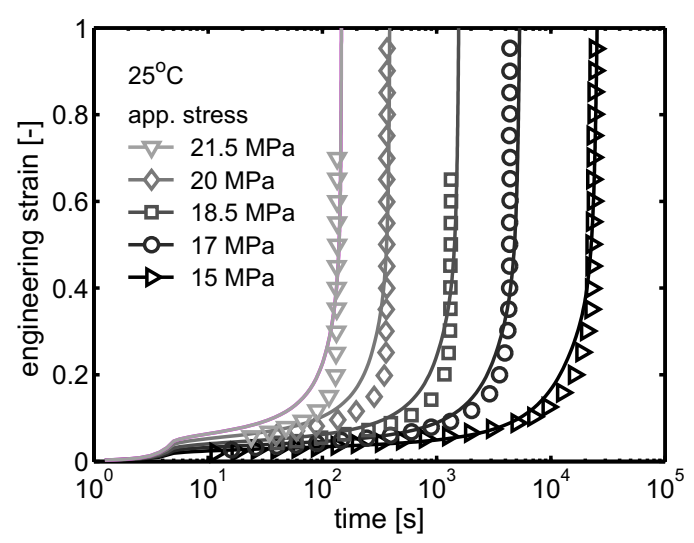

a

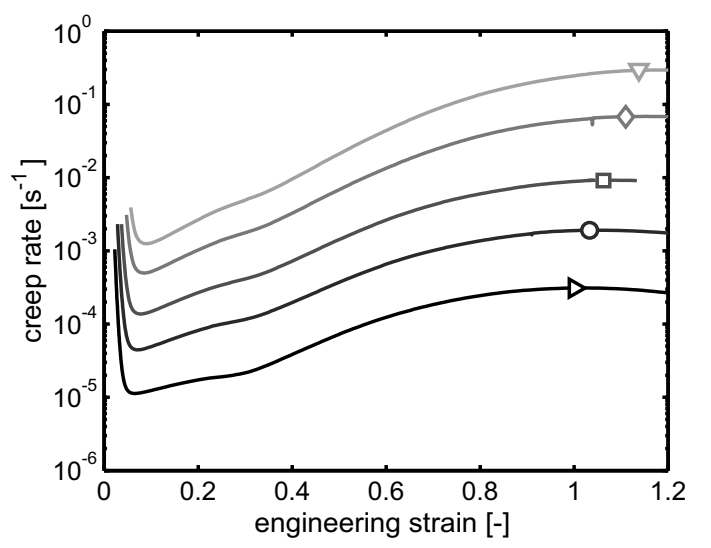

$\mathrm{b}$

Figure 4.9 (a) Predicted creep curves of isotropic HDPE, subjected to different constant loads. Symbols show the experimental creep curves. (b) Sherby-Dorn plots, predicted by micromechanical model. Symbols indicate the maximum creep rate, associated with failure.

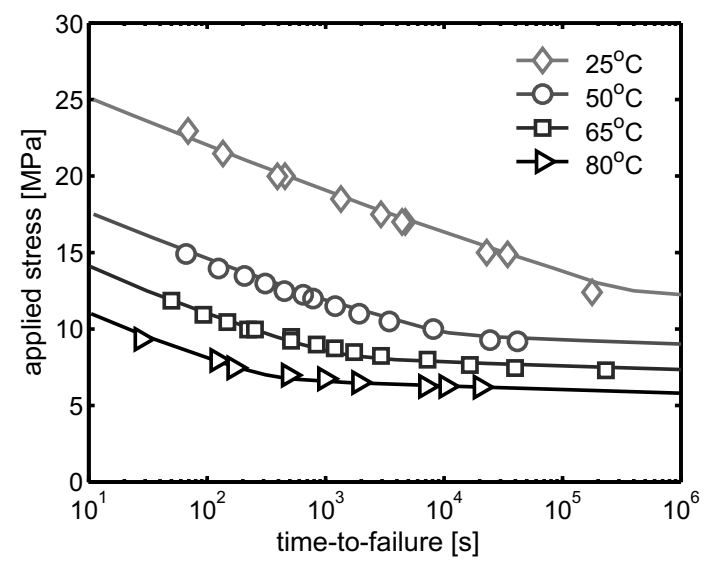

Figure 4.10 Applied stress dependence of time-to-failure at various temperatures. Lines indicate the micromechanical model prediction and symbols give the experimental data.

Sherby-Dorn plot), is shown in figure $4.11 \mathrm{~b}$ for a temperature of $25^{\circ} \mathrm{C}$. In these inverse pole figures, the location of each dot denotes the relative orientation of the loading direction with respect to the crystallographic coordinate system of the corresponding inclusion, and its color shows the magnitude of the resolved shear rate of the indicated slip system. As can be seen, the micromechanical steadystate rates of deformation in static loading are similar to the rates of deformation at the yield point for a constant strain rate. This demonstrates that in polymers, an applied stress results in a steady state rate of plastic flow, and hence failure, 
that are equivalent to the plastic flow at the yield point and failure mode observed under a constant strain rate.

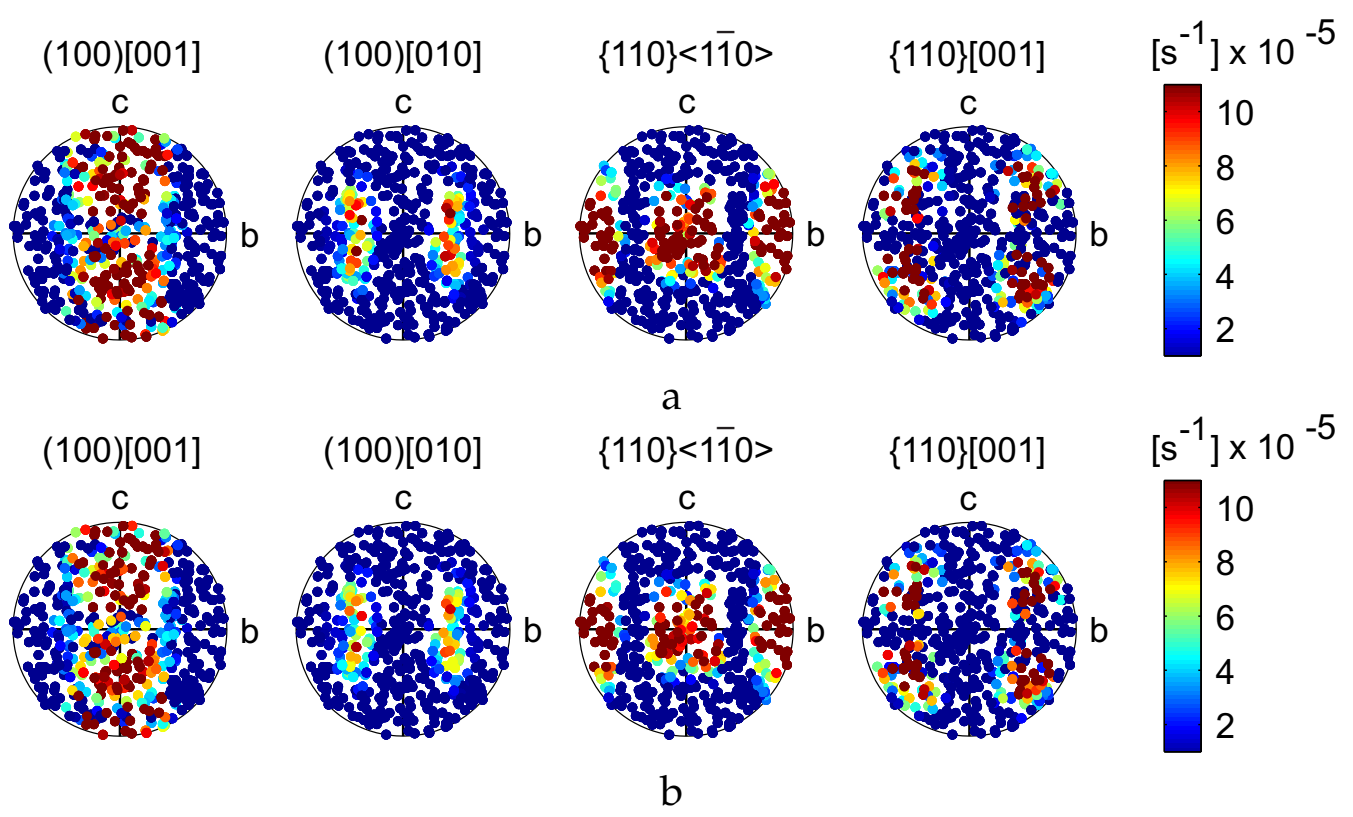

Figure 4.11 Inverse pole figures of the activity of a number of slip systems (a): at the yield stress of $18.15 \mathrm{MPa}$ under a constant applied strain rate of $10^{-4} \mathrm{~s}^{-1}$ in tension at $25^{\circ} \mathrm{C}$, and (b) during the constant steady-state creep rate under a constant applied stress of $18 \mathrm{MPa}$ at $25^{\circ} \mathrm{C}$. Colors represent the magnitude of the resolved shear rate of the specified slip system for each inclusion.

As has already been mentioned, the kinetics of each slip system and the kinetics of the amorphous phase are assumed to include both processes. Here, a physical picture behind that is discussed. Crystallographic slip occurs due to the generation and migration of dislocations within the crystals [27]. The number of mobile dislocations that pre-exist in the crystalline lamellae of semicrystalline polymers is sufficient to initiate the process of plastic deformation [48]. However, during crystallographic slip, dislocations propagate through the crystal and soon emit from the edges of the lamellae, due to the small dimensions of the crystals in semicrystalline polymers. Therefore, new dislocations must be generated and enter the crystals to accommodate the plastic flow. At low rates of deformation, the motion of dislocations through the crystalline domain occurs at a low rate. Then, if temperature is high enough, dislocations can be easily nucleated as a result of thermally-induced translational mobility of chain segments within the crystalline domain, and the resistance of the crystalline lattice to the propagation of dislocations is assumed to be the rate controlling process. However, at 
higher rates of deformation, dislocations emit from the edge of the crystals at a higher rate, and, therefore, a higher rate of nucleation of dislocations is needed for crystallographic slip. Accordingly, more stress is needed to nucleate the amount of dislocations, required to allow for plastic flow. The kinetics of plastic flow, therefore, is controlled by the nucleation of dislocations [79]. Séguéla et al. [30, 31] proposed the mechanism for the nucleation of dislocations to be the migration of stress-induced twist defects along the chain stems. Such defects allow translational mobility of chain segments, also referred to as chain diffusion, and are required for the $\alpha$-relaxation process as well, resulting in relaxation of the interlamellar material in a rate dependent process [32]. Therefore, the kinetics of the relaxation of the amorphous phase is influenced by that of the crystalline phase, and in line with that, the slip kinetics and the amorphous yield kinetics in the model both account for the rate controlling process. The need of having a relaxation process for the amorphous domain in regime I might be due to the intra-inclusion interaction conditions.

\subsection{Conclusions}

The current work is directed towards adding quantitative predictive abilities to a multi-scale micromechanical model for semicrystalline polymers, specifically for their deformation kinetics and long-term failure. The model distinguishes two different scales. At the microscopic level, a two-phase composite entity is employed, comprised of a crystalline lamella that deforms plastically via ratedependent crystallographic slip, and an amorphous domain, with plastic flow being a rate-dependent process. At the macroscopic level, the actual orientation distribution is modeled by an aggregate of these composite inclusions.

A critical issue in achieving the goal of quantitative prediction, is a proper description of the crystallographic slip kinetics and the yield kinetics of the interlamellar material, which are the mechanisms underlying time-dependent, macroscopic failure. Characterization of the kinetics at the microscopic scale has been done using a combined numerical/experimental approach, taking into account uniaxial compression and tension experiments of isotropic HDPE, at various temperatures and strain rates. The kinetics of individual slip systems, together with the kinetics of the amorphous phase, account for the two relaxation mechanisms that are present in the experimentally obtained data for tensile yield kinetics. Comparing the predicted compressive and tensile yield kinetics with experimental data, shows a promising agreement.

Phenomenological studies of failure show that the mode of failure observed in the long-term performance of polymers is similar to that observed in a short- 
term tensile test; necking, associated with decreasing engineering stress. Having predicted that, the creep behavior of isotropic HDPE has also been simulated directly using the micromechanical model, and the time-to-failure under various applied stress and temperatures has been calculated. The predicted stressdependence of the time-to-failure reveals a piece-wise linear relation on a semilogarithmic scale, with the same absolute slope as that of the rate-dependence of the yield kinetics, and provides a good agreement with experimental data.

In the present work, the micromechanical model is characterized based on the response of initially isotropic semicrystalline polymers. To characterize the crystallographic slip kinetics and the amorphous yield kinetics, their properties are related to the engineering level, in which experiments can be performed. However, what is observed at the macroscopic level is the effective contribution of six different types of slip systems, each possibly having different properties, and the yield kinetics of the amorphous material. Therefore, it is not possible to completely distinguish between all slip systems using macroscopic yield kinetics of an isotropic systems, and in line with that the properties of some slip systems are assumed to be the same in the micromechanical model.

The next step will be the evaluation of the model prediction for materials with an initially oriented microstructure. This might require a re-evaluation of the individual slip kinetics. Furthermore, the model does not yet take into account the pronounced dependence on lamellar thickness that is experimentally observed. 


\section{A Appendix: Multi-scale constitutive model}

In this appendix, the material models used for the constituent phases, and the composite inclusion model for semicrystalline polymers are presented.

\section{A.1 Crystalline phase}

The elastic behavior of the crystalline phase is characterized by a fourth order elasticity tensor ${ }^{4} \mathcal{C}^{\mathrm{c}}$ which linearly relates the second Piola-Kirchhoff stress tensor $\tau^{\mathrm{c}}$ and the Green-Lagrange strain tensor $E_{\mathrm{e}}^{\mathrm{c}}$ :

$$
\tau^{\mathrm{C}}={ }^{4} \mathcal{C}^{\mathrm{c}}: E_{\mathrm{e}}^{\mathrm{c}}
$$

with

$$
\boldsymbol{\tau}^{\mathrm{c}}=J_{\mathrm{e}}^{\mathrm{c}} \boldsymbol{F}_{\mathrm{e}}^{\mathrm{c}^{-1}} \cdot \sigma^{\mathrm{c}} \cdot \boldsymbol{F}_{\mathrm{e}}^{\mathrm{c}^{-\mathrm{T}}} \quad \text { and } \quad \boldsymbol{E}_{\mathrm{e}}^{\mathrm{c}}=\frac{1}{2}\left(\boldsymbol{F}_{\mathrm{e}}^{\mathrm{c}^{\mathrm{T}}} \cdot \boldsymbol{F}_{\mathrm{e}}^{\mathrm{c}}-\boldsymbol{I}\right),
$$

with $J_{\mathrm{e}}^{\mathrm{c}}=\operatorname{det}\left(\boldsymbol{F}_{\mathrm{e}}^{\mathrm{c}}\right)$ the volume ratio, $\sigma^{\mathrm{c}}$ the Cauchy stress tensor and $\boldsymbol{I}$ the second order identity tensor. The viscoplastic component of the deformation in the crystalline phase, composed of the contributions of all $N_{s}$ physically distinct slip systems, is given by:

$$
\boldsymbol{L}_{\mathrm{p}}^{\mathrm{c}}=\dot{\boldsymbol{F}}_{\mathrm{p}}^{\mathrm{c}} \cdot \boldsymbol{F}_{\mathrm{p}}^{\mathrm{c}-1}=\sum_{\alpha=1}^{N_{s}} \dot{\gamma}^{\alpha} \boldsymbol{P}_{0}^{\alpha} \quad ; \quad \boldsymbol{P}_{0}^{\alpha}=\vec{s}_{0}^{\alpha} \otimes \vec{n}_{0}^{\alpha},
$$

with $\boldsymbol{P}_{0}^{\alpha}$ the nonsymmetric Schmid tensor, given by the dyadic product of the unit slip direction $\vec{s}_{0}^{\alpha}$ and the unit slip plane normal $\vec{n}_{0}^{\alpha}$ of the $\alpha$ th slip system. The resolved shear rate $\dot{\gamma}^{\alpha}$ of each slip system is assumed to be related to the corresponding resolved shear stress $\tau^{\alpha}$, via a modified Eyring flow relation:

$$
\dot{\gamma}^{\alpha}=\dot{\gamma}_{0}^{\alpha} \exp \left(\frac{-\Delta U^{\alpha}}{R T}\right) \sinh \left(\frac{\tau^{\alpha}}{\tau_{0}^{\mathrm{c}}}\right) \exp \left(\frac{\mu^{\alpha} \sigma_{\mathrm{n}}^{\alpha}}{\tau_{0}^{\mathrm{c}}}\right),
$$

with

$$
\tau^{\alpha}=J \sigma^{\mathrm{c}}: P^{\alpha}=\tau^{\mathrm{c}} \cdot C_{\mathrm{e}}^{\mathrm{c}}: P_{0}^{\alpha}
$$

and

$$
\sigma_{\mathrm{n}}^{\alpha}=J \sigma^{\mathrm{c}}: N^{\alpha}=\tau^{\mathrm{c}}: N_{0}^{\alpha}
$$


where $N_{0}^{\alpha}$ is a tensor defined as $N_{0}^{\alpha}=\vec{n}_{0}^{\alpha} \otimes \vec{n}_{0}^{\alpha}$ and $C_{\mathrm{e}}^{\mathrm{c}}$ denotes the elastic right Cauchy-Green deformation tensor.

\section{A.2 Amorphous phase}

The elastic behavior of the amorphous phase is modeled by a generalized neoHookean relationship:

$$
\sigma_{\mathrm{e}}^{\mathrm{a}}=\frac{G^{\mathrm{a}}}{J_{\mathrm{e}}^{\mathrm{a}}} \tilde{\boldsymbol{B}}_{\mathrm{e}}^{\mathrm{a}^{\mathrm{d}}}+K^{\mathrm{a}}\left(J_{\mathrm{e}}^{\mathrm{a}}-1\right) \boldsymbol{I},
$$

where the superscript "d" denotes the deviatoric part, $\tilde{F}_{\mathrm{e}}^{\mathrm{a}}=J_{\mathrm{e}}^{\mathrm{a}-\frac{1}{3}} \boldsymbol{F}_{\mathrm{e}}^{\mathrm{a}}$ is the isochoric elastic deformation gradient tensor and $\tilde{\boldsymbol{B}}_{\mathrm{e}}^{\mathrm{a}}=\tilde{\boldsymbol{F}}_{\mathrm{e}}^{\mathrm{a}} \cdot \tilde{\boldsymbol{F}}_{\mathrm{e}}^{\mathrm{a}^{\mathrm{T}}}$ is the isochoric elastic left Cauchy-Green deformation tensor. $G^{\mathrm{a}}$ and $J_{\mathrm{e}}^{\mathrm{a}}$ are the shear modulus and bulk modulus, respectively.

A viscoplastic Eyring flow rule is employed to relate the effective shear strain rate $\dot{\gamma}_{\mathrm{p}}^{\mathrm{a}}$ to the effective shear stress of the amorphous phase $\tau^{\mathrm{a}}$, defined as

$$
\tau^{\mathrm{a}}=\sqrt{\frac{1}{2} \sigma_{*}^{\mathrm{a}^{\mathrm{d}}}: \sigma_{*}^{\mathrm{a}^{\mathrm{d}}}} \quad \text { with } \quad \sigma_{*}^{\mathrm{a}}=\boldsymbol{R}_{\mathrm{e}}^{\mathrm{a}^{\mathrm{T}}} \cdot \sigma^{\mathrm{a}} \cdot \boldsymbol{R}_{\mathrm{e}}^{\mathrm{a}}-H^{\mathrm{a}},
$$

with $\boldsymbol{R}_{\mathrm{e}}^{\mathrm{a}}$ the rotation tensor, obtained from the polar decomposition of $\boldsymbol{F}_{\mathrm{e}}^{\mathrm{a}}$ and $H^{\mathrm{a}}$ a back stress tensor, which accounts for orientation-induced hardening and is given by:

$$
\boldsymbol{H}^{\mathrm{a}}=\mu_{\mathrm{R}} \frac{\sqrt{N}}{\lambda_{\mathrm{ch}}} \mathcal{L}^{-1}\left(\frac{\lambda_{\mathrm{ch}}}{\sqrt{N}}\right)\left(\boldsymbol{B}_{\mathrm{p}}^{\mathrm{a}}-\lambda_{\mathrm{ch}}^{2} \boldsymbol{I}\right),
$$

where $\lambda_{\mathrm{ch}}=\sqrt{\frac{1}{3} \operatorname{tr}\left(\boldsymbol{B}_{\mathrm{p}}^{\mathrm{a}}\right)}$ represents the stretch of each chain in the eight-chain network model and $\mathcal{L}^{-1}$ is the inverse of the Langevin function. The plastic rate of deformation $D_{\mathrm{p}}^{\mathrm{a}}$ is then defined as:

$$
D_{\mathrm{p}}^{\mathrm{a}}=\frac{\dot{\gamma}_{\mathrm{p}}^{\mathrm{a}}}{\tau^{\mathrm{a}}} \sigma_{*}^{\mathrm{a}^{\mathrm{d}}},
$$

where the effective shear rate $\dot{\gamma}_{p}^{\text {a }}$, is given by:

$$
\dot{\gamma}_{\mathrm{p}}^{\mathrm{a}}=\dot{\gamma}_{0}^{\mathrm{a}} \exp \left(\frac{-\Delta U^{\mathrm{a}}}{R T}\right) \sinh \left(\frac{\tau^{\mathrm{a}}}{a \tau_{0}^{\mathrm{a}}}\right) \exp \left(\frac{-\mu^{\mathrm{a}} p^{\mathrm{c}}}{b \tau_{0}^{\mathrm{a}}}\right),
$$


with $p^{\mathrm{c}}$ the pressure within the crystalline domain, due to the fact that the kinetics of the relaxation of the amorphous domain originates from that of the crystalline phase, and given by:

$$
p^{\mathrm{c}}=-\frac{1}{3} \sigma^{\mathrm{c}}: I
$$

\section{A.3 Composite inclusion model}

The inclusion-averaged deformation gradient $\boldsymbol{F}^{\mathrm{I}}$ and Cauchy stress $\sigma^{\mathrm{I}}$ of each individual composite are given by:

$$
\begin{gathered}
\boldsymbol{F}^{\mathrm{I}}=f_{0}^{\mathrm{c}} \boldsymbol{F}^{\mathrm{c}}+\left(1-f_{0}^{\mathrm{c}}\right) \boldsymbol{F}^{\mathrm{a}}, \\
\boldsymbol{\sigma}^{\mathrm{I}}=f^{\mathrm{c}} \boldsymbol{\sigma}^{\mathrm{c}}+\left(1-f^{\mathrm{c}}\right) \boldsymbol{\sigma}^{\mathrm{a}} .
\end{gathered}
$$

The two phases are assumed to be kinematically compatible and in equilibrium across the interface. The compatibility and equilibrium conditions on the interface can be written as:

$$
\begin{gathered}
\boldsymbol{F}^{\mathrm{a}} \cdot \vec{x}_{0}^{\mathrm{I}}=\boldsymbol{F}^{\mathrm{c}} \cdot \vec{x}_{0}^{\mathrm{I}}=\boldsymbol{F}^{\mathrm{I}} \cdot \vec{x}_{0}^{\mathrm{I}}, \\
\sigma^{\mathrm{a}} \cdot \vec{n}^{\mathrm{I}}=\sigma^{\mathrm{c}} \cdot \vec{n}^{\mathrm{I}}=\sigma^{\mathrm{I}} \cdot \vec{n}^{\mathrm{I}},
\end{gathered}
$$

where $\vec{n}^{\mathrm{I}}=\vec{e}_{3}^{\mathrm{I}}$ denotes the unit normal vector of the interface, and $\vec{x}_{0}^{\mathrm{I}}$ denotes an arbitrary vector in the plane of interface, spanned by $\vec{e}_{1}^{I}$ and $\vec{e}_{2}^{I}$ such that $\vec{e}_{1}^{I} \times \vec{e}_{2}^{I}=$ $\vec{e}_{3}^{I}$.

\section{A.4 Hybrid interaction law}

In the hybrid interaction model, six auxiliary deformation-like unknowns $\hat{U}$ are introduced. The macroscopic Cauchy stress tensor, the macroscopic right stretch tensor an the macroscopic rotation tensor are denoted by $\bar{\sigma}, \bar{U}$ and $\bar{R}$, respectively. Then, for an aggregate of $N^{\mathrm{I}}$ composite inclusions, the following local-global interaction conditions are used:

$$
\begin{gathered}
\boldsymbol{U}^{\mathrm{I}^{i}} \cdot \vec{n}_{0}^{\mathrm{I}^{i}}=\hat{\boldsymbol{U}} \cdot \vec{n}_{0}^{\mathrm{I}^{i}} ; i=1, \ldots, N^{\mathrm{I}}, \\
\sigma^{\mathrm{I}^{i}} \cdot \vec{x}^{I^{i}}=\bar{\sigma} \cdot \vec{x}^{I^{i}} \quad ; \quad i=1, \ldots, N^{\mathrm{I}},
\end{gathered}
$$




$$
\boldsymbol{R}^{\mathrm{I}^{i}}=\overline{\boldsymbol{R}} \quad ; \quad i=1, \ldots, N^{\mathrm{I}},
$$

in combination with the consistency conditions:

$$
\begin{aligned}
& \overline{\boldsymbol{\sigma}}=\sum_{i=1}^{N^{\mathrm{I}}} f^{\mathrm{I}^{i}} \sigma^{\mathrm{I}^{i}}, \\
& \overline{\boldsymbol{u}}=\left(\frac{\bar{J}}{J_{\Sigma}}\right)^{\frac{1}{3}} \sum_{i=1}^{N^{\mathrm{I}}} f_{0}^{\mathrm{I}^{i}} \boldsymbol{U}^{\mathrm{I}^{i}},
\end{aligned}
$$

with $\bar{J}=\sum_{i=1}^{N^{\mathrm{I}}} f_{0}^{\mathrm{I}^{i}} J^{\mathrm{I}^{i}}$ and $J_{\Sigma}=\operatorname{det}\left(\sum_{i=1}^{N^{\mathrm{I}}} f_{0}^{\mathrm{I}^{i}} \boldsymbol{F}^{\mathrm{I}^{i}}\right)$. 


\section{B Appendix: Viscosity expression}

The thermo-rheologically complex response of a semicrystalline polymer is captured here based on the Ree-Eyring [62] modification of the original Eyring expression, assuming that the total resolved shear stress along a slip plane can be expressed by two independent flow processes, with the stress contributions being additive (see figure 4.12):

$$
\tau^{\mathrm{tot}}=\tau^{\mathrm{I}}+\tau^{\mathrm{II}}
$$

Rewriting equation (4.A.4) in terms of resolved shear stress of an individual slip

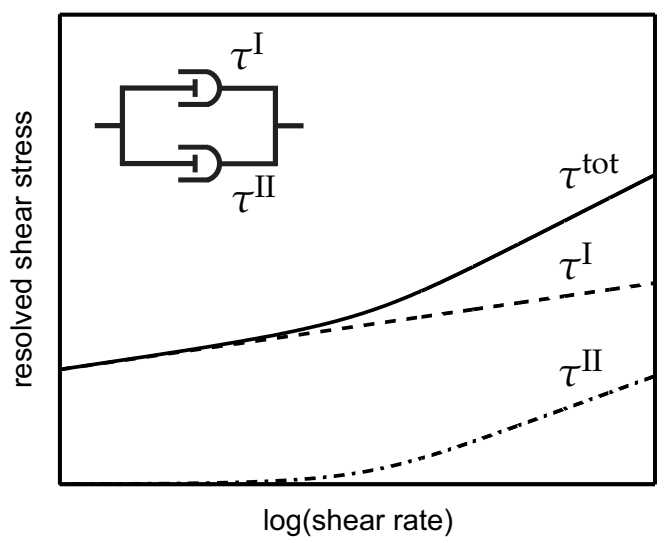

Figure 4.12 Schematic illustration of the modified Eyring rate expression for the slip and amorphous phase kinetics.

plane gives:

$$
\tau^{\pi}=\tau_{0}^{\pi} \sinh ^{-1}\left[\frac{\dot{\gamma}}{\dot{\gamma}_{0}^{\pi}} \exp \left(\frac{\Delta U^{\pi}}{R T}\right) \exp \left(\frac{-\mu \sigma_{\mathrm{n}}}{\tau_{0}^{\mathrm{c}}}\right)\right], \quad \text { with } \quad \pi=\mathrm{I}, \mathrm{II} .
$$

In a similar way, the resolved shear stress in the I+II regime can be expressed as:

$$
\tau^{\mathrm{I}+\mathrm{II}}=\tau_{0}^{\mathrm{I}+\mathrm{II}} \sinh ^{-1}\left[\frac{\dot{\gamma}}{\dot{\gamma}_{0}^{* I+I I}} \exp \left(\frac{-\mu \sigma_{\mathrm{n}}}{\tau_{0}^{\mathrm{c}}}\right)\right],
$$

where $\tau_{0}^{\mathrm{I}+\mathrm{II}}$ represents the rate dependence in the I+II regime, and the temperature dependent pre-exponential constant $\dot{\gamma}_{0}^{*+\mathrm{II}}$ contains a contribution of the two processes. For large stresses, at which $\sinh ^{-1}(x) \approx \ln (2 x)$, equations (4.B.2) 
and (4.B.3) become:

$$
\begin{gathered}
\tau^{\pi}=\tau_{0}^{\pi} \ln [\dot{\gamma}]+\tau_{0}^{\pi} \ln \left[\frac{2}{\dot{\gamma}_{0}^{\pi}} \exp \left(\frac{\Delta U^{\pi}}{R T}\right) \exp \left(\frac{-\mu \sigma_{\mathrm{n}}}{\tau_{0}^{\mathrm{C}}}\right)\right], \quad \text { with } \pi=\mathrm{I}, \mathrm{II} \\
\tau^{\mathrm{I}+\mathrm{II}}=\tau_{0}^{\mathrm{I}+\mathrm{II}} \ln [\dot{\gamma}]+\tau_{0}^{\mathrm{I}+\mathrm{II}} \ln \left[\frac{2}{\dot{\gamma}_{0}^{* \mathrm{I}+\mathrm{II}}} \exp \left(\frac{-\mu \sigma_{\mathrm{n}}}{\tau_{0}^{\mathrm{C}}}\right)\right],
\end{gathered}
$$

respectively, where $\tau_{0}^{\mathrm{I}+\mathrm{II}}$ and $\dot{\gamma}_{0}^{*+\mathrm{II}}$ can be derived from equations (4.B.4) and (4.B.5), while $\tau^{\mathrm{I}+\mathrm{II}}=\tau^{\mathrm{I}}+\tau^{\mathrm{II}}$, as:

$$
\begin{aligned}
& \tau_{0}^{\mathrm{I}+\mathrm{II}}=\tau_{0}^{\mathrm{I}}+\tau_{0}^{\mathrm{II}} \\
& \dot{\gamma}_{0}^{{ }^{\mathrm{I}+\mathrm{II}}}=2 \exp \left(-\frac{\tau_{0}^{\mathrm{I}} \ln \left[\frac{2}{\dot{\gamma}_{0}^{\mathrm{I}}} \exp \left(\frac{\Delta U^{\mathrm{I}}}{R T}\right)\right]+\tau_{0}^{\mathrm{II}} \ln \left[\frac{2}{\dot{\gamma}_{0}^{\mathrm{II}}} \exp \left(\frac{\Delta U^{\mathrm{II}}}{R T}\right)\right]}{\tau_{0}^{\mathrm{I}}+\tau_{0}^{\mathrm{II}}}\right) .
\end{aligned}
$$

The stress and temperature dependence of the solid state viscosity $\eta$ in the I and I+II regimes can be obtained by replacing equations (4.B.2) and (4.B.3) in $\eta=\frac{\tau}{\dot{\gamma}}$ and defining the zero viscosity as $\eta_{0}=\frac{\tau_{0}}{\dot{\gamma}_{0}}$. Figure 4.13 a shows the combination of the viscosity of the I regime with the I+II regime. At low shear stress levels, $\eta^{\mathrm{I}}$ is much larger than $\eta^{\mathrm{I}+\mathrm{II}}$ (note the logarithmic scale), whereas at high shear stress levels, $\eta^{\mathrm{I}+\mathrm{II}}$ is dominant. The viscosity response over the complete range of shear stresses can be approximated by addition of these two viscosity regimes:

$$
\eta^{\text {tot }}=\eta^{\mathrm{I}}+\eta^{\mathrm{I}+\mathrm{II}}=\eta_{0}^{\mathrm{I}}\left[\frac{\frac{\tau}{\tau_{0}^{\mathrm{I}}}}{\sinh \left(\frac{\tau}{\tau_{0}^{\mathrm{I}}}\right)} \exp \left(\frac{\Delta U^{\mathrm{I}}}{R T}\right)+\frac{\eta_{0}^{*^{\mathrm{I}+\mathrm{II}}}}{\eta_{0}^{\mathrm{I}}} \frac{\frac{\tau}{\tau_{0}^{I+I I}}}{\sinh \left(\frac{\tau}{\tau_{0}^{\mathrm{I}+\mathrm{II}}}\right)}\right] \exp \left(\frac{-\mu \sigma_{\mathrm{n}}}{\tau_{0}^{\mathrm{C}}}\right)
$$

Due to the logarithmic dependence of the viscosity to stress, the contribution of $\eta^{\mathrm{I}+\mathrm{II}}$ is negligible in the I regime, and that of $\eta^{\mathrm{I}}$ is negligible in the I+II regime. The resulting shear stress dependence of the shear rate, depicted in figure $4.13 \mathrm{~b}$, is then given by:

$$
\dot{\gamma}=\left[\frac{\dot{\gamma}_{0}^{\mathrm{I}} \exp \left(-\frac{\Delta U^{\mathrm{I}}}{R T}\right) \sinh \left(\frac{\tau}{\tau_{0}^{\mathrm{I}}}\right) \dot{\gamma}_{0}^{* \mathrm{I}+\mathrm{II}} \sinh \left(\frac{\tau}{\tau_{0}^{\mathrm{I}+\mathrm{II}}}\right)}{\dot{\gamma}_{0}^{\mathrm{I}} \exp \left(-\frac{\Delta U^{\mathrm{I}}}{R T}\right) \sinh \left(\frac{\tau}{\tau_{0}^{\mathrm{I}}}\right)+\dot{\gamma}_{0}^{*^{\mathrm{I}+\mathrm{II}}} \sinh \left(\frac{\tau}{\tau_{0}^{I+I I}}\right)}\right] \exp \left(\frac{\mu \sigma_{\mathrm{n}}}{\tau_{0}^{\mathrm{C}}}\right) .
$$




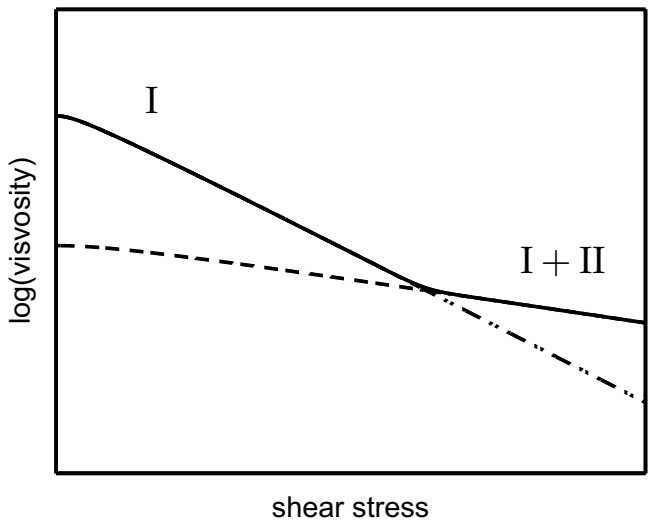

a

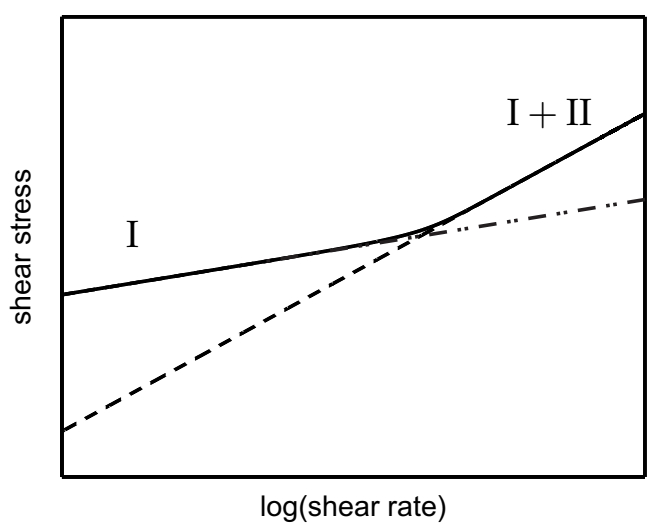

$\mathrm{b}$

Figure 4.13 (a) Description of the I and I+II viscosity regimes as a function of the shear stress. (b) The resulting shear stress as a function of logarithm of resolved shear rate.

\section{References}

[1] F.C. Frank, A. Keller, A. O'Connor, and H.H. Wills. Deformation processes in polyethylene interpreted in terms of crystal plasticity. Philosophical Magazine 25:64-74, 1958.

[2] G. Meinel, N. Riorosoff, and A. Peterlin. Plastic deformation of polyethylene. I. Change of morphology during drawing of polyethylene of high density. Journal of Polymer Science, Part A-2 8:1723-1740, 1970.

[3] G. Meinel and A. Peterlin. Plastic deformation of polyethylene. II. Change of mechanical properties during drawing. Journal of Polymer Science, Part A-2 9:67-83, 1971.

[4] R.J. Young, P.B. Bowden, J.M. Ritchie, and J.G. Rider. Deformation mechanisms in oriented high-density polyethylene. Journal of Materials Science 8:23-36, 1973.

[5] P.B. Bowden and R.J. Young. Deformation mechanisms in crystalline polymers. Journal of Materials Science 9:2034-2051, 1974.

[6] Z. Bartczak, R.E. Cohen, and A.S. Argon. Evolution of the crystalline texture of high density polyethylene during uniaxial compression. Macromolecules 25:4692-4704, 1992.

[7] Z. Bartczak, A.S. Argon, and R.E. Cohen. Deformation mechanisms and plastic resistence in single crystal textured high density polyethylene. Macromolecules 25:5036-5053, 1992.

[8] Z. Bartczak, A.S. Argon, and R.E. Cohen. Texture evolution in large strain simple shear deformation of high density polyethylene. Polymer 35:3427-3441, 1994.

[9] L. Lin and A.S. Argon. Structure and plastic deformation of polyethylene. Journal of Materials Science 29:294-323, 1994.

[10] C. G'Sell and J.J. Jonas. Determination of the plastic behaviour of solid polymers at constant true strain rate. Journal of Materials Science 14:583-591, 1979.

[11] C. G'Sell, S. Boni, and S. Shrivastava. Application of the plane simple shear test for determination of the plastic behaviour of solid polymers at large strains. Journal of Materials Science 18:903-918, 1983. 
[12] E.F. Oleinik. Plasticity of semicrystalline flexible-chain polymers at the microscopic and mesoscopic levels. Polymer Science Series C 45:17-117, 2003.

[13] J.M. Andrews and I.M. Ward. The cold-drawing of high density polyethylene. Journal of Materials Science 5:411-417, 1970.

[14] N. Brown and I.M. Ward. The influence of morphology and molecular weight on ductilebrittle transitions in linear polyethylene. Journal of Materials Science 18:1405-1420, 1983.

[15] D.M. Parks and S. Ahzi. Polycrystalline plastic deformation and texture evolution for crystals lacking five independent slip systems. Journal of the Mechanics and Physics of Solids 38:701-724, 1990.

[16] N.W. Brooks, R.A. Duckett, and I.M. Ward. Investigation into double yield points in polyethylene. Polymer 33:1872-1880, 1992.

[17] A. Galeski, Z. Bartczak, A.S. Argon, and R.E. Cohen. Morphological alterations during texture-producing plastic plane strain compression of high-density polyethylene. Macromolecules 25:5705-5718, 1992.

[18] B.J. Lee, D.M. Parks, and S. Ahzi. Micromechanical modeling of large plastic deformation and texture evolution in semicrystalline polymers. Journal of the Mechanics and Physics of Solids 41:1651-1687, 1993.

[19] B.J. Lee, A.S. Argon, D.M. Parks, S. Ahzi, and Z. Bartczak. Simulation of large strain plastic deformation and texture evolution in high density polyethylene. Polymer 34:3555-3575, 1993.

[20] S. Ahzi, B.J. Lee, and R.J. Asaro. Plasticity and anisotropy evolution in crystalline polymers. Materials Science and Engineering: A 189:35-44, 1994.

[21] N.W.J. Brooks, R.A. Duckett, and I.M. Ward. Modeling of double yield points in polyethylene: temperature and strain-rate dependence. Journal of Rheology 39:425-436, 1995.

[22] N.W.J. Brooks, R.A. Duckett, and I.M. Ward. Temperature and strain-rate dependence of yield stress of polyethylene. Journal of Polymer Science Part B: Polymer Physics 36:2177-2189, 1998.

[23] J.A.W. van Dommelen, D.M. Parks, M.C. Boyce, W.A.M Brekelmans, and F.P.T Baaijens. Micromechanical modeling of the elasto-viscoplastic behavior of semi-crystalline polymers. Journal of the Mechanics and Physics of Solids 51:519-541, 2003.

[24] J.A.W. van Dommelen, B.A.G. Schrauwen, L.C.A. van Breemen, and L.E. Govaert. Micromechanical modeling of the tensile behavior of oriented polyethylene. Journal of Polymer Science, Part B: Polymer Physics 42:2983-2994, 2004.

[25] A. Sedighiamiri, T.B. van Erp, G.W.M. Peters, L.E. Govaert, and J.A.W. van Dommelen. Micromechanical modeling of the elastic properties of semicrystalline polymers: a threephase approach. Journal of Polymer Science, Part B: Polymer Physics 48:2173-2184, 2010.

[26] A. Sedighiamiri, L.E. Govaert, and J.A.W. van Dommelen. Micromechanical modeling of the deformation kinetics of semicrystalline polymers. Journal of Polymer Science, Part B: Polymer Physics 49:1297-1310, 2011.

[27] R.J. Young. A dislocation model for yield in polyethylene. Philosophical Magazine 30, 1974.

[28] R.J. Young. Screw dislocation model for yield in polyethylene. Materials forum 11:210-216, 1988. 
[29] L.G. Shadrake and F. Guiu. Dislocations in polyethylene crystals: line energies and deformation modes. Philosophical Magazine 34:565-581, 1976.

[30] R. Séguéla, V. Gaucher-Miri, and S. Elkoun. Plastic deformation of polyethylene and ethylene copolymers: Part I. Homogeneous crystal slip and molecular mobility. Journal of Material Science 33:1273-1279, 1998.

[31] R. Séguéla. Dislocation approach to the plastic deformation of semicrystalline polymers: kinetic aspects for polyethylene and polypropylene. Journal of Polymer Science, Part B: Polymer Physics 40:593-601, 2002.

[32] R.H. Boyd. Relaxation processes in crystalline polymers: molecular interpretation - a review. Polymer 26:1123-1133, 1985.

[33] S. Nikolov and D. Raabe. Yielding of polyethylene through propagation of chain twist defects: temperature, stem length and strain-rate dependence. Polymer 47:1696-1703, 2006.

[34] C. G'Sell, S. Boni, and S. Shrivastava. Application of the plane simple shear test for determination of the plastic behaviour of solid polymers at large strains. Journal of Materials Science 18:903-918, 1983.

[35] T Ree and H. Eyring. Theory of non-Newtonian flow. I. Solid plastic system. Journal of Applied Physics 26:793-800, 1955.

[36] E.T.J. Klompen, T.A.P. Engels, L.C.A. van Breemen, P.J.G. Schreurs, L.E. Govaert, and H.E.H. Meijer. Quantitative Prediction of Long-Term Failure of Polycarbonate. Macromolecules 38:7009-7017, 2005.

[37] T.B. van Erp, C.T. Reynolds, T. Peijs, J.A.W. van Dommelen, and L.E. Govaert. Prediction of yield and long-term failure of oriented polypropylene: kinetics and anisotropy. Journal of Polymer Science Part B: Polymer Physics 47:2026-2035, 2009.

[38] T.A.P. Engels, S.H.M. Söntjens, T.H. Smit, and L.E. Govaert. Time-dependent failure of amorphous polylactides in static loading conditions. Journal of Materials Science: Materials in Medicine 21:89-97, 2010.

[39] M.D. Mindel and N. Brown. Creep and recovery of polycarbonate. Journal of Materials Science 8:863-870, 1973.

[40] J.M. Crissman and L.J. Zapas. Creep failure and fracture of polyethylene in uniaxial extension. Polymer Engineering and Science 19:99-103, 1979.

[41] U.W. Gedde, J. Viebke, H. Leijstrom, and M. Ifwarson. Long-term properties of hot-water polyolefin pipes - a review. Polymer Engineering and Science 34:1773-1787, 1994.

[42] R.W. Lang, A. Stern, and G. Doerner. Applicability and limitations of current lifetime prediction models for thermoplastics pipes under internal pressure. Die Angewandte Makromolekulare Chemie 247:131-145, 1997.

[43] O.D. Sherby and J.E. Dorn. Anelastic creep of polymethyl methacrylate. Journal of the Mechanics and Physics of Solids 6:145-162,1958.

[44] E.H. Lee. Elastic-plastic deformation at finite strains. Journal of Applied Mechanics: Transactions of the ASME 36:1-6, 1969.

[45] A. Galeski. Strength and toughness of crystalline polymer systems. Progress in Polymer Science 28:1643-1699, 2003. 
[46] B. Crist, C.J. Fisher, and P.R. Howard. Mechanical properties of model polyethylenes: tensile elastic modulus and yield stress. Macromolecules 22:1709-1718, 1989.

[47] R.H. Boyd. Relaxation processes in crystalline polymers: experimental behaviour - a review. Polymer 26:323-347, 1985.

[48] Z. Bartczak and A. Galeski. Plasticity of semicrystalline polymers. Macromolecular Symposia 294:67-90, 2010.

[49] K. Schmidt-Rohr and H.W. Spiess. Chain diffusion between crystalline and amorphous regions in polyethylene detected by 2D exchange carbon-13 NMR. Macromolecules 24:5288$5293,1991$.

[50] M.C. Boyce, D.M. Parks, and A.S. Argon. Large inelastic deformation of glassy polymers. part I: rate dependent constitutive model. Mechanics of Materials 7:15-33, 1988.

[51] E.M. Arruda and M.C. Boyce. A three-dimensional constitutive model for the large stretch behavior of rubber elastic materials. Journal of the Mechanics and Physics of Solids 41:389-412, 1993.

[52] P.I. Vincent. The necking and cold-drawing of rigid plastics. Polymer 1:7-19, 1960.

[53] R.W. Truss, P.L. Clarke, R.A. Duckett, and I.M. Ward. The dependence of yield behavior on temperature, pressure, and strain rate for linear polyethylenes of different molecular weight and morphology. Journal of Polymer Science: Polymer Physics Edition 22:191-209, 1984.

[54] Y. Liu and R.W. Truss. A study of tensile yielding of isotactic polypropylene. Journal of Polymer Science Part B: Polymer Physics 32:2037-2047, 1994.

[55] D.C. Basset and A.M. Hodge. On the morphology of melt-crystallized polyethylene I. Lamellar profiles. Proceedings of the Royal Society of London A 377:25-37, 1981.

[56] S. Gautam, S. Balijepalli, and G.C. Rutledge. Molecular simulations of the interlamellar phase in polymers: effect of chain tilt. Macromolecules 33:9136-9145, 2000.

[57] R. Hiss, S. Hobeika, C. Lynn, and G. Strobl. Network stretching, slip processes and fragmentation of crystallites during uniaxial drawing of polyethylene and related copolymers. A comparative study. Macromolecules 32:4390-4403, 1999.

[58] R.C. Scogna and R.A. Register. Rate-dependence of yielding in ethylene-methacrylic acid copolymers. Polymer 49:992-998, 2008.

[59] J.A. Roetling. Yield stress behaviour of isotactic polypropylene. Polymer 7:303-306, 1966.

[60] J.A. Roetling. Yield stress behaviour of polymethylmethacrylate. Polymer 6:311-317, 1965.

[61] C. Bauwens-Crowet and J.C. Bauwens. Effect of thermal history on the tensile yield stress of polycarbonate in the $\beta$ transition range. Polymer 24:921-924, 1983.

[62] T. Ree and H. Eyring. Theory of Non-Newtonian Flow. I. Solid Plastic System. Journal of Applied Physics 26:793-800, 1955.

[63] F. Bedoui, J. Diani, G. Regnier, and W. Seiler. Micromechanical modeling of isotropic elastic behavior of semicrystalline polymers. Acta Materialia 54:1513-1523, 2006.

[64] K. Tashiro, M. Kobayashi, and H. Tadakoro. Calculation of the three-dimensional elastic constants of polymer crystals. 2. Application to orthorhombic polyethylene and poly(vinyl alcohol). Macromolecules 11:914-918, 1978.

[65] W.J. Welsh. Thermal-Oxidative satability and degradation of polymers - Physical Properties of Polymers Handbook. AIP Press, 1996. 
[66] R.K. Eby. Thermal generation of vacancies and substitutional sites in crystalline polymers. Journal of Applied Physics 33:2253-2256, 1962.

[67] H.G.H. van Melick, L.E. Govaert, and H.E.H. Meijer. On the origin of Strain hardening in glassy polymers. Polymer 44:2493-2502, 2003.

[68] L.E. Govaert and T.A. Tervoort. Strain hardening of polycarbonate in the glassy state: Influence of temperature and molecular weight. Journal of Polymer Science Part B: Polymer Physics 42:2041-2049, 2004.

[69] L.E. Govaert, T.A.P. Engels, M. Wendlandt, T.A. Tervoort, and U.W. Suter. Does the strain hardening modulus of glassy polymers scale with the flow stress? Journal of Polymer Science Part B: Polymer Physics 46:2475-2481, 2008.

[70] M. Wendlandt, T.A. Tervoort, and U.W. Suter. Strain-hardening modulus of cross-linked glassy poly(methyl methacrylate). Journal of Polymer Science Part B: Polymer Physics 48:14641472, 2008.

[71] J.A. Sauer. Deformation, yield and fracture of polymers at high pressure. Polymer Engineering \& Science 17:150-164, 1977.

[72] W.A. Spitzig and O. Richmond. Effect of hydrostatic pressure on the deformation behavior of polyethylene and polycarbonate in tension and in compression. Polymer Engineering $\mathcal{E}$ Science 19:1129-1139, 1979.

[73] D.J.A. Senden, J.A.W. van Dommelen, and L.E. Govaert. Strain Hardening and its Relation to Bauschinger Effects in Oriented Polymers. Journal of Polymer Science Part B: Polymer Physics 48:1483-1494, 2010.

[74] D.R. Mears, K.D. Pae, and J.A. Sauer. Effects of Hydrostatic Pressure on the Mechanical Behavior of Polyethylene and Polypropylene. Journal of Applied Physics 40:4229-4237, 1969.

[75] M.F. Butler, A.M. Donald, and A.J. Ryan. Time resolved simultaneous small- and wideangle X-ray scattering during polyethylene deformation: 1 . Cold drawing of ethylene- $\alpha-$ olefin copolymers. Polymer 38:5521-5538, 1997.

[76] M.F. Butler, A.M. Donald, and A.J. Ryan. Time resolved simultaneous small- and wide-angle X-ray scattering during polyethylene deformation - II. Cold drawing of linear polyethylene. Polymer 39:39-52, 1998.

[77] V. Gaucher-Miri and R. Séguéla. Tensile yield of polyethylene and related copolymers: mechanical and structural evidences of two thermally activated processes. Macromolecules 30:1158-1167, 1997.

[78] R.P.M. Schrauwen, B.A.G.and Janssen, L.E. Govaert, and H.E.H. Meijer. Intrinsic deformation behavior of semicrystalline polymers. Macromolecules 37:6069-6078, 2004.

[79] A.S. Argon, A. Galeski, and T. Kazmierczak. Rate mechanisms of plasticity in semicrystalline polyethylene. Polymer 46:11798-11805, 2005. 



\title{
Deformation kinetics of oriented polymers 1
}

Chapter 5

\begin{abstract}
The mechanical response of extruded semicrystalline materials, in which a stacked lamellar morphology is commonly observed, depends on the direction of loading with respect to the direction of flow. Plastic deformation and failure are, therefore, both anisotropic. The predictive ability of the micromechanical model, including the characterization of the kinetics of the crystallographic slip and amorphous yield, is evaluated here for oriented high-density polyethylene tapes with different draw ratios. The initial morphology of the material is generated based on pole figures from wide-angle $X$-ray diffraction experiments, which show a strong alignment of molecular chains with the drawing direction for specimens produced with a large draw ratio. The angle between chain direction and lamellar normal direction is found to have a strong influence on the yield kinetics, especially when loading is in the direction of the chain. Anisotropic crystal plasticity alone proves not able to quantitatively describe the macroscopic mechanical response in the solid state hot drawn samples. Most likely, during this process also the amorphous domains became oriented. Therefore, the influence of a loading angle dependent yield kinetics for the amorphous phase is evaluated, and indeed the predictions improve considerably. Finally, the possibilities for characterizing the properties of different crystallographic slip systems are investigated.
\end{abstract}

\footnotetext{
${ }^{1}$ A. Sedighiamiri, L.E. Govaert, J.A.W. van Dommelen, A micromechanical study on the deformation and failure kinetics of oriented semicrystalline polymers, in preparation.
} 


\subsection{Introduction}

The plastic deformation of semicrystalline polymers is complicated due to their hierarchical structure. Various competing phenomena and mechanisms are likely to occur simultaneously, and many morphological and structural factors play a role at different levels [1-5]. Therefore, their mechanical performance, as characterized by elasto-viscoplastic deformation, strongly depends on the morphological features (crystallinity, lamellar thickness and molecular weight), the initial underlying microstructure, as well as the evolution of the microstructure during deformation [6-8]. However, there is yet to exist a quantitative model which can accurately describe the deformation and different failure modes, and explain the deformation kinetics in terms of structural and morphological characteristics.

Young [9, 10] made a first attempt in establishing a direct relation between the crystalline structure and the yield stress of semicrystalline PE. His approach regards that yield involves the activation of [001] screw dislocations, accounting for a single crystallographic slip system, and enables a direct relation between macroscopic yield stress and lamellar thickness. The energy required for the nucleation of screw dislocations from the edge of the crystals, with a Burgers vector parallel to the chain direction, was proposed to be supplied by thermal fluctuations of chain segments within crystals [11]. Séguéla et al. [12, 13] later supported Young's plasticity approach, suggesting that crystallographic slip occurs due to nucleation of screw dislocations from the lateral surface of the crystalline lamellae and proposed an underlying mechanism that relies on chain twist defects that migrate along the chain stems.

In recent years, micromechanically-based models have been increasingly used to understand the plastic deformation in semicrystalline polymers, taking into account the contribution of multiple slip systems, the amorphous phase and the crystallographic and morphological texture evolutions during deformation [1421]. Lee et al. [15, 16] developed a specific micromechanical model in which a rigid-viscoplastic two-phase composite inclusion, consisting of mechanically coupled amorphous and crystalline phases, was used to predict the stressstrain behavior and crystallographic texture evolution of HDPE. This approach was further extended by Van Dommelen et al. [18, 19] to an elasto-viscoplastic framework for large deformation of semicrystalline polymers. In this model, the amorphous phase was assumed to be isotropic elastic with a rate-dependent plastic flow and with strain hardening resulting from molecular orientation. The crystalline phase was modeled as anisotropic elastic with plastic flow occurring via crystallographic slip. Sedighiamiri et al. [21] used a hybrid numerical/experimental procedure to add quantitative predictive abilities to 
this model by characterization of the stress-dependence of the rate of plastic deformation, the slip kinetics, for isotropic semicrystalline polyethylene.

However, semicrystalline polymeric products are usually manufactured and shaped in their molten state, using processing operations as injection molding or film and sheet extrusion, during which shear and elongational flows induce orientation prior to crystallization (flow-induced orientation). Orientationdependent mechanical properties in semicrystalline polymers could also be due to plastic deformation during drawing or rolling processes (deformation-induced orientation) [22-24]. The resulting morphology is quite different from what is found for isotropic polymers. The stacked lamellar morphology, commonly observed in extruded semicrystalline materials [25, 26], gives rise to a strong influence of the extrusion direction with respect to the loading direction on the deformation kinetics, causing plastic deformation and failure to be anisotropic [27, 28]. This was confirmed by Van Dommelen et al. [20], who performed tensile tests on melt-extruded HDPE samples at different angles with respect to the extrusion direction. Their results illustrate that when loaded in extrusion direction, a homogeneous deformation was observed throughout the entire sample, whereas a neck was formed when the material was loaded perpendicular to the extrusion direction. The composite inclusion model was used to describe these effects in a qualitative sense.

In the present work, the relation between the initially oriented microstructure and the deformation kinetics of oriented semicrystalline polymers is investigated using a multi-scale micromechanical model [18, 21]. The mechanical behavior at the macroscopic scale is modeled by an aggregate of layered two-phase composite inclusions. The initial orientation distribution is generated based on pole figures of orientation distributions obtained from wide angle X-ray scattering experiments. Oriented high density polyethylene tapes are considered as case studies. The kinetics of macroscopic plastic flow strongly depends on the rate-dependence of slip along crystallographic planes, together with the yield kinetics of the amorphous domain. Therefore, a key issue is a proper description of the stress-dependence of the microscopic rate of plastic deformation for both crystalline and amorphous domains. It is found that due to the presence of extended chains in the oriented amorphous domains in the drawn samples, the orientation-dependent macroscopic plastic flow is predominantly governed by the yield kinetics of the amorphous phase. Therefore, the necessity of modeling an anisotropic amorphous domain for an accurate quantitative prediction is discussed. Finally, the possibilities for identifying the properties of different crystallographic slip systems are explored. 


\subsection{Experimental}

\subsubsection{Material}

The material used is a high density polyethylene, supplied by DSM (Stamylan HD 9089S), with $M_{\mathrm{w}}=70,000[\mathrm{~g} / \mathrm{mol}]$ and $M_{\mathrm{n}}=11,000$ [g/mol]. HDPE tapes were monoextruded using a Collin E20-T single screw extruder fitted with a 100 $\mathrm{mm}$ coat hanger die. The extrudate was quenched on Collin CR-72 rolls at a temperature of $15^{\circ} \mathrm{C}$ and then collected on a spool. Solid state drawing was performed at a temperature of $120^{\circ} \mathrm{C}$, and different tapes with draw ratios $\lambda$ of 1 , 4 and 6 were made.

\subsubsection{Crystallinity}

Wide angle X-ray scattering (WAXS) experiments were performed on the tapes and the weight fraction of crystals of the oriented samples was determined to be $60 \%, 66 \%$ and $63 \%$ for tapes with draw ratios of 1,4 and 6 , respectively. Figure 5.1 gives the recorded WAXS pattern of HDPE tapes with different draw ratios. The

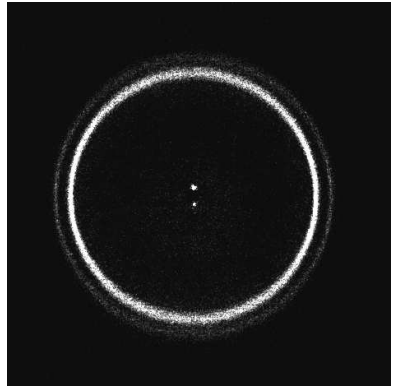

a

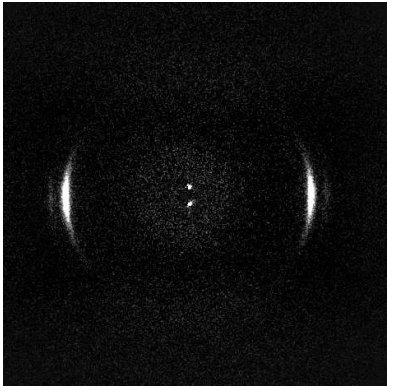

b

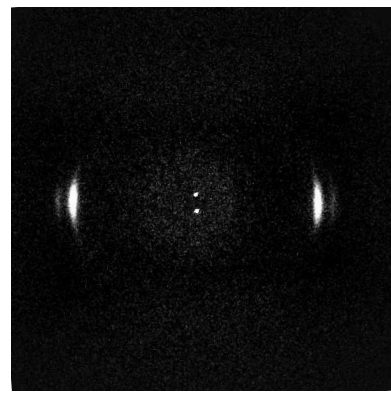

C

Figure 5.1 WAXS patterns of HDPE tapes with a draw ratio of $\lambda=1(\mathrm{a}), \lambda=4$ (b) and $\lambda=6$ (c).

tape with a draw ratio of $\lambda=1$ is found to be isotropic, as its WAXS pattern shows full Debye rings of the reflection of the crystallographic planes. Apparently, flow induced crystallization did not occur during the extrusion process. From the weight fraction of crystals of this sample, the volumetric degree of crystallinity is obtained to be approximately $57 \%$, assuming $\rho_{c}=1000 \mathrm{~kg} / \mathrm{m}^{3}$, for the crystal density and $\rho_{a}=855 \mathrm{~kg} / \mathrm{m}^{3}$, for the isotropic amorphous density [29]. WAXS patterns of the other two samples (figures 5.1b and 5.1k) indicate a rather strong orientation. Bartczak et al. [30] estimated the density of the amorphous 
component in uniaxially oriented HDPE samples. A density of about $\rho_{a}=$ $960 \mathrm{~kg} / \mathrm{m}^{3}$ was observed for a HDPE, compressed to a compression ratio of 6.4, which is very close to the density of the crystals, suggesting a very high degree of ordering of the molecular chains in the amorphous fraction. Due to lack of information about the density of the oriented amorphous fraction, which makes the estimation of the volumetric degree of crystallinity uncertain, the volumetric degree of crystallinity of all samples is set to be $60 \%$ in this study. A concise discussion on the effect of crystallinity on the deformation kinetics will be given in section 5.3.4.

\subsubsection{Orientation measurement}

The oriented HDPE tapes were analyzed by additional wide angle X-ray scattering experiments, and pole figures were constructed to characterize the three dimensional crystallographic orientation distribution. To do that, samples were positioned on a goniometer with the rotation axis in the middle of the ND-TD plane, with ND denoting the thickness direction and TD showing the width direction. The samples were then rotated around the machine direction (MD), collecting WAXS patterns, to create full pole figures of the crystallographic orientation distribution. Figure 5.2 shows a schematic illustration of the setup for orientation measurement.

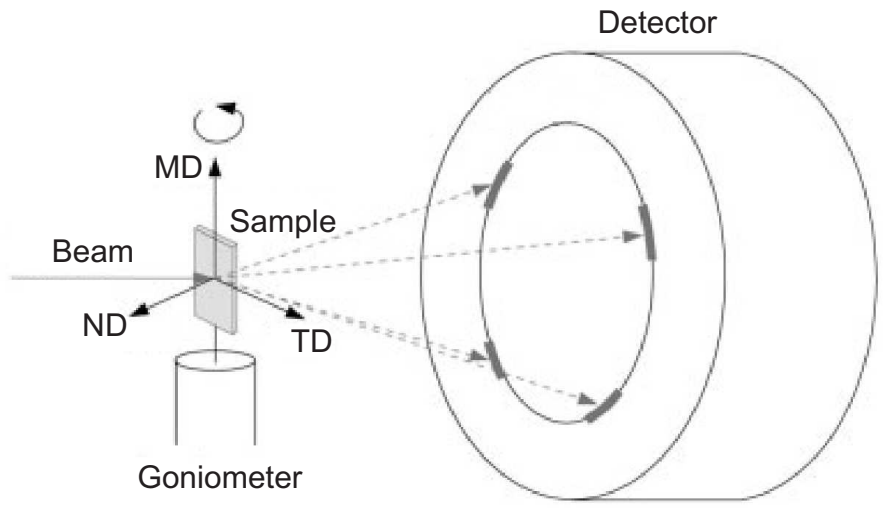

Figure 5.2 Schematic illustration of the setup for orientation distribution measurement.

The resulting pole figures of orientation distributions of two crystallographic planes are shown in figure 5.3 for samples with draw ratios of 4 and 6 . It is observed that the (200) plane normals, corresponding to the crystallographic $a$-axes, and (110) plane normals are oriented in the ND-TD plane, being perpendicular to the draw direction, with an intensity maximum in the center. 
HDPE possesses an orthorhombic unit cell, i.e. the crystallographic directions are orthogonal. Therefore, the crystallographic $b$-axes, corresponding to the lamellar growth directions, should also be oriented perpendicular to the draw direction, and the crystallographic $c$-axes, denoting the molecular chain axes, are oriented preferentially in the draw direction, corresponding to a stacked lamellar morphology.
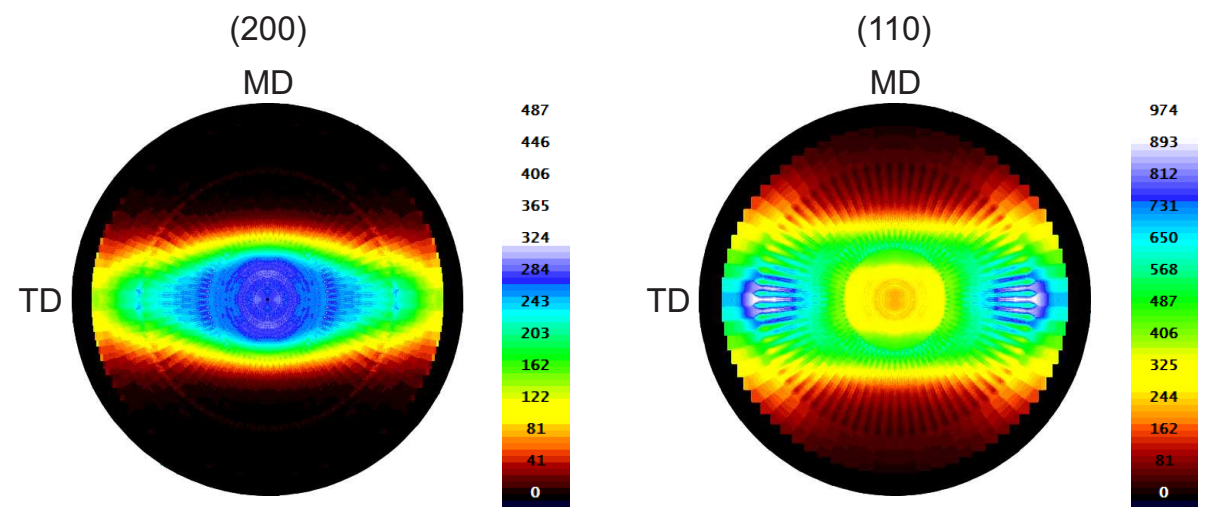

a
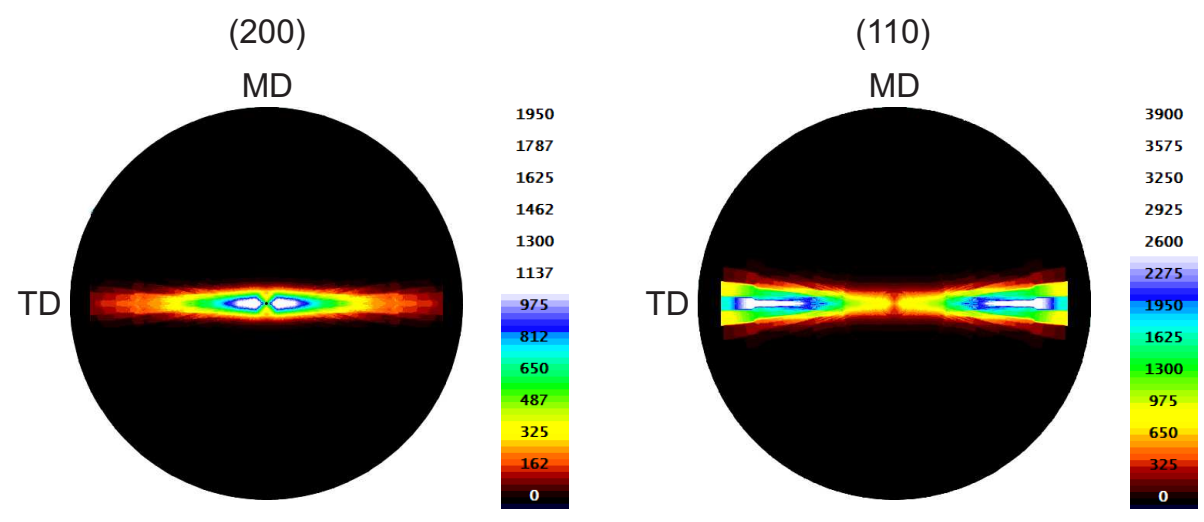

b

Figure 5.3 Pole figures of orientation distribution for HDPE tape with draw ratios of 4 (a) and $6(b)$.

For uniaxially oriented systems, a quantitative measure of orientation can be 
given by the Hermans' orientation factor $\left(f_{H}\right)$, defined as [31]:

$$
f_{H}=\frac{3\left\langle\cos ^{2} \phi\right\rangle-1}{2}
$$

with $\phi$ the angle between the unit whose orientation state is of interest (e.g. crystallographic axes) and a reference axis (e.g. fiber or machine direction). $f_{H}=1$ indicates perfect orientation, $f_{H}=0$ indicates random orientation, and $f_{H}=-0.5$ indicates an orthogonal orientation. Table 5.1 lists the Hermans' orientation distribution factors obtained for the principal crystallographic axes.

Table 5.1 Hermans' orientation distribution factors for crystallographic directions.

\begin{tabular}{ccccc}
\hline sample & draw ratio & $f_{a}$ & $f_{b}$ & $f_{c}$ \\
\hline 1 & 4 & -0.40 & -0.29 & 0.70 \\
2 & 6 & -0.45 & -0.48 & 0.94 \\
\hline
\end{tabular}

\subsubsection{Mechanical testing}

For uniaxial tensile tests, dogbone-shaped samples were cut from the tapes with different angles $\left(0^{\circ}, 20^{\circ}\right.$ and $\left.50^{\circ}\right)$ with respect to the machine direction (drawing direction), as shown schematically in figure 5.4. Tensile tests were performed

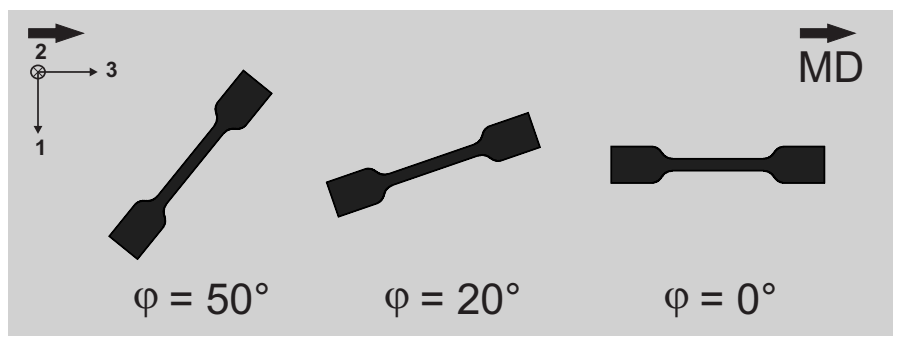

Figure 5.4 Schematic illustration of orientation of samples cut from the tapes with different angles with respect to the machine direction.

at constant, linear strain rates varying from $10^{-4} \mathrm{~s}^{-1}$ up to $10^{-2} \mathrm{~s}^{-1}$ at room temperature. 


\subsection{Model description}

The constitutive behavior of semicrystalline material is modeled by an aggregate of a discrete number of layered two-phase composite inclusions [15, 16, 18]. Each composite inclusion is comprised of a crystalline lamella and an amorphous layer. These two phases are mechanically coupled by enforcing kinematical compatibility and traction equilibrium on their interface. A microstructural elasto-viscoplastic constitutive model is used for both constituent phases. In this section, the material models for the elastic and viscoplastic behavior of each phase together with the aggregate model are briefly summarized. A more detailed discussion of the models is given in chapters 3 and 4 .

\subsubsection{Crystalline phase}

The crystalline phase consists of regularly ordered molecular chains, causing highly anisotropic elastic properties, with a high modulus in the chain direction, and a plastic deformation that is primarily governed by crystallographic slip up to moderate strains [2, 32-34]. Polymer crystals may also deform plastically by mechanical twinning and stress-induced martensitic phase transformation [2, 8, 34], but these two mechanisms are left out of consideration, since crystallographic slip is the predominant deformation mode and can accommodate larger plastic deformations than the other two mechanisms.

The elastic component of the deformation in the crystalline domain is characterized by an anisotropic fourth order elasticity tensor, which linearly relates the Green-Lagrange strain tensor to the elastic second Piola-Kirchhoff stress measure.

The viscoplastic flow behavior of the crystalline phase is described by a ratedependent crystal plasticity model. In this model, the plastic flow rate of the crystalline lamella, consisting of a single crystal, is composed of the contributions of all $N_{s}$ physically distinct slip systems, being 8 for high density polyethylene. The shear rate of each slip system is assumed to be related to the corresponding resolved shear stress via a viscoplastic Eyring flow rule [35]:

$$
\dot{\gamma}=\dot{\gamma}_{0}^{*}(T) \sinh \left(\frac{\tau}{\tau_{0}^{\mathrm{c}}}\right),
$$

where

$$
\dot{\gamma}_{0}^{*}(T)=\dot{\gamma}_{0} \exp \left(\frac{-\Delta U}{R T}\right)
$$


captures the temperature dependence of the slip kinetics, with $\Delta U$ the activation energy of the slip system, $R$ the universal gas constant and $T$ the absolute temperature.

\subsubsection{Amorphous phase}

The amorphous phase consists of an assembly of randomly coiled macromolecules, strongly connected to their adjacent crystalline lamellae through many chains crossing the crystalline/amorphous interface, such as tight or loose chain ends, cilia, and intercrystalline links [36]. In the case of polyethylene, the glass transition temperature of the amorphous phase is below room temperature, causing the amorphous phase to be in the rubbery state. However, there are some indications in literature [37, 38] that suggest that the interlamellar layers in a semicrystalline polymeric materials may be stiffer than purely bulk amorphous materials due to the confinement of amorphous layers between thick crystalline lamellae.

Furthermore, due to random thermal fluctuations, chain segments in the crystalline phase can migrate into the amorphous domain. Similarly, chain ends or loose chain folds can escape from the amorphous phase into the crystalline lamellae. This process of chain diffusion [39], generally referred to as $\alpha$-relaxation, results in a redistribution of chain segments that constitute the interlamellar layer. Therefore, the relaxation of the interlamellar material also occurs in a ratedependent process at a rate controlled by translational mobility of chain segments within the crystals [40]. In line with that, also the amorphous phase is modeled as elasto-viscoplastic.

The elastic deformation of the amorphous phase is modeled by a generalized neo-Hookean relationship, characterized by shear and bulk moduli. An Eyring flow relation is used to relate the effective shear strain rate and the effective shear stress:

$$
\dot{\gamma}=\dot{\gamma}_{0}^{*}(T) \sinh \left(\frac{\tau}{a \tau_{0}^{\mathrm{a}}}\right)
$$

with $a=1.15$ a constant accounting for the difference in the definition of the effective shear stress of the amorphous phase and the resolved shear stress of the crystalline phase, making them comparable for a uniaxial stress state. The plastic rate of stretching is defined by an associated flow rule [18]. The Arruda-Boyce eight-chain network rubber elasticity model [41] is used to account for orientation induced hardening [42]. 


\subsubsection{Composite inclusion model}

The mechanical behavior at the macroscopic scale is modeled by an aggregate of layered two-phase composite inclusions, as proposed by Lee et al. [15, 16] for rigid-viscoplastic material behavior. The stress and deformation fields in each separate phase of the composite inclusion are assumed to be piecewise homogeneous; but they can be different between the two coupled phases. The inclusion-averaged Cauchy stress and the inclusion-averaged deformation gradient are given by the volume-average of the fields of the constituent phases. Furthermore, the crystalline lamella and the amorphous layer are assumed to be kinematically compatible and in equilibrium across the interface.

To relate the volume-averaged mechanical response of each layered composite inclusion to the imposed boundary conditions for an aggregate of inclusions, a hybrid local-global interaction law [15, 16, 18] is formulated. The consistency conditions for equilibrium and compatibility of the aggregate are maintained by enforcing the macroscopic stress and deformation to equal the volume-average of the stress and deformation fields of the composite inclusions.

In the hybrid interaction model, local-global compatibility conditions are formulated for the projections of the inclusion-averaged fields for which intra-inclusion equilibrium conditions were formulated. Inversely, local-global equilibrium conditions are assumed for the components of the inclusion-averaged fields that are subjected to intra-inclusion compatibility.

\subsubsection{Initial orientations}

The initial crystalline orientations of the oriented HDPE tapes with different draw ratios are generated according to the experimentally obtained pole figures of orientation distributions and Hermans' orientation distribution factors. For initially randomly oriented materials, possessing a spherulitic morphology, the angle between the chain direction $\vec{c}$ and the lamellar normal direction $\vec{n}^{I}$ is known to be $35^{\circ}$ [43, 44], corresponding to the $\{201\}$ planes. However, for oriented systems, the molecular chains are assumed to be more aligned with the lamellar normals. Shinozaki and Groves [45, 46] studied the structure of polyethylene and polypropylene sheets, oriented by solid state hot drawing at a temperature range of $105^{\circ} \mathrm{C}$ to $120^{\circ} \mathrm{C}$, after an elongation of approximately $600 \%$. The final material was observed to be oriented with molecular chains parallel to the initial draw direction, and the lamellae roughly perpendicular to the draw direction and, therefore, normal to the molecular chain axis. Their finding is also supported by experimental WAXS and SAXS data [47-49] on structural changes during hot drawing of semicrystalline polymers, which show a process of transformation of 
crystalline lamellae into much smaller folded-chain crystalline blocks, with both their crystalline lamellar normal and chain orientation almost parallel to the draw direction, which are connected by somewhat extended amorphous chains. In line with that, a reduced chain tilt angle of $5^{\circ}$, with lamellar surfaces again of the $\{h 0 l\}$ type, is assumed.

Figure 5.5 shows equal area projection pole figures of the principal crystallographic lattice directions together with the initial lamellar orientations. The oriented structure of the material is represented by an aggregate of 500 composite inclusions. The resulting Hermans' orientation distribution factors are listed in table 5.2, which shows a good agreement with experimentally obtained ones in table 5.1 .
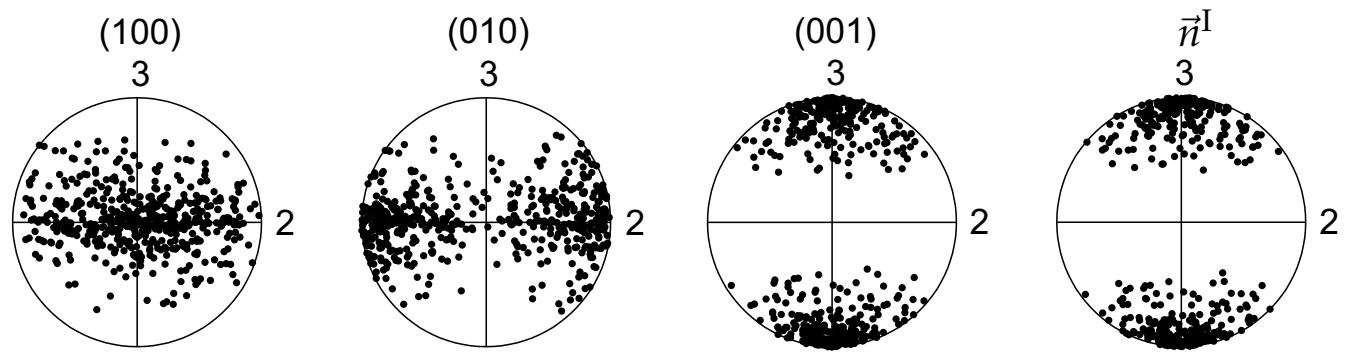

a
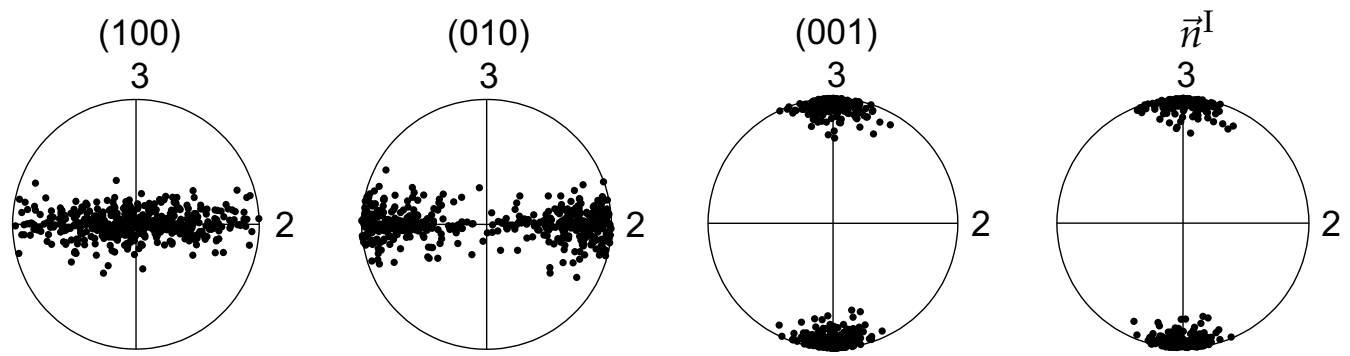

$\mathrm{b}$

Figure 5.5 Equal area projection pole figures of the principal crystallographic and lamellar orientation distributions for HDPE tape with draw ratios of 4 (a) and 6 (b). The draw direction is vertical.

The elasto-viscoplastic composite inclusion model is used to investigate the effect of the morphological factors, namely the tilt angle and the degree of crystallinity, on the mechanical behavior of the material. In the model, the elastic deformation of the interlamellar layer is characterized by the shear and bulk moduli, $G^{\mathrm{a}}$ and 
Table 5.2 Hermans'orientation distribution factors of crystallographic directions, based on generated orientations.

\begin{tabular}{ccccc}
\hline sample & draw ratio & $f_{a}$ & $f_{b}$ & $f_{c}$ \\
\hline 1 & 4 & -0.38 & -0.32 & 0.73 \\
2 & 6 & -0.47 & -0.47 & 0.94 \\
\hline
\end{tabular}

$K^{\mathrm{a}}$, respectively. The elastic parameters as well as the hardening properties of the amorphous phase are summarized in table 5.3, with $\mu_{\mathrm{R}}$ and $N$ being respectively the constants proportional to the initial stiffness and the number of rigid links between entanglements in the Arruda-Boyce eight-chain model [41]. For

Table 5.3 Elastic and hardening parameters of the amorphous phase.

\begin{tabular}{cccc}
\hline$G^{\mathrm{a}}[\mathrm{MPa}]$ & $K^{\mathrm{a}}[\mathrm{MPa}]$ & $\mu_{\mathrm{R}}[\mathrm{MPa}]$ & $N$ \\
\hline 65 & 3000 & 1 & 49 \\
\hline
\end{tabular}

orthorhombic PE crystals, the anisotropic fourth order elasticity tensor involves nine independent elastic constants. Their values are given in table 5.4.

Table 5.4 Elastic constants [GPa] for the crystalline phase of PE [50].

\begin{tabular}{ccccccccc}
\hline$C_{11}$ & $C_{22}$ & $C_{33}$ & $C_{12}$ & $C_{13}$ & $C_{23}$ & $C_{44}$ & $C_{55}$ & $C_{66}$ \\
\hline 7.99 & 9.92 & 315.92 & 3.28 & 1.13 & 2.14 & 3.62 & 1.62 & 3.19 \\
\hline
\end{tabular}

Figure 5.6 shows the crystallographic slip kinetics and the amorphous yield kinetics for the isotropic sample at $23^{\circ} \mathrm{C}$. The evaluation of the kinetics at the microscopic level was performed, taking into account the tensile kinetics of the HDPE tape with draw ratio $\lambda=1$ (isotropic). Table 5.5 summarizes the yield kinetics of all slip systems and the amorphous phase, together with their activation energies and the reference shear rates $\dot{\gamma}_{0, \text { ref }}^{*}$ characterized at a reference temperature $T_{\text {ref, }}$, for both relaxation processes. A more elaborated discussion on the contribution of two relaxation processes to the yield kinetics is given in chapter 4 . The temperature dependence of the reference shear rates can then be 


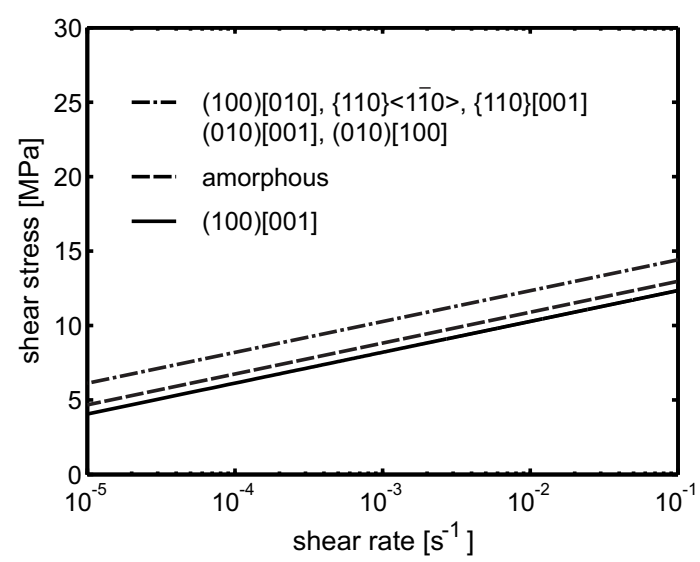

Figure 5.6 Crystallographic slip and amorphous yield kinetics, used to describe the yield kinetics of the isotropic sample at room temperature.

calculated using:

$$
\dot{\gamma}_{0}^{*}(T)=\dot{\gamma}_{0, \text { ref }}^{*} \exp \left[\frac{-\Delta U}{R}\left(\frac{1}{T}-\frac{1}{T_{\text {ref }}}\right)\right],
$$

with $T_{\text {ref }}=23^{\circ} \mathrm{C}$. The values of the characteristic shear stresses for the relaxation processes within the crystalline and amorphous phases in the Eyring model are set to $\tau_{0}^{\mathrm{c}, \mathrm{I}}=\tau_{0}^{\mathrm{a}, \mathrm{I}}=0.06 \mathrm{MPa}$ and $\tau_{0}^{\mathrm{c}, \mathrm{II}}=\tau_{0}^{\mathrm{a}, \mathrm{II}}=0.84 \mathrm{MPa}$.

A systematic study on the effect of the tilt angle as well as the degree of crystallinity on the model prediction of the tensile yield kinetics of the sample with draw ratio of 6, loaded in drawing direction, is illustrated in figure 5.7. As can be seen, the deformation kinetics is predominantly influenced by the chain tilt angle, whereas the crystallinity has a much smaller effect. Therefore the assumption of a volumetric degree of crystallinity of $60 \%$ for all samples would not drastically affect their deformation kinetics. Furthermore, the results are observed to not be much sensitive to making the chain tilt angle smaller than $5^{\circ}$.

\subsection{Results and discussion}

The mechanical response of HDPE tapes with various microstructures subjected to uniaxial tension is investigated here for a range of strain rates and loading angles, using the composite inclusion model. The starting point is formed 
Table 5.5 Yield mechanisms in the constituent phases, with the reference shear rates and activation energies of processes, corresponding to the viscoplastic Eyring flow rule, at $T_{\text {ref }}=23^{\circ} \mathrm{C}$.

\begin{tabular}{lcccc}
\hline & \multicolumn{2}{c}{ Process I } & \multicolumn{2}{c}{ Process II } \\
\hline Mechanism & $\dot{\gamma}_{0, \text { ref }}^{*}\left[\mathrm{~s}^{-1}\right]$ & $\Delta U^{\mathrm{I}}\left[\mathrm{kJ} \mathrm{mol}^{-1}\right]$ & $\dot{\gamma}_{0, \text { ref }}^{*}\left[\mathrm{~s}^{-1}\right]$ & $\Delta U^{\mathrm{II}}\left[\mathrm{kJ} \mathrm{mol}^{-1}\right]$ \\
\hline$(100)[001]$ & $8 \times 10^{-35}$ & 512 & $2 \times 10^{-5}$ & 105 \\
$(010)[001]$ & $2 \times 10^{-49}$ & 762 & $2 \times 10^{-5}$ & 105 \\
$\{110\}[001]$ & $2 \times 10^{-49}$ & 762 & $2 \times 10^{-5}$ & 105 \\
\hline$(100)[010]$ & $2 \times 10^{-49}$ & 762 & $2 \times 10^{-5}$ & 105 \\
$(010)[100]$ & $2 \times 10^{-49}$ & 762 & $2 \times 10^{-5}$ & 105 \\
$\{110\}\langle 1 \overline{1} 0\rangle$ & $2 \times 10^{-49}$ & 762 & $2 \times 10^{-5}$ & 105 \\
\hline amorphous & $6 \times 10^{-39}$ & 516 & $2 \times 10^{-5}$ & 105 \\
\hline
\end{tabular}

by characterization of the kinetics of the crystallographic slip systems and the deformation kinetics of the amorphous domain at the microscopic level to quantitatively predict the macroscopic plastic flow kinetics for the sample with draw ratio $\lambda=1$, which is both structurally and mechanically found to be isotropic. There are eight physically distinct slip systems present in the crystalline domain of polyethylene, which comprise six crystallographically different types of slip systems, each possibly having different properties. For an isotropic system, at the macroscopic level only the effective contribution of all these slip systems, combined with that of the amorphous domains is observed. It is, consequently, not possible to completely characterize and distinguish between the effect of different slip systems for such isotropic systems [51]. Therefore, the properties of some slip systems are assumed to be the same, see figure 5.6 .

\subsubsection{Isotropic amorphous domains}

The model predictions of the tensile yield kinetics of the HDPE tape with draw ratio $\lambda=1$ is shown in figure $5.8 \mathrm{a}$, and compared with experimental data. In both simulations and experiments, a similar strain rate dependence of the yield stress for various loading angles is found. Applying the same kinetics (figure 5.6), to the oriented samples, with draw ratios $\lambda=4$ and $\lambda=6$, gives the results shown in figures 5.8b and 5.8k, respectively. As can be seen, the model predictions 


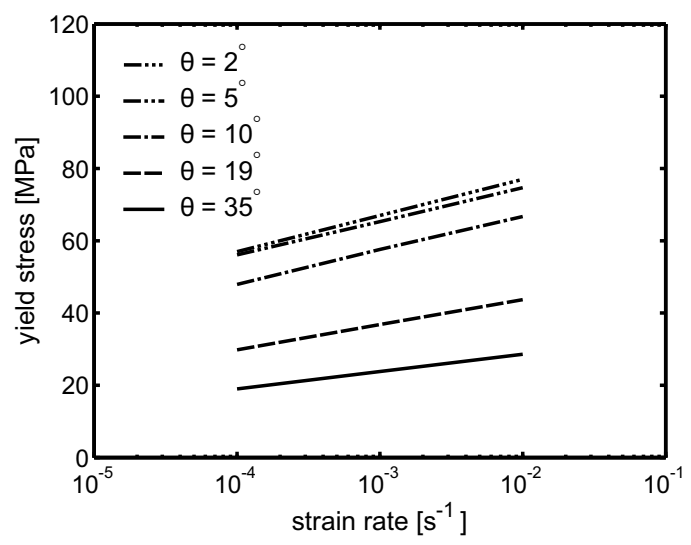

a

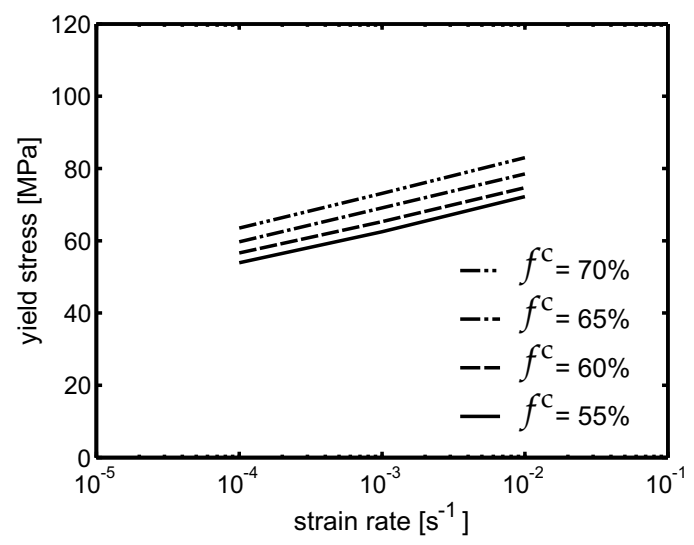

b

Figure 5.7 (a) The effect of varying chain tilt $\theta$ on the tensile yield kinetics for a constant crystallinity of $f^{c}=60 \%$. (b) The effect of varying crystallinity on the tensile yield kinetics for a constant chain tilt of $\theta=5^{\circ}$. The loading direction is aligned with the draw direction of the sample with draw ratio of 6 .

are reasonably good for a loading angle of $50^{\circ}$, whereas for the lower loading angles of $20^{\circ}$ and $0^{\circ}$ the kinetics is not predicted well. The results show that the anisotropy of the crystalline phase is not sufficient to capture the anisotropic macroscopic yield kinetics. This could be explained by the fact that in the solid state drawn tapes, the anisotropy is deformation-induced, causing not only the crystalline phase, but also the amorphous domains to stretch and orient in the drawing direction.

The results at a testing temperature of $80^{\circ} \mathrm{C}$ are shown in figure 5.9. It is observed that, although the $20^{\circ}$ and $50^{\circ}$ deformation kinetics are described reasonably well, the $0^{\circ}$ kinetics for both oriented samples is underestimated. This could be an indication that the influence of the oriented amorphous domains on the macroscopic flow kinetics, is present even at elevated temperatures.

\subsubsection{Oriented amorphous domains}

Bartczak et al. [30] studied the molecular orientation of the amorphous component in oriented semicrystalline high density polyethylene, produced by plane strain compression. Their results suggest that the plastic deformation induces a preferred orientation of macromolecules not only in the crystalline domain, but also for the amorphous component. They proposed that the oriented amorphous component consists of domains of extended chain segments, which are closely packed in a two-dimensional pseudo-hexagonal structure. The presence of an oriented amorphous domain in oriented semicrystalline polymers is also 


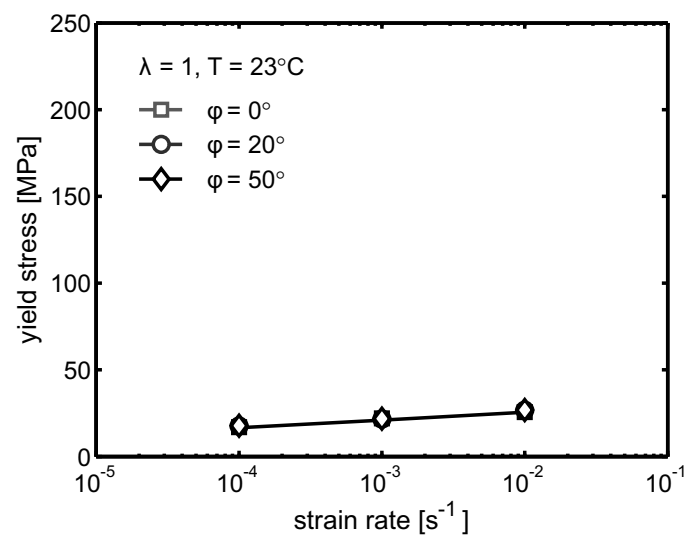

a

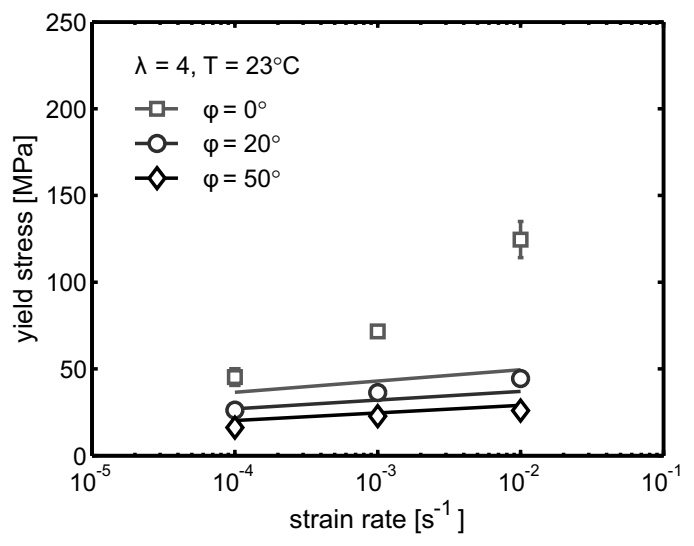

b

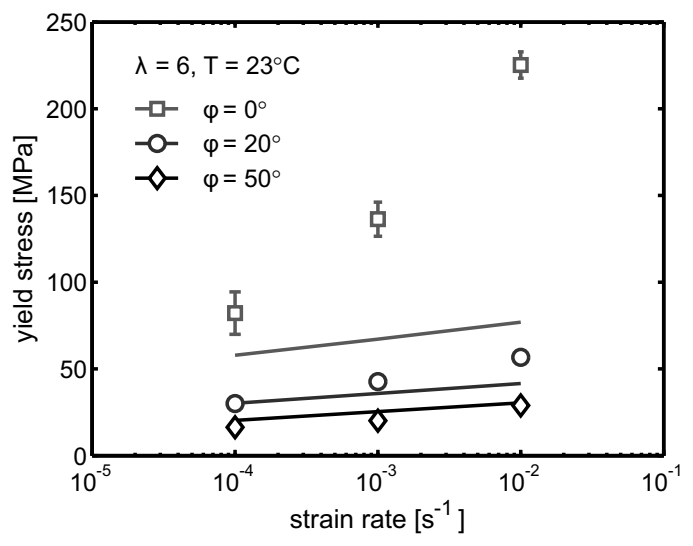

C

Figure 5.8 The model prediction of the tensile yield kinetics of the sample with (a) $\lambda=1$, (b) $\lambda=4$ and (c) $\lambda=6$, at various loading angles. Lines show the micromechanical model prediction and symbols indicate experimental data.

reported elsewhere [52].

First, the influence of the kinetics of the amorphous phase on the macroscopic yield kinetics is systematically probed. In order to just study the effect of the amorphous domain, the degree of crystallinity is set to be zero. The effective shear stress of the amorphous phase is related to the effective shear strain by an Eyring flow rule, which is characterized by two parameters, namely $\dot{\gamma}_{0}^{*}$ and $\tau_{0}$, see equation (5.4). The effect of these parameters on the macroscopic tensile yield kinetics is shown in figure 5.10, changing $\dot{\gamma}_{0}^{*}$ only affects the level of the yield stress, while $\tau_{0}$ mainly governs the slope. Therefore, a combination of these two parameters enables to describe a loading angle dependent yield kinetics for the amorphous phase. 


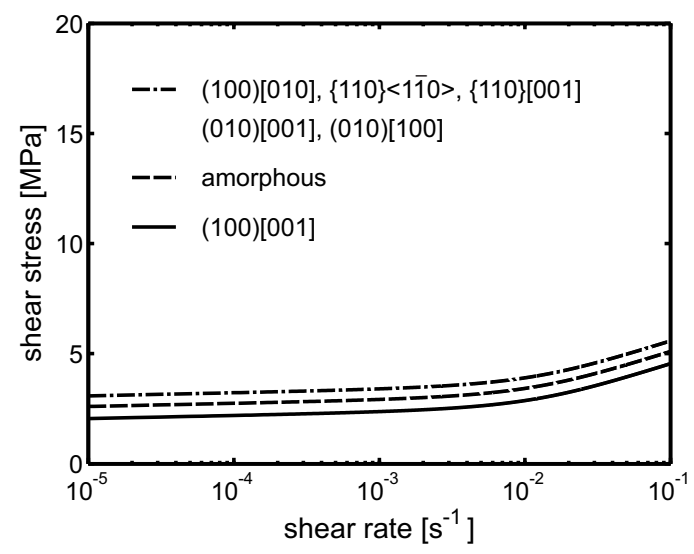

a

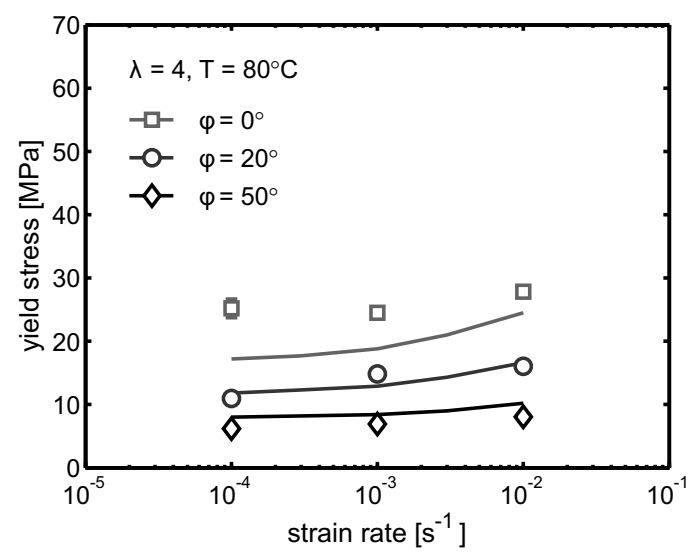

b

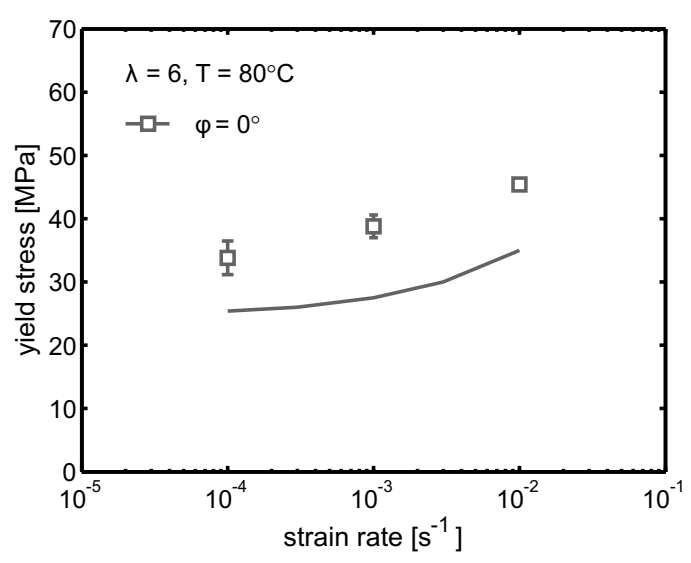

$\mathrm{C}$

Figure 5.9 (a) Crystallographic slip and amorphous yield kinetics at $80^{\circ} \mathrm{C}$, and the resulting micromechanical model prediction of the deformation kinetics of sample with (b) $\lambda=4$ and (c) $\lambda=6$. Lines show the micromechanical model prediction and symbols indicate experimental data.

The effect of introducing an oriented amorphous phase combined with the contribution of the anisotropic crystalline phase on the yield kinetics of the oriented samples is given in figure 5.11. The crystallographic slip kinetics are taken the same as the ones given in figure [5.6. To describe the macroscopic yield kinetics for every specified load angle, the kinetics of the amorphous phase is changed. The reference shear rates $\dot{\gamma}_{0 \text {,ref }}^{*}$ and the characteristic shear stresses $\tau_{0}$ used for process II in every load angle are summarized in table 5.6. The properties of process I are set to be the same as in table 5.5. A higher modulus is assumed for the $0^{\circ}$ loading angle, since the elastic properties also become anisotropic for oriented amorphous domains [52, 53]. As can be seen 


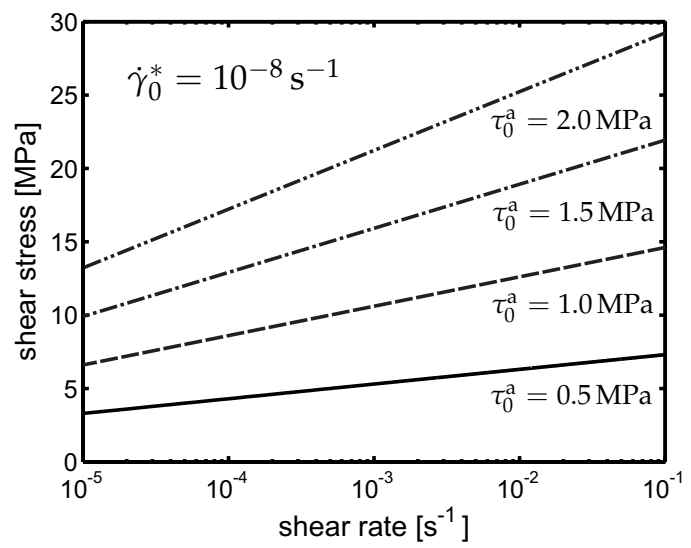

a

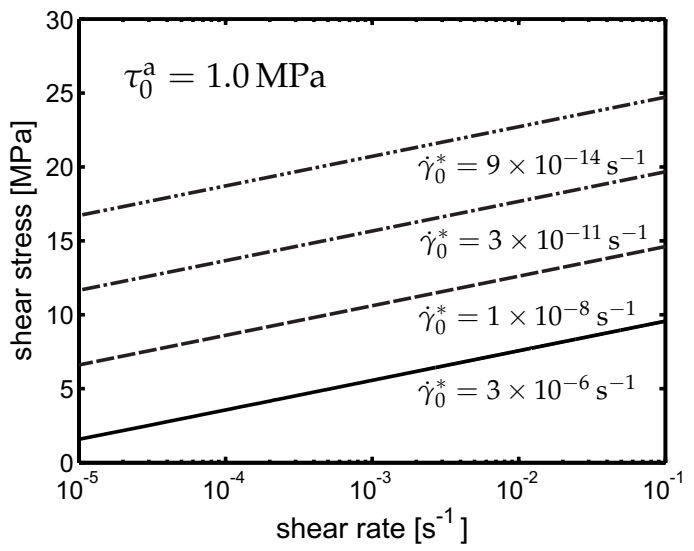

C

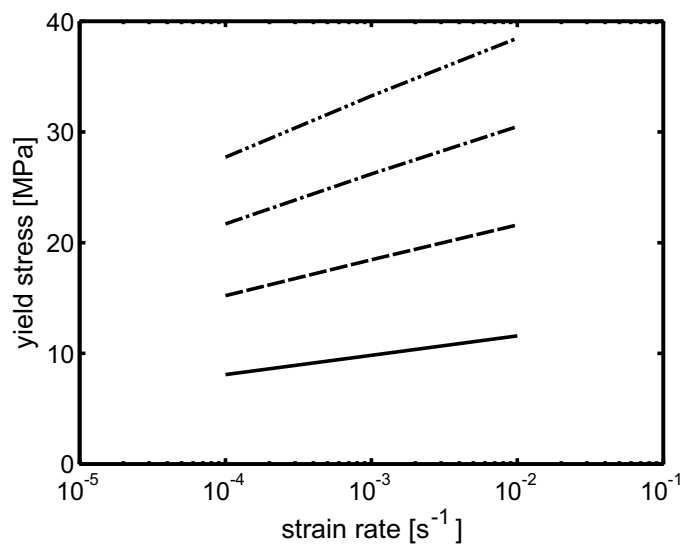

b

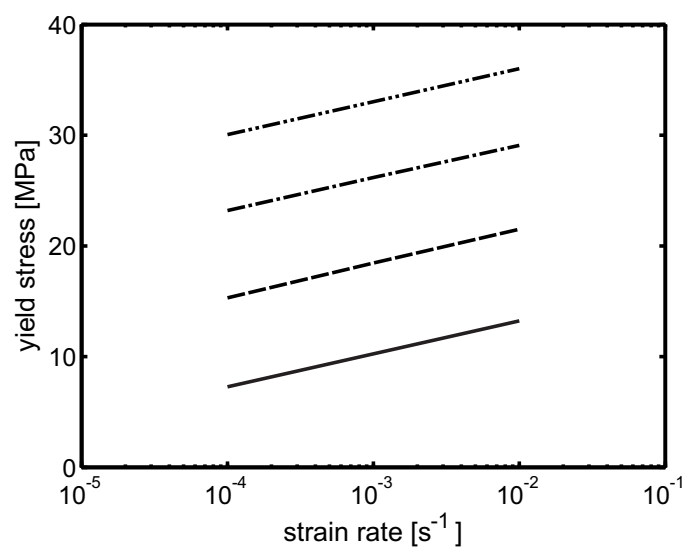

d

Figure 5.10 Influence of the varying characteristic shear stress $\tau_{0}$ (a) and reference shear rate $\dot{\gamma}_{0, \text { ref }}^{*}(\mathrm{c})$ of the amorphous phase (see equation (5.4)) on the macroscopic yield kinetics (b) and (d).

in figure 5.11, by assuming a loading angle dependent yield kinetics for the amorphous phase of the oriented HDPE tapes, it is possible to describe the deformation kinetics of two tapes with different degrees of orientation at various loading angles. However, it should be noted that for the oriented sample with $\lambda=4$, the $0^{\circ}$ yield kinetics is not predicted well, especially at high strain rates. The values for the amorphous yield kinetics used are restricted, and not allowed to be higher than those of the sample with $\lambda=6$, since the sample with $\lambda=4$ is less oriented than the one with $\lambda=6$. The low model prediction could partly be due to the effect of the initial orientation distribution. The generated initial orientations were quantified by the experimentally obtained Hermans' orientation factor. There is not a unique solution for a Hermans' orientation factor of approximately 0.7 and different distributions can result in the same number. 
Table 5.6 The reference shear rates and characteristic shear stresses of the amorphous phase for varying loading angles at $T_{\text {ref }}=23^{\circ} \mathrm{C}$.

\begin{tabular}{ccccc}
\hline & \multicolumn{2}{c}{ Sample $\lambda=4$} & \multicolumn{2}{c}{ Sample $\lambda=6$} \\
\hline Loading angle & $\dot{\gamma}_{0, \text { ref }}^{*}\left[\mathrm{~s}^{-1}\right]$ & $\tau_{0}^{\mathrm{a}}[\mathrm{MPa}]$ & $\dot{\gamma}_{0, \text { ref }}^{*}\left[\mathrm{~s}^{-1}\right]$ & $\tau_{0}^{\mathrm{a}}[\mathrm{MPa}]$ \\
\hline $0^{\circ}$ & $1.6 \times 10^{-4}$ & 6.3 & $1.9 \times 10^{-4}$ & 7.7 \\
$20^{\circ}$ & $5.1 \times 10^{-5}$ & 1.4 & $1.2 \times 10^{-4}$ & 3.4 \\
$50^{\circ}$ & $2.0 \times 10^{-5}$ & 0.84 & $2 \times 10^{-5}$ & 0.84 \\
\hline
\end{tabular}

The results show that the prediction of anisotropic yield kinetics with high values of yield stress for a $0^{\circ}$ load angle, requires the description of anisotropic yield kinetics for the amorphous domain, due to the presence of oriented extended chains. The need of describing an anisotropic behavior of the oriented amorphous phase is also supported by experimental data for the loading angle dependence of the elastic modulus of the oriented tapes, as shown in figure 5.12 , where a loading angle dependent Young's modulus is observed. Predictions of the elastic $\hat{\epsilon}$-inclusion model, introduced in chapter 2 , are given for various properties of the crystalline and amorphous domains, including an orthorhombic material behavior for the amorphous phase, which is characterized by the following set of parameters:

$$
\left\{\begin{array}{l}
E_{11}=E_{22}=4.5 \mathrm{MPa}, E_{33}=450 \mathrm{MPa} \\
\frac{1}{2}\left(v_{12}+v_{13}\right)=\frac{1}{2}\left(v_{21}+v_{23}\right)=\frac{1}{2}\left(v_{31}+v_{32}\right)=v=0.4996 \\
G_{12}=G_{13}=G_{23}=\frac{E_{11}}{2(1+v)}=1.5 \mathrm{MPa}
\end{array}\right.
$$

It is observed that a better prediction is obtained, when an anisotropic (orthorhombic) behavior is assumed for the amorphous phase, with a high elastic modulus in the direction of the chains in the neighboring crystalline lamella. A better prediction for the elastic modulus close to a loading angle of $90^{\circ} \mathrm{can}$ be achieved by using the experimentally obtained elastic constants [54] for the crystalline phase, which are lower than the theoretically calculated values [50], see chapter 2. A lower stiffness could likely be due to the increased effect of imperfections such as dislocations or defects within the crystal during plastic deformation [52]. 


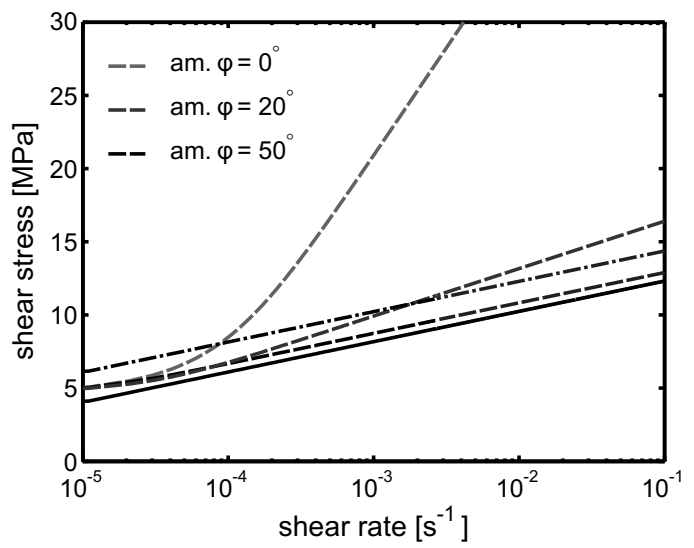

a

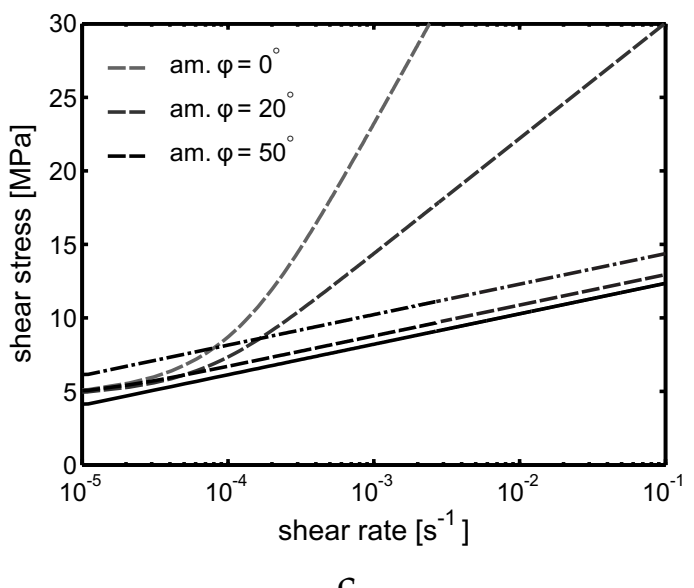

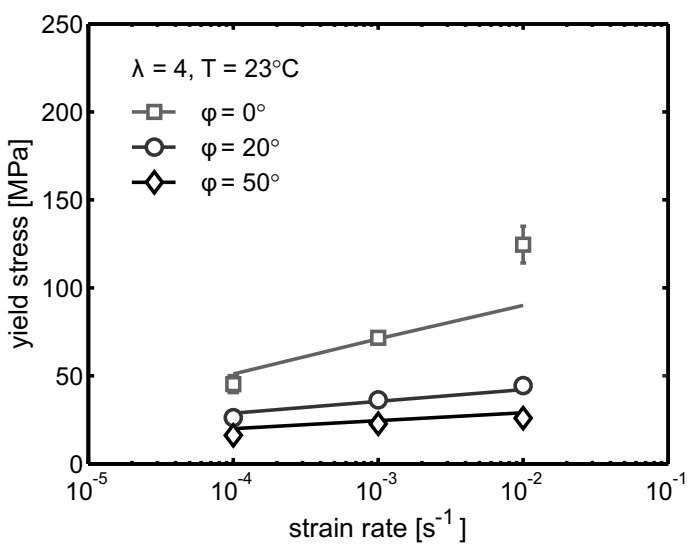

b

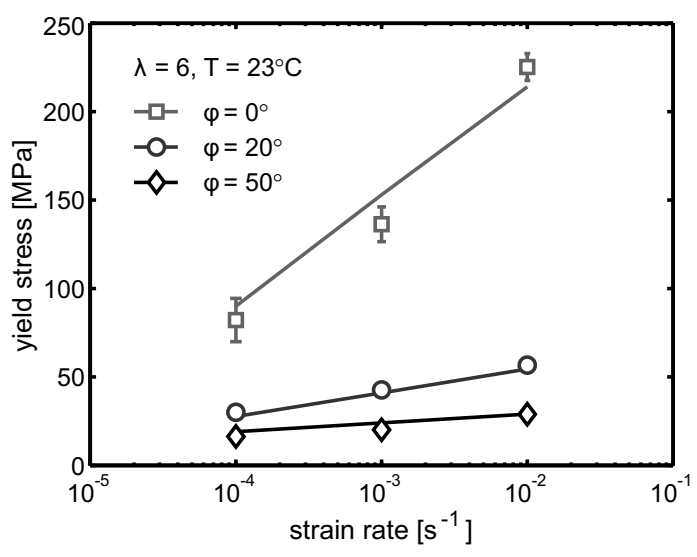

d

Figure 5.11 (a) Crystallographic slip and varying amorphous yield kinetics (table 5.6), used to describe the yield kinetics of the sample with $\lambda=4$ at room temperature. (b) Micromechanical model prediction of the anisotropic yield kinetics of the sample with $\lambda=4$. (c) Crystallographic slip and varying amorphous yield kinetics (table 5.6), used to describe the yield kinetics of the sample with $\lambda=6$ at room temperature. (d) Micromechanical model prediction of the anisotropic yield kinetics of the sample with $\lambda=6$. Lines show the micromechanical model prediction and symbols indicate experimental data. Slip kinetics are the same as in figure 5.6, with the solid line showing the kinetics of (100)[001] chain slips, and the dash-dot line denoting the remaining kinetics.

\subsubsection{Probing characterization of crystallographic slip systems}

It is not possible to distinguish between the contribution of different slip systems for an isotropic semicrystalline polymer [51], since the macroscopic plastic flow is governed by the effective contribution of different types of slip systems with different properties. Therefore, it was suggested that more experimental data 


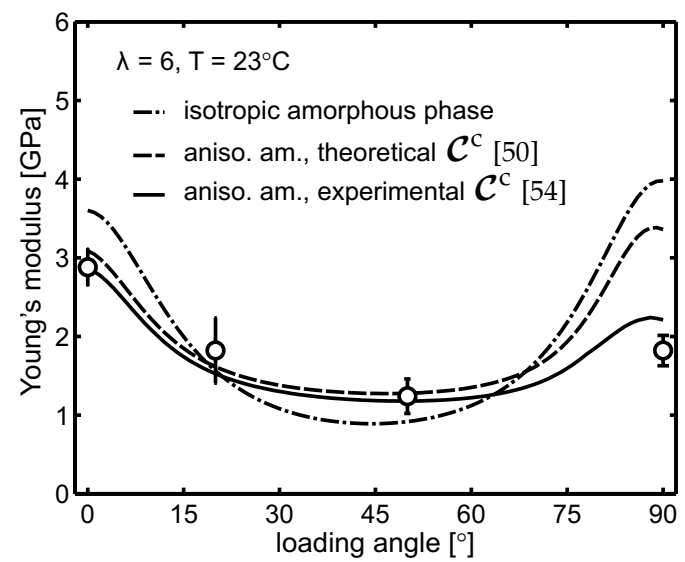

Figure 5.12 Young's modulus of the oriented sample with $\lambda=6$ versus the loading angle. Symbols denote experimental data, and lines show the prediction of the elastic $\hat{\epsilon}$ inclusion model introduced in chapter 2, The elastic constants for the theoretically and experimentally obtained stiffness of the crystalline phase are given in table 2.1]

of a system with an initially oriented morphology are needed to correctly make a distinction between different types of slip systems and to characterize them. However, the results of micromechanical modeling of oriented HDPE tapes in section 5.4.2 show that the deformation kinetics is dominantly influenced by the properties of the oriented amorphous phase. This is due to the fact that in solid state drawing, both crystalline and amorphous domains are oriented. Therefore, to identify the properties of different crystallographic slip systems, samples with oriented crystalline lamellae and unoriented amorphous domains are needed in combination with the micromechanical model.

Figure 5.13 illustrates the resolved shear stress of a selected number of slip systems at the engineering yield stress (maximum in local stress) under a constant applied strain rate of $10^{-3} \mathrm{~s}^{-1}$ for the sample with $\lambda=6$ in tension at $23^{\circ} \mathrm{C}$, for various loading angles. In these pole figures, the location of markers shows the loading direction relative to the crystallographic axes of each lamella and colors represent the magnitude of the resolved shear stress of the specified slip system for each inclusion. As can be seen, for a $0^{\circ}$ load angle, the stress is mainly resolved on the chain slip systems (100)[001] and $\{110\}[001]$, whereas the amount of resolved shear stress on the transverse slip systems (100)[010] and $\{110\}\langle 1 \overline{1} 0\rangle$ is less significant. As the load angle increases to $90^{\circ}$, a change in the shear stress on the slip systems is observed. At a $90^{\circ}$ load angle, the stress is mostly resolved on the (100)[010] and $\{110\}\langle 110\rangle$ transverse slip systems, while in this case the (100)[001] and $\{110\}[001]$ chain slip systems are almost inactive. The effect of the properties of different groups of slip systems on 
(a)
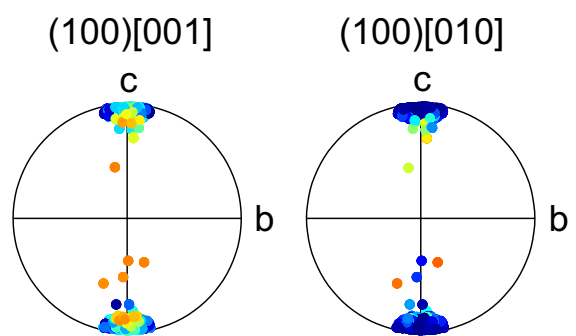

$\{110\}<1 \overline{10}>$

$\{110\}[001]$

[MPa]
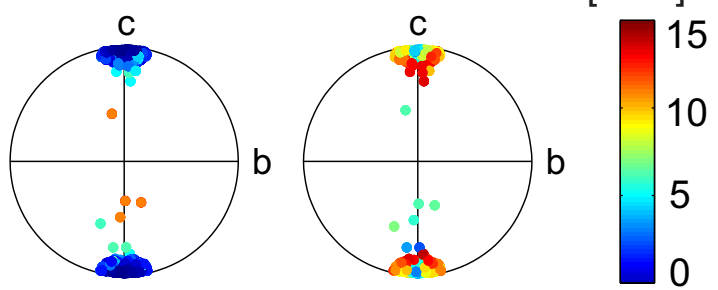

(100)[001]

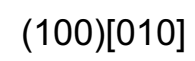

$\{110\}<1 \overline{1} 0>$

$\{110\}[001]$

[MPa]
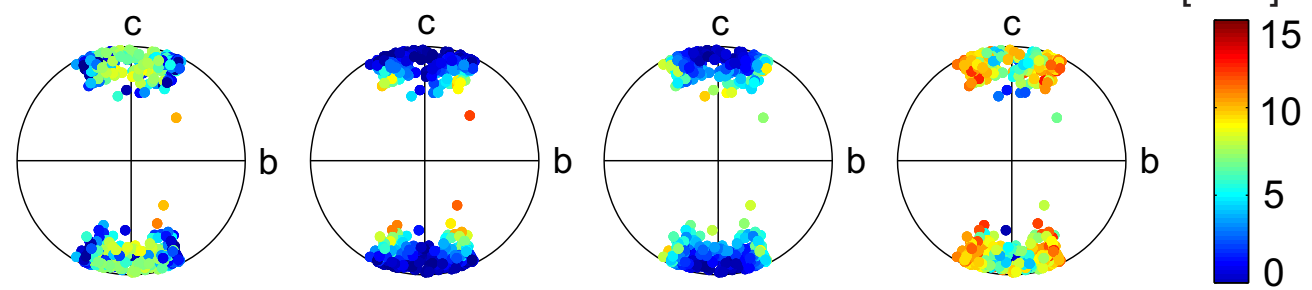

(100)[001]

(100)[010]

$\{110\}<1 \overline{1} 0>$

$\{110\}[001]$
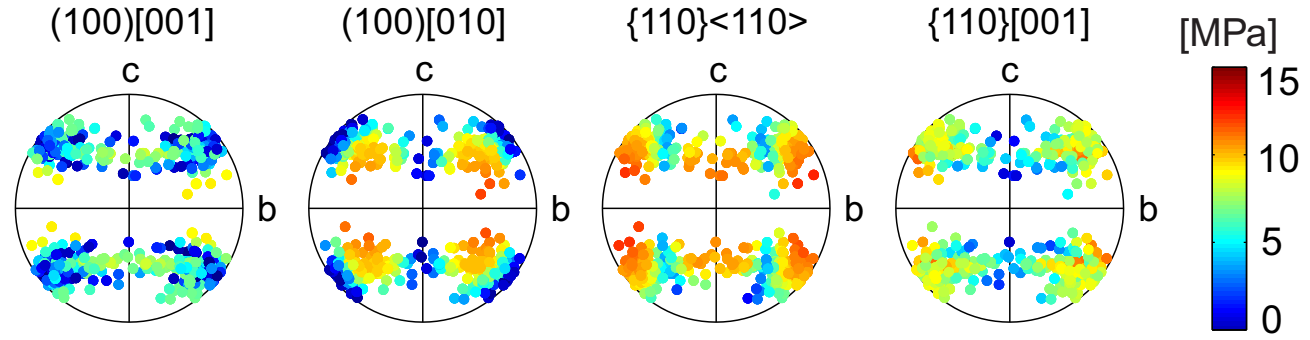

(100)[001]

(100)[010]

$\{110\}<1 \overline{1} 0>$

$\{110\}[001]$

[MPa]
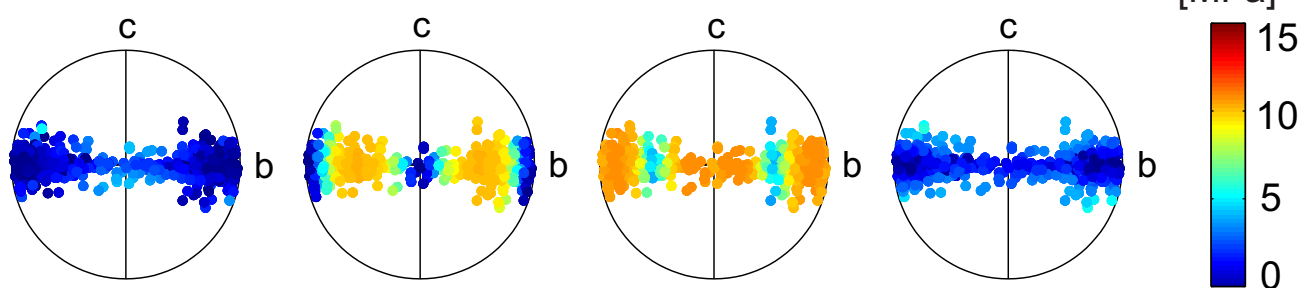

Figure 5.13 Inverse pole figures showing the resolved shear stress of a number of slip systems at the engineering yield stress under a constant strain rate of $10^{-3} \mathrm{~s}^{-1}$ in tension at $23^{\circ} \mathrm{C}$, with various load angles of (a) $0^{\circ}$, (b) $20^{\circ}$, (c) $50^{\circ}$ and (d) $90^{\circ}$, for the sample with $\lambda=6$. The kinetics are the same as in figure 5.6 Colors represent the magnitude of the resolved shear stress of the specified slip system for each inclusion, and the location of the markers indicates the loading direction relative to the crystallographic axes of each lamella. 
the macroscopic deformation kinetics is shown in figure 5.14. The results are compared to those corresponding to the slip and amorphous yield kinetics of figure 5.6, as a reference to indicate the effect of different slip systems. First the level of the chain slip kinetics is increased, while the level of the transverse slip kinetics is the same as in figure 5.6, see figure 5.14 $\mathrm{a}$. The effect on the macroscopic yield kinetics is depicted in figure $5.14 \mathrm{~b}$, which shows an increase in the $0^{\circ}$ yield kinetics, whereas the $90^{\circ}$ yield kinetics does not change. In the same way, by increasing the level of the transverse slip systems, while keeping the chain slip systems the same (figure 5.14 $\mathrm{c}$ ), the $90^{\circ}$ yield kinetics increases, whereas the $0^{\circ}$ yield kinetics remain almost the same, as shown in figure 5.14 $\mathrm{d}$. These results are supported by the inverse pole figures showing the resolved shear stress of slip systems in figure 5.13 ,

Alternative experimental techniques to obtain oriented samples, with a somewhat isotropic amorphous fraction could be injecting molding [55] and extended dilatometry $(\mathrm{PVT}-\dot{\gamma})$ [56, 57]. With these techniques, the crystalline orientation is due to the flow induced orientation of the melt, and the presence of high temperature leads an almost relaxed and isotropic amorphous phase, making the characterization of slip kinetics using the micromechanical model possible.

\subsection{Conclusions}

The deformation of semicrystalline polymers is the result of the interplay of various mechanisms at different levels. A quantitative and accurate prediction of their mechanical behavior requires a coupled and detailed modeling of the various deformation mechanisms in the heterogeneous microstructure. A multi-scale micromechanical model is used to describe the deformation kinetics of hot-drawn oriented semicrystalline polyethylene tapes with different initial microstructures. The model represents the microstructure as an aggregate of layered composite inclusions, each consisting of a crystalline lamella, which is mechanically coupled to its adjacent amorphous layer. The crystalline phase is modeled as an anisotropic elastic material with plastic flow governed by crystallographic slip. The amorphous phase is assumed to be isotropic elastic with a rate dependent plastic flow and strain hardening resulting from molecular orientation. To relate the volume-averaged mechanical behavior of each layered composite inclusion to the aggregate of composite inclusions, a hybrid localglobal interaction law is used.

The initial orientation distribution for the micromechanical model was generated based on pole figures from wide-angle $X$-ray diffraction experiments. A key issue in the initial morphology of oriented HDPE was found to be the angle between 


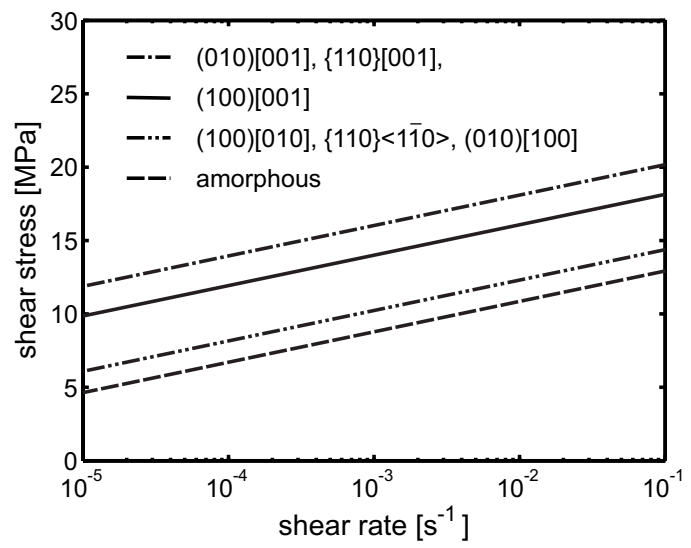

a

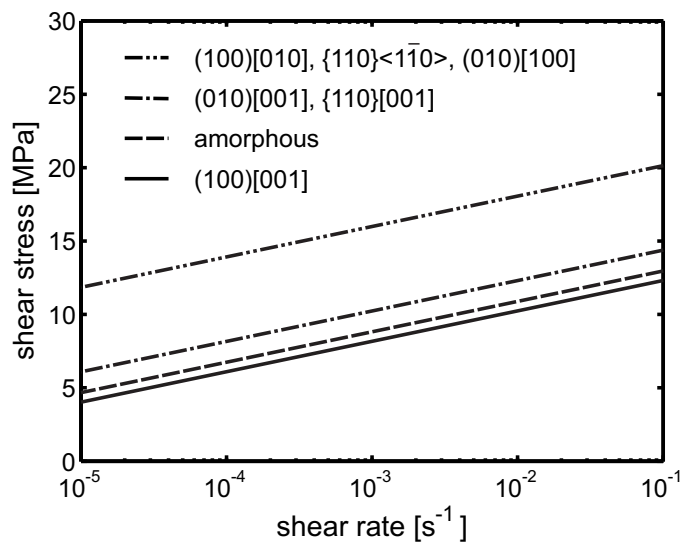

C

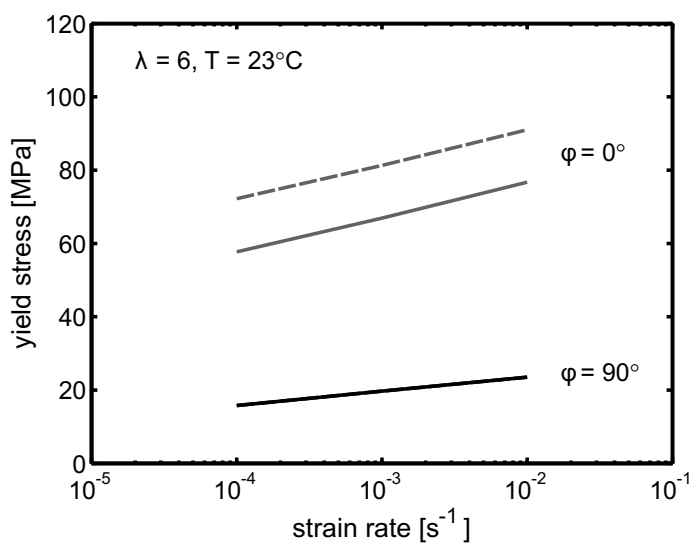

b

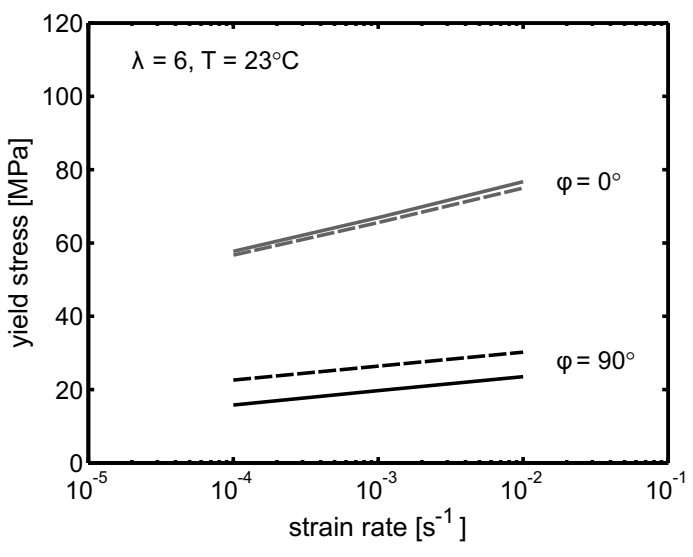

d

Figure 5.14 Crystallographic slip and amorphous yield kinetics with (a) increased chain slip kinetics and (c) increased transverse slip kinetics, and (b) and (d) the effect on the yield stress for different loading angles. Solid lines show the model prediction corresponding to the kinetics of (a) and (c), and dashed lines give the prediction corresponding to the kinetics of figure 5.6 .

the chain direction and the lamellar normal direction, also referred to as chain tilt, which showed a strong influence on the yield kinetics.

The results of micromechanical model with isotropic yield kinetics of the amorphous phase showed that the orientation of the crystalline phase alone is not sufficient to account for the anisotropic yield kinetics. The additional contribution of the anisotropic yield kinetics of the interlamellar material is needed to quantitatively describe the anisotropic mechanical response of the tapes. This is due to the fact that the anisotropy of the solid state drawn tapes is deformationinduced, which means that not only the crystalline phase, but also the chains in the amorphous domains are oriented. To investigate the effect of an oriented 
amorphous phase on the macroscopic plastic flow kinetics, a loading angle dependent yield kinetics for the amorphous phase was taken, which enabled to describe the deformation kinetics of the oriented tapes at various loading angles. Therefore, a critical factor for prediction of the anisotropic mechanical response of oriented HDPE tapes is the dominant influence of the yield kinetics of the amorphous phase on the macroscopic yield kinetics, and an accurate description of the constitutive behavior of the oriented amorphous phase is required.

Finally, the possibilities for characterizing the properties of distinct crystallographic slip systems were investigated. The results of the inverse pole figures showing the resolved shear stress of a number of slip systems revealed the feasibility of distinguishing between the contribution of different slip systems for various loading angles. A combination of experimental data of oriented samples with isotropic amorphous phase, and the micromechanical model seems to be promising for characterization of different crystallographic slip systems. 


\section{References}

[1] G. Meinel, N. Riorosoff, and A. Peterlin. Plastic deformation of polyethylene. I. Change of morphology during drawing of polyethylene of high density. Journal of Polymer Science, Part A-2 8:1723-1740, 1970.

[2] P.B. Bowden and R.J. Young. Deformation mechanisms in crystalline polymers. Journal of Materials Science 9:2034-2051, 1974.

[3] R. Popli and L. Mandelkern. Influence of structural and morphological factors on the mechanical properties of the polyethylenes. Journal of Polymer Science, Part B: Polymer Physics 25:441-483, 1987.

[4] N.S.J.A. Gerrits and Y. Tervoort. Deformation mechanisms during uniaxial drawing of meltcrystallized ultra-high molecular weight polyethylene. Journal of Material Science 25:13851390, 1992.

[5] E.F. Oleinik. Plasticity of semicrystalline flexible-chain polymers at the microscopic and mesoscopic levels. Polymer Science Series C 45:17-117, 2003.

[6] C. G'sell and A. Dahoun. Evolution of microstructure in semi-crystalline polymers under large plastic deformation. Materials Science and Engineering: A 175:183-199, 1994.

[7] C. G'sell, V. Favier, J.M. Hiver, A. Dahoun, M.J. Philippe, and G.R. Canova. Microstructure transformation and stress-strain behavior of isotactic polypropylene under large plastic deformation. Polymer Engineering \& Science 37:1702-1711, 1997.

[8] A. Galeski. Strength and toughness of crystalline polymer systems. Progress in Polymer Science 28:1643-1699, 2003.

[9] R.J. Young. A dislocation model for yield in polyethylene. Philosophical Magazine 30, 1974.

[10] R.J. Young. Screw dislocation model for yield in polyethylene. Materials forum 11:210-216, 1988.

[11] L.G. Shadrake and F. Guiu. Dislocations in polyethylene crystals: line energies and deformation modes. Philosophical Magazine 34:565-581, 1976.

[12] R. Séguéla, V. Gaucher-Miri, and S. Elkoun. Plastic deformation of polyethylene and ethylene copolymers: Part I. Homogeneous crystal slip and molecular mobility. Journal of Material Science 33:1273-1279, 1998.

[13] R. Séguéla. Dislocation approach to the plastic deformation of semicrystalline polymers: kinetic aspects for polyethylene and polypropylene. Journal of Polymer Science, Part B: Polymer Physics 40:593-601, 2002.

[14] D.M. Parks and S. Ahzi. Polycrystalline plastic deformation and texture evolution for crystals lacking five independent slip systems. Journal of the Mechanics and Physics of Solids 38:701-724, 1990.

[15] B.J. Lee, D.M. Parks, and S. Ahzi. Micromechanical modeling of large plastic deformation and texture evolution in semicrystalline polymers. Journal of the Mechanics and Physics of Solids 41:1651-1687, 1993.

[16] B.J. Lee, A.S. Argon, D.M. Parks, S. Ahzi, and Z. Bartczak. Simulation of large strain plastic deformation and texture evolution in high density polyethylene. Polymer 34:3555-3575, 1993. 
[17] B.J. Lee, S. Ahzi, and R.J. Asaro. On the plasticity of low symmetry crystals lacking 5 independent slip systems. Mechanics of Materials 20:1-8, 1995.

[18] J.A.W. van Dommelen, D.M. Parks, M.C. Boyce, W.A.M Brekelmans, and F.P.T Baaijens. Micromechanical modeling of the elasto-viscoplastic behavior of semi-crystalline polymers. Journal of the Mechanics and Physics of Solids 51:519-541, 2003.

[19] J.A.W. van Dommelen, D.M. Parks, M.C. Boyce, W.A.M Brekelmans, and F.P.T Baaijens. Micromechanical modeling of intraspherulitic deformation of semicrystalline polymers. Polymer 44:6089-6101, 2003.

[20] J.A.W. van Dommelen, B.A.G. Schrauwen, L.C.A. van Breemen, and L.E. Govaert. Micromechanical modeling of the tensile behavior of oriented polyethylene. Journal of Polymer Science, Part B: Polymer Physics 42:2983-2994, 2004.

[21] A. Sedighiamiri, L.E. Govaert, and J.A.W. van Dommelen. Micromechanical modeling of the deformation kinetics of semicrystalline polymers. Journal of Polymer Science, Part B: Polymer Physics 49:1297-1310, 2011.

[22] A. Keller and J.G. Rider. On the tensile behaviour of oriented polyethylene. Journal of Materials Science 1:389-398, 1966.

[23] N. Brown, R.A. Duckett, and I.M. Ward. The yield behaviour of oriented polyethylene terephthalate. Philosophical Magazine 18:483-502, 1968.

[24] I.M. Ward. Review: The yield behaviour of polymers. Journal of Materials Science 6:13971417, 1971.

[25] T.H. Yu and G.L. Wilkes. Orientation determination and morphological study of high density polyethylene (HDPE) extruded tubular films: effect of processing variables and molecular weight distribution. Polymer 37:4675-4687, 1996.

[26] T.H. Yu and G.L. Wilkes. Erratum: Orientation determination and morphological study of high density polyethylene (HDPE) extruded tubular films: effect of processing variables and molecular weight distribution. Polymer 38:1503-1505, 1997.

[27] H. Zhou and G.L. Wilkes. Orientation-dependent mechanical properties and deformation morphologies for uniaxially melt-extruded high-density polyethylene films having an initial stacked lamellar texture. Journal of Materials Science 33:287-303, 1998.

[28] B.A.G. Schrauwen, L.C.A. van Breemen, A.B. Spoelstra, L.E. Govaert, G.W.M. Peters, and H.E.H. Meijer. Structure, Deformation, and Failure of Flow-Oriented Semicrystalline Polymers. Macromolecules 37:8618-8633, 2004.

[29] D.W. van Krevelen. Properties of polymers. Elsevier, 1990.

[30] Z. Bartczak, A. Galeski, A.S. Argon, and R.E. Cohen. On the plastic deformation of the amorphous component in semicrystalline polymers. Polymer 37:2113-2123, 1996.

[31] P.H. Hermans, J.J. Hermans, D. Vermaas, and A. Weidinger. Deformation mechanism of cellulose gels. IV. General relationship between orientation of the crystalline and that of the amorphous portion. Journal of Materials Science 3:1-9, 1948.

[32] Z. Bartczak, R.E. Cohen, and A.S. Argon. Evolution of the crystalline texture of high density polyethylene during uniaxial compression. Macromolecules 25:4692-4704, 1992.

[33] Z. Bartczak, A.S. Argon, and R.E. Cohen. Deformation mechanisms and plastic resistence in single crystal textured high density polyethylene. Macromolecules 25:5036-5053, 1992. 
[34] L. Lin and A.S. Argon. Structure and plastic deformation of polyethylene. Journal of Materials Science 29:294-323, 1994.

[35] T. Ree and H. Eyring. Theory of non-Newtonian flow. I. Solid plastic system. Journal of Applied Physics 26:793-800, 1955.

[36] Z. Bartczak and A. Galeski. Plasticity of semicrystalline polymers. Macromolecular Symposia 294:67-90, 2010.

[37] B. Crist, C.J. Fisher, and P.R. Howard. Mechanical properties of model polyethylenes: tensile elastic modulus and yield stress. Macromolecules 22:1709-1718, 1989.

[38] R.H. Boyd. Relaxation processes in crystalline polymers: experimental behaviour - a review. Polymer 26:323-347, 1985.

[39] K. Schmidt-Rohr and H.W. Spiess. Chain diffusion between crystalline and amorphous regions in polyethylene detected by 2D exchange carbon-13 NMR. Macromolecules 24:5288$5293,1991$.

[40] R.H. Boyd. Relaxation processes in crystalline polymers: molecular interpretation - a review. Polymer 26:1123-1133, 1985.

[41] E.M. Arruda and M.C. Boyce. A three-dimensional constitutive model for the large stretch behavior of rubber elastic materials. Journal of the Mechanics and Physics of Solids 41:389-412, 1993.

[42] M.C. Boyce, D.M. Parks, and A.S. Argon. Large inelastic deformation of glassy polymers. part I: rate dependent constitutive model. Mechanics of Materials 7:15-33, 1988.

[43] D.C. Basset and A.M. Hodge. On the morphology of melt-crystallized polyethylene I. Lamellar profiles. Proceedings of the Royal Society of London A 377:25-37, 1981.

[44] S. Gautam, S. Balijepalli, and G.C. Rutledge. Molecular simulations of the interlamellar phase in polymers: effect of chain tilt. Macromolecules 33:9136-9145, 2000.

[45] D. Shinozaki and G.W. Groves. The plastic deformation of oriented polypropylene: tensile and compressive yield criteria. Journal of Materials Science 8:71-78, 1973.

[46] D.M. Shinozaki and G.W. Groves. The plastic deformation of oriented polypropylene and polyethylene: deformation mechanisms. Journal of Materials Science 8:1012-1022, 1973.

[47] A. Peterlin and F.J. Balta-Calleja. Diffraction studies of plastically deformed polyethylene. IV. Branched polyethylene. Colloid \& Polymer Science 242:1093-1102, 1970.

[48] N.A.J.M. Aerle and A.W.M. Braam. A structural study on solid state drawing of solutioncrystallized ultra-high molecular weight polyethylene. Journal of Materials Science 23:44294436, 1988.

[49] T. Sakurai, Y. Nozue, T. Kasahara, K. Mizunuma, N. Yamaguchi, K. Tashiro, and Y. Amemiya. Structural deformation behavior of isotactic polypropylene with different molecular characteristics during hot drawing process. Polymer 46:8846-8858, 2005.

[50] K. Tashiro, M. Kobayashi, and H. Tadakoro. Calculation of the three-dimensional elastic constants of polymer crystals. 2. Application to orthorhombic polyethylene and poly(vinyl alcohol). Macromolecules 11:914-918, 1978.

[51] A. Sedighiamiri, L.E. Govaert, M.J.W. Kanters, and J.A.W. van Dommelen. Micromechanics of semicrystalline polymers: Yield kinetics and long-term failure. Journal of Polymer Science Part B: Polymer Physics submitted, 2012. 
[52] S. Srinivas, P. Brant, Y. Huang, and D.R. Paul. Structure and properties of oriented polyethylene films. Polymer Engineering \& Science 43:831-849, 2003.

[53] G. Capaccio and I.M. Ward. Preparation of ultra-high modulus linear polyethylenes; effect of molecular weight and molecular weight distribution on drawing behaviour and mechanical properties. Polymer 15:233-238, 1974.

[54] C.L. Choy and W.P. Leung. Elastic moduli of ultradrawn polyethylene. Journal of Polymer Science: Polymer Physics Edition 23:1759-1780, 1985.

[55] T.B. van Erp, L.E. Govaert, and G.W.M. Peters. Mechanical Performance of Injection Molded Polypropylene: Characterization and Modeling. Macromolecular Materials and Engineering accepted, 2012.

[56] M.H.E. van der Beek, G.W.M. Peters, and H.E.H. Meijer. A dilatometer to measure the influence of cooling rate and melt shearing on specific volume. International Polymer Processing 20:111-120, 2005.

[57] R. Forstner, G.W.M. Peters, C. Rendina, J.W. Housmans, and H.E.H. Meijer. Volumetric rheology of polymers. The influence of shear flow, cooling rate, and pressure on the specific volume of iPP and P/E random copolymers. Journal of Thermal Analysis and Calorimetry 98:683-691, 2009. 



\title{
Conclusions and recommendations
}

Chapter 6

\begin{abstract}
The main ideas, methods and conclusions concerning the micromechanical modeling of semicrystalline polymers are summarized. Recommendations for future work are given.
\end{abstract}

\subsection{Conclusions}

The process of plastic deformation in semicrystalline polymers is complex due to their hierarchical structure. Any macroscopically homogeneous deformation is accommodated by a variety of competing phenomena and mechanisms at different levels. The mechanical properties strongly depend on morphological features (like degree of crystallinity, lamellar thickness and molecular weight), initial microstructure, as well as the evolution of the microstructure during plastic deformation. In line with that, this thesis aimed at establishing a quantitative relation between the microstructure and the mechanical performance of semicrystalline polymers, as characterized by elasto-viscoplastic deformation. In order to do that, a micromechanically based constitutive model was used. The model represents the microstructure as an aggregate of layered composite inclusions, each consisting of a crystalline lamella, which is mechanically coupled to its adjacent amorphous layer. The crystalline phase was modeled as anisotropic elastic with plastic flow governed by crystallographic slip. The amorphous phase was assumed to be isotropic elastic with a rate dependent plastic flow and strain hardening resulting from molecular orientation. To relate the volume-averaged mechanical response of each layered composite inclusion to the boundary conditions imposed, for an aggregate of composite inclusions, a hybrid local-global interaction law was used. 
First, the concept of a layered composite inclusion as a representative element in an elastic framework was further extended by adding a third phase, referred to as the interphase or the rigid-amorphous phase. This phase represents the region between crystalline and amorphous domains, having a somewhat ordered structure and a constant thickness. The three-phase composite inclusion model enables to incorporate an internal length scale, which naturally leads to a dependence of mechanical properties on the lamellar thickness, while this effect was not captured in the two-phase model. The comparison of the prediction of the two-phase model with experimental data revealed a good agreement for isotropic material. However, this rigid-amorphous phase is particularly relevant for quantitative modeling of the behavior of oriented semicrystalline structures. To correctly characterize the properties of the rigid-amorphous phase, and its effect on the macroscopic properties, more experimental data of semicrystalline polymers with different morphological configurations are needed.

A key issue in adding quantitative predictive abilities to the micromechanical model for an accurate prediction of the mechanical performance of semicrystalline polymers is the stress-dependence of the microscopic rate of plastic deformation for both crystalline and amorphous domains, which is the mechanism underlying time-dependent, macroscopic failure. The kinetics of macroscopic plastic flow is strongly dependent on the slip kinetics of the individual crystallographic slip systems, accompanied by the yield kinetics of the amorphous domains. In order to achieve an accurate quantitative prediction, the viscoplastic power law relation, previously used in micromechanical modeling, was replaced with an Eyring flow rule, which was shown to be able to better mimic the kinetics of the macroscopic plastic flow. The slip and amorphous yield kinetics were evaluated and characterized using a hybrid numerical/experimental procedure, and the results were validated for uniaxial compression data of HDPE. A double yield phenomenon was observed in the model prediction and was found to originate from the morphological alterations during deformation that cause a change in the deformation mechanism. The predicted texture evolution during deformation, was used to analyze and understand the morphological factors that resulted in a double yield phenomenon in the model.

Experimental data of the yield kinetics of polyethylene at elevated temperatures and various strain rates reveal the contribution of two relaxation processes. Further experimental observations on the stress dependence of the time-tofailure show a piece-wise linear relation in semi-logarithmic plots, with the same absolute slope as that of the yield kinetics, which is an indication of a relation between the short-term and long-term failure kinetics. To quantitatively predict failure under both conditions and for different temperatures, the kinetics at the microscopic scale were characterized using a combined numerical/experimental 
approach, taking into account uniaxial compression and tension experiments of isotropic HDPE, at various temperatures and strain rates. The Eyring flow rule was modified by adding a temperature shift function. A non-Schmid effect was also added to enable the prediction of both tension and compression. The creep behavior of polyethylene was then simulated directly using the multi-scale, micromechanical model, predicting the time-to-failure without any additional fitting parameter. The predicted compressive and tensile yield kinetics and the stress-dependence of the time-to-failure provided a good agreement with experimental data.

Semicrystalline polymeric products are often manufactured from the molten state by common processing techniques, such as injection molding and extrusion. During these processes, the polymer melt experiences shear and elongational flow that induces orientation within the product. The resulting morphology is, therefore, different from that of isotropic polymers, e.g. a stacked lamellar morphology is commonly observed in extruded semicrystalline polymers. For these materials, the mechanical response depends on the direction of loading with respect to the flow direction, and thus their plastic deformation and failure kinetics are anisotropic. The relation between the initially oriented microstructure and the deformation kinetics of oriented polyethylene tapes was studied using the multi-scale micromechanical model. Wide angle X-ray scattering experiments were used to obtain the initial orientation distribution for the model. It was observed that due to the existence of oriented amorphous domains in the drawn samples, the macroscopic deformation kinetics is predominantly governed by the yield kinetics of the amorphous phase, and, therefore, the need of modeling the load angle dependence of the properties of the oriented amorphous domain for an accurate quantitative prediction was discussed. Furthermore, the possibilities for identifying the properties of distinct crystallographic slip systems was investigated.

\subsection{Recommendations}

The main objective of this thesis is adding quantitative predictive ability to the multi-scale micromechanical model for accurate prediction of the mechanical performance of semicrystalline polymers, as characterized by elasto-viscoplastic deformation. However, due to the complex nature of the plastic deformation in these materials, some assumptions and simplifications are made. In this section, some recommendations for future work are listed.

- It has been revealed in the first chapter that the incorporation of the rigidamorphous phase in the composite inclusion model, leads to a dependence 
on the lamellar thickness, i.e. on an internal length scale. This enables having different configurations with the same degree of crystallinity, through different rigid-amorphous layer thicknesses and stiffness. To experimentally obtain different levels of crystallinity and lamellar thickness, samples were subjected to two different crystallization procedures, namely quenching, and isothermal crystallization (annealing) of the quenched samples. The annealing procedure results in an increase in crystallinity but also of the lamellar thickness (see figure 6.1), which is not appropriate for the characterization of the interphase. More experimental data of

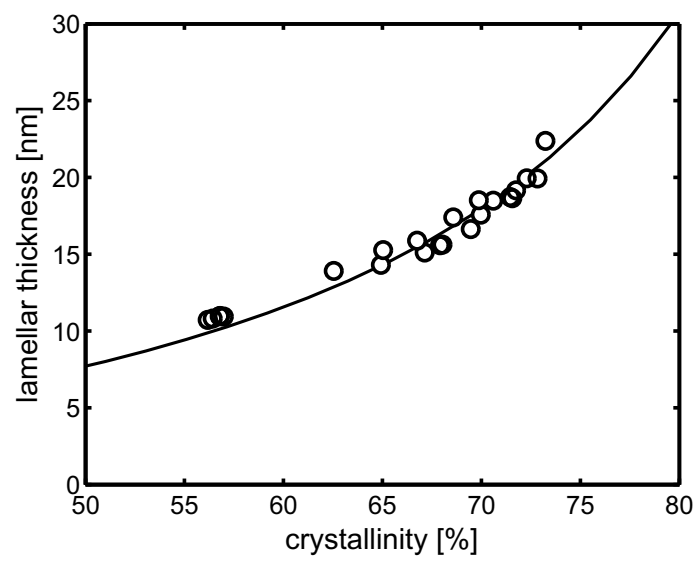

Figure 6.1 Variation of lamellar thickness versus crystallinity of HDPE samples. Symbols give experimental data, and line shows the prediction assuming a constant lamellar spacing, as suggested by Sedighiamiri et al. [1]

semicrystalline polymers with different lamellar thicknesses and a fixed degree of crystallinity are needed to correctly verify the influence of the mechanical properties of the rigid-amorphous phase on the macroscopic properties.

- A dependence of the yield stress on the lamellar thickness is observed in many semicrystalline polymers [2-5]. Crystallographic slip is often considered to occur due to the nucleation of screw dislocations from the lateral surface of the crystalline lamellae. The thicker the lamellar thickness, the more difficult the generation of a dislocation line within the crystals becomes [6], and consequently the yield stress increases. The change in yield stress with increasing crystal thickness is illustrated in figure 6.2. As can be seen, above a lamellar thickness of about $40 \mathrm{~nm}$, the dependence of the yield stress on crystal thickness quickly levels off. This could possibly be an indication of a change of mechanism from nucleation of screw 


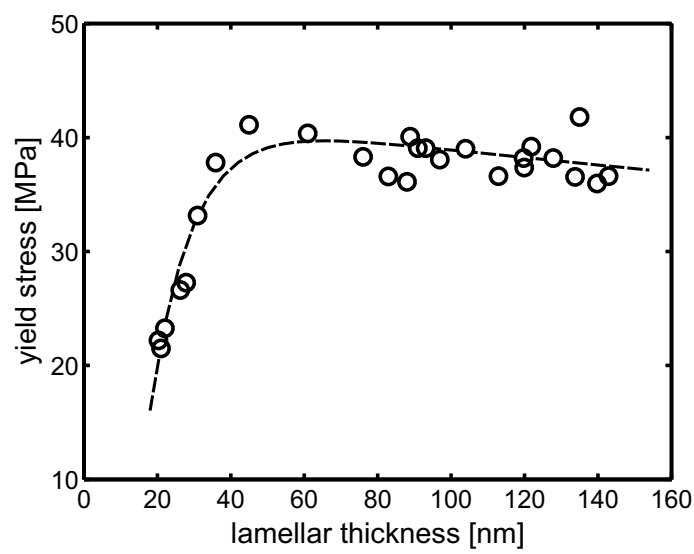

Figure 6.2 Variation of yield stress as a function of lamellar thickness for polyethylene, at a constant strain rate of $10^{-3} \mathrm{~s}^{-1}[5]$. Symbols are experimental data, and line is a guide to the eye.

dislocations to other mechanisms that are independent of the thickness of the lamellae [6]. One of the challenges of adding quantitative abilities to the micromechanical model, would be a description of the lamellar thickness dependence of the crystallographic slip kinetics.

- An important factor for accurate prediction of the stress-strain curves is the strain rate and temperature dependent elastic modulus of the material. A strain rate and temperature dependence of the elastic moduli of the constituent phases is not explicitly described in the model. However, as can seen in figure 6.3, the Young's modulus of polyethylene shows a strong dependency on the temperature and strain rate. A constitutive model, which characterizes the mechanical performance as viscoelastic-viscoplastic deformation, with a temperature dependency of the initial stiffness can provide a more accurate prediction.

- For oriented semicrystalline materials, the mechanical response depends on the direction of loading with respect to the initial orientation direction, and therefore their deformation and failure kinetics are anisotropic. For drawn polyethylene samples, it was observed that due to the presence of oriented amorphous domains in the material, the macroscopic deformation kinetics is mainly governed by the yield kinetics of the oriented amorphous phase. Therefore, for an accurate quantitative prediction, one needs to properly describe the load angle dependence of the properties of the oriented amorphous domain. One possible approach would be modeling a deformation dependent activation volume for the viscous part of the strain 


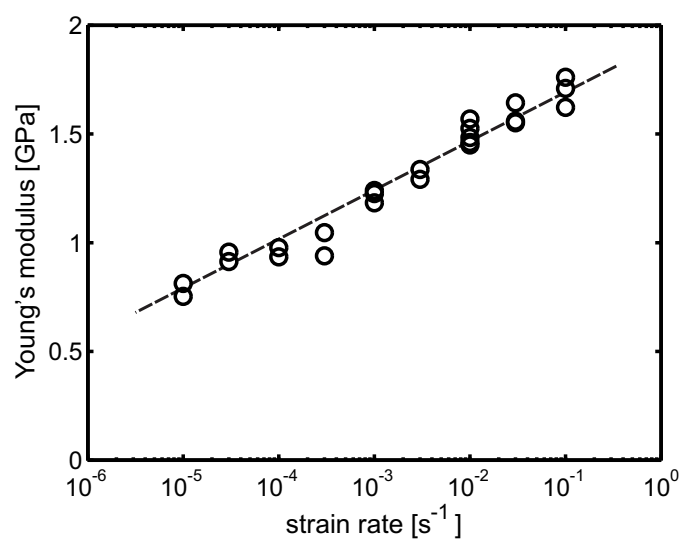

a

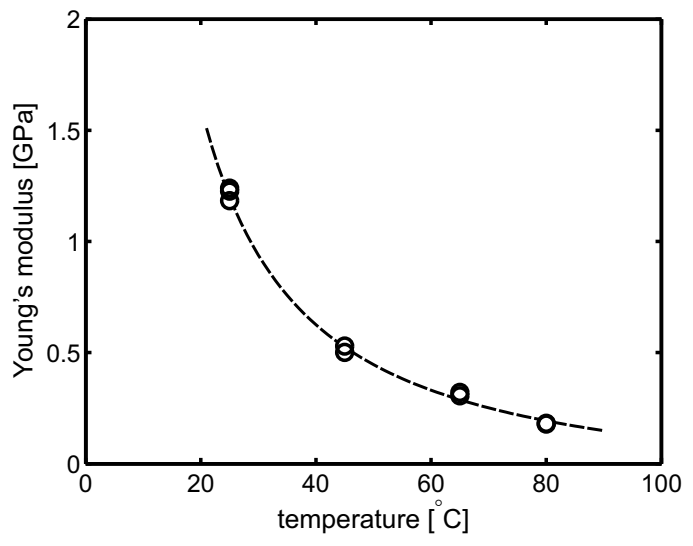

$\mathrm{b}$

Figure 6.3 (a) Strain rate dependency of the elastic modulus of HDPE at $23^{\circ} \mathrm{C}$. (b) Temperature dependency of the elastic modulus of HDPE samples at a constant strain rate of $10^{-3} \mathrm{~s}^{-1}$. Lines are a guide to the eye.

hardening of the amorphous domains, while taking into account a prestretched elastic hardening component, as suggested by Senden et al. [7], within the EGP model.

- There are eight physically distinct slip systems present in the crystalline domain of polyethylene, which comprise six crystallographically different types of slip systems, each possibly having different properties. For an isotropic semicrystalline polymer, the macroscopic plastic flow is governed by the effective contribution of these slip systems, accompanied by the yield kinetics of the amorphous domains. Therefore, it is not possible to completely characterize and distinguish between the effect of different slip systems for an isotropic system, and experimental data of a system with an initially oriented morphology is needed to correctly make a distinction between different types of slip systems and characterize them. However, it was observed that due to the presence of oriented amorphous domains besides the oriented crystalline phase in drawn samples, the deformation kinetics is dominantly influenced by the properties of the oriented amorphous phase. To further characterize the properties of different crystallographic slip systems, experimental data of oriented samples with unoriented amorphous domains are needed. 


\section{References}

[1] A. Sedighiamiri, T.B. van Erp, G.W.M. Peters, L.E. Govaert, and J.A.W. van Dommelen. Micromechanical modeling of the elastic properties of semicrystalline polymers: a threephase approach. Journal of Polymer Science, Part B: Polymer Physics 48:2173-2184, 2010.

[2] N.W.J. Brooks and M. Mukhtar. Temperature and stem length dependence of the yield stress of polyethylene. Polymer 41:1475-1480, 2000.

[3] A. Galeski. Strength and toughness of crystalline polymer systems. Progress in Polymer Science 28:1643-1699, 2003.

[4] R.P.M. Schrauwen, B.A.G.and Janssen, L.E. Govaert, and H.E.H. Meijer. Intrinsic deformation behavior of semicrystalline polymers. Macromolecules 37:6069-6078, 2004.

[5] T. Kazmierczak, A. Galeski, and A.S. Argon. Plastic deformation of polyethylene crystals as a function of crystal thickness and compression rate. Polymer 46:8926-8936, 2005.

[6] A.S. Argon, A. Galeski, and T. Kazmierczak. Rate mechanisms of plasticity in semi-crystalline polyethylene. Polymer 46:11798-11805, 2005.

[7] D.J.A. Senden, J.A.W. van Dommelen, and L.E. Govaert. Strain Hardening and its Relation to Bauschinger Effects in Oriented Polymers. Journal of Polymer Science Part B: Polymer Physics 48:1483-1494, 2010. 



\section{Samenvatting}

Het proces van plastische deformatie in semikristallijne polymeren is gecompliceerd door de aanwezigheid van een verscheidenheid aan mechanismen op verschillende niveaus en is sterk afhankelijk van de kristalliniteit, de onderliggende microstructuur en de evolutie van deze structuur tijdens deformatie. Een macroscopische homogene deformatie gaat vergezeld met diverse deformatiemechanismen in de heterogene microstructuur. Het doel van dit werk is het ontwikkelen van een kwantitatieve relatie tussen de microstructuur en het elastoviscoplastische gedrag van semikristallijne polymeren. Teneinde dit te bereiken wordt een micromechanisch constitutief model gebruikt. In het model is de microstructuur vertegenwoordigd door een verzameling van gelaagde composiete inclusies, elk bestaand uit een kristallijn lamel, dat mechanisch gekoppeld is aan een amorfe laag. De kristallijne fase is gemodelleerd als anisotroop elastisch met plastische deformatie in de vorm van kristallografische slip. De amorfe fase is verondersteld isotroop elastisch te zijn met een snelheidsafhankelijke plastische deformatie en rekversteviging als gevolg van moleculaire oriëntatie. Om het volumegemiddelde gedrag van iedere gelaagde composiete inclusie te relateren aan het gedrag van de verzameling composiete inclusies wordt gebruik gemaakt van een hybride lokaal-globale interactiewet.

Het concept van een gelaagde bicomposiete inclusie als een representatief element is uitgebreid met een derde fase, welke de tussenfase of de star-amorfe fase genoemd wordt. Deze fase vertegenwoordigt het gebied tussen de krijstallijne en amorfe domeinen en heeft een enigszins geordende structuur en een constante dikte. Het opnemen van de tussenfase in het composiete inclusiemodel leidt op een natuurlijke wijze tot een afhankelijkheid van de lameldikte en daarmee van een interne lengteschaal. Deze star-amorfe fase is in het bijzonder relevant voor de kwantitatieve modellering van het gedrag van georiënteerde semikristallijne structuren. Een vergelijking met experimentele data laat een goede macroscopische voorspelling met het tweefasen model zien voor isotroop materiaal.

Een kritische factor voor het toevoegen van kwantitatief voorspellende waarde aan het micromechanische model voor het elastoviscoplastische gedrag van semikristallijne polymeren is de spanningsafhankelijkheid van de snelheid van 
plastische deformatie. Deze slipkinetiek ligt ten grondslag aan het tijdsafhankelijke macroscopische faalgedrag. De kinetiek van macroscopische plastische vloei is sterk afhankelijk van de slipkinetiek van de individuele kristallografische slipsystemen, vergezeld door de vloeikinetiek van de amorfe fase. Om een nauwkeurige kwantitatieve voorspelling te verkrijgen is de viscoplastische machtsfunctie, welke gewoonlijk gebruikt wordt in micromechanische modelleringen, vervangen door een Eyring relatie. De slipkinetiek is daarna opnieuw gekarakteriseerd middels een hybride numeriek/experimentele procedure en de resultaten zijn gevalideerd voor uniaxiale compressie data voor HDPE. Een dubbel vloei effect is waargenomen in de modelvoorspelling en bleek gerelateerd te zijn aan morfologische veranderingen tijdens deformatie waardoor een verandering van deformatiemechanisme optreedt.

Experimentele resultaten voor de vloeikinetiek van polyethyleen voor verschillende temperaturen en reksnelheden laten de bijdrage van twee relaxatieprocessen zien. Verdere experimentele observaties van de snelheidsafhankelijkheid van de tijd-tot-falen laten een lineaire relatie zien op een semilogaritmische schaal, waarbij de helling gelijk is aan die voor de vloeikinetiek. Dit wijst erop dat de kinetiek van falen onder opgelegde reksnelheid en opgelegde spanning sterk gekoppeld zijn. Om falen te voorspellen voor beide condities en voor verschillende temperaturen, zijn de kristallografische kinetiek en de amorfe vloeikinetiek verder verfijnd en is de Eyring relatie uitgebreid met een temperatuursafhankelijkheid. Het kruipgedrag van polyethyleen is daarna direct gesimuleerd met het micromechanische model, waarbij de tijd-tot-falen voorspeld wordt zonder gebruik te maken van een extra parameter. Om het gedrag voor zowel trek als druk te voorspellen, is een niet-Schmid effect toegevoegd aan de constitutieve relatie voor ieder slip systeem.

Polymeren producten, geproduceerd via spuitgieten of extrusie, bezitten een morfologie die verschilt van die van isotrope polymeren door de aanwezigheid van stroming tijdens het proces. Hierdoor zijn hun plastische deformatie en faalgedrag anisotroop. De relatie tussen de initieel georiënteerde microstructuur en de deformatiekinetiek van georiënteerde polyethyleen tapes is onderzocht met het micromechanische model. De initiële oriëntatieverdeling voor het model is gebaseerd op Röntgenverstrooiingsexperimenten (WAXS). Door de aanwezigheid van georiënteerde amorfe gebieden in de getrokken samples wordt het macroscopische plastische gedrag vooral bepaald door de vloeikinetiek van de amorfe fase. De noodzaak voor de modellering van de afhankelijkheid van de eigenschappen van de amorfe fase van de belastingsrichting voor een nauwkeurige kwantitatieve voorspelling wordt besproken. Tenslotte worden de mogelijkheden voor het identificeren van de eigenschappen van verschillende kristallografische slipsystemen onderzocht. 


\section{acknowledgements}

I would like to express my sincere thanks and appreciation to all the people who have helped and supported me throughout my PhD project. Above all, I would like to thank my promoter Prof. Han Meijer for having trust in me and giving me the opportunity to start my PhD without having an interview beforehand. Han, I truly appreciate your continuous supports and helps during these four years.

This thesis would have been hardly possible without the help and guidance of my co-promoters, Dr. Hans van Dommelen and Dr. Leon govaert. I am very grateful to them for the their constant encouragements, positive attitudes during the stressful times, and admirable patience during the numerous fruitful and constructive discussions we have had within these four years. I also would like to thank Prof. Gerrit Peters for his valuable input and interesting discussions during different stages of the project.

In addition to my promoters, I would like to thank Marleen, Yvon and Alice for their helps in many occasions and friendly efforts, which really helped me to feel at home. I wish to thank Leo for his helps in the computer and software problems.

I sincerely thank my committee members, Prof. Charles Tucker, Prof. Issam Doghri, Prof. Theo Tervoort and Prof. Gerrit Peters for their effort in reading my thesis and attending my defense.

My special thanks go to my (former) officemates Jan-Willem, Luigi, Joris, Jana, Tim, Dirk, Dario, Martin and Marc for a pleasant working atmosphere in GEM$\mathrm{Z} 4.22$, and of course for delicious pies and cakes. I am also very thankful to my colleagues and friends in Eindhoven; Zhe, Young Joon, Lambert, Peter, Sam, Murthy, M.K., Tom, Amin, Arash, Zahra, Hamid \& Azar, Javad, Samaneh \& Saeed, Reza, Pouya \& Solmaz, Arash \& Farimah, Ali \& Niloofar, Salman \& Leila, Mohammad \& Flora, Hamed \& Negar, Kamyar \& Pegah, Hamidreza \& Marzieh, Jamilleh \& Peter, Mohammad \& Samaneh, Ali, Mehdi and Parsa for their company and providing me with a very enjoyable social life.

No words can express my gratitude to my parents for their abiding love and caring under any circumstances. Maman, Baba, you have always supported me in every decision I have made. Thank you! I would like to thank my 
brothers, Mobin and Novin, for many wonderful and happy moments we have had together.

Finally, my special gratitude and love goes to my dear wife, Parisa, for her sincere support and enormous love, particularly, in final stressful stage of my PhD. Parisa, I cannot thank you enough for your support, encouragement and tolerating my difficult moments.

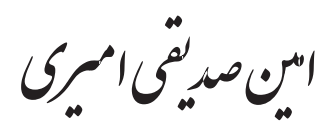

Amin Sedighiamiri

Eindhoven, July 2012. 


\section{Curriculum Vitae}

Amin Sedighiamiri was born on 8th of July 1982 in Babol, Iran. After finishing his high school and a pre-university education at Abu Rayhan in 2000, he entered University of Mazandaran, where he obtained his B.Sc degree in mechanical engineering - solid mechanics in 2005. He continued his education in the same university, and got his M.Sc degree in mechanical engineering - applied design in 2008, on the thesis entitled "Theoretical and numerical elastic-plastic contact analysis of two bodies with rough surfaces". This work was performed under the supervision of dr. M.H. Hojjati, at the Department of Mechanical Engineering, University of Mazandaran. In 2008, he moved to the Netherlands, where he took a position as a PhD student in the Polymer Technology Group, chaired by prof.drir. H.E.H. Meijer, at Eindhoven University of Technology. His $\mathrm{PhD}$ research, which concerns micromechanical modeling of semicrystalline polymers, is a part of a research project entitled "Predicting Catastrophic Failure of Semicrystalline Polymer Products", supported by the Dutch Foundation Program STW (grant number: 07730). During his PhD, he successfully completed three modules of the postgraduate course Register Polymer Science, organized by the National Dutch Research School PTN (Polymeer Technologie Nederland). The completed modules are: module B - Polymer Physics, module C - Polymer Properties, module D\&E - Rheology \& Polymer Processing. 
